University of Louisville

ThinkIR: The University of Louisville's Institutional Repository

Electronic Theses and Dissertations

$12-2011$

\title{
TRAF6 signaling in skeletal muscle atrophy and regeneration.
}

Pradyut K. Paul

University of Louisville

Follow this and additional works at: https://ir.library.louisville.edu/etd

\section{Recommended Citation}

Paul, Pradyut K., "TRAF6 signaling in skeletal muscle atrophy and regeneration." (2011). Electronic Theses and Dissertations. Paper 1101.

https://doi.org/10.18297/etd/1101

This Doctoral Dissertation is brought to you for free and open access by ThinkIR: The University of Louisville's Institutional Repository. It has been accepted for inclusion in Electronic Theses and Dissertations by an authorized administrator of ThinkIR: The University of Louisville's Institutional Repository. This title appears here courtesy of the author, who has retained all other copyrights. For more information, please contact thinkir@louisville.edu. 


\title{
TRAF6 SIGNALING IN SKELETAL MUSCLE ATROPHY AND REGENERATION
}

\author{
By \\ Pradyut K Paul, M.S.

\begin{abstract}
A Dissertation
Submitted to the Faculty of

School of Medicine of the University of Louisville

in Partial Fulfillment of the Requirements

for the Degree of
\end{abstract} \\ Doctor of Philosophy \\ Department of Anatomical Sciences and Neurobiology \\ University of Louisville \\ Louisville, Kentucky
}

December 2011 


\title{
TRAF6 SIGNALING IN SKELETAL MUSCLE ATROPHY AND
} REGENERATION

By

\author{
Pradyut K Paul
}

M.S., Western Kentucky University, 2007

\section{A Dissertation Approved on}

December $1^{\text {st }}, 2011$

By the following Dissertation Committee:

Ashok Kumar, Dissertation Director

Suresh Tyagi

Martha Bickford

Robert Lundy

Robin F. Krimm 
To my parents

Mrs. Nilima Paul and Mr. Prafulla K Paul

for their love and faith in me 


\section{ACKNOWLEDGEMENTS}

The writing of this dissertation has been an amazing journey and a monumental milestone in my academic life. I could not have commenced on this expedition and traveled this far without the passionate and continued support of advisors, colleagues, friends and family.

First and foremost, I would like to thank my advisor Professor Ashok Kumar. It has been an honor to be his first $\mathrm{PhD}$ student. Over the past four years, he has been the major pillar of my professional life at University of Louisville. Through these four years, he has taught me, both consciously and un-consciously, a scientific way of thinking and a style of doing good science. Dr. Kumar has guided me through every step starting from mentoring to teaching techniques, from advising with experiments to job search. It was

his guidance that has paved my way to success through the rapids of this exciting intellectual exploration. He has been the most perceptive and patient mentor. I have always admired him for his motivation and love for science and enthusiasm for his research. It was most certainly contagious and motivational for me and I have learned immensely from him. I deeply appreciate all his contributions of time, ideas, and funding to make my Ph.D. experience productive and stimulating. Dr. Kumar has been and will always be a constant source of inspiration for me. 
I would like to also thank my advisory committee members, Dr. Robin Krimm, Dr. Martha Bickford, Dr. Robert Lundy and Dr. Suresh Tyagi for their time, valued guidance and mentoring throughout these four years.

I would like to personally thank all the members of the Kumar lab who have contributed immensely to my research and personal time during past four years. The group has been a source of friendships as well as good advice and collaboration. I am especially grateful to Drs. Ashwani Mittal, Shephali Bhatnagar and Siva K Panguluri for their unflagging support throughout my graduate research. My most special thanks are due to Saurabh and his family for being part of my cherishing memories in Louisville and for standing by me in most difficult times.

My time at Louisville was made enjoyable in large part due to the many friends and groups that became a part of my life. I am grateful for the time spent with my backpacking and tracking buddies and our memorable trips to many places. I also cherish my friendship with my great friend, intellectual buddy and partner in mischief, Shoeb. His friendship has greatly enriched me during my time in Louisville.

Lastly, I would like to thank my family for all their love and encouragement. For my parents with all my heart who have certainly blessed my life with an environment in which I could freely pursue my dreams and have the courage to surmount higher mountains and for their support in all my pursuits. And most of all for my loving, supportive, encouraging, and patient wife Ruma whose faithful support during the different stages of this Ph.D. is so appreciated. Thank you. 


\section{ABSTRACT \\ TRAF6 SIGNALING IN SKELETAL MUSCLE ATROPHY AND REGENERATION Pradyut K Paul}

\section{December $1^{\text {st }}, 2011$}

Skeletal muscle is the most abundant tissue in our body that provides a structural framework and regulates important biological processes. It is also a primary reservoir of protein. Skeletal muscle maintains its structural and functional integrity by finely balancing the rates of protein synthesis and degradation. Skeletal muscle also has a very well defined regeneration program to cope with muscle injuries. A disruption in any of these delicately balanced intracellular mechanisms of skeletal muscle results in devastating conditions such as atrophies and chronic injuries. Majority of these debilitating conditions in skeletal muscle eventually lead to morbidity and increased mortality and do not have available therapeutic interventions.

The main aim of my research has been focused on understanding the role of an important adapter molecule tumor necrosis factor associated factor 6 (TRAF6) in skeletal muscle wasting and injury-induced regeneration. Using genetic mouse models of TRAF6 muscle-specific knock-out, this study has elucidated the regulatory role of TRAF6 in intracellular signaling pathways in skeletal muscle catabolism. In atrophic conditions, accelerated proteolytic degradation and activation of major catabolic mechanisms of 
skeletal muscle (p38MAPK, c-Jun N-terminal kinase, AMP activated kinase and NF-kB) cause of loss of skeletal muscle protein content and thus lead to reduced muscle fiber size and contractile ability. Myosin heavy chain, a major contractile protein of skeletal muscle is selectively targeted for degradation in response to different atrophic stimuli. In starvation-induced atrophy, endoplasmic reticulum stress and unfolded protein response were also found to be activated in addition to proteolytic mechanisms. Surprisingly, TRAF6 depletion in skeletal muscle of mice repressed activation of all these mediators of skeletal muscle atrophy and consequently, inhibited skeletal muscle atrophy. Taken together, this study has identified TRAF6 as an important regulator of skeletal muscle catabolic mechanisms in disuse and starvation-induced atrophy.

Injury-induced regeneration of skeletal muscle is a highly complex interplay of different signaling networks and effectors. Our results show that TRAF6 activates proinflammatory signaling and promotes inflammation and necrosis in skeletal muscle and its depletion reduces inflammation and accelerates skeletal muscle regeneration. 


\section{TABLE OF CONTENTS}

\section{PAGE}

DEDICATIONS...................................................................................................................iii

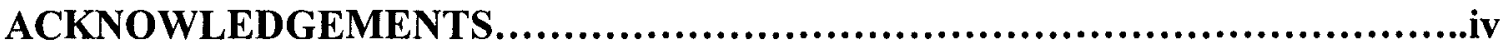

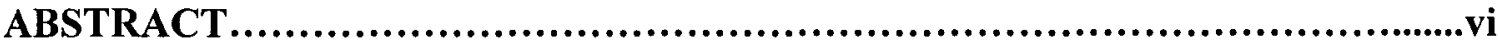

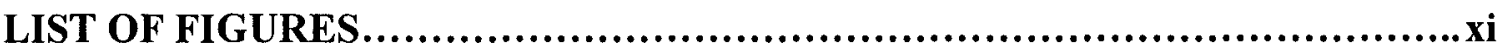

\section{CHAPTERS}

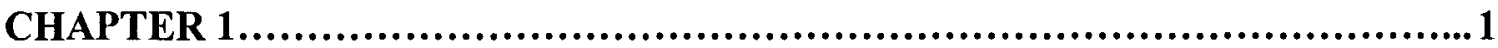

1.1 SKELETAL MUSCLE REMODELING.......................................... 1

1.2 CATABOLIC SIGNALING IN MUSCLE ATROPHY AND

REGENERATION............................................................ 3

1.3 THE TRAF6 SIGNALING AND MUSCLE REMODELING.........................8

1.4 CONTRIBUTION OF TRAF6-SIGNALING IN SKELETAL MUSCLE ATROPHY...........................................................10

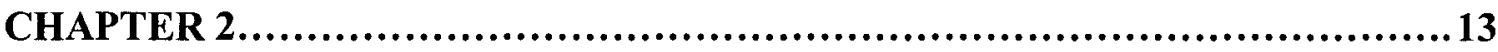

$2.1 \quad$ INTRODUCTION...........................................................13

2.2 MATERIALS AND METHODS...........................................16

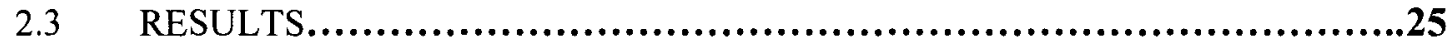

2.3.1 ATROPHIC STIMULI INCREASE EXPRESSION OF TRAF6 IN SKELETAL MUSCLE ...................................................................25

2.3.2 MUSCLE-SPECIFIC DEPLETION OF TRAF6 DOES NOT CAUSE ANY OVERT PHENOTYPE IN MICE.................................................26

2.3.3 DEPLETION OF TRAF6 RESCUES DENERVATION-INDUCED MUSCLE ATROPHY.

2.3.4 INHIBITION OF TARF6 PREVENTS PROTEOLYSIS IN DENERVATED SKELETAL MUSCLE.

2.3.5 TRAF6 IS REQUIRED FOR THE ACTIVATION OF UBIQUITINPROTEASOME AND AUTOPHAGY SYSTEMS IN DENERVATED SKELETAL MUSCLES.

2.3.6 TRAF6 MEDIATES THE ACTIVATION OF JNK, P38 MAPK, AND AMPK IN DENERVATED SKELETAL MUSCLE....

2.3.7 TRAF6 IS INVOLVED IN DENERVATION-INDUCED ACTIVATION OF $N F-\kappa B$ IN SKELETAL MUSCLE. 
2.3.8 DEPLETION OF TRAF6 PREVENTS SKELETAL MUSCLE WASTING IN RESPONSE TO TUMOR GROWTH .........................................34

2.4 CONCLUSION AND DISCUSSION.........................................36

CHAPTER 3..................................................................64

INTRODUCTION.........................................................64

3.2 MATERIALS AND METHODS............................................67

3.3 RESULTS...............................................................71

3.3.1 TRAF6 MEDIATES THE STARVATION-INDUCED FIBER ATROPHY IN MICE ....................................................................71

3.3.2 TRAF6 IS REQUIRED FOR THE ACTIVATION OF UPS AND ALS IN SKELETAL MUSCLE IN RESPONSE TO STARVATION.................. 72

3.3.3 TRAF6 AUGMENTS THE EXPRESSION OF ER STRESS RESPONSE RELATED GENES IN SKELETAL MUSCLE.............................75

3.3.4 TRAF6 IS INVOLVED IN THE INCREASED EXPRESSION OF FNI4 IN SKELETAL MUSCLE UPON STARVATION...............................78

3.3.5 E3 UBIQUITIN LIGASE ACTIVITY OF TRAF6 IS ESSENTIAL FOR STARVATION-INDUCED MUSCLE ATROPHY.........................80

CONCLUSION AND DISCUSSION.......................................83

CHAPTER 4................................................................... 110

INTRODUCTION..................................................... 110

4.2 MATERIALS AND METHODS......................................... 114

4.3 RESULTS.................................................................120

4.3.1 EXPRESSION OF TRAF6 IS INCREASED IN REGENERATING SKELETAL MUSCLE AFTER INJURY.........................................120

4.3.2 ABLATION OF TRAF6 ACCELERATES ADULT SKELETAL MUSCLE REGENERATION IN RESPONSE TO CTX-MEDIATED INJURY........... 121

4.3.3 TRAF6 DEPLETION LEADS TO EARLY RESTORATION OF MUSCLE ARCHITECTURE...........................................................122

4.3.4 DEPLETION OF TRAF6 PROMOTES SATELLITE CELL ACTIVATION IN ADULT SKELETAL MUSCLE IN RESPONSE TO INJURY................123

4.3.5 TRAF6 MEDIATES THE ACTIVATION OF NF- $\kappa B$ IN REGENERATING SKELETAL MUSCLE................................................125

4.3.6 TRAF6-MEDIATED SIGNALING REGULATES THE ACTIVATION OF PRO-INFLAMMATORY AND ANTI-INFLAMMATORY MACROPHAGE PHENOTYPES............................................................ 126

4.3.7 DEPLETION OF TRAF6 EARLY DURING DEVELOPMENT ALSO PROMOTES SKELETAL MUSCLE REGENERATION. 
4.3.8 TRAF6 SIGNALING IN DIFFERENTIATED MYOFIBERS PLAYS A DOMINANT ROLE OVER MYOBLASTS DURING SKELETAL MUSCLE REGENERATION....................................................................129

$4.4 \quad$ CONCLUSION AND DISCUSSION..................................... 130

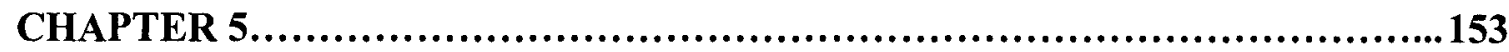

CONCLUSION AND FUTURE WORK

5.1 REVIEW OF DISSERTATION AND SELETAL MUSCLE REMODELING...153

5.2 CONTRIBUTION OF THIS DISSERTATION .................................156

5.3 LIMITATIONS AND FUTURE WORK......................................157

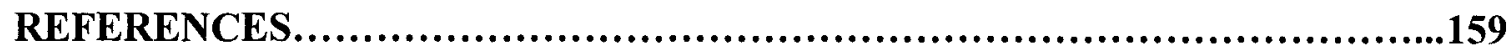

APPENDICES

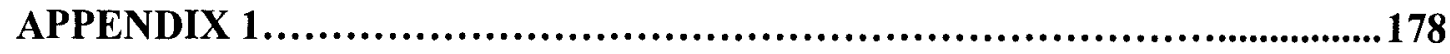

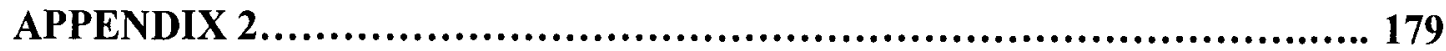

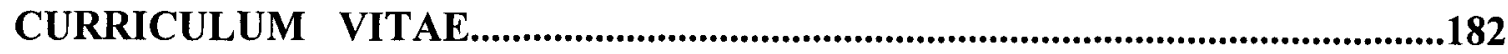




\section{LIST OF FIGURES}

$\begin{array}{ll}\text { FIGURE } & \text { PAGE }\end{array}$

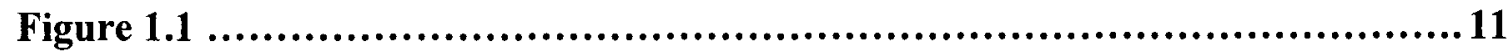

Figure 1.2 ......................................................................... 12

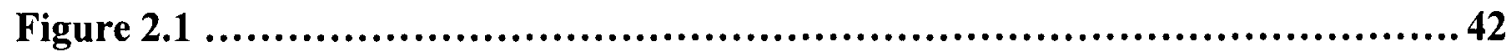

Figure 2.2 .................................................................... 45

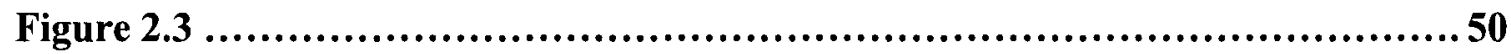

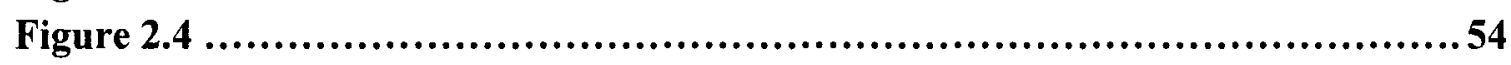

Figure 2.5 ....................................................................55

Figure 2.6 ......................................................................57

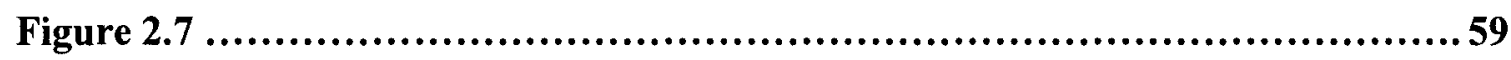

Figure 2.8 ................................................................... 62

Figure 3.1 .................................................................... 89

Figure 3.2 ....................................................................... 94

Figure 3.3 ................................................................... 97

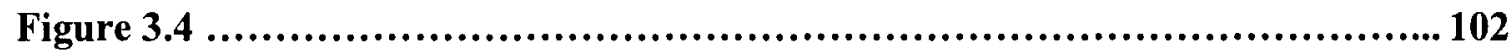

Figure 3.5 .................................................................... 106

Figure 4.1 ....................................................................... 134

Figure 4.2 .................................................................... 135

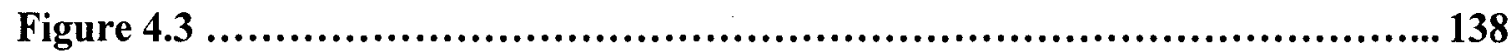

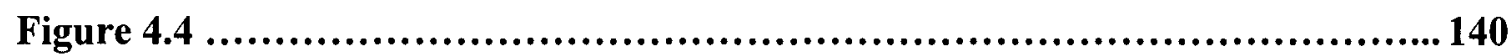

Figure 4.5 .................................................................... 142

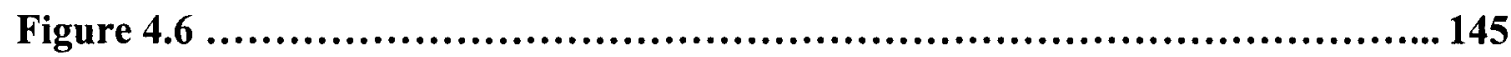

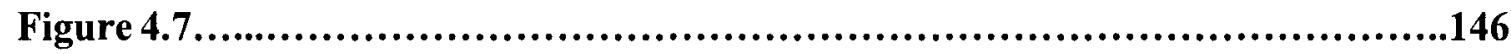

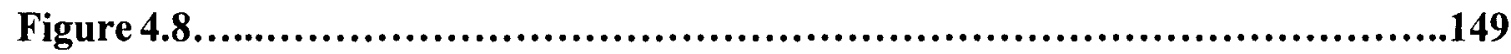




\section{CHAPTER ONE}

\section{INTRODUCTION}

\subsection{Skeletal Muscle Remodeling}

In skeletal muscles, environmental demands and catabolic cues initiate intracellular molecular mechanisms that eventually promote adaptive modifications in muscle architecture and protein composition. Skeletal muscle remodeling occurs in several myopathic abnormalities such as muscle atrophy, muscle injury and regeneration, metabolic disorders, muscle dystrophy and myositis. This study focuses on understanding upstream events in molecular mechanisms that lead to muscle remodeling in muscle atrophy and injury-induced regeneration.

Skeletal muscle atrophy or wasting is an exceedingly debilitating condition that occurs in response to several stress stimuli and chronic diseases. Muscle atrophy is characterized by significant loss of myofibrillar proteins that causes altered myoarchitecture and reduced myofiber size and leads to a reduced muscle tone and compromised contractile ability (1). This devastating complication can occur as a consequence to an astonishing array of catabolic conditions. Stress conditions such as disuse (denervation), immobilization and aging are known to promote skeletal muscle atrophy (2). Muscle atrophy is also a common co-morbidity in a large number of chronic disease states such as cancer, diabetes, chronic heart failure, chronic obstructive 
pulmonary disorder (COPD), renal failure, sepsis and cystic fibrosis (1). An atrophic response is always related to a reduced survival rate, poor functional status and healthrelated quality of life (2). Metabolic alterations such as starvation or nutrient deprivation can also induce atrophy (3). Regardless of the stimulating events, the characteristic features of the atrophy remain the same in all atrophic programs. Loss of muscle protein is a common denominator in almost all atrophic programs. Skeletal muscles are known to be highly plastic and they exhibit little turn-over in normal physiological conditions. However, in atrophic conditions, there is a significant reduction in muscle protein content which is caused by an imbalance between rate of protein synthesis and protein degradation. Since muscles are the primary reservoir of proteins in the body, in certain conditions, it becomes necessary to mobilize muscle proteins into free amino acids. While such an adaptation is required and is beneficial in transient states, it is extremely detrimental in sustained catabolic conditions. Though it is now well established that intracellular pathways of proteolytic degradation are the primary systems of muscle protein loss in atrophic conditions, the upstream mechanisms leading to activation of proteolytic systems are as diverse as their extracellular stimuli. These catabolic signaling mechanisms have therefore, occupied the center stage of investigations aimed to explore skeletal muscle atrophy.

Under several pathophysiological conditions of muscles such as dystrophy, degenerative diseases and severe injury; adult skeletal muscles sustain damage and undergo regeneration. Injury-induced muscle regeneration is an integrated response to muscle injury or disease that involves the degeneration of damaged muscle fibers, inflammation, and satellite cell proliferation and differentiation into new myofibers 
(myogenesis). Skeletal muscle injuries can be classified into two types, acute and chronic. In acute injury such as sports or exercise injuries and toxin-induced injuries, necrotic tissue is removed by a transient infiltration of inflammatory cells followed by repair mediated by resident stem cells. However, in chronic injuries such as in several dystrophies, the causal molecular defects retain the degenerative microenvironment and thus make inflammatory infiltration persistent which further causes degeneration of newly regenerated fibers (4). Despite the diverse types of injury stimuli, all regeneration programs share many common elements such as removal of dead or damaged fibers by inflammatory cells and repair mediated by muscle-resident stem cells known as satellite cells (5).

\subsection{Common Catabolic Signaling Pathways in Muscle Atrophy and Regeneration}

All the major tissues contain several proteolytic pathways that are recruited in a context-dependent manner either to maintain homeostasis or to restore normal physiological conditions in a catabolic state. Similarly, skeletal muscle also contains at least five different proteolytic pathways that include ubiquitin-proteasome system, autophagy-lysosome system, $\mathrm{Ca}^{2+}$-dependent calpains, caspase systems and matrixmetalloproteinases (MMPs). Among these, $\mathrm{Ca}^{2+}$-dependent calpains are believed not to play any significant role in skeletal muscle atrophy (6). Calpains are neither systematically activated in different models of atrophy nor they degrade major contractile proteins (6). Role of caspases (except caspase 3) and MMPs in development of skeletal muscle atrophy is comparatively less explored and poorly documented. On the other hand, there are several compelling evidences that ubiquitin-proteasome (UPS) and autophagy-lysosome systems play a key role in modulating mass of skeletal muscles in 
atrophic conditions $(1,7-18)$. Degradation of proteins by UPS is a two step process. First, the target proteins are tagged with polyubiquitin-chains by covalent attachment and then tagged proteins are degraded by a large, multi-subunit complex known as $26 \mathrm{~S}$ proteasome. Ubiquitin is a highly evolutionarily conserved 76-residue polypeptide; it is conjugated to the target protein substrate via a three-step cascade mechanism. Initially, the ubiquitin-activating enzyme E1 activates ubiquitin in an ATP-dependent reaction to generate a high-energy intermediate called E1-S-ubiquitin. Next in the cascade are ubiquitin-conjugating enzymes (UBCs) or E2 enzymes that transfer the activated ubiquitin moiety from E1, via another intermediate, E2-S-ubiquitin, to the substrate of that family. There are several classes of E3 enzymes and depending on the class, ubiquitin is transferred in a unique way from the E2 enzyme to an active site Cys residue on the E3, which generates a third intermediate, ubiquitin-S-E3, before its transfer to the ligase-bound substrate. A class of E3-ligases that contain RING finger, catalyzes direct transfer of the activated ubiquitin moiety to the E3-bound substrate. E3s catalyze the last step in the conjugation process; they covalently attach ubiquitin to the substrate. The ubiquitin molecule is generally transferred to an $\varepsilon-\mathrm{NH}_{2}$ group of an internal Lys residue in the substrate to generate a covalent isopeptide bond. The choice of Lys-residue is of crucial importance since different E3 ligases can poly-ubiquitinate a substrate through different Lys-residues which can mark target substrates for either activation or degradation. Nevertheless, all E3s synthesize a polyubiquitin chain by successive attachment of ubiquitin moieties to internal Lys residues on the previously conjugated ubiquitin molecule. The poly-Ub tagged substrates are recognized by downstream $26 \mathrm{~S}$ proteasome complex and degraded. However, within UPS, the only specificity is 
introduced by the E3 ubiquitin ligases and therefore E3s play a key role in the ubiquitinmediated proteolytic cascade since they serve as the specific recognition factors of the $\operatorname{system}(10,19-21)$.

The other major pathway for degradation of cellular proteins is autophagylysosomal system (ALS). ALS also contributes significantly in the maintenance of the balance between protein synthesis and protein turnover. The protein turnover that occurs at a subcellular scale is mediated primarily by lysosomes. Lysosomal degradation is executed by three different subtypes of autophagy known as macroautophagy, chaperonemediated autohagy, and microautophagy (22). While microautophagy is not yet well characterized in eukaryotic systems and macroautophagy is primarily involved in degadation of cellular compartments, the chaperone-mediated autophagy (CMA) is found to be a major mechanism that is responsible for degradation of cytosolic proteins (22). In context of skeletal muscle remodeling, both macroautophagy and CMA are found to be involved in regulating degradation of defunct cellular compartments and poly-Ub tagged protein aggregates. Many of the catabolic conditions that cause muscle atrophy, are also known to induce autophagy $(8,14,17,18)$. Upon autophagic induction, formation of a single-membrane vesicle begins in the cytosol, that results in the sequestration of cytoplasmic components and protein aggregates. This single membrane vesicle or "lysosome" is an essential component of ALS and contains hydrolases at low pH that assist in unfolding and degradation of proteins. Target recognition and delivery is carried out by different mechanisms depending on the type of target and cellular conditions (23, 24). In macroautophagy, cytosolic cargo is sequestered inside a de novo formed double membrane vesicle or autophagosome. The autophagosome formation is preceded by 
recruitment of autophagic machinery to poly-Ub tagged protein and protein aggregates. Selective degradation of the aggregates is also mediated by target recognition proteins such as p62 or NBR1, which interact directly with ubiquitin moieties and with LC3, one of the essential autophagy proteins that associate with the autophagosome membrane (25). Although autophagy is extensively investigated in other systems, it is only recently that macroautophagy has been implicated in skeletal muscle remodeling $(13-15,18)$. Along with UPS, ALS constitutes an essential component of cellular quality control system (26). More recent evidence has suggested that UPS and ALS both share a set of attributes such as target recognition and tagging (27). In addition, there are other points of convergence between these two proteolytic systems which indicates that these two pathways might share a common regulator that dictates their coordinated actions in catabolic conditions including those leading to skeletal muscle remodeling.

Several other signaling pathways (such as p38 MAPK, ERK1/2, JNK, PI3K/Akt and AMPK-FoxO) are shown to be involved in skeletal muscle remodeling. An important kinase $\mathrm{p} 38 \mathrm{MAPK}$ is reported to trigger TNF $\alpha$-induced skeletal muscle atrophy and is also known to promote myogenesis $(28,29)$. Extracellular regulated kinase ERK1/2 is shown to be involved in pathogenesis of skeletal muscle wasting (30-32). c-Jun Nterminal kinase (JNK) has also been shown to negatively regulate skeletal muscle mass through insulin signaling and caspases $(28,33-36)$. PI3K/Akt kinase is a positive regulator of muscle mass and causes muscle hypertrophy and is implicated downstream of insulin-like growth factor 1(IGF-1) signaling through regulation of muscle specific E3 ubiquitin-ligase where it blocks transcriptional activation of genes by phosphorylating forkhead transcription factors (FoxO). AMP activated kinase (AMPK) is an upstream 
regulator of FoxO and is shown to augment muscle atrophy and inhibit myoblast differentiation (37-40). Downstream of AMPK, FoxO transcription factors have recently been reported to be involved in development of muscle atrophy by regulating some key components of proteolytic machinery $(13,14,17,41,42)$.

Nuclear factor-kappa B (NF- $\mathrm{B}$ ) has emerged as a central regulator of skeletal muscle remodeling. NF- $\mathrm{NB}$ is not only implicated downstream of several catabolic pathways in skeletal muscles $(30,43-45)$, but has also been found to be induced in a vast majority of skeletal muscle disorders $(7,10,46,47)$. NF- $\kappa \mathrm{B}$ upregulates expression of muscle specific E3 ubiquitin-ligases, interacts with components of ALS, promotes myogenesis and inhibits myoblast differentiation. Taken together, the diverse interactions of NF-kB make it a point of convergence for all the major signaling pathways that regulate skeletal muscle remodeling.

Among the remaining factors that influence skeletal muscle, the most important are cytokines. With some exceptions, majority of cytokines are known to be a negative regulator of skeletal muscle growth, proliferation and differentiation. While TWEAK, TNF $\alpha$ and IL-1 $\beta$ are well known to negatively regulate skeletal muscle mass (48-53), IL4, IL6 and IL10 have been shown to have beneficial effects on skeletal muscle remodeling $(54,55)$. Pro-inflammatory and pro-myogenic cytokines in association with chemokines (CCL2 and CCL5) and NF- $\mathrm{KB}$ play an important role in skeletal muscle injury and regeneration especially when there is a significant interplay between myeloid and myogenic cells that decides impairment or improvement in injury-induced regeneration. 


\subsection{The TRAF6 Signaling and Muscle Remodeling}

TRAF6 belongs to a family of conserved intracellular adaptor proteins. These proteins are involved in transduction of signals from cytosolic domain of tumor necrosis factor-receptor (TNFR)-superfamily, the Epstein-Barr virus protein LMP1, the interleukin-1 receptor (IL-1R) and transforming growth factor- $\beta$ (TGF- $\beta$ ) receptor (56, 57). There are seven mammalian TRAFs (TRAF1-7) identified so far (58-60). All TRAFs comprise a conserved C-terminal domain, the TRAF domain (which further consists of a more divergent $\mathrm{N}$-proximal domain known as TRAF-N and a highly conserved C-proximal sub-domain known as TRAF-C)and an N-terminal zinc-binding RING-domain $(58,59)$. While the TRAF domain is responsible for homo- and heterodimerization of the TRAF proteins and their direct and indirect interactions with associated surface receptors; the $\mathrm{N}$-terminal domain (except TRAF1) has been shown to be crucial for the activation of downstream signaling cascades $(61,62)$. TRAF proteins exhibit a specificity for receptors, heterodimerization partners, adaptor molecules, and downstream signal transducers with which these interact, and this specificity originates from their structural differences (61). Distinct from other TRAF proteins, TRAF2 and TRAF6 have been shown to have E3 ubiquitin ligase activity (63). Further, TRAF2 and TRAF6, in association with dimeric ubiquitin-conjugating enzyme Ubc13/Uev1A, catalyze formation of unique Lys63 linked poly-ubiquitin chain instead of conventional Lys48 ubiquitin chains. Unlike Lys48 polymer, Lys63 polymer has been shown to mediate activation of target proteins instead of degradation $(13,39,64,65)$. TRAF6 is further distinguished by features of auto-ubiquitination which results in activation of more downstream kinases (66) and there are some reported remarkable differences between receptor recognition by TRAF6 and TRAF2 (67). 
Several recent studies have cumulatively suggested that TRAF6 is the most unique of mammalian TRAFs not only due to its gene structure and homology, but also due to its capability to mediate signals stemming from various receptor families (59). Recent findings have shown that TRAF6 mediates and in certain cases is indispensible for signaling pathways downstream of TRANCE-RANK axis $(68,69)$, CD40 signaling $(70,71)$ and IL-1R/TLR axis $(6,67)$. It is now well established that downstream of these receptors, TRAF6 is a central regulator in activation of many signaling pathways including NF-kB, p38-MAPK, phosphatidylinositol 3-kinase (PI3K)/Akt, c-Jun Nterminal kinase (JNK) and AMPK kinase $(10,30,39,72-76)$. In addition, TRAF6 also interacts with p62/SQSTM1-LC3 and Beclin-1; both of these axes are well known to be involved in autophagic degradation of target proteins $(48,77)$. More recently TRAF6 has been reported to interact with cytoplasmic domain of Fn14 receptor and thus might be involved in signaling pathways downstream of Fn14(78). TRAF6 is also involved in activation of unfolded protein response (UPR) and ER stress; a recent report provides the first evidence that TLR-mediated signaling regulates activation of XBP1 (an essential component of IRE1-XBP1 branch of UPR) through TRAF6 $(11,79)$. Despite the identification of a vast array of signaling pathways and effector molecules, which TRAF6 is involved in or interacts with, it has only been studied in very limited contexts. While the pathways regulated by TRAF6 are shown to be involved in catabolic conditions in different systems and settings, its role is extensively explored in immune system and development of osteoblasts and osteoclasts $(30,39,80)$. However, majority of signaling pathways regulated by TRAF6 have been implicated in skeletal muscle atrophy. Taken 
together, molecular interactions and regulatory effects of TRAF6, indicate that TRAF6 may play a pivotal role in regulation of skeletal muscle homeostasis (Figure 1.1, 1.2).

\subsection{Contribution of TRAF6-mediated Pathways in Skeletal Muscle Atrophy}

The role of TRAF6 in skeletal muscle remodeling remains unexplored. Its involvement in molecular mechanisms that play key roles in skeletal muscle atrophy, injury and regeneration led to a hypothesis that TRAF6 might act as a negative regulator of skeletal muscle mass and thus may greatly influence skeletal muscle remodeling. This study is the first one to identify role of TRAF6 in development of catabolic responses in skeletal muscle. Experiments conducted through-out this study aim at exploring the molecular mechanisms regulated by TRAF6 and the downstream effects that lead to muscle atrophy or altered regeneration. Findings of this study will not only be vital to our understanding of the role that TRAF6 plays in muscle wasting and injury-induced regeneration, but may also lead to the identification of potentially new targets that can be utilized for the treatment of these skeletal muscle anomalies. 


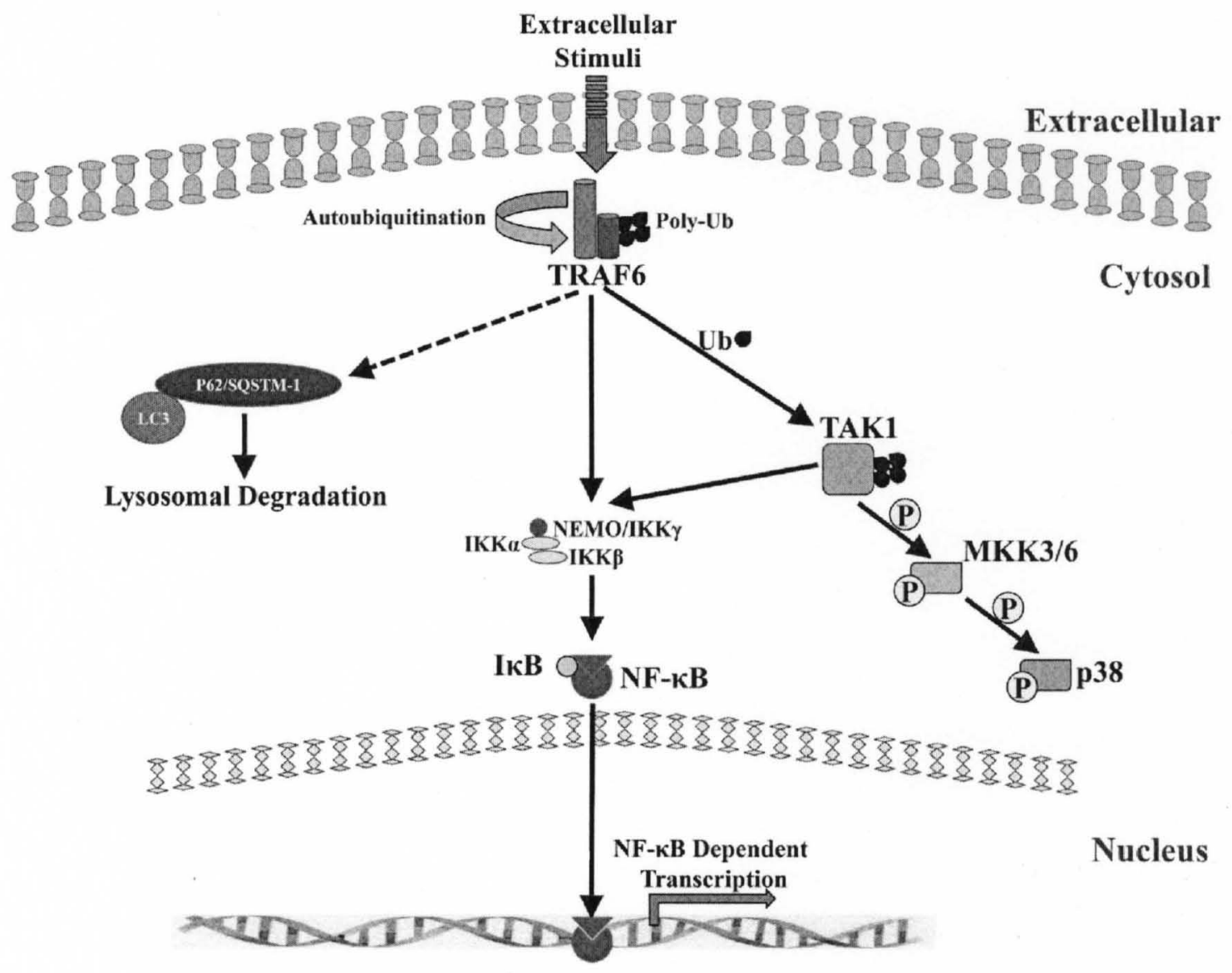

Figure 1.1 


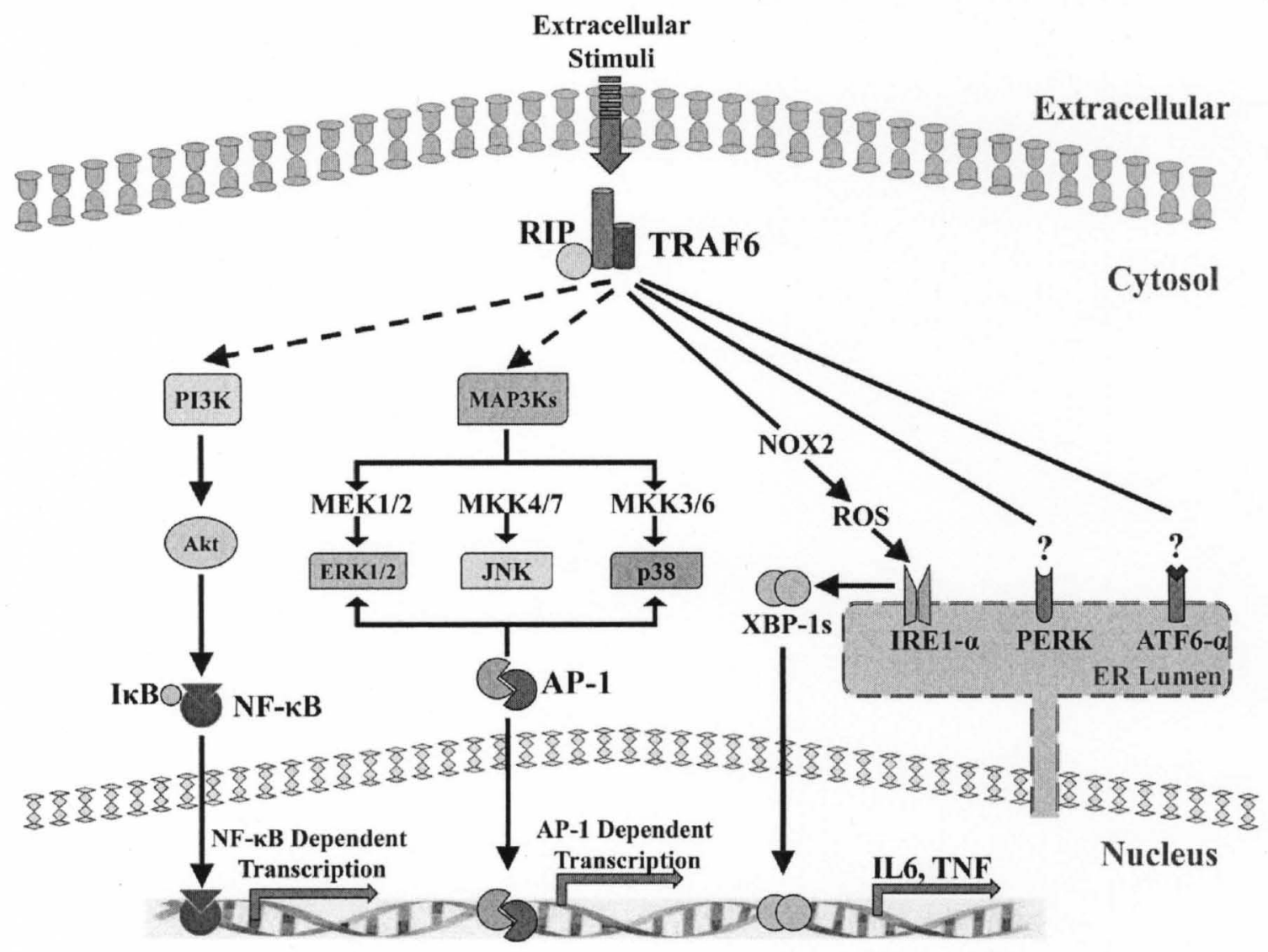

Figure 1.1 and 1.2: TRAF6 Mediated Regulation of Signaling Pathways Relevant to Skeletal Muscle Remodeling 


\section{CHAPTER TWO}

\section{TARGETED ABLATION OF TRAF6 INHIBITS SKELETAL MUSCLE WASTING IN MICE}

\subsection{INTRODUCTION}

Skeletal muscle atrophy or wasting is a complex catabolic response and a devastating complication of stress conditions such as disuse (denervation), immobilization and aging and a large number of chronic disease states such as cancer, diabetes, chronic heart failure, and cystic fibrosis (1). Despite the diversity in stimuli inducing muscle atrophy, an accelerated proteolysis of muscle proteins results from stimulated intracellular proteolytic pathways and leads to a loss of fiber cross-sectional area (CSA), protein content, and functional strength in skeletal muscle $(1,81)$. Recent findings indicate that skeletal muscles respond to different atrophic conditions by activating a complex network of biochemical and transcriptional pathways, leading to the expression of a set of genes termed "atrogenes" $(81,82)$. Many atrogenes are the components of ubiquitin-proteasome system (UPS) that provides a mechanism for selective degradation of regulatory and structural proteins $(11,26,83)$. Two such atrogenes which are also E3 ubiquitin ligases, muscle RING-finger 1 (MuRF1) and muscle atrophy F-box (MAFbx; also called Atrogin-1), have now been identified to be consistently up-regulated in several distinct paradigms of skeletal muscle atrophy in both 
rodents and humans $(9,27)$. Their catabolic role in skeletal muscle has been established by the finding that targeted deletion of MAFbx or MuRF1 rescues atrophy in several conditions $(9,27,84)$. Another major intracellular proteolytic degradation pathways, the autophagy-lysosomal system (ALS) has now been shown to play a crucial role in myofibril proteolysis in skeletal muscle (18). Accumulating evidence further indicates that these two pathways may function in a coordinated manner to augment protein degradation in different atrophy conditions $(13,14,24)$.

Nuclear factor kappa $\mathrm{B}(\mathrm{NF}-\mathrm{kB})$ is a proinflammatory transcription factor that regulates the expression of a large number of genes, including those involved in skeletal muscle proteolysis and fibrosis (85). Increased activation of NF- $\mathrm{KB}$ has been consistently observed in skeletal muscle in distinct models of atrophy (47). One of the important mechanisms by which NF- $\mathrm{kB}$ induces muscle atrophy is through upregulation of $M u R F 1$ $(46,86)$. In addition to NF- $\mathrm{kB}$, several other signaling pathways have also been found to contribute to loss of skeletal muscle mass in catabolic conditions. Activation of p38 MAPK and AMP-activated protein kinase (AMPK) stimulates atrophy by augmenting the expression of $M A F b x$ and $M u R F 1(17,37,87)$, whereas c-Jun N-terminal kinase (JNK) has been implicated in the activation of caspases in atrophying skeletal muscles (36). Moreover, the activation of AMPK and NF- $\mathrm{kB}$ may also stimulate muscle proteolysis through enhancing the activity of autophagy-lysosomal system $(17,38,88,89)$. However, the proximal signaling events leading to the activation of various proteolytic systems in different types of atrophy remain enigmatic. It remains unknown whether the activation of various catabolic pathways is regulated through upstream activation of a 
common signaling network or if they are regulated through independent mechanisms in skeletal muscle in atrophy conditions.

TRAF6 has recently been reported to be crucial for the activation of many signaling pathways including NF- $\mathrm{BB}, \mathrm{MAPK}$, and phosphatidylinositol 3-kinase (PI3K)/Akt in response to cytokines and microbial products $(10,30,39,75,76)$. Of note is the discovery that among all known TRAFs, only TRAF6 interacts with scaffold protein p62/Sequestosome 1 , which is involved in regulation of autophagy and trafficking of proteins to the proteasome $(48,77,90-92)$. More recently, it has been found that TRAF6 promotes the Lys-63-linked ubiquitination of Beclin-1, which is critical for the induction of autophagy, in response to Toll-like receptor 4 signaling (93). Since TRAF6 regulates activation of several signaling cascades and proteolytic systems, which are also activated in atrophying skeletal muscle, it was of obvious interest to indentify whether TRAF6 plays a critical role in regulation of skeletal muscle mass in different catabolic conditions. This study aimed to investigate the physiological functions of TRAF6 in differentiated skeletal muscle and in catabolic conditions. Because conventional TRAF6null mice die perinatally and neonatally because of severe osteoporosis and other defects $(68,94,95)$, for this study, we have generated skeletal muscle-specific TRAF6 knockout mice. Our results show that muscle-specific depletion of TRAF6 preserves skeletal muscle mass, fiber size, and contractile functions in response to denervation. Furthermore, specific inhibition of TRAF6 also inhibits skeletal muscle wasting in a mouse model of cancer cachexia. 


\subsection{MATERIALS AND METHODS}

Mice

A detailed protocol for the generation of floxed TRAF6 (TRAF6f/f) mice has been described previously (94). C57BL6 and MCK-Cre (strain B6.FVB (129S4)-Tg (Ckmm-cre) $5 \mathrm{Khn} / \mathrm{J})$ were obtained from Jackson ImmunoResearch Laboratories, Inc. To investigate the role of TRAF6 in skeletal muscle, we developed a conditional TRAF6 gene inactivation strategy based on the Cre-LoxP system. TRAF6flox/flox $\left(\right.$ TRAF6 $^{\mathrm{f} / \mathrm{f}}$ ) mice were crossed with muscle creatine kinase (MCK)-Cre mice in which Cre-mediated recombination occurs in postmitotic myofibers but not in muscle progenitor cells (96). The muscle-specific TRAF6 knockout thus generated (TRAF6 ${ }^{\text {mko }}$ henceforth) were subjected to various experimental procedures and compared with $\mathrm{TRAF}^{\mathrm{f} / \mathrm{f}}$ mice as control.

\section{Animal Procedures}

Sciatic denervation was performed by anesthetizing the mice with an intraperitoneal injection of Avertin (2,2,2,-tribromoethanol), shaving the left hind quarters, making a $0.5-\mathrm{cm}$ incision $\sim 0.5 \mathrm{~cm}$ proximal to the knee on the flank of the right leg, separating the muscles at the fascia and lifting out the sciatic nerve with a surgical hook or forceps, removing a $2-3-\mathrm{mm}$ piece of sciatic nerve, and finally closing the incision with surgical sutures.

Skeletal muscles from diabetic mice were provided by Dr. Sanjay Srivastava (Diabetes and Obesity Center, University of Louisville, Louisville, KY). Diabetes was induced in 6-wk-old male C57BL6 mice by repeated low-dose STZ $(55 \mathrm{mg} / \mathrm{kg} / \mathrm{d}$ for six 
consecutive days, i.p.) treatment as described previously (97). Mice treated with vehicle only $(0.05 \mathrm{mM}$ sodium citrate, $\mathrm{pH} 4.5)$ served as controls. $1 \mathrm{wk}$ after the last injection of STZ, blood was collected from the tail vein. All the STZ-injected mice had blood glucose $>400 \mathrm{mg} / \mathrm{dL}$. Mice were sacrificed $5 \mathrm{~d}$ after measuring the blood glucose levels. For the cancer cachexia model, LLC cells $\left(2 \times 10^{6}\right.$ cells in $100 \mu$ l saline; American Type Culture Collection) were injected subcutaneously into the flanks of 3-mo-old mice as described previously (46). Mice were weighed daily and sacrificed $14 \mathrm{~d}$ after injection to study muscle atrophy.

For studying NF- $\mathrm{kB}$ reporter gene activity, TA muscle was electroporated with $\mathrm{p}$ NF-kB -Luc (Takara Bio Inc.) and pRL-TK (Promega) as described previously (53). All experimental protocols with mice were approved in advance by the Institutional Animal Care and Use Committee at University of Louisville.

\section{Cell culture}

$\mathrm{C} 2 \mathrm{C} 12$ cells (a myoblastic cell line) were obtained from American Type Culture Collection. These cells were grown in DME containing 10\% FBS. To induce differentiation, the cells were incubated in differentiation medium $(2 \%$ horse serum in

DME) for $96 \mathrm{~h}$ as described previously $(98,99)$. $\mathrm{TRAF}^{+/+}$and $\mathrm{TRAF}^{-/-} \mathrm{MEFs}$ were cultured in DME with 10\% FBS. The cells were plated in 6-well tissue culture plates before treatment with recombinant TWEAK protein (R\&D Systems) for measurement of DNA-binding activity of NF- $\kappa B$ by EMSA. For NF- $\mathrm{KB}$ reporter gene assays, cells plated in 24-well tissue culture plates were transfected with pNF-кB-Luc plasmid (Takara Bio Inc.) using Effectene transfection reagent according to the protocol suggested by the 
manufacturer (QIAGEN). Transfection efficiency was controlled by cotransfection of cells with Renilla luciferase-encoding plasmid pRL-TK (Promega). After treatment with TWEAK, specimens were processed for luciferase expression using a Dual luciferase assay system with reporter lysis buffer per the manufacturer's instructions (Promega). Luciferase measurements were made using a luminometer (Berthold Detection Systems).

\section{Histology and morphometric measurements}

Hind limb muscles (Soleus and TA) of mice were isolated, frozen in isopentane cooled in liquid nitrogen, and sectioned in a microtome cryostat. For the assessment of tissue morphology or visualization of fibrosis, 10- $\mu \mathrm{m}$-thick transverse sections of muscles were stained with $\mathrm{H \& E}$, and staining was visualized (without any imaging medium) at room temperature on a microscope (Eclipse TE 2000-U) using a Plan 10×, NA 0.25 PH1 DL or Plan-Fluor ELWD 20×, NA 0.45 Ph1 DM objective lens, a digital camera (Digital Sight DS-Fi1), and NIS Elements BR 3.00 software (all from Nikon). The images were stored as JPEG files, and image levels were equally adjusted using Photoshop CS2 software (Adobe). Fiber CSA was analyzed in H\&E-stained Soleus or TA muscle sections. For each muscle, the distribution of fiber CSA was calculated by analyzing 200-250 myofibers using NIS Elements BR 3.00 software (Nikon) as described previously (53).

\section{Transmission electron microscopy}

Control and denervated TA muscle isolated from TRAF $6^{\mathrm{f} / \mathrm{f}}$ and TRAF6 ${ }^{\mathrm{mko}}$ mice were fixed in $3 \%$ glutaraldehyde in $0.1 \mathrm{M}$ cacodylate buffer overnight followed by fixing 
in $1 \%$ cacodylate buffered osmium tetroxide. The tissue was dehydrated through a series of graded alcohols, and embedded in LX-112 plastic (Ladd Research Industries). Longitudinal sections $(80 \mathrm{~nm})$ were cut using an ultramicrotome (LKB) and stained with uranium acetate and lead citrate. Samples were analyzed using a transmission electron microscope (CM 12; Philips) operating at $60 \mathrm{kV}$. The pictures were captured at $8,800 \times$ magnification using a 3.2 megapixel digital camera (Sia-7C; Kodak) at room temperature. No imaging medium was used to visualize the pictures, and images were stored as JPEG files. Image levels were equally adjusted using Photoshop CS2 software.

\section{RNA isolation and QRT-PCR}

Isolation of total RNA and QRT-PCR were performed using a method that has been described previously $(98,99)$. In brief, RNA was extracted from homogenized tissues using TRIzol reagent (Invitrogen) and an RNeasy Mini kit (QIAGEN) following manufacturer's protocol. The quantification of mRNA expression was performed using the SYBR Green dye method on a 7300 Sequence Detection system (Applied Biosystems). $1 \mu \mathrm{g}$ of purified RNA was used to synthesize first strand cDNA with a reverse transcription system using an oligo (dT) primer (Applied Biosystems) and Omniscript reverse transcription kit (QIAGEN). The first strand cDNA reaction $(0.5 \mu \mathrm{l})$ was subjected to real-time PCR amplification using gene-specific primers. The sequence of the primers used is described in appendix 1.

Approximately $25 \mu \mathrm{l}$ of reaction volume was used for the real-time PCR assay that consisted of $2 \times(12.5 \mu \mathrm{l})$ Brilliant SYBR Green QPCR Master mix (Applied Biosystems), $400 \mathrm{nM}$ of primers ( $0.5 \mu \mathrm{l}$ each from the stock), $11 \mu \mathrm{l}$ water, and $0.5 \mu \mathrm{l}$ of 
template. The thermal conditions consisted of an initial denaturation at $95^{\circ} \mathrm{C}$ for $10 \mathrm{~min}$ followed by 40 cycles of denaturation at $95^{\circ} \mathrm{C}$ for $15 \mathrm{~s}$, annealing and extension at $60^{\circ} \mathrm{C}$ for $1 \mathrm{~min}$, and, for a final step, a melting curve of $95^{\circ} \mathrm{C}$ for $15 \mathrm{~s}, 60^{\circ} \mathrm{C}$ for $15 \mathrm{~s}$, and $95^{\circ} \mathrm{C}$ for $15 \mathrm{~s}$. All reactions were performed in duplicate to reduce variation. Data normalization was accomplished using the endogenous control ( $\beta$-actin), and the normalized values were subjected to a $2^{-\Delta \Delta \mathrm{Ct}}$ formula to calculate the fold change between the control and experimental groups.

\section{Immunoprecipitation and Western blotting}

Levels of different proteins in skeletal muscle were determined by performing immunoblotting as described previously (100). In brief, tissues were washed with PBS and homogenized in Western blot lysis buffer A (50 mM Tris-Cl, pH 8.0, $200 \mathrm{mM} \mathrm{NaCl}$, $50 \mathrm{mM} \mathrm{NaF}, 1 \mathrm{mM}$ DTT, $1 \mathrm{mM}$ sodium orthovanadate, $0.3 \%$ IGEPAL, and protease inhibitors). Approximately $100 \mu \mathrm{g}$ of protein was resolved on each lane on $10-12 \%$ SDSPAGE, electrotransferred onto nitrocellulose membrane, and probed using anti-TRAF6 (1:1,000; Millipore), anti-TRAF3 (1:1,000; Santa Cruz Biotechnology, Inc.), anti-TRAF4 (1:1,000; Santa Cruz Biotechnology, Inc.), anti-TAB1 (1:1,000; Cell Signaling Technology), anti-phospho p65 (1:500; Cell Signaling Technology), anti-p65 (1:1,000; Santa Cruz Biotechnology, Inc.), anti-phospho-JNK1/2 (1:1,000; Cell Signaling Technology), anti-JNK1/2 (1:1,000; Cell Signaling Technology), anti-phospho p38 (1:200; Cell Signaling Technology), anti-p38 (1:1,000; Cell Signaling Technology), antiphospho-AMPK (1:500; Cell Signaling Technology), anti-AMPK (1:1,000; Cell Signaling Technology), anti-phospho Akt (1:500; Cell Signaling Technology), anti-Akt 
(1:500; Cell Signaling Technology), anti-mTOR (1:1,000; Cell Signaling Technology), anti-phospho-mTOR (1:1,000; Cell Signaling Technology), anti-LC3B (1:1,000; Cell Signaling Technology), anti-Beclin-1 (1:1,000; Cell Signaling Technology), anti-Fn14 (1:1,000; Cell Signaling Technology), MF-20 (1:1,000; Development Studies Hybridoma Bank, University of Iowa), anti-laminin (1:1,000; Sigma-Aldrich), anti-tropomyosin (1:2,000; Sigma-Aldrich), anti-troponin (1:1,000; Sigma-Aldrich), anti-sarcomeric $\square-$ actin (1:1,000; Sigma-Aldrich), anti-nNOS (1:500; Santa Cruz Biotechnology, Inc.), antidystrophin (1:200; Development Studies Hybridoma bank, University of Iowa), antitubulin (1:5,000; Cell Signaling Technology), and anti-MuRF1 (1:1,000; R\&D Systems), then detected by chemiluminescence. The bands were quantified using ImageQuant TL software (GE Healthcare).

To study the auto-ubiquitination of TRAF6, muscle extract ( $400 \mu \mathrm{g}$ protein) was incubated overnight with $1 \mu \mathrm{g}$ anti-TRAF6 antibody (Millipore) in $600 \mu \mathrm{l}$ of lysis buffer followed by addition of protein $\mathrm{A}-$ Sepharose beads and incubation at $4^{\circ} \mathrm{C}$ for additional 2 h. The beads were washed with lysis buffer and finally suspended in Laemmli's sample buffer $(2 \times)$. Proteins were resolved on $10 \%$ SDS-PAGE gel and immunoblotted using anti-ubiquitin (1:1,000; Sigma-Aldrich).

\section{Kinase assays}

For JNK assay, $700 \mu \mathrm{g}$ of muscle extract was immunoprecipitated with antiJNK1 ( $1 \mu \mathrm{g}$ per sample) overnight at $4^{\circ} \mathrm{C}$. This was followed by addition of $30 \mu \mathrm{l}$ of protein A-Sepharose beads. After $2 \mathrm{~h}$, the beads were washed two times with lysis buffer A and two times with kinase assay buffer, then resuspended in $20 \mu \mathrm{l}$ of kinase assay 
mixture containing $50 \mathrm{mM}$ Hepes, $\mathrm{pH} 7.4,20 \mathrm{mM} \mathrm{MgCl} 2,2 \mathrm{mM}$ dithiothreitol, $20 \mu \mathrm{Ci}$ of [y-32P] ATP, $10 \mu \mathrm{M}$ of unlabeled ATP, and $2 \mu \mathrm{g}$ of substrate glutathione S-transferase-cJun (amino acid residues $1-79$ ). After incubation at $37^{\circ} \mathrm{C}$ for $20 \mathrm{~min}$, the reaction was terminated by boiling with $10 \mu \mathrm{l}$ of $4 \times$ Laemmli sample buffer for 3 min. Finally, the protein was resolved on a $10 \%$ polyacrylamide gel, then the gel was dried and the radioactive bands were visualized and quantitated by using a PhosphorImager and ImageQuant TL software (GE Healthcare).

The activity of Akt was assayed similar to JNK1 except that anti-Phospho-Akt conjugated Sepharose beads (Cell Signaling Technology) were used for immunoprecipitation, and GSK-3 fusion protein (Cell Signaling Technology) was used as a substrate in the reaction mixture. The activity of $\mathrm{p} 38$ kinase was measured using a nonradioactive p38 MAP Kinase Assay kit according a protocol suggested by the manufacturer (Cell Signaling Technology). Similarly, AMPK was assayed using a commercially available kit (MBL International).

\section{Electrophoretic Mobility Shift Assay}

$\mathrm{NF}-\kappa \mathrm{B}$ activation in skeletal muscle was analyzed by EMSA as described previously (100), with some modifications. In brief, TA muscles isolated from mice were immediately frozen in liquid nitrogen and suspended at $1 \mathrm{mg}$ of muscle weight per $18 \mu \mathrm{l}$ of low-salt lysis buffer (10 mM Hepes, $\mathrm{pH} 7.9,10 \mathrm{mM} \mathrm{KCl}, 1.5 \mathrm{mM} \mathrm{MgCl} 2,0.1 \mathrm{mM}$ EDTA, $0.1 \mathrm{mM}$ EGTA, $1 \mathrm{mM}$ dithiothreitol, $0.5 \mathrm{mM}$ phenylmethylsulfonyl fluoride, 2.0 $\mu \mathrm{g} / \mathrm{ml}$ leupeptin, $2.0 \mu \mathrm{g} / \mathrm{ml}$ aprotinin, and $0.5 \mathrm{mg} / \mathrm{ml}$ benzamidine) followed by mechanical grinding using a motor and pestle. Cells in the lysis buffer were allowed to 
swell on ice for 10 min followed immediately by three cycles of freeze/thaw lysis. The tubes containing the lysed muscle cells were then vortexed vigorously for $10 \mathrm{~s}$, and the lysate was centrifuged for $30 \mathrm{~s}$ at $14,000 \mathrm{rpm}$. The supernatant (cytoplasmic extract) was removed and saved at $-70^{\circ} \mathrm{C}$ for further biochemical analyses. The nuclear pellet was resuspended in $4 \mu \mathrm{l}$ of ice-cold high-salt nuclear extraction buffer ( $20 \mathrm{mM}$ Hepes, $\mathrm{pH} 7.9$, $420 \mathrm{mM} \mathrm{NaCl}, 1 \mathrm{mM}$ EDTA, $1 \mathrm{mM}$ EGTA, $150 \mathrm{mM} \mathrm{MgCl} 2,25 \%$ glycerol, $1 \mathrm{mM}$ dithiothreitol, $0.5 \mathrm{mM}$ phenylmethylsulfonyl fluoride, $2.0 \mu \mathrm{g} / \mathrm{ml}$ leupeptin, $2.0 \mu \mathrm{g} / \mathrm{ml}$ aprotinin, and $0.5 \mathrm{mg} / \mathrm{ml}$ benzamidine) per milligram of original muscle weight and was incubated on ice for $30 \mathrm{~min}$ with intermittent vortexing. Samples were centrifuged for 5 $\min$ at $4^{\circ} \mathrm{C}$, and the supernatant (nuclear extract) was either used immediately or stored at $-80^{\circ} \mathrm{C}$. The protein content was measured with the method of the Bio-Rad Laboratories protein assay reagent. EMSAs were performed by incubating $20 \mu \mathrm{g}$ of nuclear extract with 16 fmol of the ${ }^{32} \mathrm{P}$ end-labeled NF- $\mathrm{BB}$ consensus oligonucleotides 5'AGTTGAGGGGACTTTCCCAGGC-3' (Promega) for $15 \mathrm{~min}$ at $37^{\circ} \mathrm{C}$. The incubation mixture included 2-3 $\mu \mathrm{g}$ of poly dI-dC in a binding buffer (25 mM Hepes, $\mathrm{pH} 7.9$, $0.5 \mathrm{mM}$ EDTA, $0.5 \mathrm{mM}$ dithiothreitol, $1 \%$ Nonidet P-40, 5\% glycerol, and $50 \mathrm{mM}$ $\mathrm{NaCl}$ ). The DNA-protein complex thus formed was separated from free oligonucleotide on $7.5 \%$ native polyacrylamide gel using buffer containing $50 \mathrm{mM}$ Tris, $200 \mathrm{mM}$ glycine, $\mathrm{pH} 8.5$, and $1 \mathrm{mM}$ EDTA. The gel was dried, and the radioactive bands were visualized and quantitated by a PhosphorImager (GE Healthcare) using ImageQuant TL software. 


\section{Skeletal muscle functional analysis}

The skeletal muscle force production in isometric contraction was performed as described previously $(53,101)$. In brief, soleus muscle from control or denervated hind limb of mice was rapidly excised and placed in Krebs-Ringer solution. The muscle was mounted between a Fort25 force transducer (World Precision Instruments) and a micromanipulator device in a temperature-controlled myobath (World Precision Instruments). The muscle was positioned between platinum wire stimulating electrodes and stimulated to contract isometrically using electrical field stimulation (supramaximal voltage, 1.2-ms pulse duration) from a Grass S88 stimulator (Grass Technologies). In each experiment, muscle length was adjusted to optimize twitch force (optimal length, Lo). The muscle was rested for $15 \mathrm{~min}$ before the tetanic protocol was started. The output of the force transducer was recorded in computer using LABORATORY-TRAX-4 software. To evaluate a potentially different frequency response between groups, tetani were assessed by sequential stimulation at $25,50,75,100,150,200$, and $300 \mathrm{~Hz}$ with $100 \mathrm{~s}$ rest in between.

\section{Statistical analysis}

Results are expressed as mean $\pm \mathrm{SD}$. The Student's $t$ test or analysis of variance was used to compare quantitative data populations with normal distributions and equal variance. A value of $\mathrm{P}<0.05$ was considered statistically significant unless otherwise specified. 


\subsection{RESULTS}

\subsubsection{Atrophic Stimuli Increase Expression of TRAF6 in Skeletal Muscle}

Although TRAF6 is expressed in several cell types, it remains unknown how the expression of TRAF6 is regulated in skeletal muscle cells. Using $\mathrm{C} 2 \mathrm{C} 12$ myoblasts, we first studied how the levels of TRAF6 protein change at different time points after induction of differentiation. As shown in Figure 2.1 A, TRAF6 is expressed in proliferating myoblasts, but its levels are dramatically reduced in differentiated myotubes. Reduced levels of TRAF6 protein appear to be a result of its reduced expression because transcript levels of TRAF6 were also significantly reduced in myotubes compared with myoblasts (Figure 2.1 B). Interestingly, the expression of other TRAFs was not affected during myogenic differentiation (Figure $2.1 \mathrm{~A}$ ). Furthermore, TRAF6 is highly expressed in developing skeletal muscle of young animals but its levels are reduced in adult animals (Figure $2.1 \mathrm{C}$ ). We next determined whether the expression of TRAF6 changes in skeletal muscle in different atrophy conditions. As a model of denervation-induced muscle atrophy, C57BL6 mice were denervated for $4 \mathrm{~d}$ as described previously (53). Lewis lung carcinoma (LLC) cells have been widely used to generate a model for cancer cachexia-induced muscle wasting in mice (46). To determine how expression of TRAF6 is regulated in cachexia, C57BL6 mice were given a single subcutaneous injection of LLC cells (in the left flank), which led to the growth of tumors at the site of injection. After $12 \mathrm{~d}$ of tumor induction, the right hind limb muscles were isolated and analyzed for TRAF6 expression. To induce type I diabetes, C57BL6 mice were treated with chronic intraperitoneal injections of streptozotocin (STZ) for $5 \mathrm{~d}$ as described previously (97). Finally, skeletal muscles of control and challenged mice were 
isolated and processed for studying mRNA and protein levels using quantitative real-time PCR (QRT-PCR) and Western blotting, respectively. As shown in Figure 2.1 D, the mRNA levels of TRAF6 were significantly upregulated in tibial anterior (TA) muscle of mice subjected to denervation, cancer cachexia, or diabetes. Consistent with mRNA levels, the protein levels of TRAF6 were also found to be increased in TA (contains predominantly fast-type fiber) and soleus (contains both slow- and fast-type fibers) in all the three models of atrophy studied (Figure 2.1, E and F).

Because TRAF6 is an E3 ubiquitin ligase that undergoes lysine-63-linked autoubiquitination in response to cytokines and microbial products $(30,39)$, we also investigated whether TRAF6 is ubiquitinated in skeletal muscle under the conditions of atrophy. Protein extracts prepared from control, and denervated TA muscles were immunoprecipitated with TRAF6 antibody followed by Western blotting using ubiquitin antibody. A marked increase in ubiquitinated TRAF6 protein was noticeable in denervated skeletal muscle compared with control muscle (Figure $2.1 \mathrm{G}$ ). These observations suggest that expression and autoubiquitination of TRAF6 are stimulated in skeletal muscle in settings of atrophy.

\subsubsection{Muscle-Specific Depletion of TRAF6 does not Cause any Overt Phenotype in Mice}

The design of targeting construct to generate muscle-specific TRAF6 knockout

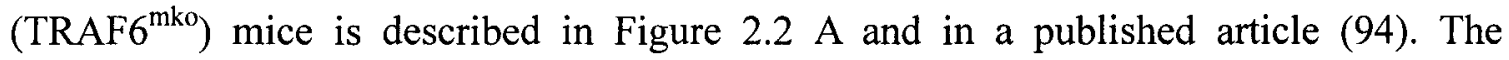
breeding strategy for generation of TRAF $6^{\mathrm{mko}}$ and littermate TRAF6 ${ }^{\mathrm{f} / \mathrm{f}}$ mice is depicted in Figure 2.2 B. Depletion of TRAF6 in skeletal muscle tissues was assessed by 
semiquantitative reverse-transcription PCR using TRAF6 exon 7, internal ribosome entry site (IRES), and glyceraldehyde 3-phosphate dehydrogenase (GAPDH) primers. A significant reduction in TRAF6 transcript levels in skeletal muscle was noticeable in TRAF6 $^{\text {mko }}$ mice, whereas levels of IRES and GAPDH were comparable between C57BL6 (wild-type), TRAF6 ${ }^{\mathrm{t} / \mathrm{f}}$, and TRAF6 ${ }^{\mathrm{mko}}$ mice (Figure $2.2 \mathrm{C}$ ). By performing Western blotting, we also measured protein levels of TRAF6 in skeletal muscle and other tissues of 6-wk-old $\mathrm{TRAF}^{\mathrm{f} / \mathrm{f}}$ and TRAF6 ${ }^{\mathrm{mko}}$ mice. As shown in Figure 2.2 D, the protein levels of TRAF6 were considerably reduced in gastrocnemius (GA) and TA muscle of $\mathrm{TRAF}^{\mathrm{mko}}$ compared with TRAF6 $6^{\mathrm{f} / \mathrm{f}}$ mice. There was no major difference in the levels of TRAF6 in other tissues (e.g., heart, liver, and spleen). Furthermore, the expression of some other TRAFs (e.g., TRAF3 and TRAF4) and an adapter protein TAB1 did not change in skeletal muscle and other tissues of TRAF $6^{\mathrm{mko}}$ and $\mathrm{TRAF}^{\mathrm{f} / \mathrm{f}}$ mice, which indicated depletion of TRAF6 specifically in skeletal muscle of TRAF $6^{\mathrm{mko}}$ mice (Figure $2.2 \mathrm{D})$.

$\mathrm{TRAF}^{\text {mko }}$ pups were viable, born in the expected Mendelian ratio, and indistinguishable from their littermate TRAF $6^{\mathrm{f} / \mathrm{f}}$ mice. There was no significant difference in overall body weight or individual muscle tissue weights between littermate TRAF6 ${ }^{\mathrm{f} / \mathrm{f}}$ and $\mathrm{TRAF}^{\mathrm{mko}}$ mice. At the age of $10 \mathrm{~d}$ (young) and $8 \mathrm{wk}$ (adult), we analyzed muscle tissues of $\mathrm{TRAF}^{\mathrm{f} / \mathrm{f}}$ and $\mathrm{TRAF}^{\mathrm{mko}}$ mice. Hematoxylin and eosin (H\&E) staining of muscle section and histomorphometric analysis showed that the fiber CSA was comparable in TRAF6 $6^{\mathrm{f} / \mathrm{f}}$ and TRAF6 ${ }^{\mathrm{mko}}$ mice (Figure 2.2, E and F). Depletion of TRAF6 in skeletal muscle did not affect the count of fibers per unit area in young or adult mice (Figure 2.2 G). 
Measurement of serum levels of creatine kinase $(\mathrm{CK})$ in $\mathrm{TRAF}^{\mathrm{f} / \mathrm{f}}$ and TRAF6 ${ }^{\mathrm{mko}}$ mice showed no significant difference, which suggests that depletion of TRAF6 does not cause any overt myopathy in mice (Figure $2.2 \mathrm{H}$ ). We also investigated whether TRAF6 regulates the composition of oxidative (type I, slow-type) and glycolytic (type II, fasttype) fibers in skeletal muscle of mice. Staining of soleus muscle section with both antimyosin heavy chain (MyHC) type I and anti-MyHC type II followed by counting of each type of fiber showed that depletion of TRAF6 does not affect fiber composition in skeletal muscle of mice (Figure 2.2 I). Finally, we also found that specific muscle force produced in isometric contractions was similar in $\mathrm{TRAF}^{\mathrm{f} / \mathrm{f}}$ and $\mathrm{TRAF} 6^{\mathrm{mko}}$ mice (Figure $2.2 \mathrm{~J})$. These observations suggest that TRAF6 depletion does not produce any overt phenotype in differentiated muscle of mice.

\subsubsection{Depletion of TRAF6 Rescues Denervation-Induced Muscle Atrophy}

Left hind limb muscles of 3-mo-old $\mathrm{TRAF}^{\mathrm{f} / \mathrm{f}}$ and $\mathrm{TRAF}^{\mathrm{mko}}$ mice were denervated by transecting sciatic nerve, whereas the right hind limb was sham-operated. Gross analyses $14 \mathrm{~d}$ after denervation showed that the loss of GA muscle was considerably reduced in $\mathrm{TRAF}^{\mathrm{mko}}$ mice compared with $\mathrm{TRAF}^{\mathrm{f} / \mathrm{f}}$ mice (Figure $2.3 \mathrm{~A}$ ). We also measured wet weights of different muscles (soleus, TA, and GA) from tendon to tendon. Interestingly, denervation-induced loss of soleus, TA, and GA muscle weight was significantly rescued in TRAF6 ${ }^{\mathrm{mko}}$ compared with $\mathrm{TRAF}^{\mathrm{f} / \mathrm{f}}$ mice (Figure $2.3 \mathrm{~B}$ ). We next performed H\&E staining on TA and soleus muscle sections of control and denervated $\mathrm{TRAF}^{\mathrm{f} / \mathrm{f}}$ and TRAF6 ${ }^{\mathrm{mko}}$ mice and quantified fiber CSA. Interestingly, fiber CSA was significantly preserved in TA (Figs. 2.3, C and D) and soleus (Figs. 2.3, E and F) muscle 
of $\mathrm{TRAF}^{\mathrm{mko}}$ mice compared with littermate $\mathrm{TRAF}^{\mathrm{f} / \mathrm{f}}$ mice upon denervation. Furthermore, force production in isometric contraction was significantly higher in denervated soleus muscle of TRAF6 $^{\text {mko }}$ mice compared with TRAF6 $^{\text {f/f }}$ mice (Figure 2.3 G).

\subsubsection{Inhibition of TARF6 Prevents Proteolysis in Denervated Skeletal Muscle}

Skeletal muscle atrophy can occur due to enhanced proteolysis, reduced protein synthesis, or both $(1,26)$. We investigated the possibility of whether TRAF6 affects myofibril proteolysis or if it represses expression of specific muscle proteins in denervated skeletal muscle. Sham-operated or denervated TA muscles were isolated from $\mathrm{TRAF}^{\mathrm{f} / \mathrm{f}}$ and TRAF6 ${ }^{\mathrm{mko}}$ mice, and muscle extracts made were used to measure the levels of specific muscle proteins by Western blotting. As shown in Figure $2.4 \mathrm{~A}$, the levels of MyHCs were considerably reduced in denervated TA muscle compared with shamoperated TRAF6 $6^{\mathrm{f} / \mathrm{f}}$ mice. Interestingly, the denervation-induced loss of MyHC was rescued in $\mathrm{TRAF}^{\mathrm{mko}}$ mice (Figure 2.4 A). Quantification of band intensities from immunoblots confirmed that the levels of $\mathrm{MyHC}$ were significantly higher in denervated TA muscle of $\mathrm{TRAF}^{\mathrm{mko}}$ mice compared with TRAF $6^{\mathrm{f} / \mathrm{f}}$ mice (Figure $2.4 \mathrm{~B}$ ). Consistent with a previously published study (19), we found that the protein levels of tropomyosin were increased in denervated muscle, though there was no major difference in the levels of tropomyosin in denervated muscle of $\mathrm{TRAF}^{\mathrm{mko}}$ mice compared with $\mathrm{TRAF}^{\mathrm{f} / \mathrm{f}}$ mice (Figure 2.4 A). We also did not find any significant difference in the levels of many other muscle proteins such as troponin, neuronal nitric oxide synthase (nNOS), laminin, $\beta$ dystroglycan, dystrophin, utrophin, and sarcomeric $\alpha$-actin in control and denervated 
skeletal muscle of TRAF6 $6^{\mathrm{f} / \mathrm{f}}$ and TRAF6 ${ }^{\mathrm{mko}}$ (Figure $2.4 \mathrm{~A}$ ). These data are in agreement with previously published findings that muscle wasting involves degradation of only select muscle proteins $(7,53)$.

To further determine whether increased levels of MyHC in denervated skeletal muscle of $\mathrm{TRAF}^{\mathrm{mko}}$ compared with $\mathrm{TRAF}^{\mathrm{f} / \mathrm{f}}$ mice were a result of its reduced degradation or increased expression, we measured mRNA levels by QRT-PCR. Interestingly, there was no significant difference between mRNA levels of MyHC between control and denervated TA muscle of TRAF $6^{\mathrm{f} / \mathrm{f}}$ and TRAF6 ${ }^{\mathrm{mko}}$ mice (Figure 2.4 C). Collectively, these results indicate that the inhibition of TRAF6 prevents the proteolytic degradation of $\mathrm{MyHC}$ without affecting its expression in denervated skeletal muscles.

\subsubsection{TRAF6 is Required for the Activation of Ubiquitin-Proteasome and Autophagy Systems in Denervated Skeletal Muscles}

The ubiquitin-proteasome system is the major pathway that causes the degradation of muscle proteins in various atrophying conditions $(82,83)$. Previous studies have reported enhanced expression of several components of the ubiquitinproteasome system and an increase in the amounts of ubiquitinated proteins in different muscle-wasting conditions, including denervation $(82,83)$. Among several markers of muscle atrophy, two atrogenes, MAFbx/Atrogin-1 and MuRF1, have been found to be highly expressed in atrophying muscles $(9,27)$. To determine whether TRAF6 is involved in expression of MAFbx and MuRF1, we measured their transcript levels in denervated skeletal muscle using QRT-PCR technique. In agreement with published 
reports $(9,27)$, mRNA levels of both MAFbx and MuRF1 were found to be drastically increased in denervated TA muscles compared with sham-injured control muscle (Figure $2.5 \mathrm{~A})$. Interestingly, mRNA levels of MAFbx and MuRF1 were significantly downregulated in denervated muscle of TRAF6 ${ }^{\mathrm{mko}}$ mice compared with $\mathrm{TRAF}^{\mathrm{fff}}$, which suggests that TRAF6 regulates the expression of these two atrogenes in denervated skeletal muscle (Figure $2.5 \mathrm{~A}$ ).

Accumulating evidence suggests that autophagy-lysosome mediated proteolysis also contributes to degradation of muscle protein during atrophy $(13,18)$. We next sought to determine whether TRAF6 affects autophagy in denervated skeletal muscle. To answer this question, we compared the mRNA levels of major autophagy-related genes $L C 3 B$, Beclinl, and Gabarapll, which are reported to be significantly up-regulated in denervated muscles (13). Our results showed that the mRNA levels of $L C 3 B$, Beclinl, and Gabarapll were significantly reduced in denervated skeletal muscle of TRAF6 ${ }^{\text {mko }}$ mice compared with $\mathrm{TRAF}^{\mathrm{fff}}$ mice (Figure $2.5 \mathrm{~B}$ ). In agreement with their mRNA levels, the protein levels of MuRF1, LC3B, and Beclin1 were also found to be reduced in denervated muscle of TRAF $6^{\text {mko }}$ compared with TRAF $6^{\mathrm{f} / \mathrm{f}}$ mice (Figure $2.5 \mathrm{C}$ ).

To further ascertain whether depletion of TRAF6 rescues autophagy, we analyzed control and denervated skeletal muscle of TRAF $6^{\mathrm{t} / \mathrm{f}}$ and TRAF ${ }^{\mathrm{mko}}$ mice by transmission electron microscopy. As shown in Figure 2.5 D, sham-operated skeletal muscle of both $\mathrm{TRAF}^{\mathrm{f} / \mathrm{f}}$ and $\mathrm{TRAF}^{\mathrm{mko}}$ mice contained well-organized myofibrillar structure, and normal subsarcolemmal and intermyofibrillar distribution of mitochondria (indicated by black and blue arrows), with no evidence of autophagosome formation. In contrast, denervated muscle of $\mathrm{TRAF}^{\mathrm{f} / \mathrm{f}}$ mice showed disorganization of mitochondria in 
intermyofibrillar as well as subsarcolemmal space and a drastic increase in autophagic vacuole formation (Figure $2.5 \mathrm{D}$, white arrows) and fusion of mitochondria with autophagosome membrane (red arrows). Interestingly, all these characteristics of muscle atrophy and autophagy were considerably reduced in denervated muscle of TRAF6 ${ }^{\text {mko }}$ mice (Figure 2.5 D). Collectively, these results suggest that TRAF6 is involved in the activation of both ubiquitin-proteasome and autophagy systems in denervated skeletal muscle.

\subsubsection{TRAF6 Mediates the Activation of JNK, P38 MAPK, and AMPK in Denervated Skeletal Muscle}

Recent findings have identified several signaling pathways that regulate skeletal muscle mass in both hypertrophy and atrophy conditions $(26,84)$. Because TRAF6 is a major adaptor protein involved in activation of various cell signaling pathways, we next investigated whether TRAF6 functions through the activation of specific signaling proteins in atrophying skeletal muscle. Control and denervated TA muscle from TRAF6 $6^{\mathrm{fff}}$ and $\mathrm{TRAF}{ }^{\mathrm{mko}}$ mice were isolated, and muscle extracts were analyzed by in vitro kinase assays and Western blotting using antibodies that determine phosphorylated or total levels of specific proteins. Our results showed that denervation augments the kinase activity and phosphorylation of JNK1/2 (Figure 2.6 A) and p38 MAPK (Figure 2.6 B) in skeletal muscle of mice. Importantly, we found that the depletion of TRAF6 was sufficient to block the activation of JNK1/2 (fold change in kinase activity: TRAF6 ${ }^{\mathrm{f} / \mathrm{f}}$, $15.83 \pm 0.41$, vs. TRAF6 ${ }^{\text {mko }}, 12.11 \pm 0.10$ ) and p38 MAPK (fold change in kinase activity: $\mathrm{TRAF}^{\mathrm{f} / \mathrm{f}}, 10.76 \pm 0.74$, vs. TRAF6 ${ }^{\mathrm{mko}}, 7.31 \pm 0.40$ ) in denervated muscles. In 
addition, we also found that the phosphorylation of AMPK as well as its kinase activity were significantly inhibited (fold change in kinase activity: $\operatorname{TRAF}^{\mathrm{t} / \mathrm{f}}, 4.15 \pm 0.24$, vs. $\mathrm{TRAF}^{\mathrm{mko}}, 2.57 \pm 0.39$ ) in denervated skeletal muscle of TRAF6 ${ }^{\mathrm{mko}}$ compared with $\mathrm{TRAF}^{\mathrm{f} / \mathrm{f}}$ mice (Figure $2.6 \mathrm{C}$ ). Because JNK, p38 MAPK, and AMPK are linked with skeletal muscle atrophy $(17,36,87)$, their reduced activation in denervated skeletal muscle of TRAF $6^{\mathrm{mko}}$ suggests that TRAF6 might be mediating skeletal muscle atrophy through the downstream activation of these kinases in response to denervation.

Interestingly, levels of kinase activity and/or phosphorylation of Akt and mTOR, which are involved in anabolic pathways $(26,84)$, were similar in denervated muscle of $\mathrm{TRAF}^{\mathrm{f} / \mathrm{f}}$ and TRAF6 ${ }^{\mathrm{mko}}$ mice (Figure 2.6, D and E). These results suggest that TRAF6 mediates the activation of selective pathways, especially those involved in catabolic mechanisms in denervated muscles.

\subsubsection{TRAF6 is Involved in Denervation-Induced Activation of NF-kB in Skeletal Muscle}

Accumulating evidence suggests that NF-kB is a major transcription factor, the activation of which causes severe muscle wasting in response to diverse stimuli (Li et al., 2008). We investigated whether TRAF6 plays a role in activation of NF- $\mathrm{kB}$ in denervated skeletal muscle. Sham and denervated TA muscle were isolated from $\mathrm{TRAF}^{\mathrm{f} / \mathrm{f}}$ and $\mathrm{TRAF}^{\mathrm{mko}}$ mice, and nuclear extracts were analyzed for NF- $\square \mathrm{B}$ activity by an electrophoretic mobility shift assay (EMSA). The denervation-induced activation of NF$\mathrm{kB}$ in skeletal muscle was inhibited in TRAF6 ${ }^{\text {mko }}$ mice compared with $\mathrm{TRAF}^{\mathrm{f} / \mathrm{f}}$ mice (Figure 2.7, A and B). Furthermore, transcriptional activation of NF-kB measured after 
electroporating an NF- $\mathrm{kB}$ reporter plasmid (Figure $2.7 \mathrm{C}$ ) and the levels of phosphorylation of NF-kB subunit p65 (Figure $2.7 \mathrm{D}$ ) were also significantly inhibited in denervated muscle of $\mathrm{TRAF}^{\mathrm{mko}}{ }^{\mathrm{mice}}$ compared with $\mathrm{TRAF} 6^{\mathrm{f} / \mathrm{f}}$ mice. This suggests that TRAF6 is required for the activation of NF- $\mathrm{kB}$ upon denervation.

It has been recently reported that TNF-like weak inducer of apoptosis (TWEAK) cytokine is a major mediator of denervation-induced skeletal muscle atrophy in mice (Mittal et al., 2010). Normal skeletal muscle expresses TWEAK but not its receptor Fn14. However, in response to denervation, the expression of Fn14 goes up dramatically; this allows for TWEAK activation of NF- $\mathrm{kB}$ (53). We first investigated whether TRAF6 regulates the expression of Fn14 in response to denervation. No major difference was noticed in Fn14 protein levels between TRAF6 ${ }^{\mathrm{f} / \mathrm{f}}$ and TRAF6 ${ }^{\mathrm{mko}}$ mice upon denervation (Figure 2.7 E). We next asked whether TRAF6 is involved in TWEAK-induced activation of NF-kB. To answer this question, we used TRAF6-deficient mouse embryonic fibroblasts (MEFs). Interestingly, TWEAK-induced increases in DNAbinding activity (Figure $2.7 \mathrm{~F}$ ) and transcriptional activation of NF-kB (Figure $2.7 \mathrm{G}$ ) were significantly inhibited in TRAF6 $6^{-1-}$ MEFs compared with $\mathrm{TRAF}^{+/+}$, which indicates that TRAF6 is required for the activation of NF- $\mathrm{KB}$ in response to TWEAK.

\subsubsection{Depletion of TRAF6 Prevents Skeletal Muscle Wasting in Response to Tumor Growth}

Tissue loss is a common consequence in cancer cachexia (102). To understand whether TRAF6 plays any role in cancer cachexia and subsequent muscle loss, $\mathrm{TRAF}^{\mathrm{mko}}$ and $\mathrm{TRAF}^{\mathrm{f} / \mathrm{f}}$ mice were injected with LLC cells in the left flank. Although no 
significant difference was observed in tumor growth in these two mice, skeletal muscle of TRAF $6^{\mathrm{f} / \mathrm{f}}$ mice showed a significant reduction in fiber CSA $14 \mathrm{~d}$ after inoculation with LLC cells (Figure 2.8, A and B). Surprisingly, fiber CSA in LLC-bearing TRAF6 ${ }^{\text {mko }}$ mice was almost completely preserved, which indicates that the TRAF6 mediates the loss of muscle mass in response to tumor growth (Figure $2.8 \mathrm{~B}$ ). Though the molecular basis of cachexia is not yet fully resolved, a majority of factors that induce cachexia involve the activation of NF- $\mathrm{KB}$ at the distal end of their signaling cascade (47). This has been corroborated by the finding that the inhibition of NF- $\mathrm{kB}$ prevents tumor-induced muscle loss in mice (46). We investigated whether TRAF6 functions through the activation of $\mathrm{NF}-\mathrm{KB}$ in this model of cancer cachexia. Our results showed a significant inhibition in DNA-binding activity of NF- $\mathrm{KB}$ in skeletal muscle of LLC-bearing TRAF6 ${ }^{\text {mko }}$ mice compared with TRAF6 $6^{\mathrm{f} / \mathrm{f}}$ mice (Figure $2.8 \mathrm{C}$ ). Furthermore, the expression of $M u R F I$, $L C 3 B$, and Beclinl was blocked in LLCinjected TRAF6 ${ }^{\mathrm{mko}}$ compared with TRAF6 ${ }^{\mathrm{fff}}$ mice; this indicates that, similar to denervation, inhibition of TRAF6 prevents tumorinduced activation of ubiquitin-proteasome and autophagy- lysosomal systems in skeletal muscle (Figure 2.8 D). 


\subsection{CONCLUSION AND DISCUSSION}

The results of the present study indicate a novel role of TRAF6, formerly known as an E3 ubiquitin ligase with involvement in several signaling pathways, in upstream regulation of muscle atrophy. More recently, understanding about the intracellular signaling pathways governing skeletal muscle mass in response to both atrophy and hypertrophy stimuli has taken a quantum leap, though most of the recent investigations were focused on studying the role of various effector kinases and downstream transcription factors $(17,36,37,84,87)$. The initial events that trigger the activation of one or multiple signaling pathways in the conditions of atrophy or hypertrophy remain poorly defined. Our study has identified TRAF6 as a major upstream regulator of skeletal muscle atrophy in response to both physiological (e.g., denervation) and pathological (e.g., cancer cachexia) stimuli. Although it remains unknown what elicits the expression and autoubiquitination of TRAF6 in atrophying muscle, it was of interest to note that proliferating myoblasts express high levels of TRAF6, which is considerably reduced after their differentiation into myotubes. Therefore, it appears that the reduced expression of TRAF6 in differentiated muscle could be a mechanism to prevent the activation of various catabolic pathways under normal conditions. In contrast, elevated levels of TRAF6 in undifferentiated myoblasts or in skeletal muscle of young animals is consistent with published findings that TRAF6 may be required for the proliferation and differentiation of muscle progenitor cells during skeletal muscle development $(103,104)$.

Skeletal muscle atrophy, in different catabolic conditions, involves the downstream activation of the ATP-dependent ubiquitin-proteasome system (83). It has been found that in almost all muscle-wasting conditions, the expression of two muscle- 
specific E3 ubiquitin ligases, $M A F b x$ and $M u R F 1$, which label the target proteins for degradation by $26 \mathrm{~S}$ proteasome, is highly up-regulated $(9,27,82)$. Moreover, a few substrates that $M A F b x$ and $M u R F 1$ target in atrophying skeletal muscle have now been identified (105-107). This study suggests that one of the mechanisms by which TRAF6 induces degradation of muscle protein is through augmenting the expression of both $M A F b x$ and $M u R F I$ in denervated skeletal muscle (Figure 2.5 A). Furthermore, these results demonstrating that the degradation of $\mathrm{MyHC}$ is significantly blocked in denervated skeletal muscle of TRAF ${ }^{\text {mko }}$ mice (Figure 2.4) are in agreement with recent reports that $M u R F 1$ targets thick filament proteins including $\mathrm{MyHC}$ in skeletal muscle $(105,108)$.

Although the exact mechanisms by which TRAF6 augments the expression of $M A F b x$ and $M u R F l$ are not clear, it has been consistently observed that the activity of TRAF6 is stimulated in response to many receptor-mediated events. The N-terminal RING domain of TRAF6 is required for its ability to signal by functioning as an E3 ubiquitin ligase, which catalyzes the synthesis of a polyubiquitin chain linked through Lys-63 (K63) residue in ubiquitin $(10,109)$. This autoubiquitination of TRAF6 serves as a scaffold to recruit molecules required for the activation of kinase complexes such as transforming growth factor $\beta$-activated kinase 1 (TAK1) and IкB kinase $(21,39)$. The TAK1-TAB2 (or TAB3) complex that is activated potentially through TRAF6-dependent ubiquitination can phosphorylate IKK $\beta$ at Ser-177 and Ser-181 in the activation loop, leading to the activation of IKK and subsequently NF- $\mathrm{KB}$ (110). The activated TAK1 complex can also phosphorylate members of the MKK family, leading to the activation of JNK and p38 kinase $(20,22,66)$. Interestingly, recent studies suggest that the activation 
of NF- $\mathrm{kB}$ in skeletal muscle up-regulates the expression of $M u R F$ lin response to a variety of catabolic stimuli, including denervation and tumor growth $(46,47,53,86)$. Furthermore, there are also published studies suggesting that the p38 MAPK augments the expression of $M A F b x$ in response to inflammatory cytokines and bacterial products $(24,87)$. Because depletion of TRAF6 in skeletal muscle blocked the activation of both NF-kB and p38 MAPK in denervated skeletal muscle (Figs. 2.6 B and 2.7), it is likely that TRAF6 augments the expression of $M u R F 1$ and $M A F b x$ through the activation of NF- $\mathrm{kB}$ and p38 MAPK, respectively, by stimulating the activity of TAK1 signalosome. The present study also provides novel evidence that the TWEAK-Fn14 dyad, a major regulator of denervation-induced skeletal muscle atrophy (53), stimulates NF- $\mathrm{kB}$ activation through the recruitment of TRAF6 (Figure 2.7, F and G).

In addition to the ubiquitin-proteasome system, the autophagy-lysosomal pathway has also been implicated in myofibril degradation in various atrophying conditions $(13,18)$. Though it was initially considered as an important mechanism for removal of ubiquitinated protein aggregates and cytoplasmic organelles under the conditions of stress, recent evidence indicates that the activation of this pathway may also be a protective mechanism for muscle fibers in the conditions of atrophy $(13,15,18)$. Mitochondria are one of the most important organelles that undergo alterations in their content, shape, and function in conditions of muscle wasting $(99,111)$. More recently, it has been found that the mitochondrial fission is a prerequisite for skeletal muscle atrophy in response to starvation or after overexpressionof FoxO3, and that the autophagylysosomal system is the major mechanism for the removal of disintegrating mitochondria in these conditions (17). The removal of leaky mitochondria, releasing pro-apoptotic 
factors such as cytochrome $c$ and apoptosis-inducing factor, may protect cells by preventing activation of apoptosis $(17,23,24)$. The protective role of autophagy in skeletal muscle in catabolic conditions has also been highlighted by a recent study demonstrating that muscle-specific depletion of $\mathrm{Atg} 7$, an important component of the autophagy-lysosomal system, led to more severe myopathy in conditions of denervation (15). This suggests that physiological autophagy may be required for muscle homeostasis, whereas its overstimulation in atrophying muscle may contribute to muscle proteolysis (18). Intriguingly, our findings revealed that the inhibition of TRAF6 dramatically reduces mitochondrial disorganization and autophagosome formation (Figure $2.5 \mathrm{D}$ ), as well as the activation of AMPK (Figure 2.6 C), which is known to induce the expression of several autophagy-related genes (e.g., LC3B and Bnip3), and the E3 ligases $M u R F 1$ and $M A F b x$ through the activation of $\mathrm{FoxO} 3$ transcription factor in skeletal muscle $(13,14,17)$. Although mitochondrial fission has been suggested as an initial event to activate the autophagy pathway in skeletal muscle (17), it has been recently demonstrated that TRAF6 causes the Lys-63-linked ubiquitination of Beclin1 (the mammalian homologue of yeast Atg6), which is essential for autophagosome formation in response to Toll-like receptor 4 (TLR4) signaling (93). Whether TRAF6 stimulates autophagosome formation through augmenting mitochondrial fission or if it directly regulates the expression and activity of the components of autophagy-lysosomal pathway is an area of research for future investigation.

We further investigated the contribution of TRAF6 toward muscle loss in cancer cachexia. Earlier studies have underlined the importance of inflammatory cytokines and tumor-derived factors as mediators of muscle loss in both animal models and advanced 
stages of cancer patients $(102,112,113)$. Using transgenic mice expressing a constitutively active mutant of IKK $\beta$, Cai et al. (2004) have previously demonstrated that the stimulation of the NF- $\kappa \mathrm{B}$ pathway is sufficient to cause severe muscle loss in mice. Furthermore, their study also showed that muscle-specific overexpression of a superrepressor mutant of $\mathrm{I} \kappa \mathrm{B} \alpha$ (inhibitor of $\mathrm{NF}-\kappa \mathrm{B}$ ) significantly rescued muscle loss in response to tumor growth in mice (46). The present study provides convincing evidence that TRAF6 is an upstream regulator of LLC-induced muscle loss, NF- $\mathrm{BB}$ activation, and MuRF1 expression (Figure 2.8). Interestingly, although NF- $\mathrm{BB}$ was found to regulate only the expression of MuRFl(46), our results indicate that TRAF6 also regulates the expression of autophagy-related genes (e.g., $L C 3 B$ and Beclinl) in skeletal muscle of LLC-bearing animals (Figure $2.8 \mathrm{D}$ ). Furthermore, the almost complete inhibition of LLC-induced expression of MuRF1, LC3B, and Beclin1 in skeletal muscle of TRAF6 ${ }^{\text {mko }}$ mice compared with control mice is consistent with major amelioration in muscle atrophy in TRAF $6^{\text {mko }}$ mice (Figure 2.8, A and B).

The results of the present study suggest that though a significant inhibition in muscle atrophy was observed in models of both denervation and cancer cachexia, depletion of TRAF6 resulted in more drastic improvement in fiber CSA in skeletal muscle of LLC-bearing mice. A better rescuing effect in the cancer cachexia model compared with denervation could be attributed to the fact that cancer cachexia involves systemic inflammation, and proinflammatory cytokines are some of the most important mediators of muscle wasting in a cancer-bearing host $(102,112,113)$. Interestingly, several cytokines and tumor-derived factors require TRAF6 for the downstream activation of various cell signaling pathways such as NF- $\mathrm{KB}$ and MAPK, which were also 
activated in atrophying skeletal muscle $(30,103,104)$. In contrast, denervation-induced muscle atrophy does not involve any systemic inflammation, though it was recently demonstrated that the TWEAK-Fn14 dyad is one of the important mediators of muscle loss under conditions of denervation $(1,53)$. Although a significant amelioration in muscle atrophy was observed, the denervation-induced muscle loss was also not completely blunted in TWEAK-KO mice (53). Therefore, it is possible that muscle atrophy in response to denervation also involves some other uncharacterized factors that function independent of TRAF6. Nevertheless, the present study provides strong evidence that TRAF6 is central regulator of major proteolytic pathways in different types of atrophy.

In summary, the broad benefits of TRAF6 blockade in skeletal muscle unveil novel and exciting possibilities for therapeutic approaches for the treatment of muscle wasting diseases in humans. 

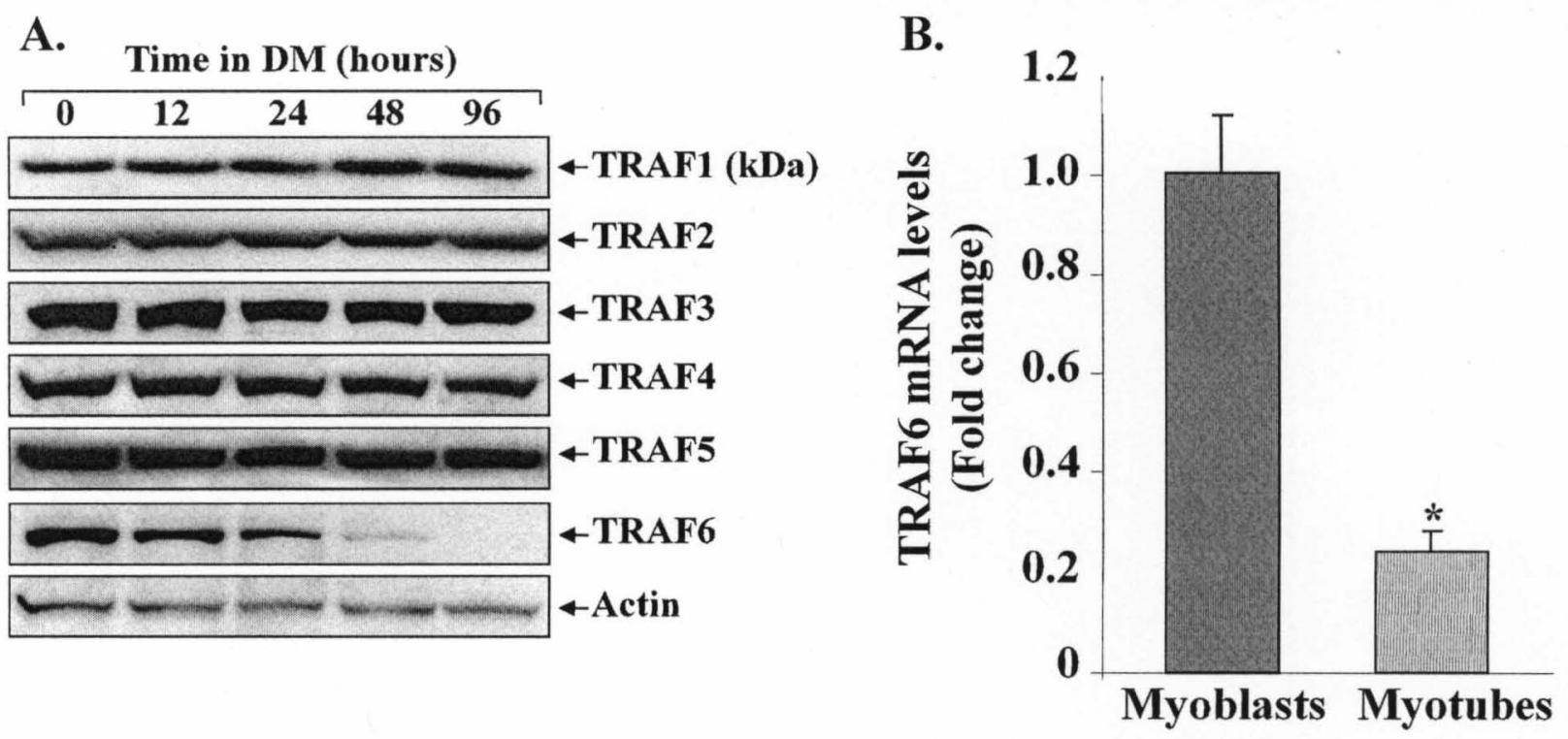

C.

Age of mice (weeks)

\begin{tabular}{|c|c|c|c|c|c|}
\hline 1 & 2 & 3 & 5 & 8 & 12 \\
\hline & & & $=$ & $=$ & $=$ \\
\hline
\end{tabular}


D.
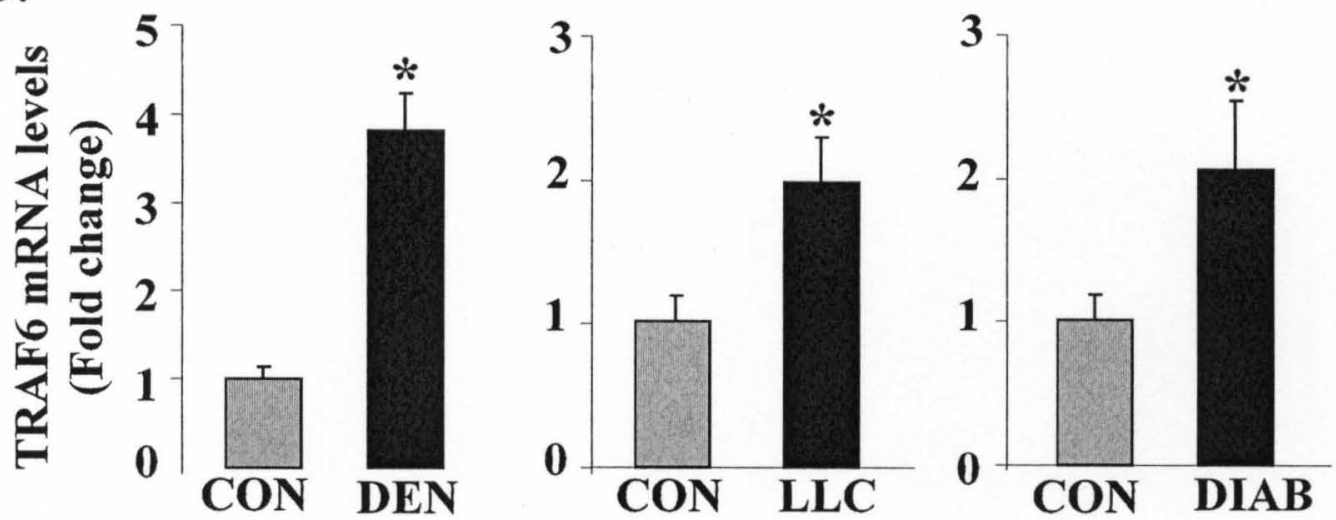

E.

TA muscle

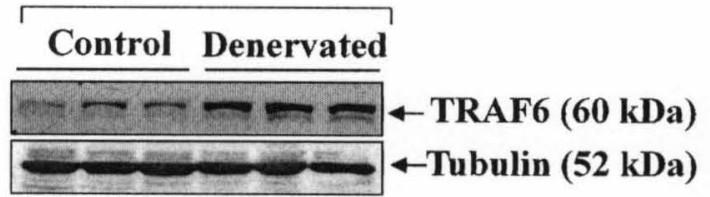

F.

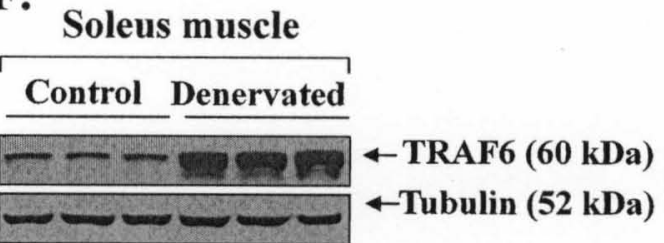

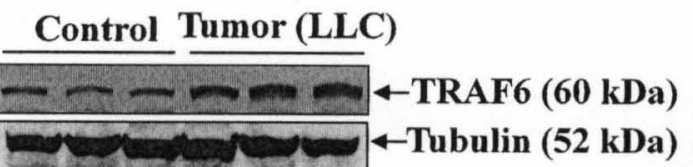

Control Tumor (LLC)

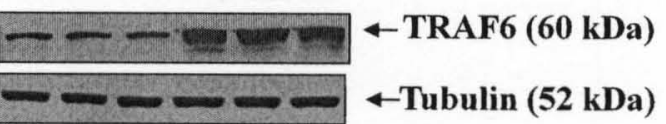

Control Diabetic
$-\square-$ TRAF6 (60 kDa)
$-\square-T u b u l i n(52 \mathrm{kDa})$

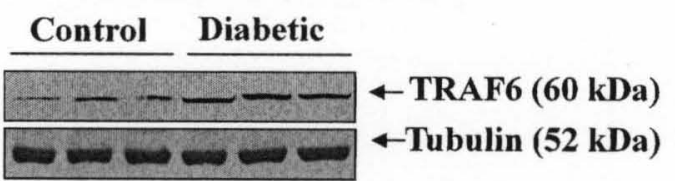




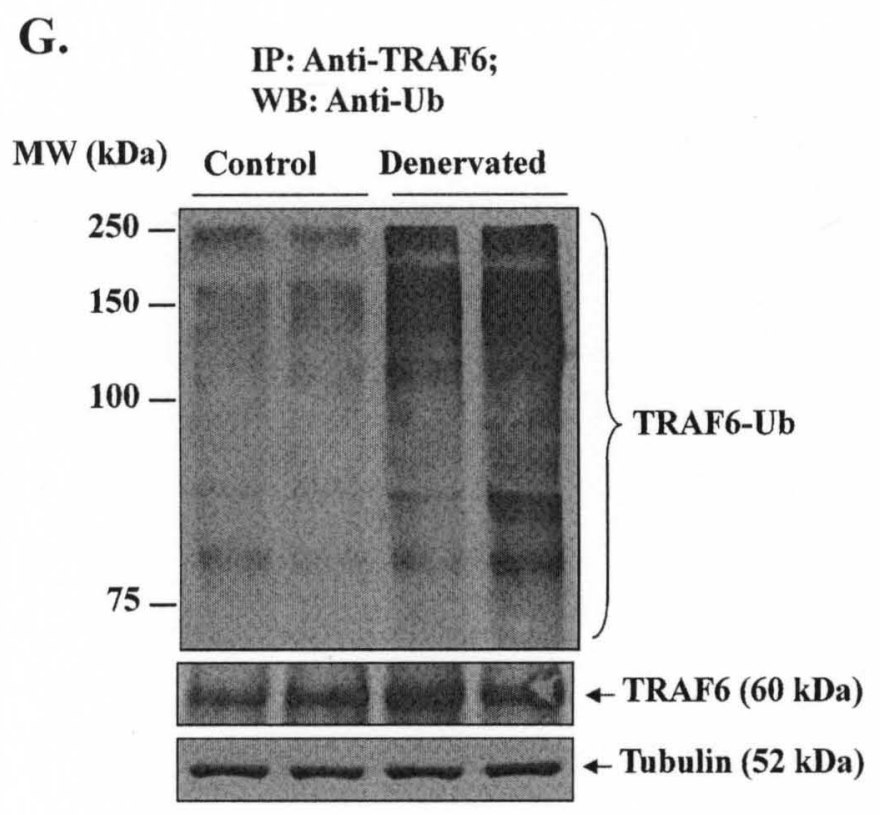

Figure 2.1: Expression of TRAF6 is increased in atrophying skeletal muscle. (A) Western blot analysis of protein levels of TRAF-family member proteins at different time points after induction of differentiation in cultured $\mathrm{C} 2 \mathrm{C} 12$ myoblasts. (B) Change in transcript levels of TRAF6 in response to differentiation of $\mathrm{C} 2 \mathrm{C} 12$ myoblasts into myotubes. (C) Western blot analyses demonstrating age-dependent change in protein levels of TRAF6 in mice. C57BL6 mice were subjected to either the conditions of denervation (sciatic nerve transection), tumor growth (LLC), or diabetes (STZ injection), and TA muscle was isolated and used for biochemical analyses. (D) Fold change in mRNA levels of TRAF6 in TA muscle of challenged mice compared with control mice. (E and F) Western blot analyses of protein levels of TRAF6 in TA muscle (E) and soleus muscle (F) of control and challenged mice. Black lines indicate that intervening lanes have been spliced out. (G) Control and denervated TA muscle extracts were immunoprecipitated with TRAF6 antibody followed by Western blotting using ubiquitin (Ub) antibody (top). Western blotting using anti-TRAF6 after stripping the membrane (middle) and anti-tubulin (bottom). Error bars indicate SD. *, P $<0.01$ (values significantly different from controls). CON, control; DEN, denervated; DIAB, diabetic. 
A.

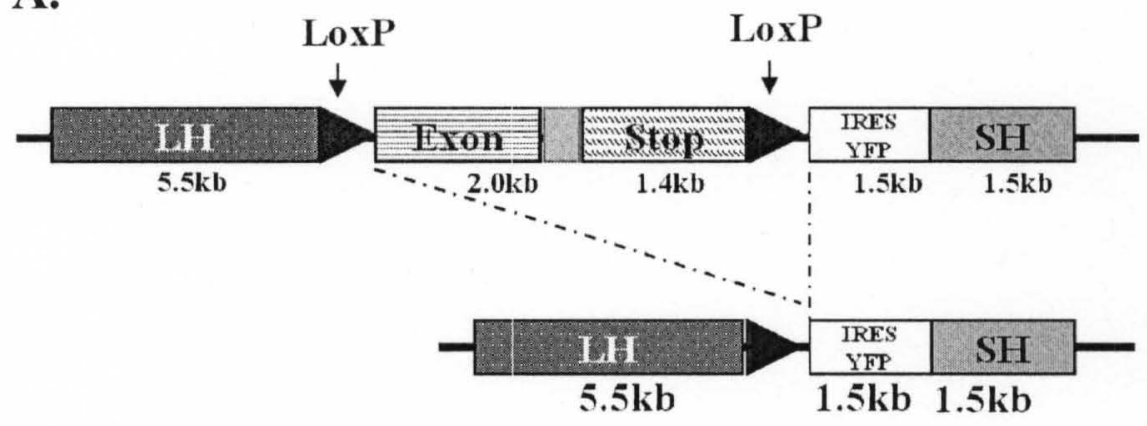

B.

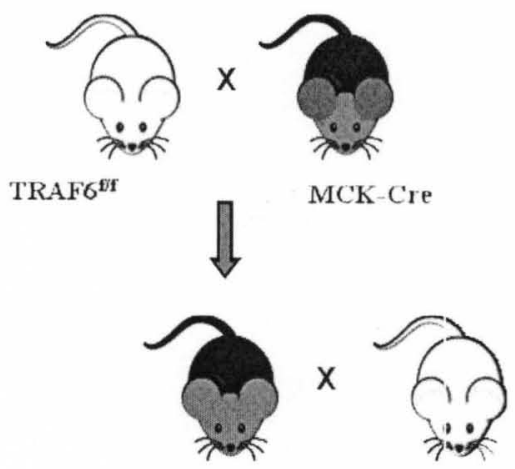

TRAFG ${ }^{\mathrm{f} w t}$; MCK-Cre

TRAFG' (HET)

$\sqrt{2}$

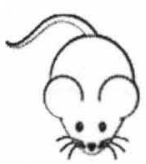

TRAF6 ${ }^{\mathrm{fif}}$ (CONTROL)

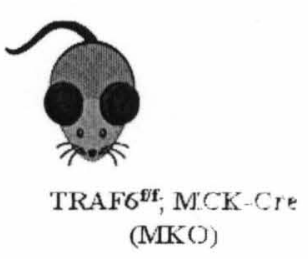



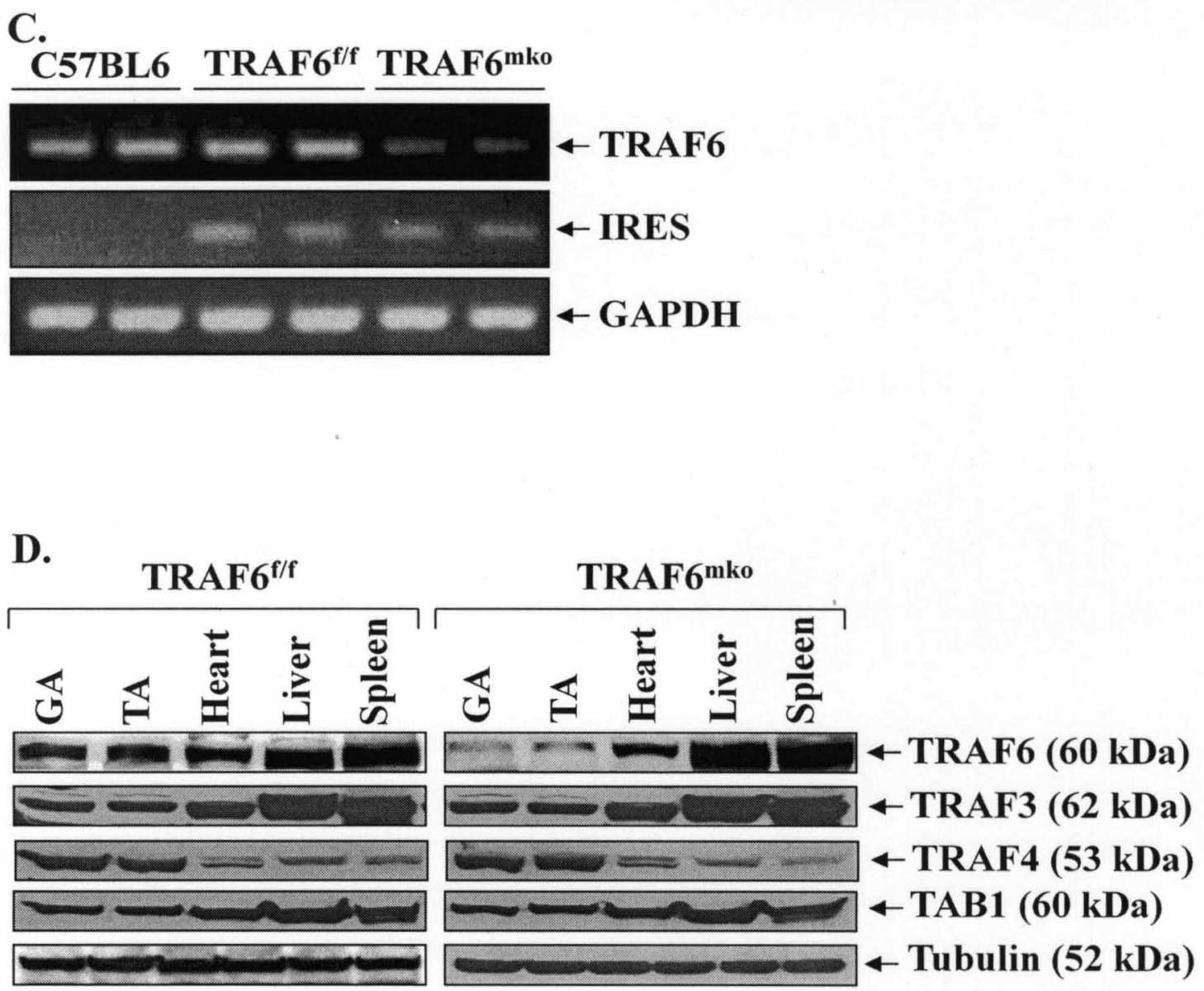

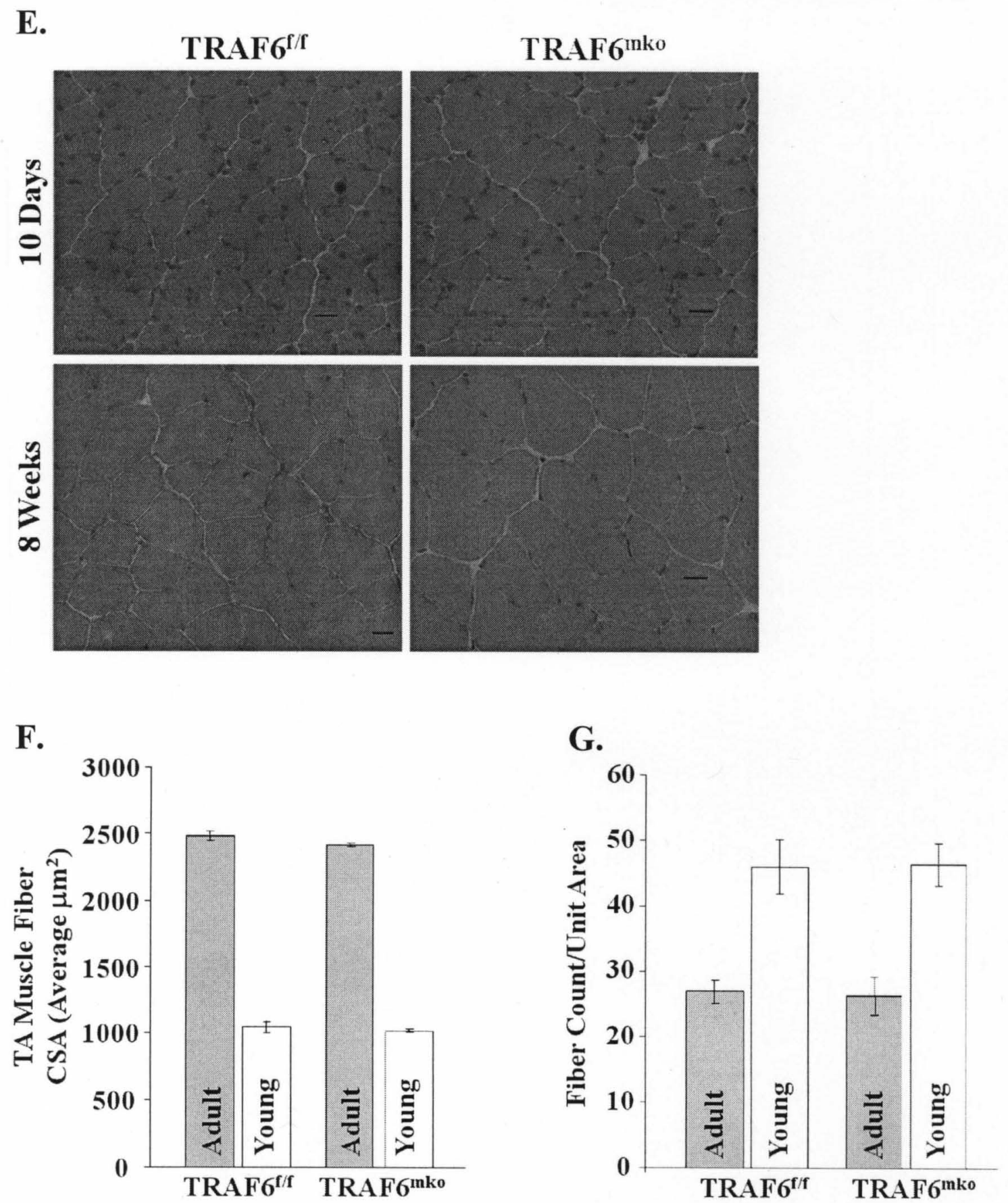
H.

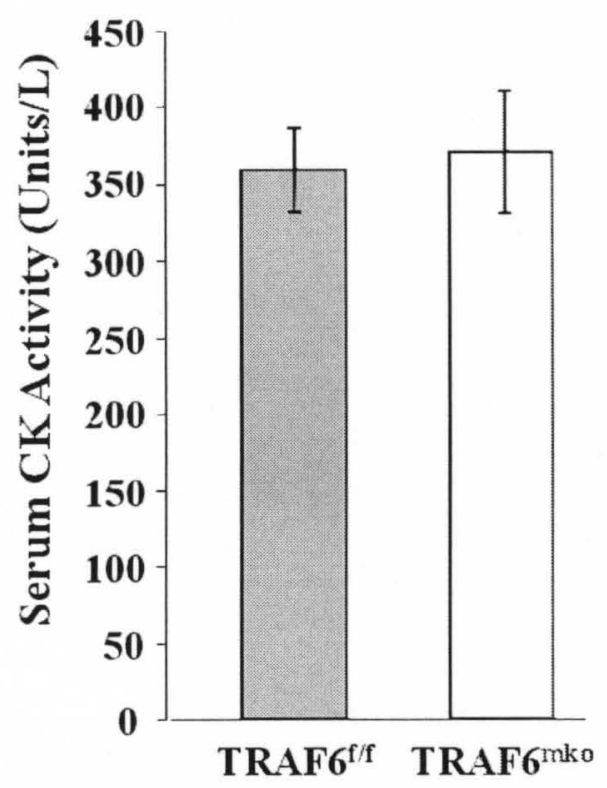

J.

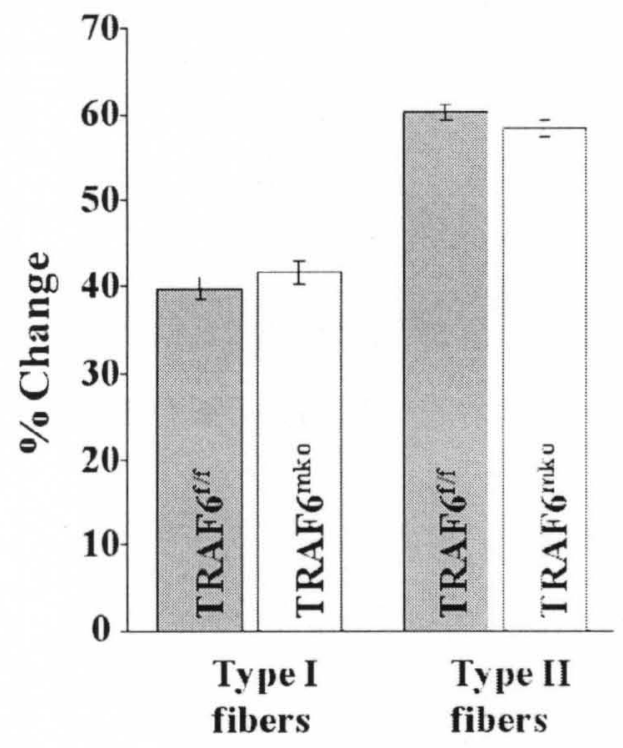

I.

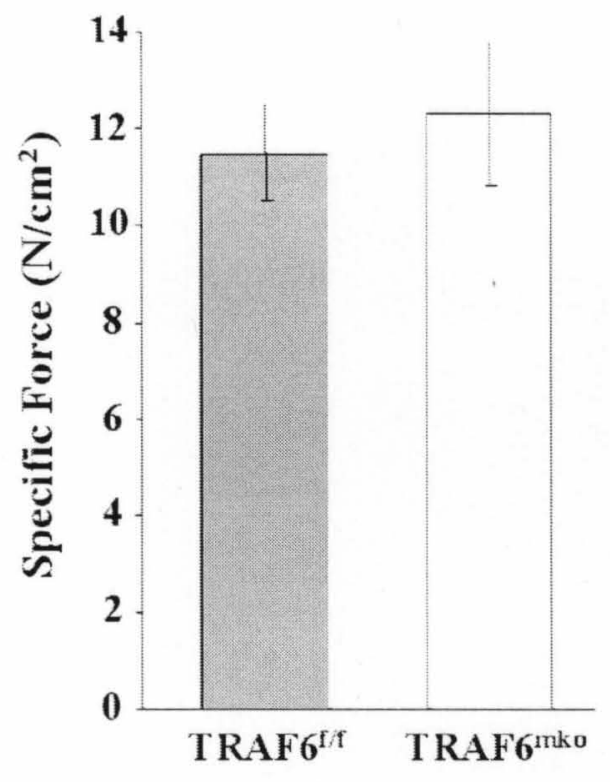


Figure 2.2: Generation of skeletal muscle-specific TRAF6 knockout mice. (A) Strategy for the generation of TRAF $6^{\text {mko }}$ mice. TRAF $6^{\mathrm{f} / \mathrm{f}}$ mice were generated by homologous recombination of a $15-\mathrm{kb}$ DNA fragment containing a $5.5-\mathrm{kb}$-long homologous (LH) fragment, loxP site, 2-kb TRAF6 exon, 1.4-kb stop cassette, loxP site, 1.5-kb IRES-YFP, and 1.5-kb short homologous ( $\mathrm{SH}$ ) fragment. These mice were crossed with MCK-Cre mice to delete the floxed exon. (B) Drawing depicting the breeding strategy for generation of TRAF6 ${ }^{\mathrm{mko}}$ and littermate TRAF6 ${ }^{\mathrm{t} / \mathrm{f}}$ mice. (C) Representative photomicrographs of the semiquantitative reverse polymerase PCR gels showing reduced expression of TRAF6 in TA muscle of TRAF $6^{\text {mko }}$ mice compared with TRAF $6^{\mathrm{f} / \mathrm{f}}$ or C57BL6 mice. The levels of IRES and GAPDH were comparable between TRAF6 ${ }^{\mathrm{f} / \mathrm{f}}$ and TRAF6 ${ }^{\text {mko }}$ mice. (D) Western blot analysis of TRAF6, TRAF3, TRAF4, TAB1, and tubulin protein levels showed depletion of TRAF6 only in skeletal muscle of TRAF ${ }^{\text {mko }}$ mice. (E) Morphometric analysis of H\&E stained TA muscle sections demonstrate that (F) There is no significant difference in mean CSA and (G) Number of myofibers were comparable in TRAF6 ${ }^{\mathrm{f} / \mathrm{f}}$ and TRAF6 ${ }^{\mathrm{mko}}$ mice. (H, I and $\left.\mathbf{J}\right) \mathrm{TRAF}^{\mathrm{f} / \mathrm{f}}$ and $\mathrm{TRAF}^{\mathrm{mko}}$ were found comparable in (H) Serum creatine-kinase acivity (I) Specific force produced by soleus muscle and $(\boldsymbol{J})$ Fiber type composition in soleus muscle. 

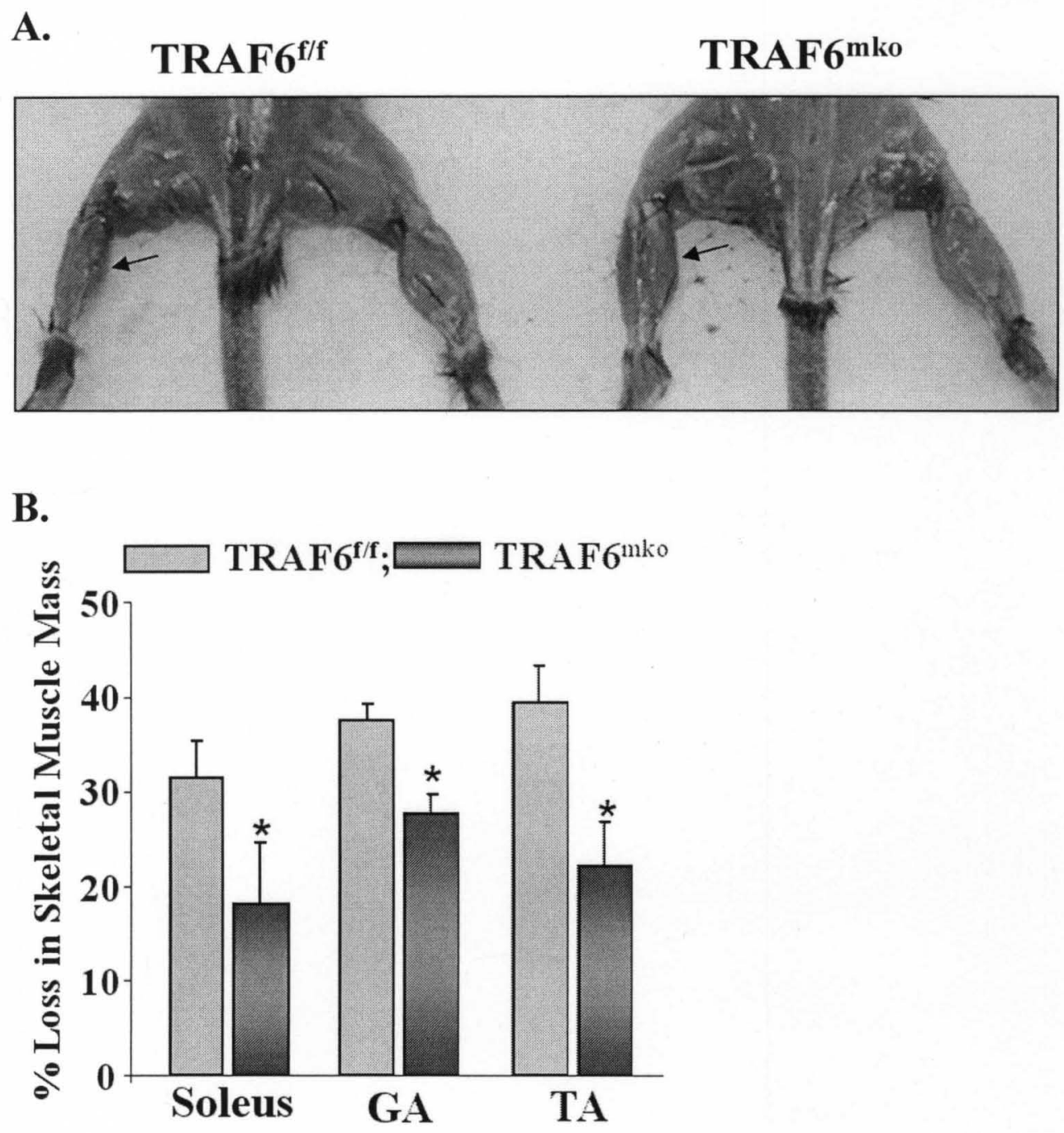
C.

TA muscle

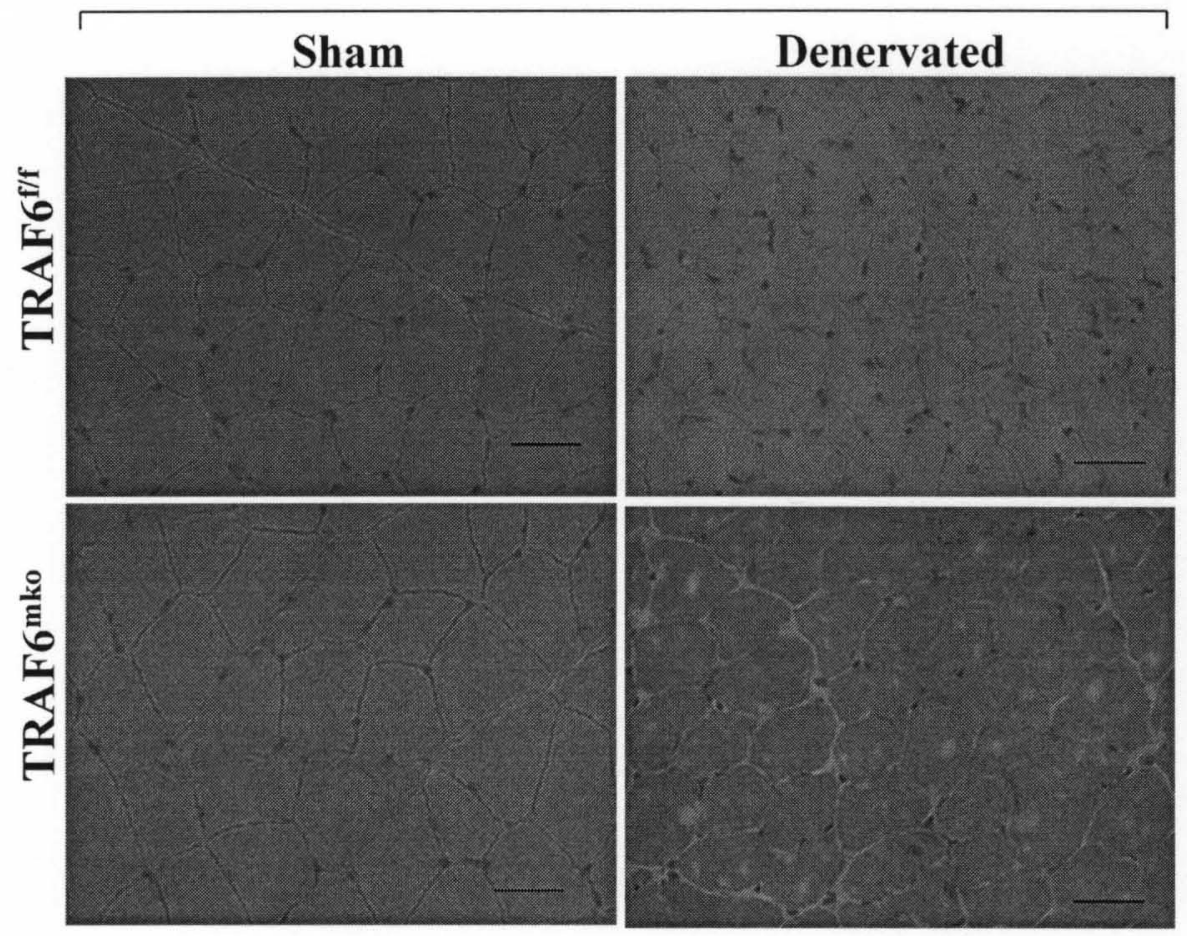

D.

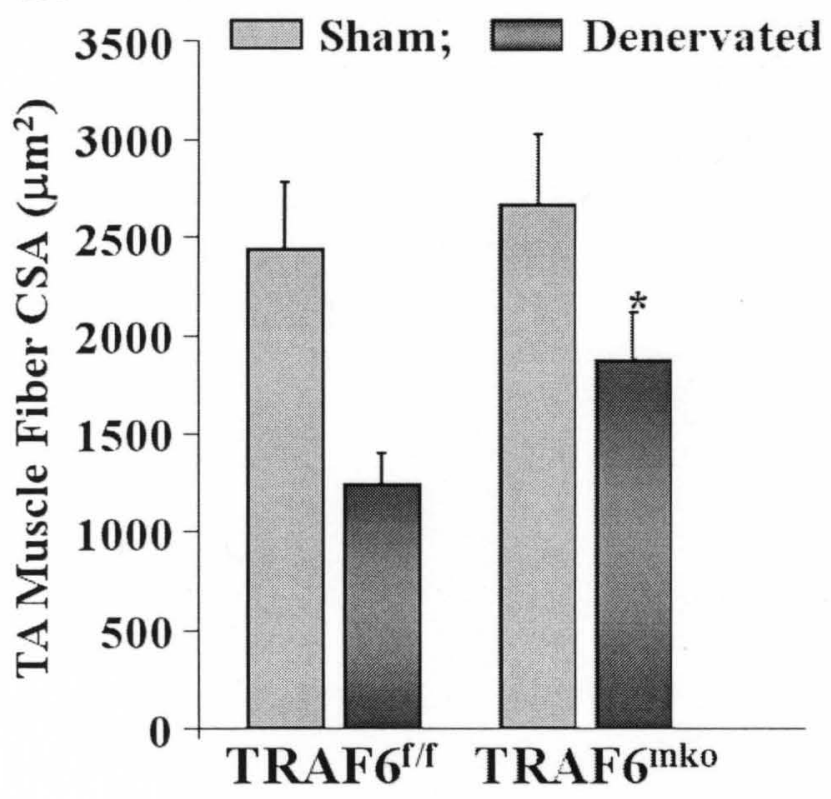


E.

Soleus muscle

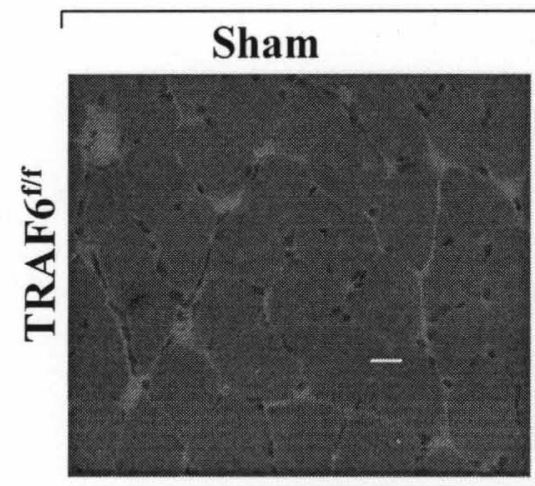

Denervated
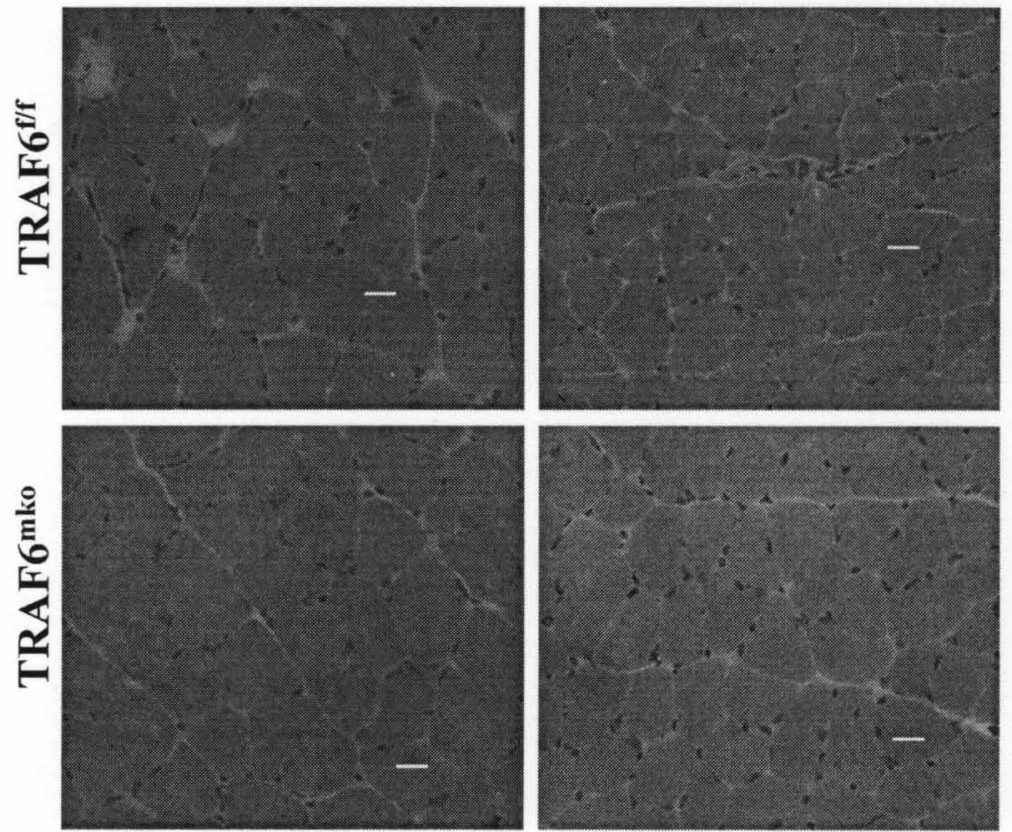

F.

TRAF $6^{\text {mko }}$

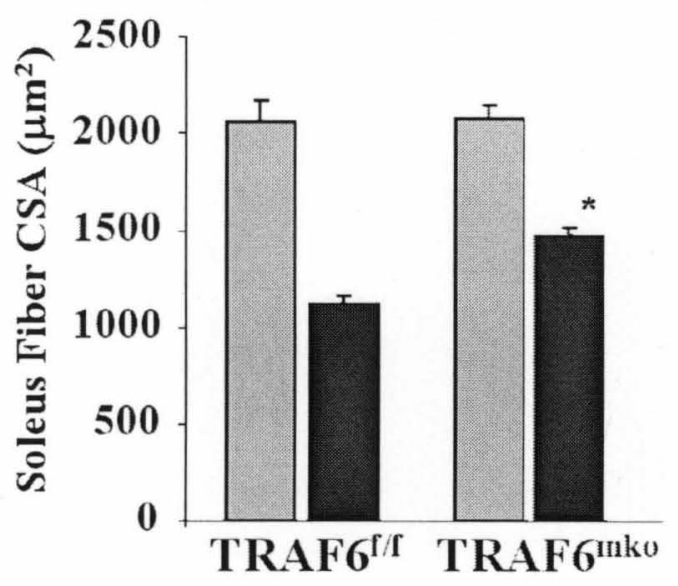




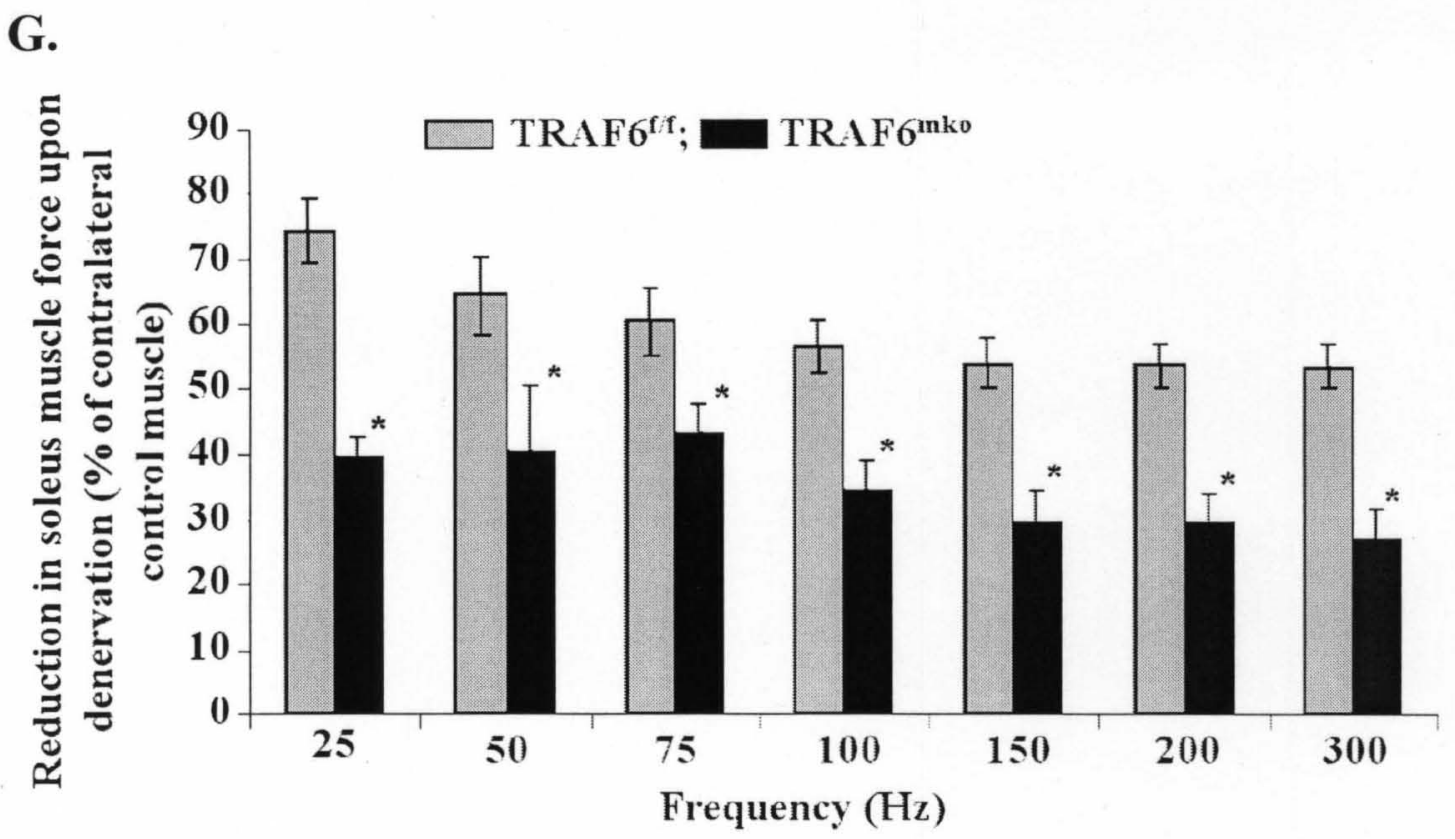

Figure 2.3. Ablation of TRAF6 prevents denervation-induced muscle loss in mice. 3mo-old TRAF $6^{\mathrm{f} / \mathrm{f}}$ and TRAF6 ${ }^{\text {mko }}$ mice were denervated by transection of sciatic nerve. (A) Arrows point to GA muscle $14 \mathrm{~d}$ after denervation. (B) TA, GA, and soleus muscle were isolated tendon to tendon from TRAF $6^{\mathrm{f} / \mathrm{f}}$ and TRAF $6^{\mathrm{mko}}$ mice $14 \mathrm{~d}$ after denervation ( $\mathrm{n}=8$ per group), and their wet weight was measured. (C) H\&E-stained sections of TA muscle of TRAF $6^{\mathrm{f} / \mathrm{f}}$ and TRAF $6^{\mathrm{mko}}$ mice $14 \mathrm{~d}$ after denervation. Bars, $50 \mu \mathrm{m}$. (D) Quantification of mean fiber CSA of TA muscle in TRAF6 ${ }^{\mathrm{f} / \mathrm{f}}$ and TRAF6 ${ }^{\mathrm{mko}}$ mice $14 \mathrm{~d}$ after denervation ( $\mathrm{n}=8$ in each group). (E) Representative H\&E-stained sections of soleus muscle of TRAF6 ${ }^{\mathrm{f} / \mathrm{f}}$ and TRAF $6^{\mathrm{mko}}$ mice. Bars, $20 \mu \mathrm{m}$. (F) Quantification of fiber CSA in soleus muscle in TRAF $6^{\mathrm{f} / \mathrm{f}}$ and TRAF $6^{\text {mko }}$ mice after denervation $(\mathrm{n}=8$ in each group). (G) Denervation-induced loss in absolute muscle force production in isometric contraction was measured in soleus muscle of $\mathrm{TRAF}^{\mathrm{f} / \mathrm{f}}(\mathrm{n}=4)$ and TRAF6 ${ }^{\text {mko }}(\mathrm{n}=4)$ mice at the indicated frequencies. Error bars represent SD. *, P $<0.05$ (values significantly different from denervated muscle of TRAF $6^{\mathrm{f} / \mathrm{f}}$ mice). 

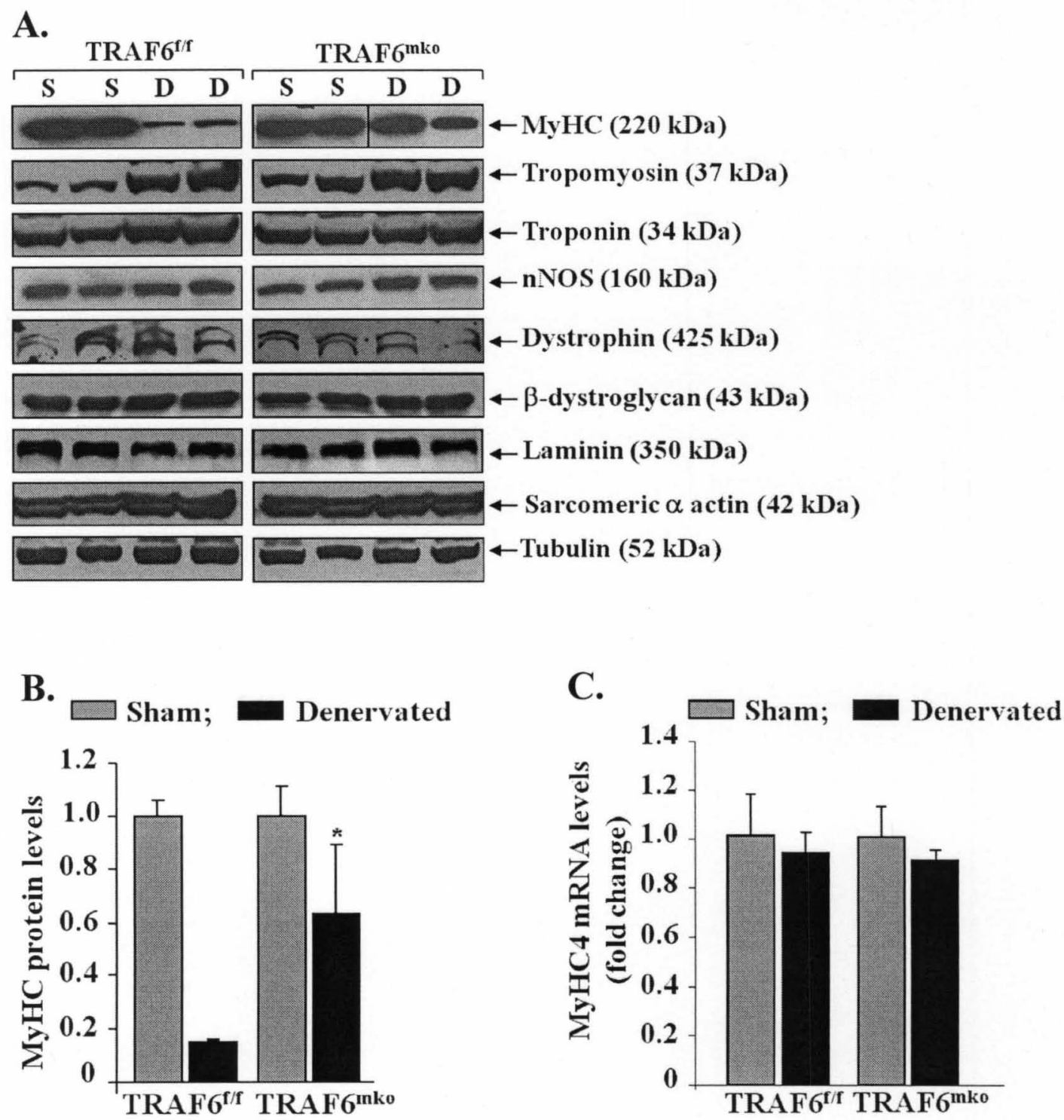

Figure 2.4. Depletion of TRAF6 prevents degradation of specific muscle proteins in denervated skeletal muscle. (A) Representative immunoblots for $\mathrm{MyHC}$, tropomyosin, troponin, nNOS, dystrophin, $\beta$-dystroglycan, sarcomeric $\alpha$-actin, laminin, and tubulin in TA muscle $10 \mathrm{~d}$ after denervation. Black lines indicate that intervening lanes have been spliced out. (B) Fold change in protein levels of MyHC in TA muscle of TRAF6 $6^{\mathrm{fff}}(n=4)$ and TRAF6 ${ }^{\text {mko }}(n=4)$ mice after denervation. (C) Fold change in mRNA level of MyHC in TA muscle of TRAF6 ${ }^{\mathrm{fff}}(n=4)$ and $\mathrm{TRAF}^{\mathrm{mko}}(n=4)$ mice upon denervation. Error bars represent $\mathrm{SD} .{ }^{*}, \mathrm{P}<0.05$ (values significantly different from TRAF $6^{\mathrm{t} / \mathrm{f}}$ mice). Black lines indicate that intervening lanes have been spliced out. 


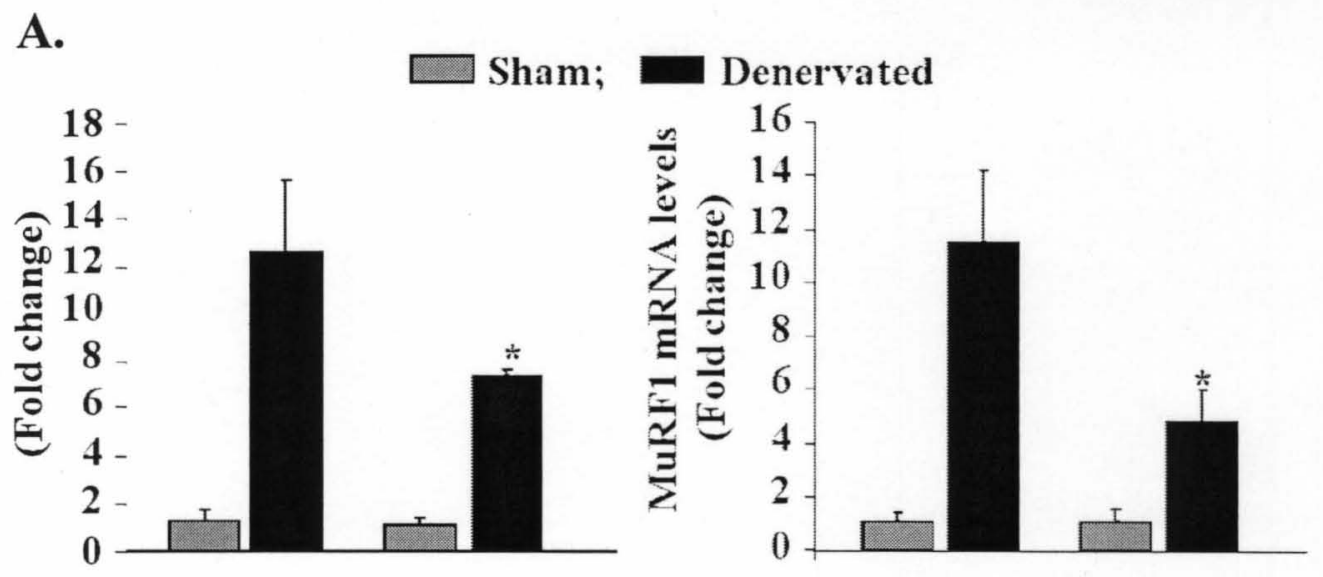

B.
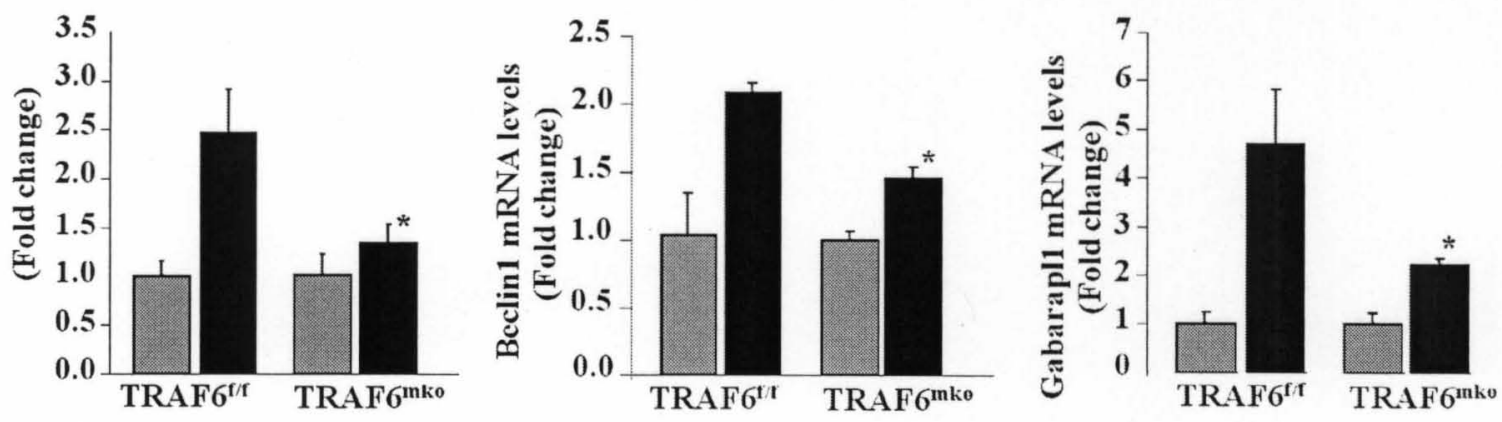

C.

\section{TRAF6 $^{\text {f/f }}$ TRAF6 $^{\text {mko }}$}

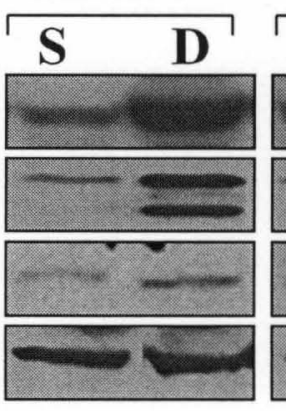

$$
\begin{array}{|l|l|l}
\hline S \quad D & \\
\hline & & \text { MuRF1 (40 kDa) } \\
\hline & & \leftarrow \text { LC3B-I (14 kDa) } \\
\hline & \leftarrow \text { Beclin-1 (60 kDa } \\
\hline & & \text { Tubulin (52 kDa) }
\end{array}
$$


D.

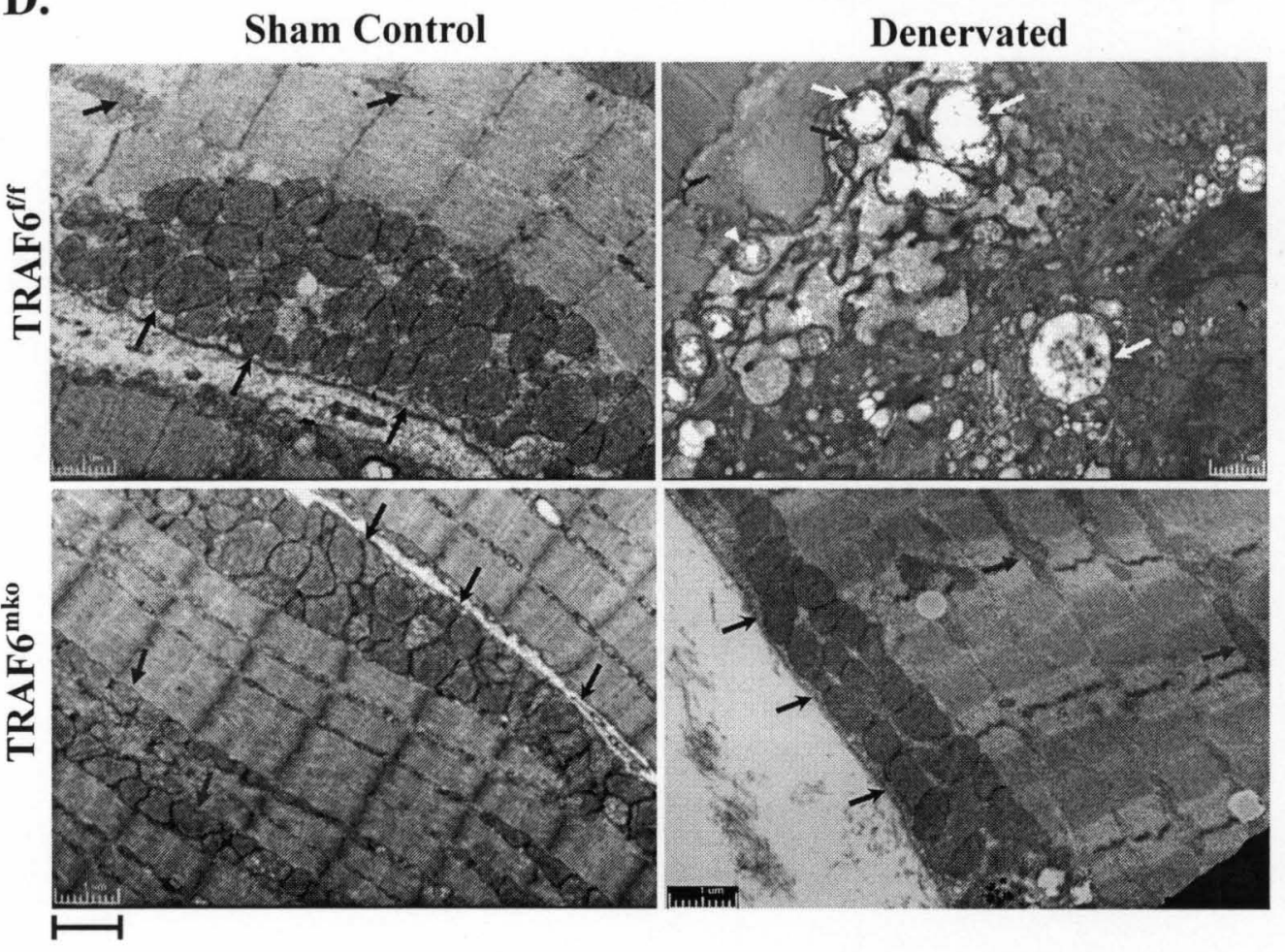

Figure 2.5. TRAF6 is required for the activation of the ubiquitin-proteasome and autophagy systems in denervated skeletal muscle. 3 -mo-old TRAF $6^{\mathrm{f} / \mathrm{f}}$ and TRAF $6^{\mathrm{mko}}$ mice were subjected to denervation for $10 \mathrm{~d}$, and TA muscles were isolated for biochemical analyses. (A) Transcript levels of MAFbx and MuRF1 (measured by QRTPCR assays) were significantly lower in denervated TA muscle of TRAF6 ${ }^{\text {mko }}$ mice compared with TRAF6 ${ }^{\mathrm{f} / \mathrm{f}}$ mice. (B) The expression levels of autophagy-related genes LC3B, Beclin1, and Gabarapl1 were also significantly reduced in denervated TA muscle of TRAF $6^{\mathrm{mko}}$ mice compared with $\mathrm{TRAF}^{\mathrm{f} / \mathrm{f}}$ mice. Error bars represent SD. ${ }^{*}, \mathrm{P}<0.05$ (values significantly different from those of denervated TA muscle of $\mathrm{TRAF}^{\mathrm{f} / \mathrm{f}}$ mice). (C) Representative immunoblots presented here demonstrate reduced protein levels of MuRF1, LC3B, and Beclin1 in denervated TA muscle of TRAF6 ${ }^{\text {mko }}$ mice compared with $\mathrm{TRAF}^{\mathrm{f} / \mathrm{f}}$ mice. Black lines indicate that intervening lanes have been spliced out. (D) Analyses of longitudinal sections of control and denervated TA muscle of TRAF $6^{\mathrm{f} / \mathrm{f}}$ and TRAF $6^{\text {mko }}$ mice using transmission electron microscopy. Black arrows point to subsarcolemmal mitochondrial distribution, blue arrows point to intermyofibrillar mitochondria, white arrows point to autophagosomes, pink arrows point to autophagic vacuoles, and red arrows point to mitochondria being engulfed by autophagosome. Bar, 1 $\mu \mathrm{m}$. 

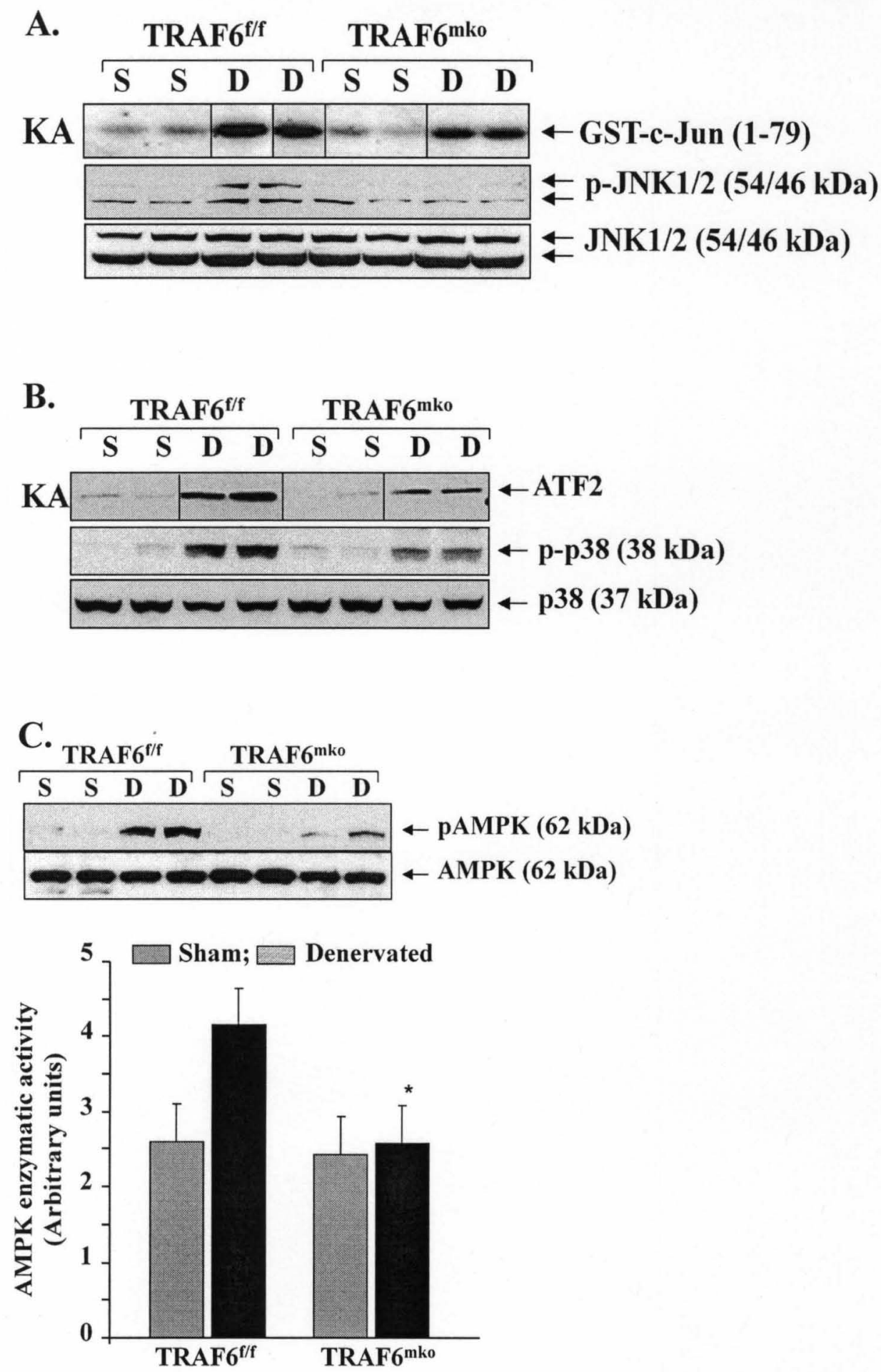

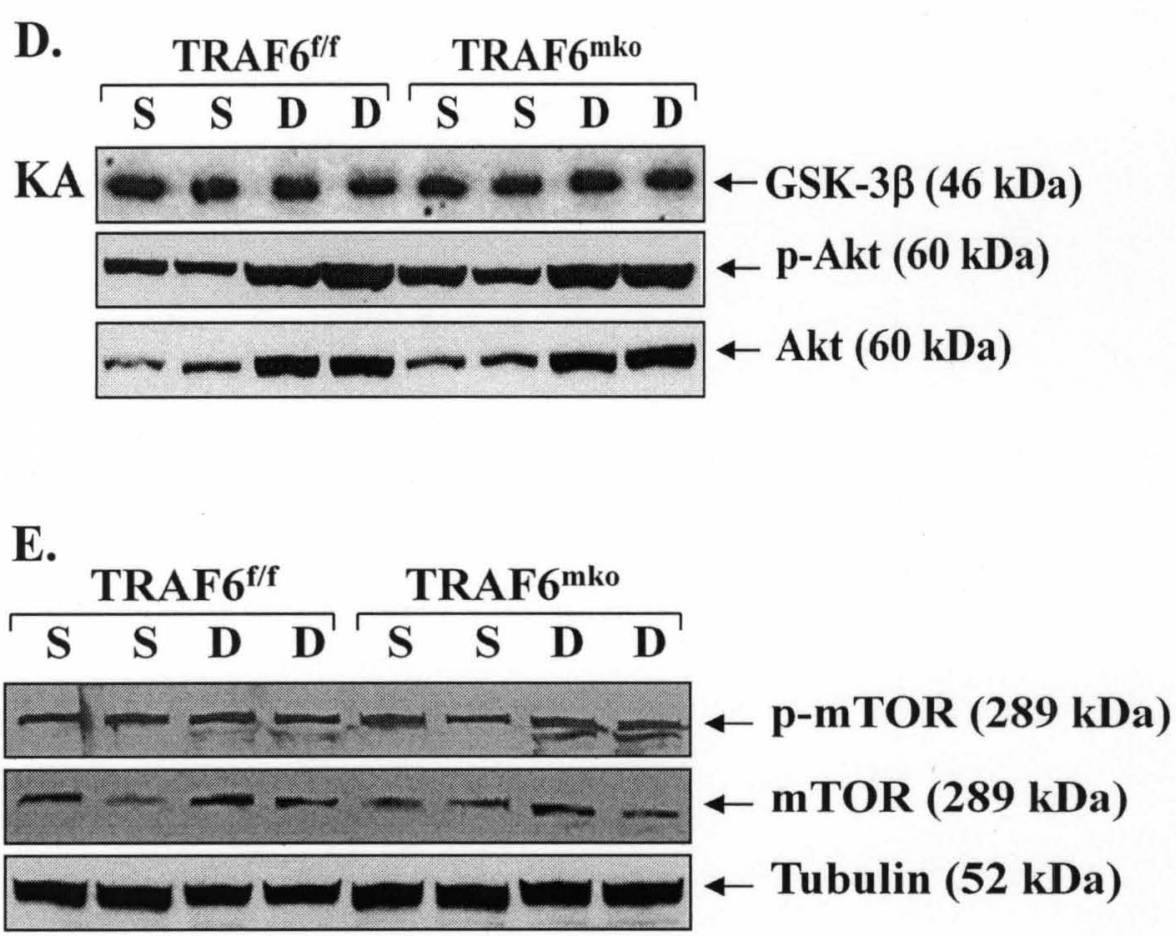

Figure 2.6. Activation of different signaling proteins in denervated muscle of TRAF6 $^{\mathrm{f} / \mathrm{f}}$ and TRAF6 ${ }^{\mathrm{mko}}$ mice. Protein extracts prepared from control or 7 -d postdenervated muscle of TRAF $6^{\mathrm{f} / \mathrm{f}}$ and TRAF $6^{\mathrm{mko}}$ mice were used for in vitro kinase assays or Western blotting. (A) Representative gel pictures show kinase activity, and phosphorylated and total JNK1/2 protein levels in control and denervated TA muscle of TRAF6 ${ }^{\text {mko }}$ and TRAF6 $6^{\mathrm{f} / \mathrm{f}}$ mice. (B) Kinase activity and phosphorylated and total p38 MAPK levels in TA muscle of TRAF $6^{\mathrm{mko}}$ and TRAF6 $6^{\mathrm{f} / \mathrm{f}}$ mice. (C) Gel pictures showing levels of phosphorylated and total AMPK protein in TA muscle of TRAF6 ${ }^{\text {mko }}$ and TRAF6 $^{\mathrm{f} / \mathrm{f}}$ mice (top). Kinase activity of AMPK (bottom) was measured using a commercially available kit. Error bars represent SD. ${ }^{*}, \mathrm{P}<0.05$ (values significantly different from those of denervated TA muscle of TRAF $6^{\mathrm{f} / \mathrm{f}}$ mice). Black lines indicate that intervening lanes have been spliced out. (D) Kinase activity and phosphorylated and total Akt protein levels in TA muscle of $\mathrm{TRAF}^{\mathrm{mko}}$ and $\mathrm{TRAF}^{\mathrm{f} / \mathrm{f}}$ mice. (E) Phosphorylated and total mTOR protein levels in TA muscle of TRAF $6^{\text {mko }}$ and TRAF $6^{\mathrm{f} / \mathrm{f}}$ mice. D, denervated; KA, kinase assay; S, sham operated. 


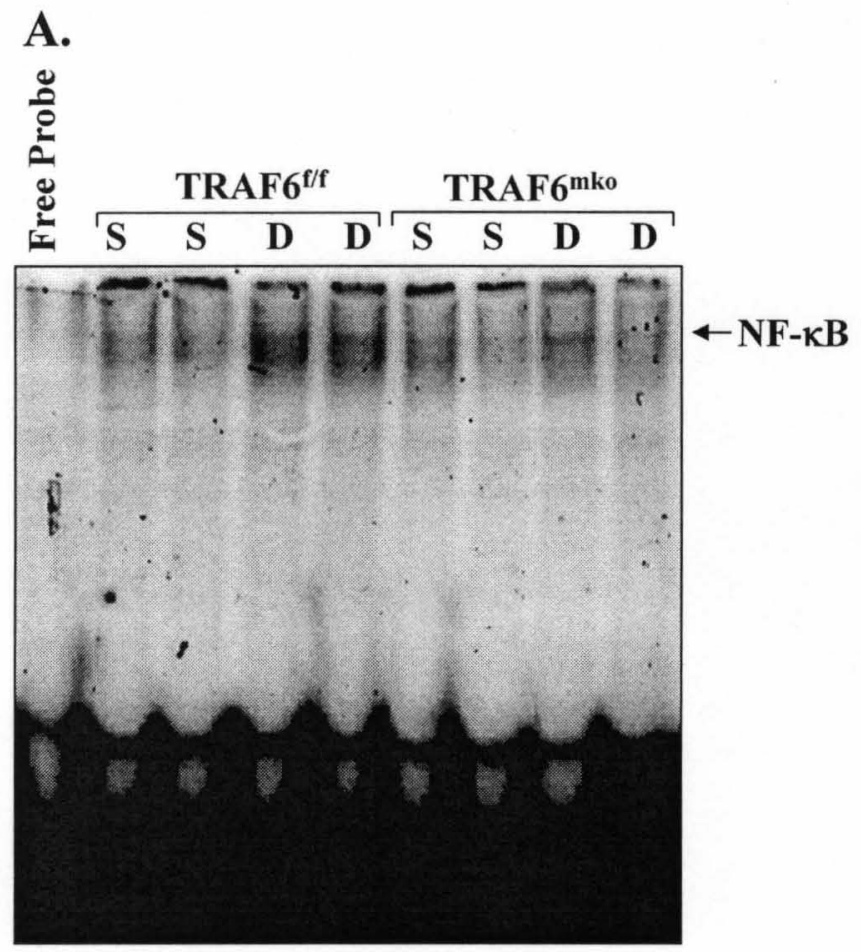

B.

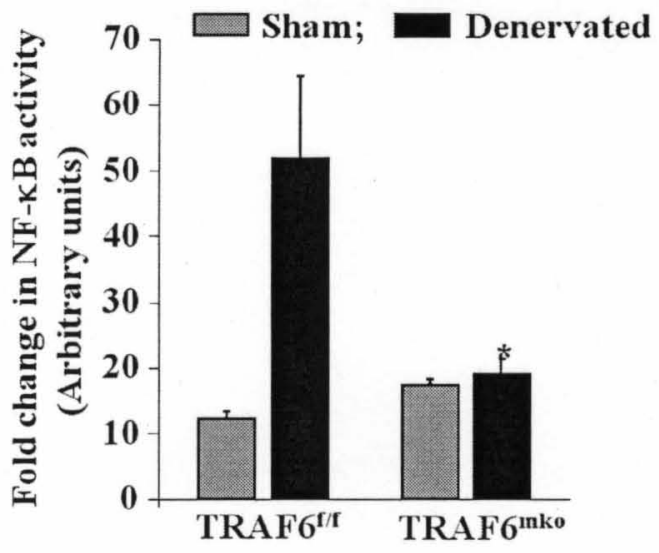

C.

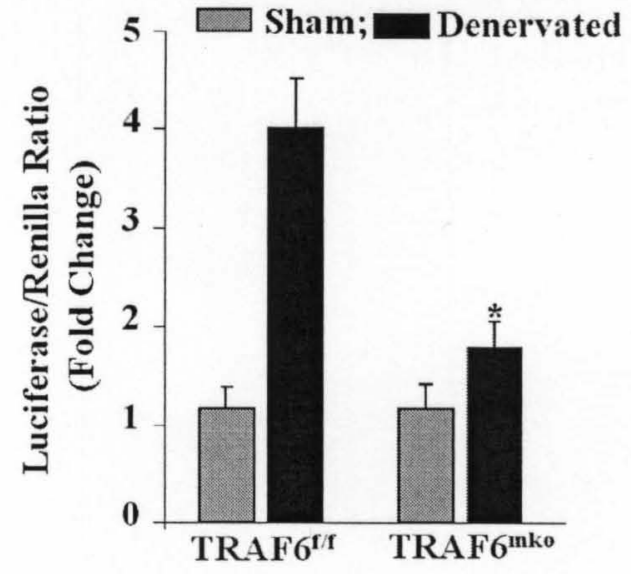




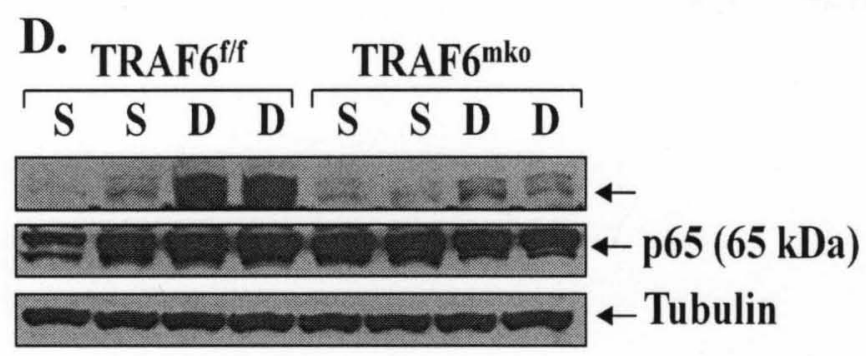

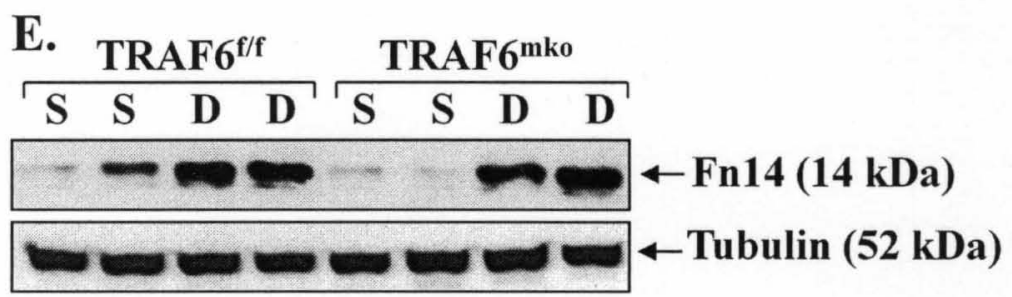

F.

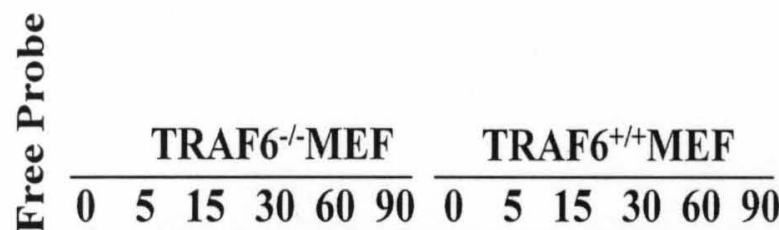

Time of treatment with TWEAK (min)

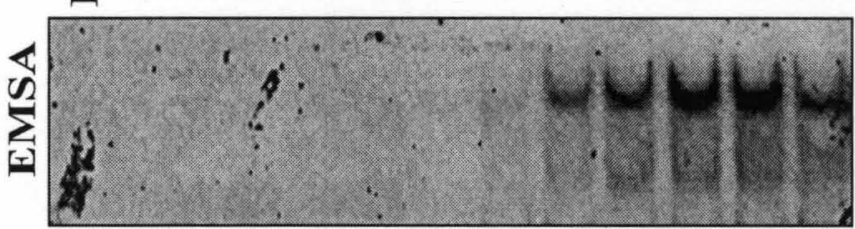

$\leftarrow \mathrm{NF}-\mathrm{kB}$ 


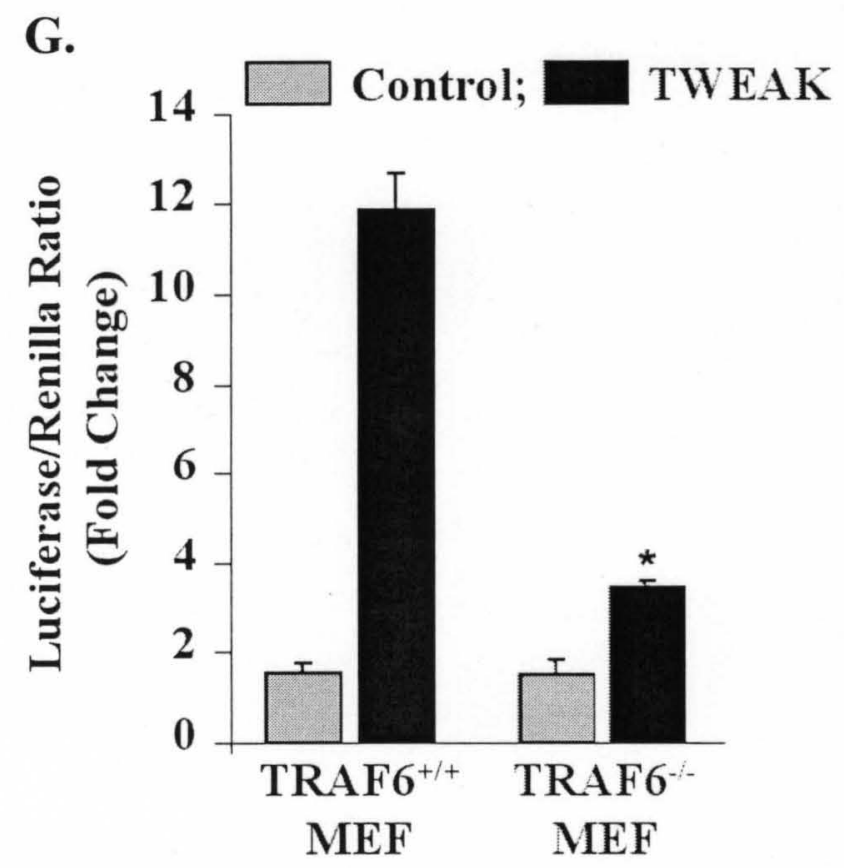

Figure 2.7. TRAF6 is required for the activation of NF- $\mathrm{B}$ transcription factor in denervated skeletal muscle. (A) DNA-binding activity of NF- $\kappa \mathrm{B}$ measured by EMSA in TA muscle of TRAF $6^{\mathrm{f} / \mathrm{f}}$ and TRAF $6^{\text {mko }}$ mice $10 \mathrm{~d}$ after denervation. A representative EMSA gel from three independent experiments is presented. (B) Quantification of fold change in DNA-binding activity of NF- $\kappa \mathrm{B}$ in TA muscle of TRAF6 ${ }^{\mathrm{f} / \mathrm{f}}(n=6)$ and TRAF6 ${ }^{\text {mko }}(n=6)$ mice. (C) Fold change in NF- $\kappa B$ reporter gene activity (normalized using Renilla luciferase) in TA muscle of TRAF6 ${ }^{\mathrm{f} / \mathrm{f}}$ and TRAF $6^{\mathrm{mko}}$ mice in response to denervation. (D) Western blot analyses of the phosphorylated and total form of p65 protein in TA muscle of TRAF $6^{\mathrm{f} / \mathrm{f}}$ and TRAF $6^{\text {mko }}$ mice. (E) Western blot analysis of Fn14 protein in TA muscle of TRAF $6^{\mathrm{f} / \mathrm{f}}$ and $\mathrm{TRAF}^{\mathrm{mko}}{ }^{\mathrm{T}}$ mice $4 \mathrm{~d}$ after denervation. (F) $\mathrm{TRAF}^{+/+}$and $\mathrm{TRAF}^{-/-} \mathrm{MEF}$ were treated with $100 \mathrm{ng} / \mathrm{ml}$ TWEAK for the indicated time intervals, and the activation of $\mathrm{NF}-\kappa \mathrm{B}$ was studied by EMSA. A representative EMSA gel from two independent experiments is presented. (G) Fold change in NF- $\kappa$ B reporter gene activity (normalized using Renilla luciferase) in TRAF6 $6^{+/+}$and TRAF6 ${ }^{-1-}$ MEFs measured after $24 \mathrm{~h}$ of $100 \mathrm{ng} / \mathrm{ml}$ TWEAK treatment. Error bars represent SD. D, denervated; $\mathrm{S}$, sham-operated. *, $\mathrm{P}<0.01$ (values significantly different from denervated TA muscle of TRAF $6^{\mathrm{f} / \mathrm{f}}$ mice). 

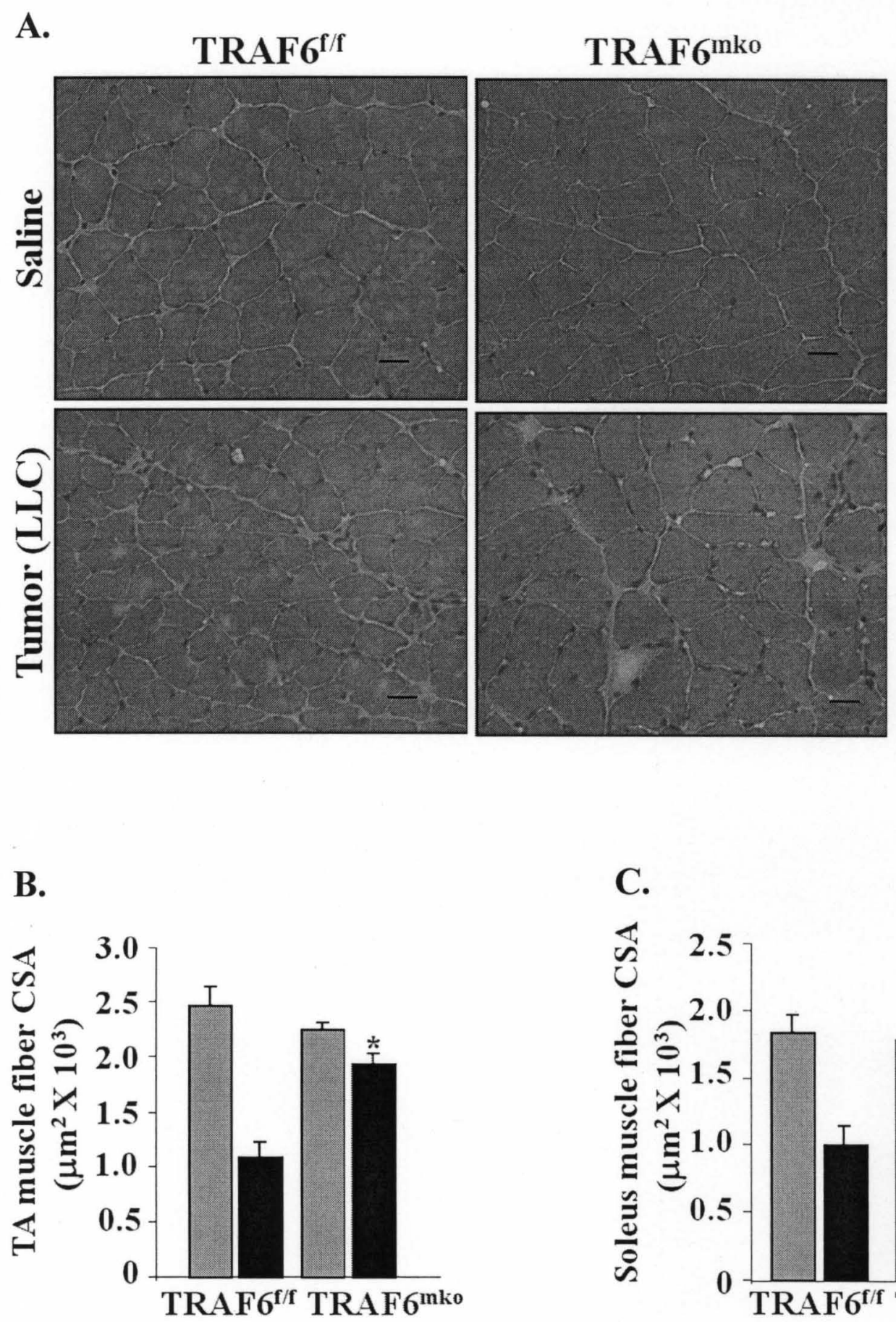

C.

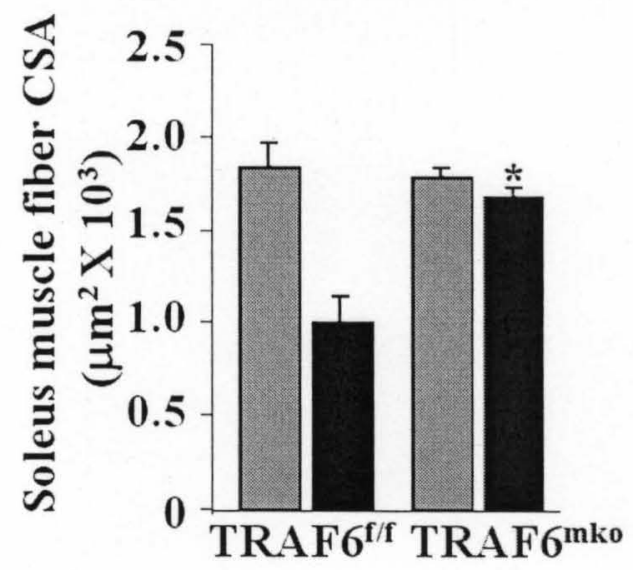



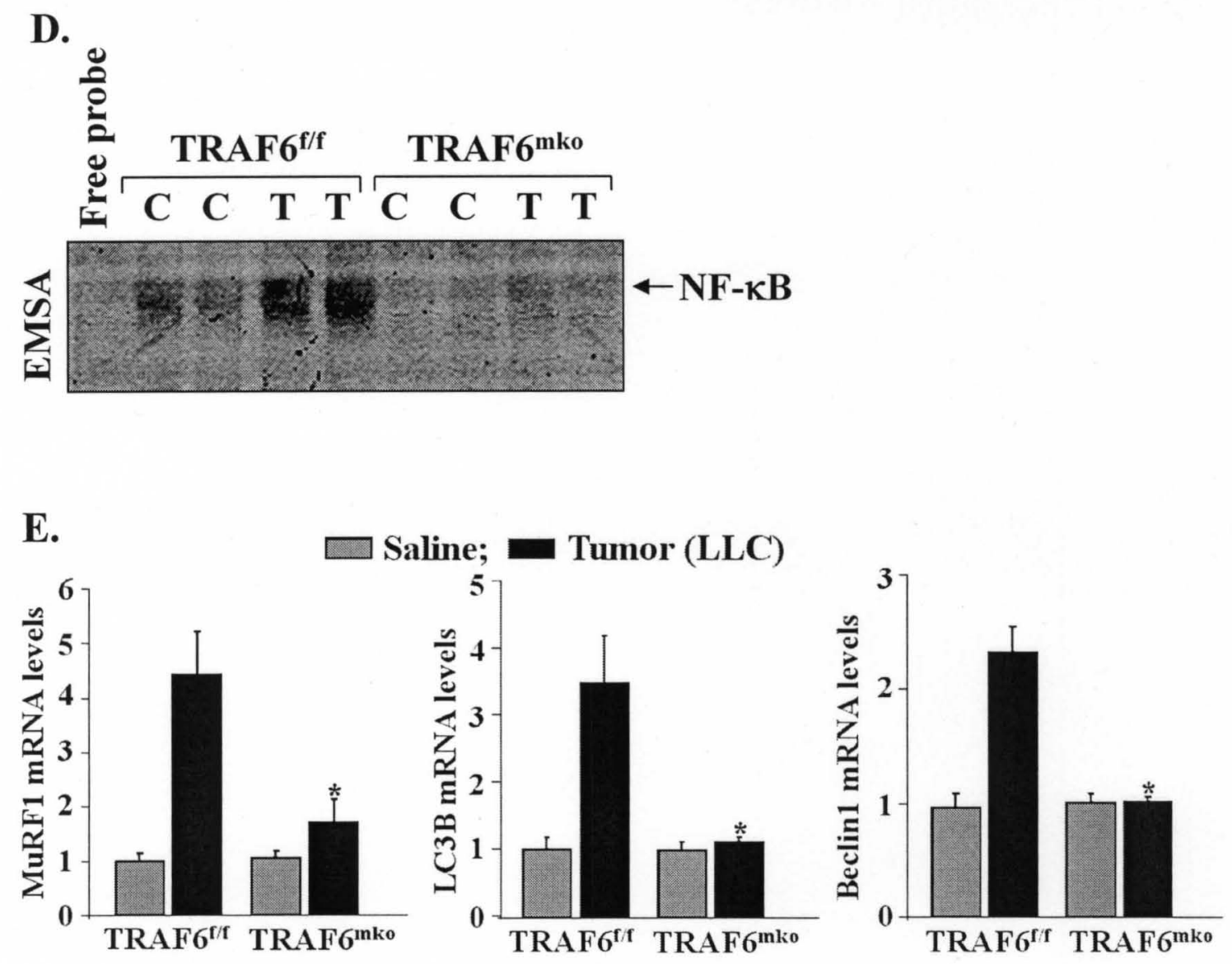

Figure 2.8. Depletion of TRAF6 prevents tumor-induced muscle loss in mice. LLC cells $\left(2 \times 10^{6}\right.$ cells in $100 \mu \mathrm{l}$ saline $)$ were injected in the left flank of TRAF6 $6^{\mathrm{f} / \mathrm{f}}$ and $\mathrm{TRAF}^{\mathrm{mko}}$ mice. Control mice received $100 \mu \mathrm{l}$ of saline only. (A) TA muscles were isolated from control and tumor-bearing mice after $14 \mathrm{~d}$ and analyzed by staining with H\&E. Representative photomicrographs presented here demonstrate that fiber CSA was preserved in TRAF6 ${ }^{\mathrm{mko}}$ mice $(n=7)$ compared with TRAF6 ${ }^{\mathrm{f} / \mathrm{f}}$ mice $(n=6)$. Bars, $20 \mu \mathrm{m}$. (B) Quantification of mean fiber CSA in TA and soleus muscle of TRAF6 ${ }^{\mathrm{f} / \mathrm{f}}$ and TRAF ${ }^{\text {mko }}$ mice after $14 \mathrm{~d}$ of tumor inoculation ( $n=6$ in each group). (C) Analysis of DNA-binding activity of NF- $\mathrm{KB}$ in TA muscle of control and LLC-inoculated TRAF6 6 f/f $(n=4)$ and TRAF6 ${ }^{\text {mko }}$ mice $(n=4)$. A representative EMSA gel is presented. (D) QRTPCR analysis of mRNA levels of MuRF1, LC3B, and Beclin1 in TA muscle of $\mathrm{TRAF}^{\mathrm{mko}}$ and $\mathrm{TRAF}^{\mathrm{t} / \mathrm{f}}$ mice in response to LLC growth $(n=6$ in each group). Error bars represent SD. $*, P<0.01$ (values significantly different from $\mathrm{TRAF}^{\mathrm{f} / \mathrm{f}}$ mice inoculated with LLC). 


\section{CHAPTER THREE}

\section{TRAF6 E3 UBIQUITIN LIGASE INTERCEDES STARVATION-INDUCED MUSCLE ATROPHY THROUGH MULTIPLE MECHANISMS}

\subsection{INTRODUCTION}

Skeletal muscle atrophy that occurs in response to fasting/nutritional deprivation has several common as well as distinct features (3). Like other atrophic programs, the loss of muscle mass upon starvation involves the activation of ubiquitin-proteasome system (UPS) and autophagy-lysosomal system (ALS) $(82,84)$. However, a degree of distinction in starvation-induced muscle atrophy is introduced by the fact that in case of nutrient deprivation, muscle proteins are degraded and mobilized for amino acid production which is further used for gluconeogenesis. In addition, other evidences suggest the involvement of distinct stimuli in starvation-induced catabolic changes such as altered levels of insulin growth factors (IGFs) and glucocorticoids $(22,114,115)$. Recently, activating transcription factor 4 (ATF4) has been reported to be involved in starvation-induced loss of skeletal muscle mass (23). Furthermore, while muscle wasting in response to cancer cachexia or disuse conditions (e.g. denervation, unloading, immobilization etc) involves the activation of transcription factor nuclear factor-kappa B (NF-kB), there has been no evidence regarding the activation or involvement of NF- $\mathrm{kB}$ in the loss of skeletal muscle in response to starvation $(47,102)$. 
TRAF6 is an E3 ubiquitin ligase and through association with the dimeric ubiquitinconjugating enzyme Ubc13/Uev1A, it catalyzes lysine 63 (K63)-linked polyubiquitination of several target proteins $(39,64,116)$. Although it remains enigmatic whether the E3 ubiquitin ligase activity of TRAF6 is essential for its signaling function in all conditions, recent studies have shown that TRAF6 functions as a central regulator in multiple signaling pathways such as NF- $\mathrm{B}$, mitogen-activated protein kinase (MAPK), and phosphatidylinositol 3-kinase (PI3K)/Akt in response to various cytokines and microbial products $(10,30,39,75,76,116)$. In addition to its association with cytoplasmic domains of various cell surface receptors such as toll-like receptors and interleukin-1 receptor, TRAF6 has also been found to interact with multiple components of UPS and/or ALS in some cell types $(21,48,77,93)$. We have also recently reported that TRAF6 regulates skeletal muscle mass and activation of ALS and UPS in denervated skeletal muscle (117). However, the role and the mechanisms of action of TRAF6 and whether E3 ubiquitin ligase activity of TRAF6 is required for muscle atrophy in response to fasting remain unknown.

Several cellular stress conditions, such as starvation and alterations in glycosylation status, lead to accumulation of unfolded and/or misfolded proteins in the ER lumen and cause ER stress $(29,32)$. The ER responds by activation of a range of signaling pathways that are collectively termed the ER stress response or the unfolded protein response (UPR), which is essentially a cyto-protective response, but excessive or prolonged UPR can produce deleterious effects including cell death (118). UPR has three distinct arms which have their specific transducers. Activation of these arms is mediated by PERK (protein kinase RNA (PKR)-like ER kinase), IRE1 (inositol-requiring protein- 
1), and ATF6 (activating transcription factor 6) $(20,29,32)$. Until recently, there was no evidence whether ER stress-related proteins are involved in skeletal muscle metabolism and whether there is any regulatory mediation of ER chaperones in skeletal muscle atrophy. Recently, it has been demonstrated that ATF4, a transcription factor, involved in cellular responses to starvation is involved in muscle atrophy through mechanisms independent of induction of muscle-specific E3 ubiquitin ligases $M A F B x$ (also known as Atrogin-1) and MuRFI (23). It is notable that the expression of ATF4 is increased through the activation of PERK arm of the UPR in addition to other activating kinases such as general control derepressible 2 (GCN2) kinase and heme-regulated inhibitor (HRI) kinase (119). Intriguingly, ER stress responsive transcription factor ATF6 has been implicated in muscle adaptation in response to acute exercise (120). However, direct evidence regarding the activation of UPR in skeletal muscle in atrophic conditions is still lacking.

In this study, using skeletal-muscle specific TRAF6 knockout mice, we have investigated the role and the mechanisms by which TRAF6 regulates starvation-induced muscle atrophy. Our results show that muscle-specific depletion of TRAF6 inhibits starvation-induced activation of UPS and ALS and muscle atrophy in mice. Intriguingly, TRAF6 is essential for the inducible expression of several ER stress response-related genes including ATF4 in response to fasting. Our results also suggest that the TWEAKFn14 system is involved in fasting-induced muscle atrophy and inducible expression of Fn14 in response to starvation requires TRAF6. Finally, our experiments demonstrate that the E3 ubiquitin ligase activity of TRAF6 is essential for induction of atrophic program in skeletal muscle in response to starvation. 


\subsection{MATERIALS AND METHODS}

\section{Mice}

Floxed TRAF6 $\left(\right.$ TRAF6 $\left.^{\mathrm{f} / \mathrm{f}}\right)$ and muscle specific knock-out for TRAF6

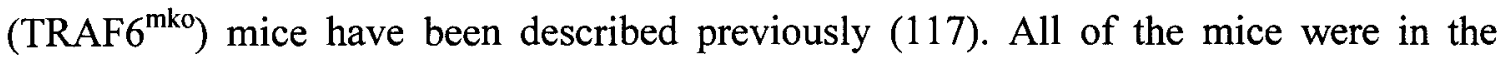
C57BL/6 background, and their genotype was determined by PCR from tail DNA. For starvation studies, mice were provided water but kept unfed for $6 \mathrm{~h}, 12 \mathrm{~h}, 24 \mathrm{~h}$ or $48 \mathrm{~h}$. All experimental protocols with mice were approved in advance by the Institutional Animal Care and Use Committee at University of Louisville.

\section{Cell Culture}

C2C12 cells (a myoblastic cell line) were obtained from American Type Culture Collection. These cells were grown in DMEM containing 10\% FBS. Myoblasts were transfected with different plasmids using Effectene transfection reagent (Qiagen). To induce differentiation, the cells were incubated in differentiation medium $(2 \%$ horse serum in DMEM) for $96 \mathrm{~h}$ as described (117). For starvation studies, the medium of the differentiated myotubes was replaced with sterile phosphate buffered saline (PBS) for $3 \mathrm{~h}$ or $6 \mathrm{~h}$ and the myotubes were examined by morphometric or biochemical assays.

\section{In vivo Gene Delivery}

The injection of plasmid DNA into TA muscle of mice and electroporation were performed according to a protocol as previously described (121). In brief, pcDNA3, FLAG-TRAF6 C70A, and pEGFPC1 were prepared using an endotoxin-free kit (Qiagen) and suspended in sterile saline solution in a 1:10 ratio. Mice were anesthetized, and a small portion of TA muscle of both hind limbs was surgically exposed and injected with 
$30 \mu \mathrm{l}$ of $0.5 \mathrm{U} / \mu \mathrm{l}$ hyaluronidase (EMD Biosciences). After $2 \mathrm{~h}$, plasmid DNA ( $30 \mu \mathrm{g}$ in 25 $\mu l$ saline) was injected in TA muscle, and 1 min after plasmid DNA injection, a pair of platinum plate electrodes were placed against the closely shaved skin on both sides, and electric pulses were delivered. Three $20 \mathrm{~ms}$ square-wave pulses of $1-\mathrm{Hz}$ frequency at $75 \mathrm{~V} / \mathrm{cm}$ were generated using a stimulator (model S88; Grass Technologies) and delivered to the muscle. The polarity was then reversed and a further three pulses were delivered to the muscle. After electroporation, mice were returned to their cages and fed a standard diet. Mice were used 10 days after plasmid electroporation for starvation studies.

\section{Histology and Morphometric Analysis}

Hind limb muscles (soleus and TA) of mice were removed, frozen in isopentane cooled in liquid nitrogen, and sectioned using a microtome cryostat. For the assessment of tissue morphology, 10- $\mu \mathrm{m}$-thick transverse sections of muscles were stained with $\mathrm{H} \& \mathrm{E}$, and staining was visualized (without any imaging medium) at room temperature on a microscope (Eclipse TE 2000-U) using a Plan 10X, NA 0.25 PH1 DL or Plan-Fluor ELWD 20X, NA 0.45 Ph1 DM objective lens, a digital camera (Digital Sight DS-Fi1), and NIS Elements BR 3.00 software (all from Nikon). The images were stored as JPEG files, and image levels were equally adjusted using Photoshop CS2 software (Adobe). Fiber CSA was analyzed in H\&E-stained soleus or TA muscle sections. For each muscle, the distribution of fiber CSA was calculated by analyzing 200-250 myofibers using NIS Elements BR 3.00 software (Nikon) as described (53). 


\section{Immunoprecipitation and Western Blot}

Levels of different proteins in skeletal muscle were determined by performing immunoblotting as described (53). In brief, tissues were washed with PBS and homogenized in Western blot lysis buffer A (50 mM Tris-Cl, pH 8.0, $200 \mathrm{mM} \mathrm{NaCl}, 50$ $\mathrm{mM} \mathrm{NaF}, 1 \mathrm{mM}$ DTT, $1 \mathrm{mM}$ sodium orthovanadate, $0.3 \%$ IGEPAL, and protease inhibitors). Approximately $100 \mu \mathrm{g}$ of protein was resolved on each lane on $10-12 \%$ SDSPAGE, electrotransferred onto nitrocellulose membrane, and probed using anti-TRAF6 (1:1,000; Millipore), anti-TRAF3 (1:1,000; Santa Cruz Biotechnology, Inc.), anti-TRAF5 (1:1,000; Santa Cruz Biotechnology, Inc.), anti-Ubiquitin (1:1,000; Santa Cruz Biotechnology, Inc.), anti-phospho FOXO3a (1:1,000; Cell Signaling Technology), antiphospho Akt (1:200; Cell Signaling Technology), anti-Akt (1:1,000; Cell Signaling Technology), anti-phospho-eIF2 (1:1000; Cell Signaling Technology), anti-eIF2 (1:1,000; Cell Signaling Technology), anti-ATF3 (1:1000; Santa Cruz Biotechnology), anti-ATF4 (1:1000; Santa Cruz Biotechnology), anti-PDI (1:1,000; Santa Cruz Biotechnology), anti-CHOP (1:1,000; Santa Cruz Biotechnology), anti-LC3B (1:1,000; Cell Signaling Technology), anti-p62 (1:1,000; MBL International), anti-Fn14 (1:1,000; Cell Signaling Technology), MF-20 (1:1,000; Development Studies Hybridoma Bank, University of Iowa) and detected by chemiluminescence. The bands were quantified using ImageQuant TL software (GE Healthcare). To study the autoubiquitination of TRAF6, muscle extract (400 $\mu \mathrm{g}$ protein) was incubated overnight with $1 \mu \mathrm{g}$ anti-TRAF6 antibody (MBL) in $600 \mu \mathrm{l}$ of lysis buffer followed by addition of protein A sepharose beads and incubation at $4^{\circ} \mathrm{C}$ for additional $2 \mathrm{~h}$. The beads were washed four times with lysis buffer and finally suspended in 2X Laemmli's sample buffer. Proteins were resolved 
on $10 \%$ SDS-PAGE gel and immunoblotted using Lys 63-specific ubiquitin antibody $(1: 1,000 ;$ Millipore $)$.

\section{Total RNA Extraction and QRT-PCR Assay}

RNA isolation and QRT-PCR were performed using gene specific primers following same method as described (53).

\section{Statistical Analysis}

Results are expressed as mean $\pm \mathrm{SD}$. The Student's $t$ test or analysis of variance was used to compare quantitative data populations with normal distributions and equal variance. A value of $\mathrm{P}<0.05$ was considered statistically significant unless otherwise specified. 


\subsection{RESULTS}

\subsubsection{TRAF6 Mediates the Starvation-Induced Fiber Atrophy in Mice}

We have previously reported that the expression of TRAF6 is increased in skeletal muscle in multiple catabolic conditions including denervation and cancer cachexia (117). We first investigated how the expression of TRAF6 is affected in skeletal muscle of mice in response to fasting. Wild-type mice were given access to normal water but deprived of food for 24h. Transcript levels of TRAF6 in hind limb muscle were measured using quantitative real-time PCR (QRT-PCR) assay. As shown in Figure 3.1 A, mRNA levels of TRAF6 were significantly increased in tibial anterior (TA), gastrocnemius (GA), and soleus muscle of fasted mice compared to controls. We also performed Western blot for a few TRAFs. In TA muscle of unstarved mice, level of TRAF6 protein was much lower compared to TRAF2, TRAF3, or TRAF5. However, the levels of TRAF6 protein were considerably increased within $6 \mathrm{~h}$ and remained elevated even after $24 \mathrm{~h}$ of fasting. In contrast, fasting did not affect protein levels of TRAF2, TRAF3, or TRAF5 in TA muscle of mice (Figure 3.1 B). To identify the physiological significance of increased expression of TRAF6 in fasted muscle, we investigated the effects of deletion of TRAF6 on muscle atrophy in response to starvation. Skeletal muscle-specific TRAF6 knockout (henceforth $\mathrm{TRAF}^{\mathrm{mko}}$ ) and their corresponding littermates (i.e. TRAF6 ${ }^{\mathrm{fff}}$ ) as described (117) were fasted for $24 \mathrm{~h}$ or $48 \mathrm{~h}$. Hind limb muscles isolated were used to prepare transverse cryosections followed by Hematoxylin and Eosin (H\&E) staining and quantification of fiber cross sectional area (CSA). Fasting for $24 \mathrm{~h}$ or $48 \mathrm{~h}$ caused a significant reduction in fiber CSA in TA muscle of TRAF $6^{\mathrm{f} / \mathrm{f}}$ mice (Figure $3.1 \mathrm{C}$ ). However, fasting-induced loss 
in fiber CSA was significantly inhibited in TA muscle of TRAF6 ${ }^{\mathrm{mko}}$ mice (Figure 3.1 C, 3.1 D). Furthermore, the proportion of fibers with larger CSA was notably higher in TA muscle of TRAF $6^{\mathrm{mko}}$ mice compared to littermate $\mathrm{TRAF}^{\mathrm{f} / \mathrm{f}}$ evaluated after $24 \mathrm{~h}$ (Figure 3.1 E) or $48 \mathrm{~h}$ (Figure $3.1 \mathrm{~F}$ ) of fasting. TA muscle of mice contains predominately fasttype fibers. In contrast, soleus muscle contains almost equal proportion of fast and slowtype fibers (53). We also performed H\&E-staining and quantified fiber CSA in soleus muscle of TRAF $6^{\mathrm{f} / \mathrm{f}}$ and $\mathrm{TRAF}^{\mathrm{mko}}$ mice. Similar to TA muscle, fasting-induced loss in fiber CSA was significantly inhibited in soleus muscle of TRAF $6^{\text {mko }}$ mice compared to TRAF6 $^{\mathrm{fff}}$ mice (Figure $3.1 \mathrm{G}, 3.1 \mathrm{H}$ ). These results demonstrate that TRAF6 mediates starvation-induced muscle atrophy in vivo.

\subsubsection{TRAF6 is Required for the Activation of UPS and ALS in Skeletal muscle in Response to Starvation}

UPS is responsible for breakdown of majority of proteins in mammalian cells (122, 123). In distinct catabolic conditions, difference between ubiquitination of total and that of specific proteins can affect the development of pathology (124). To understand the mechanisms by which TRAF6 causes muscle atrophy and to determine whether TRAF6 is involved in the activation of UPS, we first investigated how the conjugation of ubiquitin to muscle proteins is affected in skeletal muscle of fasted $\operatorname{TRAF}^{\mathrm{tff}}$ and TRAF6 $^{\text {mko }}$ mice. Gastrocnemius (GA) muscle extracts prepared from unstarved or $24 \mathrm{~h}$ starved $\mathrm{TRAF}^{\mathrm{f} / \mathrm{f}}$ and $\mathrm{TRAF}^{\mathrm{mko}}$ mice were immunoblotted using antibody against ubiquitin. As shown in Figure 3.2 A, fasting augmented the conjugation of ubiquitin to muscle proteins. However, the fasting-induced increase in protein ubiquitylation was 
considerably lower in TA muscle of TRAF $6^{\mathrm{mko}}$ mice compared with $\mathrm{TRAF}^{\mathrm{f} / \mathrm{f}}$ mice (Figure $3.2 \mathrm{~A}$ ) indicating that TRAF6 is required for the activation of UPS in skeletal muscle upon starvation. While muscle atrophy involves enhanced degradation of several contractile proteins, myosin heavy chain-fast type (MyHCf) is one such protein which undergoes rapid degradation in many catabolic conditions potentially through UPS (47, 53). To further validate that TRAF6 is involved in the degradation of muscle proteins, we measured protein levels of MyHCf by Western blot. As shown in Figure $3.2 \mathrm{~A}$ (middle panel), levels of MyHCf were considerably higher in fasted muscle of TRAF $6^{\text {mko }}$ mice compared to littermate TRAF6 $6^{\mathrm{f} / \mathrm{f}}$.

The activation of UPS in multiple atrophic conditions involves the increased expression of two muscle-specific E3 ubiquitin ligases $M U R F 1$ and $M A F B x(9,27,82)$. To further validate the role of TRAF6 in the activation of UPS in response to starvation, we measured transcript levels of $M U R F 1$ and $M A F B x$ in skeletal muscle of TRAF $6^{\mathrm{f} / \mathrm{f}}$ and TRAF $6^{\text {mko }}$ mice. Fasting significantly increased the expression of both $M A F B x$ and MURF1 in skeletal muscle of mice (Figure $3.2 \mathrm{~B}$ ). However, the increase in transcript levels of both MAFBx and MuRF1 in response to fasting was found to be significantly reduced in TRAF $6^{\mathrm{mko}}$ mice compared to $\mathrm{TRAF} 6^{\mathrm{f} / \mathrm{f}}$ mice (Figure $3.2 \mathrm{~B}$ ).

It has been previously reported that fasting also induces the activation of ALS in skeletal muscle $(13,125)$. Furthermore, there is evidence that ALS coordinates with UPS to stimulate muscle atrophy in diverse conditions including starvation $(13,14,18)$. To evaluate the role of TRAF6 in activation of autophagy, we measured mRNA levels of autophagy-related genes LC3B, Beclin1, and Atg12 which have been previously reported to be upregulated in skeletal muscle in response to fasting (14). As shown in 
Figure 3.2 C, fasting-induced expression of LC3B, Beclin1, and Atg12 was significantly inhibited in skeletal muscle of TRAF ${ }^{\mathrm{mko}}$ mice compared with $\mathrm{TRAF}^{\mathrm{fff}}$ mice.

Conversion of LC3BI to the active form LC3BII is a critical event in autophagy and considered as one of the most reliable markers of autophagosome formation (126). We performed Western blot to determine whether TRAF6 affects the conversion of LC3BI into LC3BII in skeletal muscle upon starvation. As shown in Figure 3.2 D, starvation increased the levels of LC3BII form in skeletal muscle of TRAF $6^{\mathrm{f} / \mathrm{f}}$ mice but not TRAF $6^{\mathrm{mko}}$. We further measured levels of p62/SQSTM1, a multi-domain adaptor protein, which interacts with and is activated by TRAF6 $(48,77)$. p62 binds to LC3 through a $\mathrm{LiR}$ (LC3-interacting region) motif and tethers protein to autophagosomes (127). Levels of p62 were noticeably increased in skeletal muscle of $\mathrm{TRAF}^{\mathrm{t} / \mathrm{f}}$ mice in response to starvation. However, the starvation-induced increase in p62 levels was not observed in skeletal muscle of TRAF6 ${ }^{\mathrm{mko}}$ mice (Figure $3.2 \mathrm{D}$ ). These results are in concert with previous findings suggesting that TRAF6 also acts through p62/LC 3 binding to activate autophagy (48).

We next sought to determine whether ablation of TRAF6 intervenes with upstream regulators of UPS and ALS such as p62, FOXO3a, Akt, and AMPK in skeletal muscle. FOXO3a is a member of forkhead family of transcription factors and is known to induce expression of MAFBx and MuRF1 in atrophying skeletal muscle (41). Akt phosphorylates FOXO family of transcription factors including FOXO3a, which leads to their inactivation through retention in cytosol (128). Using a constitutively active form of FOXO3 with three mutated Akt phosphorylation sites, it has been previously reported that FOXO3 induces atrophy by accelerating proteolysis through activation of autophagy 
(13). Our results showed that starvation led to a marked decrease in basal level of phosphorylation of both Akt and FOXO3a in TA muscle of TRAF6 ${ }^{\mathrm{f} / \mathrm{f}}$ mice (Figure 3.2 E). In contrast, no noticeable decrease in phosphorylation of Akt was observed in $\mathrm{TRAF}^{\mathrm{mko}}$ mice upon starvation (Figure $3.2 \mathrm{E}$ ).

AMPK (5' adenosine monophosphate-activated protein kinase) which is activated in response to increased AMP:ATP ratio is linked to muscle atrophy in multiple conditions including fasting (17). As shown in Figure $3.2 \mathrm{~F}$, fasting induced the activation of AMPK in GA muscle of mice. Furthermore, fasting-induced activation of AMPK was significant inhibited in TA muscle of TRAF $6^{\text {mko }}$ compared to TRAF $6^{\mathrm{f} / \mathrm{f}}$ mice (Figure $3.2 \mathrm{~F}$ ). Taken together, these results suggest that in the conditions of starvation, TRAF6 mediates the activation of both UPS and ALS in skeletal muscle potentially through upstream activation of p62, Akt, FOXO3a, and AMPK.

\subsubsection{TRAF6 augments the expression of ER stress response related genes in skeletal muscle}

ER stress and unfolded protein response (UPR) pathways are employed by cells as a corrective measure to avoid an increment in unfolded protein load. In general, it has been observed that PERK and IRE1 arms of UPR are involved in mediating deleterious effects whereas ATF6 arm mediates adaptive responses. We investigated whether starvation augments the expression of various genes involved in UPR pathways and whether TRAF6 plays a role in their induction in skeletal muscle. Interestingly, a drastic increase in mRNA levels of several markers of UPR such as transcription factors ATF4 and CCAAT/enhancer-binding protein homologous protein (CHOP), ER stress inducible 
enzyme protein disulfide isomerase (PDI), and ER resident chaperons glucose-regulated protein 94 (GRP94), and growth arrest and DNA damage-inducible protein (GADD34) was observed in skeletal muscle of TRAF $6^{\mathrm{tff}}$ mice (Figure $3.3 \mathrm{~A}$ ). More importantly, we found that starvation-induced expression of UPR genes was almost completely blunted in skeletal muscle of $\mathrm{TRAF}^{\mathrm{mko}}$ mice. Western blot analysis also demonstrated that starvation augments protein levels of ATF4 and CHOP in skeletal muscle of TRAF6 ${ }^{\mathrm{f} / \mathrm{f}}$ mice. In contrast, there was no noticeable increase in protein levels of ATF4 or CHOP in GA muscle of fasted TRAF6 ${ }^{\text {mko }}$ mice (Figure $3.3 \mathrm{~B}$ ). To further confirm that TRAF6 $\mathrm{s}$ involved in the induction of ER stress, we subjected cultured $\mathrm{TRAF}^{+/+}$and TRAF6 $6^{-1-}$ MEF to serum starvation for $24 \mathrm{~h}$ and measured the levels of various ER stress related genes. Interestingly, the starvation-induced increase in transcript levels of ATF4, GRP94, GADD34, and CHOP were found to be significantly inhibited in $\mathrm{TRAF}^{-{ }_{-1}}$ MEF compared to TRAF6 ${ }^{+/+}$MEF (Figure 3.3 C). Overexpression of ATF6 in cells causes ER stress response depicted increased expression of various ER chaperons. We also investigated whether ATF6-induced expression of GRP78 and GRP94 was completely blunted in TRAF6 ${ }^{-1}$ MEF compared to $\mathrm{TRAF}^{+/+}$MEF (Figure $3.3 \mathrm{D}$ ). Taken together, these data provide the first evidence that TRAF6 is involved in the induction of ER stress response.

Although fasting induced the expression of ER stress response genes in skeletal muscle, we could not detect any change in the level of phosphorylation of eIF $2 \alpha$ (a downstream phosphorylation target and marker for activation of PERK arm of UPR) or splicing of XBP-1 mRNA (a marker for activation of IRE1 arm of UPR) in skeletal muscle in vivo. A recently published study also reported no changes in the level of 
phosphorylation of eIF $2 \alpha$ in skeletal muscle of rat measured after $1 d, 2 d$ or $3 d$ of fasting (16). While there is possibility that the expression of many of these genes (i.e. ATF4, CHOP, GADD34, and PDI) is governed through mechanisms independent of ER stress response, it is also possibility that eIF2 $\alpha$ is activated transiently only at specific time point after starvation. To further examine whether fasting induces ER stress response in skeletal muscle, we employed cultured myotubes and studied the phosphorylation of eIF2 $\alpha$ and splicing of XBP-1 mRNA. As a model of acute starvation, culture medium of $\mathrm{C} 2 \mathrm{C} 12$ myotubes was replaced with sterile phosphate buffered saline (PBS) for $3 \mathrm{~h}$ or $6 \mathrm{~h}$ as described $(13,41)$. Protein extracts preprared from these myotubes were subjected to Western blotting. As shown in Figure $3.3 \mathrm{E}$, the phosphorylation of elF2 $\alpha$ was significantly increased both at $3 \mathrm{~h}$ and $6 \mathrm{~h}$ after incubation of myotubes with PBS. Similarly, protein levels of ATF3, ATF4, and CHOP and PDI were considerably increased in starved myotubes (Figure 3.3 E). Moreover, the levels of MyHCf protein were reduced indicating that $6 \mathrm{~h}$ of starvation was sufficient to cause significant atrophy in vitro (Figure $3.3 \mathrm{E}$ ). XBP-1 mRNA is induced by ATF6 and spliced by IRE1 in response to ER stress to produce a highly active transcription factor causing UPR induction (129). Therefore, we studied the splicing of XBP-1 mRNA in these cells by performing semi-quantitative reverse transcription PCR (RT-PCR) using primers that detect both unspliced and spliced mRNA. As shown in Figure 3.3 F, acute starvation of C2C12 myotubes by incubation with PBS dramatically increased the levels of spliced form of XBP-1 (sXBP-1) indicating that starvation also activates IRE1 arm of ER stress response in myotubes. 
Although ER stress has been studied in plethora of homeostasis-rectifying mechanisms, its involvement in skeletal muscle atrophy has not been explored. To understand the potential role of ER stress in the induction of atrophic program, we studied the effects of pharmacological inducers of ER stress on the expression of various components of UPS and ALS. $\mathrm{C} 2 \mathrm{C} 12$ myotubes were treated with ER stressor tunicamycin or thapsigargin for $18 \mathrm{~h}$ followed by measurement of mRNA of various genes by QRT-PCR assay. As shown in Figure $3.3 \mathrm{G}$, the expression of $M A F B x$ and MURF1 (important components of UPS) and LC3B and Beclinl (important components of ALS) was significantly increased in tunicamycin or thapsigargin treated myotubes compared to those treated with vehicle alone. Furthermore, mRNA levels of myosin heavy chain II (i.e. MYH4) were found to be significantly reduced in $\mathrm{C} 2 \mathrm{C} 12$ myotubes upon treatment with tunicamycin or thapsigargin (Figure $3.3 \mathrm{H}$ ). The increased expression of $\mathrm{CHOP}, \mathrm{ATF} 4$, and GADD34 in tunicamycin or thapsigargin treated $\mathrm{C} 2 \mathrm{C} 12$ myotubes confirmed that these agents activated UPR (Figure 3.3 I). Collectively, these results suggest that ER stress responsive pathways could be involved in the induction of muscle atrophy both through accelerating proteolysis and inhibiting expression of specific muscle genes such as MYH4.

\subsubsection{TRAF6 is Involved in the Increased Expression of Fn14 in Skeletal Muscle upon Starvation}

The TWEAK-Fn14 system has now emerged as one of the important regulators of skeletal muscle atrophy especially in disuse conditions (53). However, activation of TWEAK-Fn14 system does not occur in all atrophy conditions (53). We first investigated 
whether fasting affects the expression of TWEAK or its receptor Fn14 in skeletal muscle. The expression of Fn14 (but not TWEAK itself) was dramatically induced in skeletal muscle in response to fasting. Intriguingly, fasting-induced expression of Fn14 was significantly inhibited in skeletal muscle of TRAF6 ${ }^{\mathrm{mko}}$ mice compared to $\mathrm{TRAF}^{\mathrm{f} / \mathrm{f}}$ mice (Figure $3.4 \mathrm{~A}$ ). Western blotting using muscle extracts also validated that the expression of Fn14 is increased in skeletal muscle in response to fasting and TRAF6 is required for fasting-induced expression of Fn14 in skeletal muscle (Figure 3.4 B). Fasting did not affect the expression of IL-1 receptor (IL-1R), TNF receptor (TNFR) I or TNFRII in skeletal muscle of either TRAF6 $6^{\mathrm{f} / \mathrm{f}}$ or TRAF $6^{\mathrm{mko}}$ mice (our unpublished observation). These results suggest that the conditions of fasting specifically augment the expression of Fn14 receptor through TRAF6-dependent mechanisms.

While it was interesting to learn that TRAF6 regulates the expression of Fn14 in skeletal muscle, it was not clear whether TWEAK-Fn14 system also contributes to muscle atrophy upon starvation. To answer this question, we employed TWEAK-KO mice which have been previously found to be resistant to muscle atrophy in response to denervation (53). Three months old wild-type and TWEAK-KO mice were provided normal food or fasted for $24 \mathrm{~h}$ followed by euthanizing the mice and isolation of hind limb muscles for analyses. Transverse sections prepared from TA and soleus muscles were subjected to H\&E staining and the average fiber cross-sectional area (CSA) was quantified using morphometric methods. Interestingly, starvation-induced loss in fiber CSA was significantly inhibited in TA (Figure $3.4 \mathrm{C}$ ) and soleus (Figure $3.4 \mathrm{D}$ ) of TWEAK-KO mice compared to wild-type mice. 
To evaluate the mechanisms, we also studied whether the increased activity of TWEAK augments the expression of the components of UPS, ALS, and/or UPR in skeletal muscle. The activation of UPS was evaluated by measuring mRNA levels for $M A F B x$ and $M u R F 1$ by QRT-PCR. As shown in Figure 3.4 E, the starvation-induced increase in MuRF1 was significantly inhibited in GA muscle of TWEAK-KO mice compared to wild-type mice. In contrast, there was no significant difference in mRNA levels of $M A F B x$ between wild-type and TWEAK-KO mice upon starvation (Figure 3.4 E). QRT-PCR analysis also revealed that there was no significant difference in mRNA levels of LC3B, Beclin1, and Atg12 in skeletal muscle of wild-type and TWEAK-KO mice in response to starvation (Figure 3.4 F). Furthermore, there was also no significant difference in the starvation-induced expression of ER stress-related genes ATF4, GADD34, CHOP, HERPES, PDI, and GRP94 in fasted muscle of wild-type and TWEAK-KO mice (Figure $3.4 \mathrm{G}$ ). Taken together, these results indicate that TWEAKFn14 might be contributing to starvation-induced muscle loss through the activation of the components of UPS but not ALS or UPR pathway.

\subsubsection{E3 ubiquitin ligase activity of TRAF6 is essential for starvation-induced muscle atrophy}

TRAF6 functions both as an adaptor protein and E3 ubiquitin ligase catalyzing K63-linked autoubiquitination as well as ubiquitination of target proteins which stimulates protein trafficking (39). While E3 ubiquitin ligase activity of TRAF6 has been found to be critical for many of its cellular function, it has also been reported that ubiquitin ligase activity of TRAF6 may be dispensable for the activation of several 
downstream signaling pathways in response to specific stimuli (130). We first studied whether starvation increases TRAF6 autoubiquitination in skeletal muscle in vivo. Protein extracts prepared from skeletal muscle of unstarved or starved muscle of wild-type mice were immunoprecipitated with anti-TRAF6 followed by immunoblotting with anti-K63 ubiquitin which recognizes only K63-linked ubiquitinated proteins. As shown in Figure 3.5 A, TRAF6 ubiquitination was significantly increased in fasted skeletal muscle. Mutation of cystine to alanine (C70A) residue in TRAF6 zinc-finger domain renders it inactive to interact and bind with Ubc13-Uev complex (39). TRAF6C70A mutant has previously been used to study the role of TRAF6 E3 ubiquitin ligase activity in various cellular responses $(30,39)$. To understand whether E3 ubiquitin ligase activity of TRAF6 is required for fasting-induced muscle atrophy, TA muscle of wild-type mice were electroporated with vector alone or with TRAF6C70A cDNA. Efficiency of gene delivery was monitored by co-electroporation with a green fluorescence protein (GFP) vector as described (131). After 10 days of plasmid electroporation, the mice were given normal food or fasted for $24 \mathrm{~h}$ and TA muscle isolated were used to make sections and measurement of fiber CSA. Interestingly, overexpression of TRAF6C70A mutant significantly inhibited starvation-induced fiber atrophy in TA muscle of mice (Figure 3.5 $\mathrm{B}$ and $3.5 \mathrm{C})$.

To further evaluate the role of ubiquitin ligase activity of TRAF6 in starvationinduced muscle atrophy, we investigated whether overexpression of TRAF6C70A mutant can affect the activation of atrophic program in cultured myotubes. $\mathrm{C} 2 \mathrm{C} 12$ myoblasts were stably transfected with vector alone or TRAF6C70A followed by their differentiation into myotubes. To induce starvation, the myotubes were incubated in PBS 
for different length of time. As shown in Figure 3.5 D, overexpression of TRAF6C70A inhibited the starvation-induced atrophy in cultured myotubes. Quantitative estimation of myotube diameter also revealed that TRAF6C70A mutant inhibits atrophy in cultured myotubes (Figure 3.5 E and 3.5 F). Finally, we investigated whether overexpression of TRAF6C70A mutant can block the expression of the markers of UPS, ALS, and ER stress response in myotubes. Interestingly, fasting-induced increase in mRNA levels of MAFBx, MuRF1, LC3B, and CHOP was found to be significantly inhibited in TRAF6C70A transfected myotubes compared to those transfected with vector alone (Figure $3.5 \mathrm{G}$ ). Together, these experiments provide convincing evidence that E3 ubiquitin ligase activity of TRAF6 is essential for starvation-induced skeletal muscle atrophy. 


\subsection{CONCLUSION AND DISCUSSION}

This study identifies TRAF6 as a novel regulator of a complex array of intracellular mechanisms that underlie the starvation-induced atrophic response in skeletal muscle. This investigation is also first one to recognize potential roles of the TWEAK-Fn14 system and UPR pathways in development of skeletal muscle atrophy in response to starvation.

It has been consistently observed that in almost all atrophic conditions, the activation of UPS and ALS is a common denominator for the loss of skeletal muscle mass. Rapid muscle atrophy might stem from degradation of thick and thin filament proteins some of which are targeted by muscle-specific E3 ubiquitin ligases MuRFI and $\operatorname{MAFBx}(9,27,105,108)$. As also noticed in other atrophic conditions (117), one of the mechanisms by which TRAF6 mediates starvation-induced muscle atrophy is through the activation of UPS because fasting-induced overall protein ubiquitination and the expression of both $M A F B x$ and $M u R F 1$ were significantly reduced in skeletal muscle of $\mathrm{TRAF}^{\mathrm{mko}}$ mice compared to control littermates (Figure 3.2 A, 3.2 B).

The contribution of ALS has gained increasing attention in regulation of skeletal muscle mass in both physiological and pathological conditions. It has also been found that along with UPS, the activation of ALS also induces myofiber degradation in atrophying skeletal muscle $(13,18,132)$. Although autophagy is a homeostasis maintenance mechanism and physiological autophagy is necessary for removal of protein aggregates and defunct cellular compartments $(15,18)$, hyper activated ALS can contribute to muscle proteolysis in various conditions including fasting (18). Consistent with published reports $(13,14)$, our results indicate that fasting causes increased 
expression of several autophagy genes (Figure $3.2 \mathrm{C}, 3.2 \mathrm{D}$ ), activation of FOXO3a transcription factor (Figure 3.2 E) and AMPK (Figure 3.2 F). While AMPK is known to stimulate expression of $\mathrm{LC} 3 \mathrm{~B}, \mathrm{FOXO} 3 \mathrm{a}$ transcription factor is purported to upregulate $M A F B x$ and $M u R F 1$ and regulates ALS in skeletal muscle $(13,14)$. All these markers and effectors of macroautophagy were found to be significantly inhibited in TRAF ${ }^{\text {mko }}$ mice compared to $\mathrm{TRAF}^{\mathrm{f} / \mathrm{f}}$ mice providing another evidence about the role of TRAF6 and autophagy in starvation-induced atrophy. TRAF6 interacts with LC3B through p62 (48) and ubiquitinylates (Lys63-linked) Beclin-1 and thus further regulates autophagosome formation in response to toll-like receptor 4 (TLR4) signaling (93). Our results also indicate similar interaction in starvation-induced muscle atrophy because levels of $p 62$, LC3B and Beclin-1 were found to be induced by fasting in TRAF6 ${ }^{\mathrm{fff}}$ mice and were rescued in TRAF6 ${ }^{\mathrm{mko}}$ mice (Figure $3.2 \mathrm{C}, 3.2 \mathrm{D}$ ). Though it has been suggested that proteasomal degradation dominates over lysosomal breakdown of proteins during muscle atrophy $(13,14)$, our results indicate that irrespective of their relative contribution, TRAF6 recruits both UPS and ALS in starvation-induced muscle atrophy.

ER is a site for and an important regulator of protein folding, trafficking, targeting, and quality control. In the conditions of increased unfolded protein load/stress, ER stimulates an elaborate corrective response known as UPR (133) that is mediated by three ER membrane-associated proteins, PERK, IRE1, and ATF6. UPR pathways have been found to be involved in several pathological and metabolic disorders such as impaired glucose metabolism $(134,135)$, adipocyte stress $(136,137)$ and inflammatory response and metabolic abnormalities (138-140). However, there has been no published evidence yet suggesting direct involvement of UPR in skeletal muscle atrophy. In an 
attempt to study involvement of ER stress in disuse atrophy, it was previously reported that while the genes that maintain sarcoplamic reticulum (SR) calcium levels are induced in disuse atrophy, there is no such increase in the markers of ER stress or UPR (141). However, a recent report suggests that in response to starvation, the expression of ATF4 is increased which promotes myofiber atrophy (23). Interestingly, it was found that ATF4 affects the expression of a few genes involved in muscle growth, however, it did not affect the expression of $M A F B x$ or $M u R F 1$ suggesting that ATF4 can induce muscle atrophy through mechanisms independent of activation of UPS (23). Although ATF4 can be activated by multiple mechanisms in fasting conditions, it belongs to PERK/eIF2 $\alpha$ branch of UPR and thus there is a possibility that UPR pathways are activated and mediate starvation-induced muscle atrophy.

Recent investigations have highlighted the role ER stress and UPR pathways play in energy and glucose metabolism and have shown ER sensitivity towards glucose availability. Transcriptional networks activated by ER stress regulate the expression of several genes involved in glucose metabolism. GADD34, XBP-1 and ATF6 have been found to be involved in glucose output, glycogen synthesis and gluconeogenesis (135, 142-144) implicating all the three arms of UPR play role in energy and glucose metabolism. Muscles being the largest reservoir of proteins are the first ones to be mobilized as a source of amino acids for gluconeogenesis. Although UPR activation and its role in glucose metabolism in atrophying muscle have not been yet elucidated, there is a possibility that fasting activates UPR which in turn induces secondary mechanisms regulating muscle atrophy. Our results provide initial evidence that UPR is activated in skeletal muscle both in vitro and in vivo (Figure 3.3) and underline a possibility that 
through transcriptional activation of downstream effectors, UPR pathways could be instrumental in regulation of skeletal muscle mass. This inference is also supported by our findings that treatment of myotubes with ER stressor tunicamycin or thapsigargin augmented the expression of components of UPS and ALS (Figure $3.3 \mathrm{G}$ ). Interestingly all the markers of UPR were significantly inhibited in $\mathrm{TRAF}^{\mathrm{mko}}$ mice (Figure 3.3) suggesting that TRAF6 plays a critical role in induction of UPR in starvation-induced skeletal muscle atrophy. Previously published reports bolster this observation by implicating TRAF2 in activation of UPR and proteolytic degradation and cell death (145). TRAF2 is another E3 ubiquitin ligase of TRAF family and shares many common features with TRAF6 (116). Therefore, it is possible that functional redundancy exists between TRAF2 and TRAF6 in the activation of UPR pathways in response to different stimuli. Future research will reveal an interaction and/or mechanisms through which TRAF6 regulates ER stress response in fasted muscle.

TWEAK-Fn14 axis is another recently discovered regulator of skeletal muscle atrophy $(53,99)$. Increased expression of Fn14 receptor appears to be a key determinant in TWEAK-mediated skeletal muscle wasting especially in disuse conditions (53). In the present study, we observed that the levels of Fn14 are dramatically induced in fasted skeletal muscle and that the TWEAK-Fn14 system contributes to the starvation-induced muscle atrophy in mice (Figure 3.4). It is noteworthy that although Fn14 is a cell surface receptor and TRAF6 is an intracellular protein, signaling through TRAF6 induces the expression of Fn14 in fasted muscles. Our previous investigations showed that TRAF6 does not play a role in the inducible expression of Fn14 in denervated muscle (117) further suggesting that TRAF6 mediates different types of muscle atrophy through 
activation of distinct mechanisms. The observation that Fn14 is one of the downstream targets of TRAF6 in starvation-induced atrophy is also supported by our data that inhibition of TWEAK-Fn14 system did not affect the activation of either ALS (Figure 3.4 F) or UPR (Figure $3.5 \mathrm{G}$ ) in skeletal muscle whereas ablation of TRAF6 inhibited both these pathways in response to starvation (Figure 3.2).

In the recent years, significant progress has been made to understanding the mechanisms by which TRAF6 propagates downstream signaling. The most accepted model suggests that upon activation, the RING finger ubiquitin E3 ligase domain makes complexes with E2 conjugating enzyme Ubc13/Uev1a to mediate conjugation of K63linked ubiquitin chains to TRAF6 substrates, including TRAF6 itself. These chains recruit several factors including adaptor proteins $\mathrm{TAB} 2 / 3$, which contain atypical zinc finger domains with affinity for binding K63-linked ubiquitin chains, resulting in the activation of transforming growth factor- $\beta$ activated kinase 1 (TAK1) complex. However, Walsh et al have previously reported that while the RING finger domain of TRAF6 is essential for the activation of TAK1, it was not required for interaction between TRAF6 and the TAK1 complex in vitro (130). Furthermore, this study suggested that TRAF6 autoubiquitination was dispensable for both its interaction with and activation of the TAK1 complex and also for RANKL-induced osteoclastogenesis (130). In contrast, our experiments demonstrate that TRAF6 undergoes K63-linked autoubiquitination in skeletal muscle in response to starvation (Figure $3.5 \mathrm{~A}$ ). Furthermore, using TRAF6 mutant lacking ubiquitin ligase activity (i.e. TRAF6C70A) (39), we found that the ligase activity of TRAF6 is essential for orchestrating the activation of ALS, UPS, and UPR upon acute starvation (Figure $3.5 \mathrm{G}$ ). The requirement of E3 ubiquitin ligase for muscle 
atrophy in response to starvation (Figure 3.5) but not during osteoclastogenesis (130) suggests that TRAF6 induces activation of different signaling complexes in a contextdependent manner. It is also notable that starvation-induced muscle atrophy may not even involve the activation of TAK1-IKK-NF- $\mathrm{KB}$ pathway for which the autoubiquitination of TRAF6 or its E3 ligase activity has been found dispensable during osteoclastogenesis (130).

In conclusion, the results of present study identify TRAF6 as a novel regulator of starvation-induced atrophy. Since molecular pathways regulated by TRAF6 are also implicated in many other cell types and physiological responses, a better understanding of its regulatory role will be of significant clinical importance for developing new therapeutic strategies for treatment of muscle disorders and other diseases. 

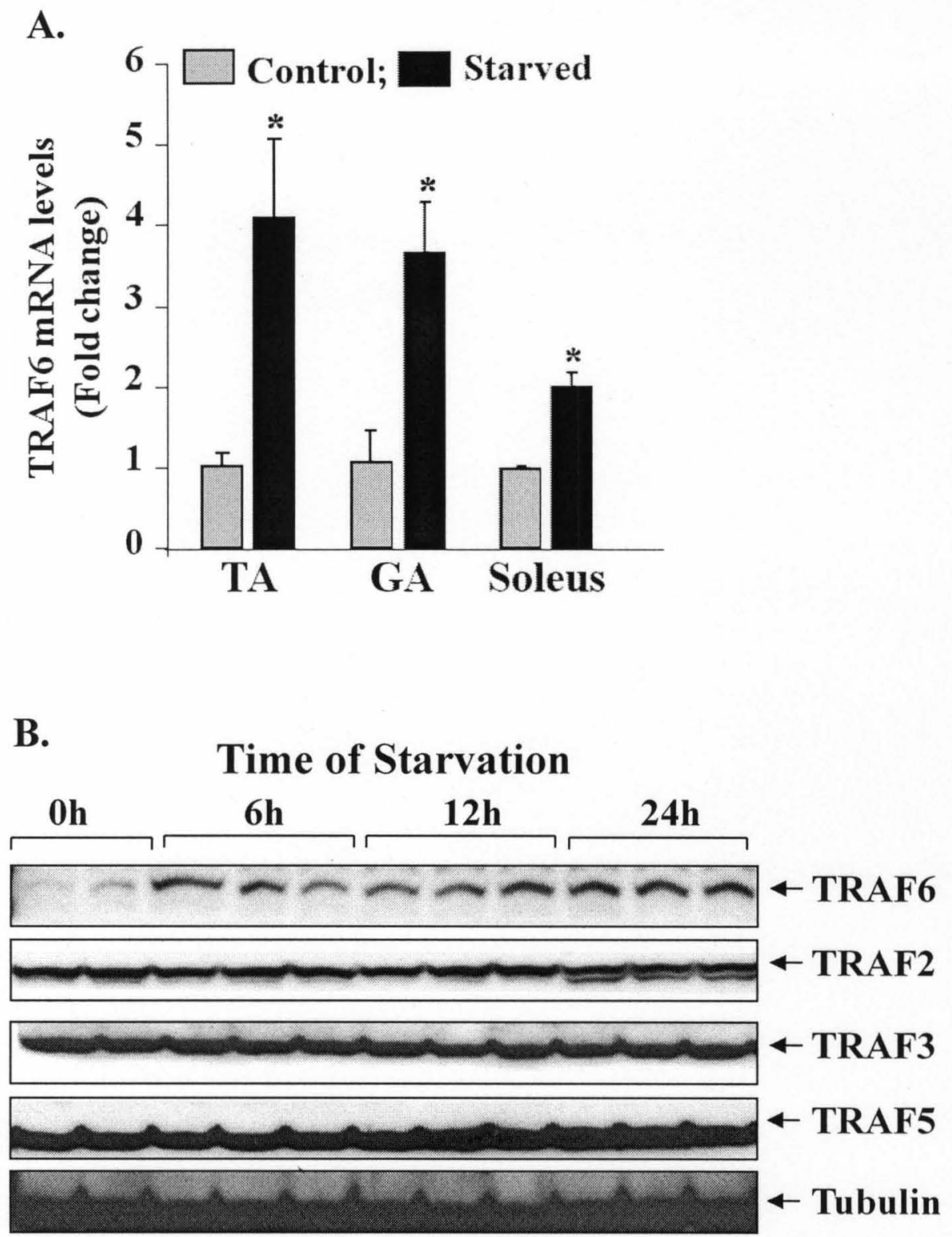
C.

Time of Starvation
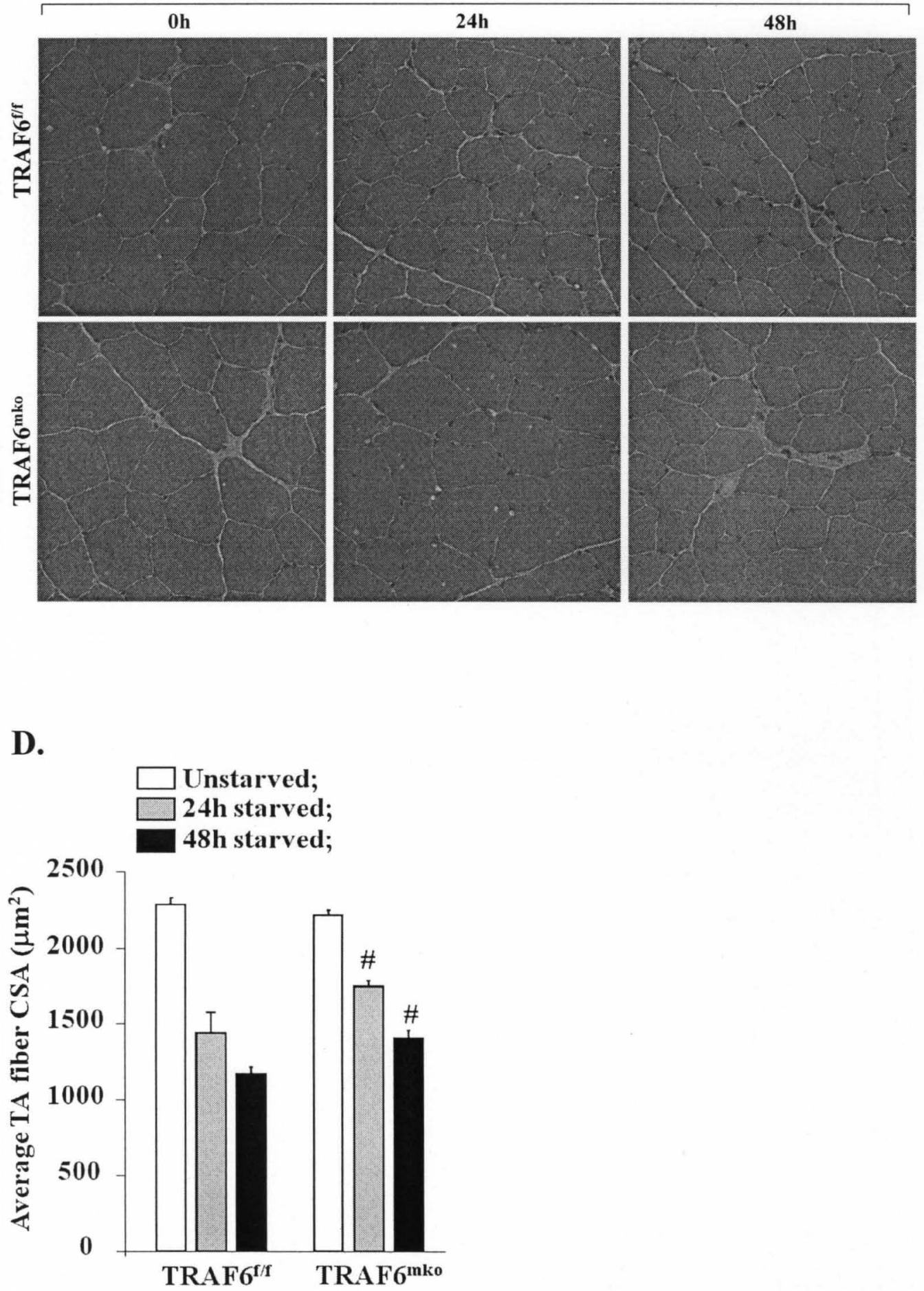
E.

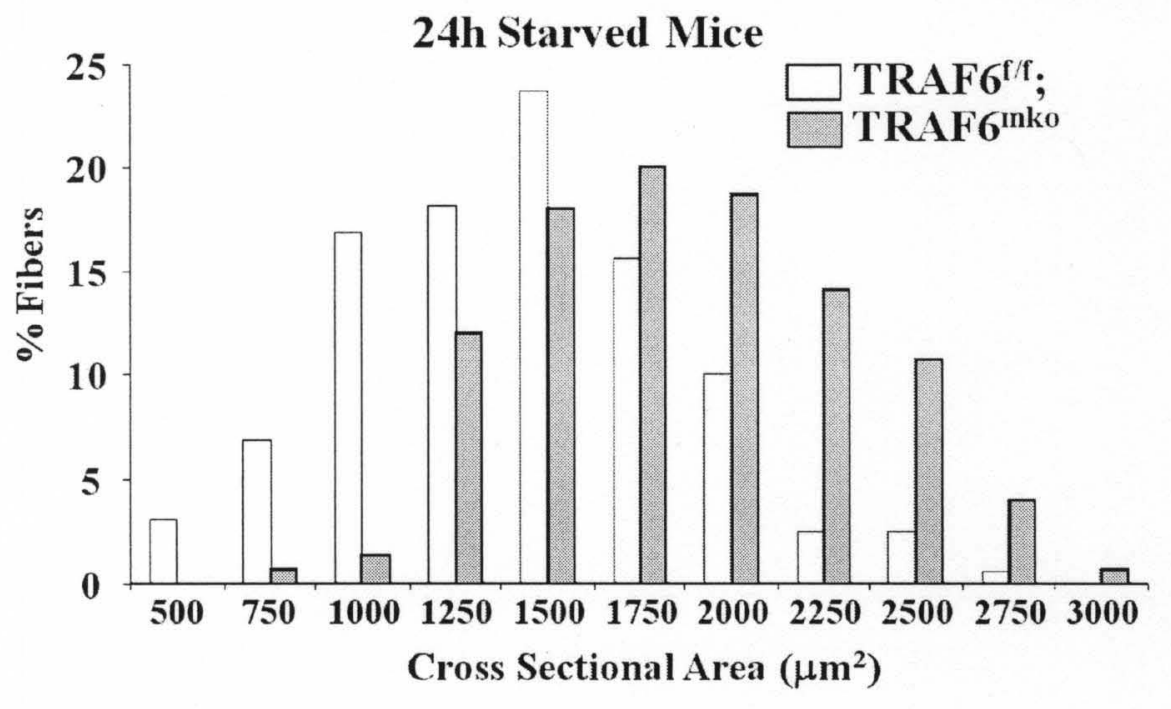

F.

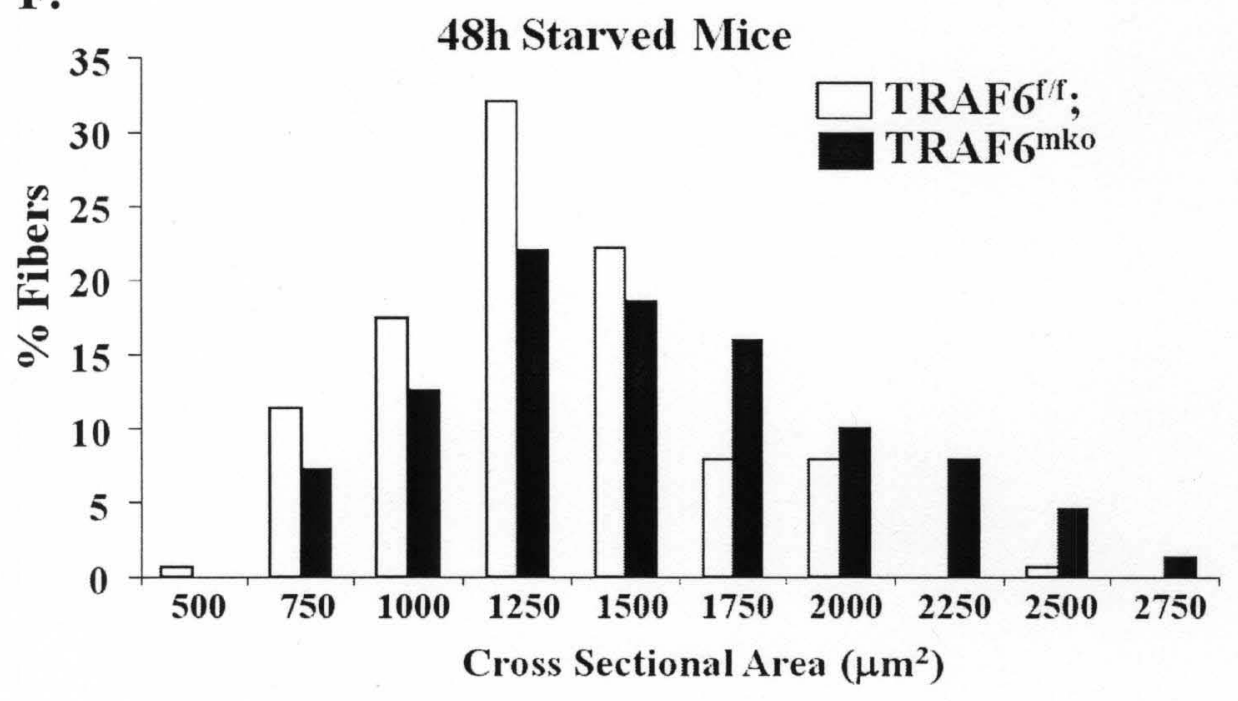




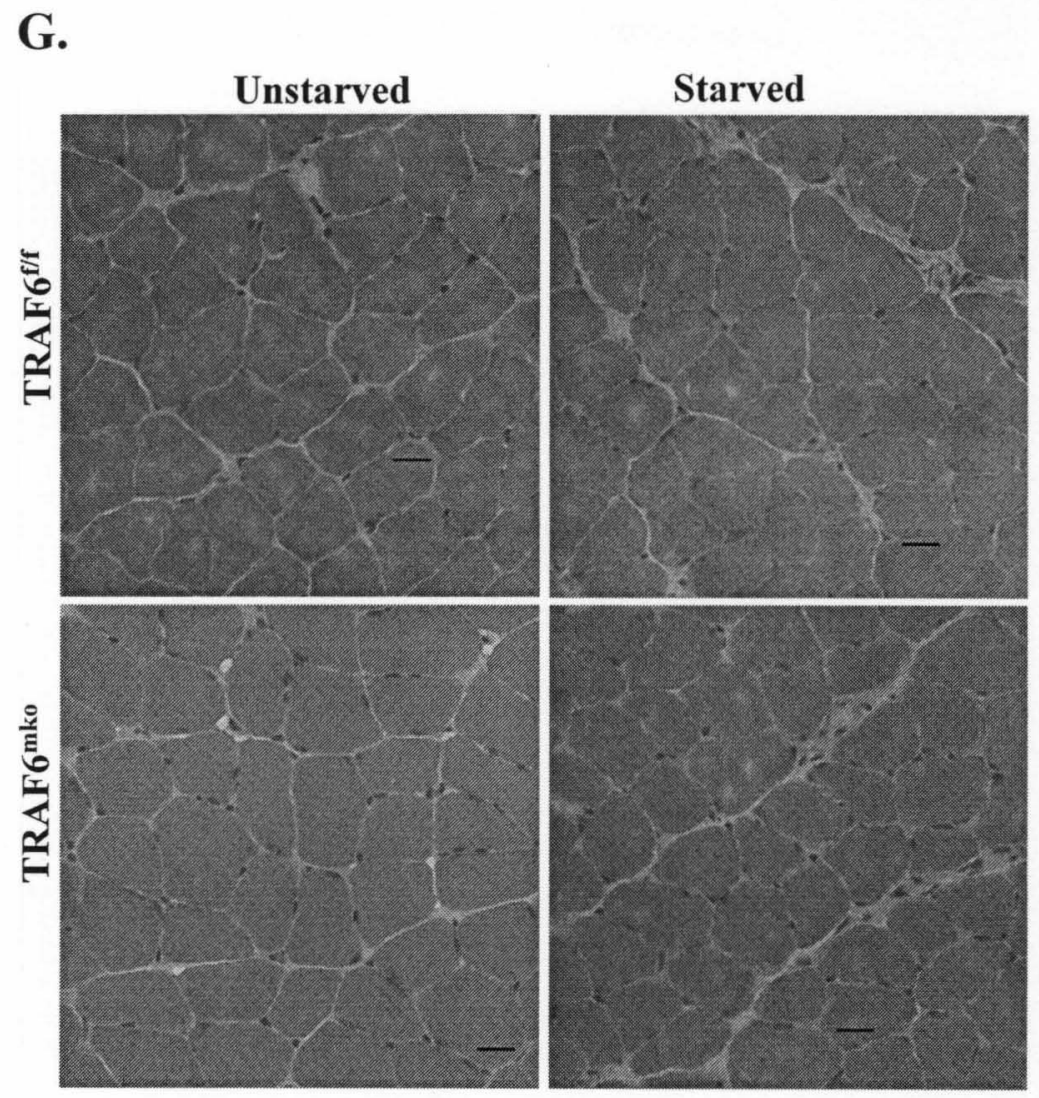

H.

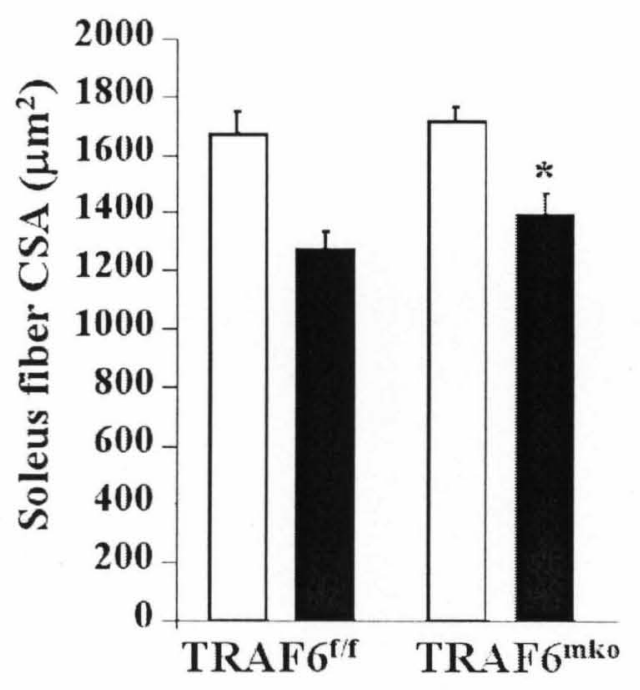


Figure 3.1. Involvement of TRAF6 in fasting-induced muscle atrophy. (A) Transcript levels of TRAF6 in tibial anterior (TA), gastrocnemius (GA), and soleus muscle control and $24 \mathrm{~h}$ fasted wild-type mice. Error bars represent SD. $\mathrm{N}-=3$ in each group. ${ }^{*} \mathrm{p}<0.01$, values significantly different from unstarved muscle. (B) Western blot analyses of TA muscle extracts for TRAF2, TRAF3, TRAF5, TRAF6 proteins at different time points after food deprivation. (C) 12-week-old TRAF6 ${ }^{\mathrm{f} / \mathrm{f}}$ and TRAF $6^{\mathrm{mko}}$ mice were starved for $24 \mathrm{~h}$ or $48 \mathrm{~h}$. Representative photomicrographs of H\&E-stained sections of TA muscle of $\mathrm{TRAF}^{\mathrm{f} / \mathrm{f}}$ and TRAF6 ${ }^{\mathrm{mko}}$ mice $24 \mathrm{~h}$ and $48 \mathrm{~h}$ after starvation. (D) mean CSA and (E and F) Frequency distribution histograms representing cross-sectional area (CSA) of fibers in TA muscle from TRAF6 ${ }^{\mathrm{f} / \mathrm{f}}$ (open bars) and TRAF $6^{\text {mko }}$ (shaded bars) at $24 \mathrm{~h}$ and $48 \mathrm{~h}$ after starvation. 12-week-old TRAF6 $^{\mathrm{f} / \mathrm{f}}$ and $\mathrm{TRAF}^{\mathrm{mko}}$ mice were starved for $24 \mathrm{~h}$. (G) Representative photomicrographs of H\&E-stained sections of soleus muscle sections of TRAF $6^{\mathrm{f} / \mathrm{f}}$ and TRAF $6^{\mathrm{mko}}$ mice $24 \mathrm{~h}$ after starvation. (H) Average fiber cross-sectional area (CSA) in soleus muscle of control and $24 \mathrm{~h}$ starved TRAF6 ${ }^{\mathrm{tf} / \mathrm{f}}$ and TRAF6 ${ }^{\mathrm{mko}}$ mice. $\left(\mathrm{N}=8\right.$ in each group) Error bars represent $\mathrm{SD}$. ${ }^{*} \mathrm{p}<0.05$, values significantly different from fasted TRAF6 ${ }^{\mathrm{f} / \mathrm{f}}$ mice Scale bar: $20 \mu \mathrm{m}$. 
A.

\begin{tabular}{|llllllll}
\multicolumn{4}{c}{ TRAF $^{\mathrm{f} / \mathrm{f}}$} & & \multicolumn{4}{c}{ TRAF $^{\text {mko }}$} \\
& $\mathrm{C}$ & $\mathrm{S}$ & $\mathrm{S}$ & $\mathrm{C}$ & $\mathrm{C}$ & $\mathrm{S}$ & $\mathrm{S}$
\end{tabular}
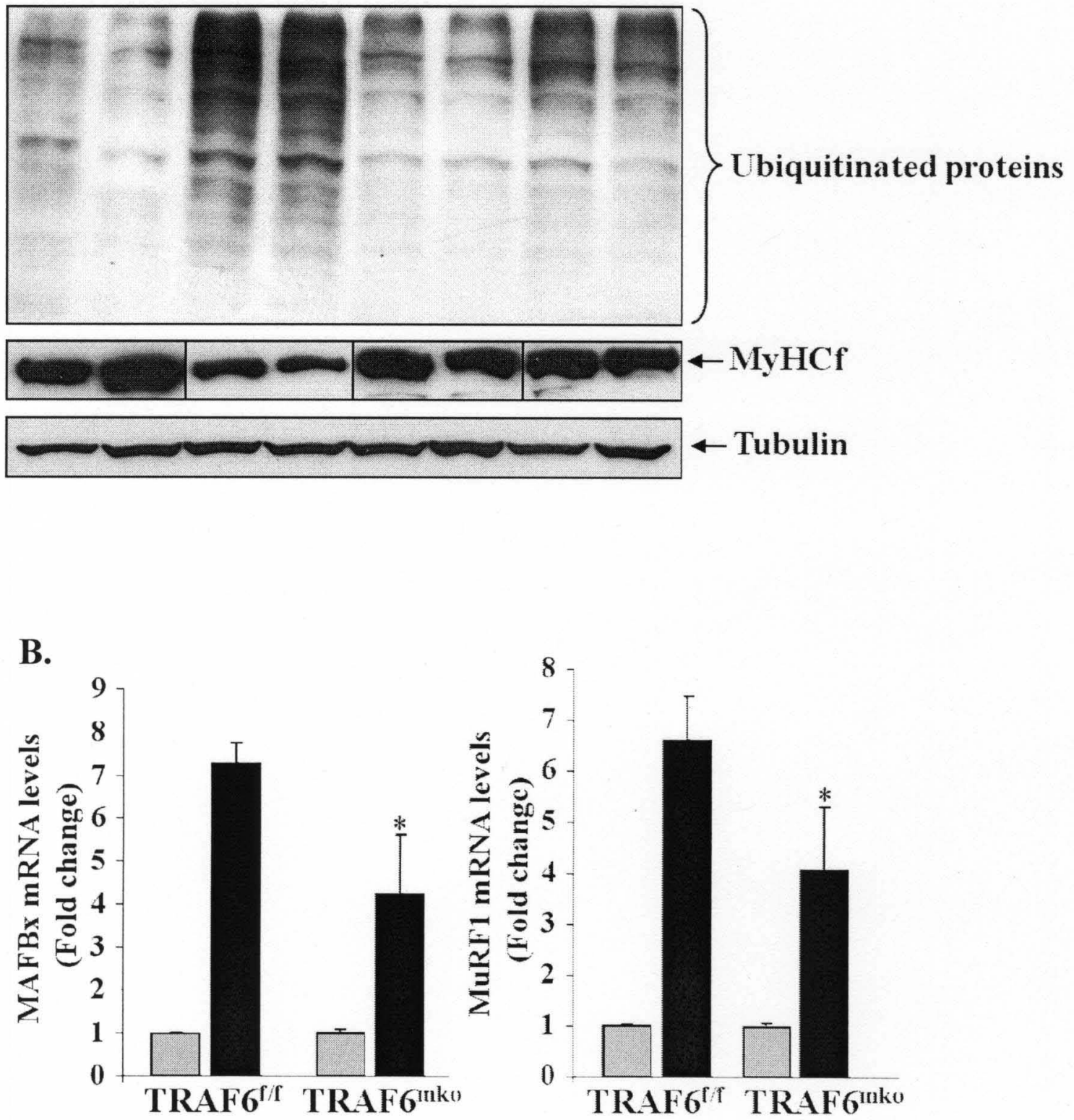
C.

$\square$ Unstarved; $\square$ Starved
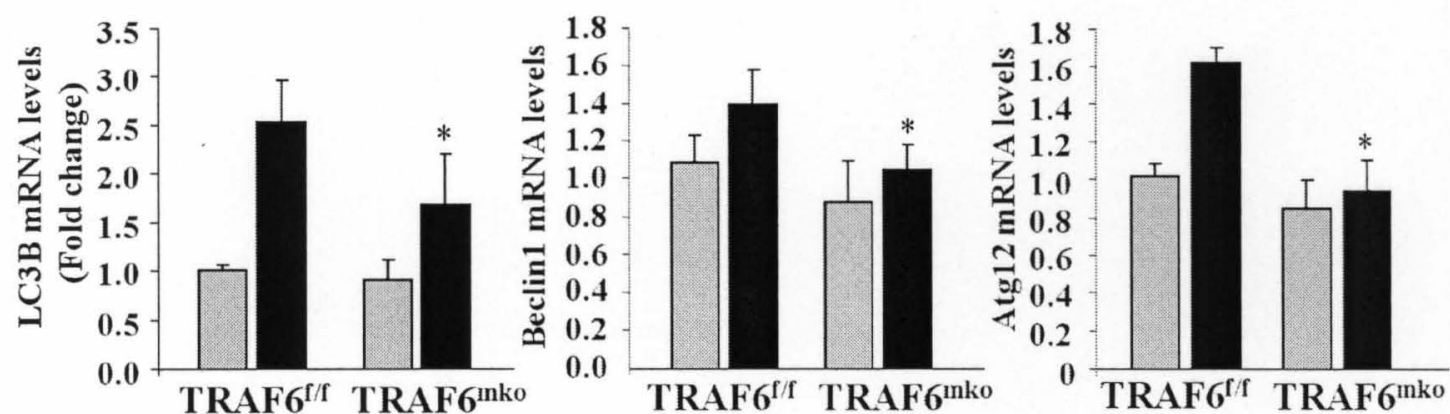

D.

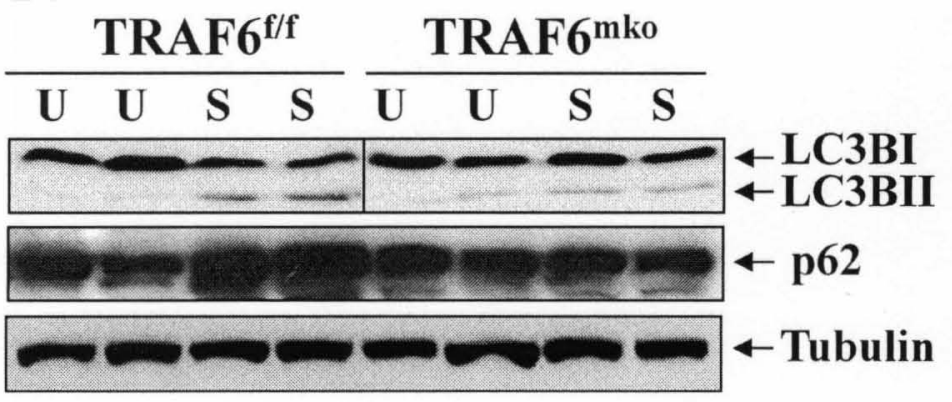

E.

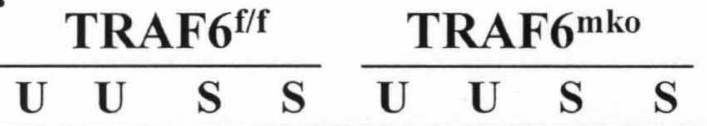

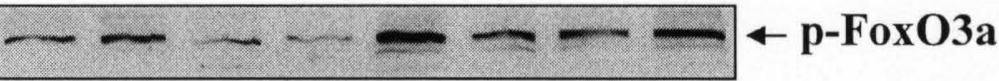

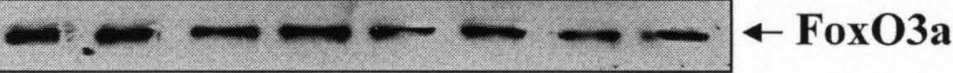

$$
\begin{aligned}
& -1-\infty-\infty-\infty \text { pAkt }
\end{aligned}
$$

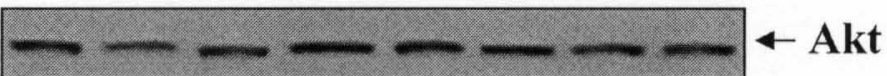

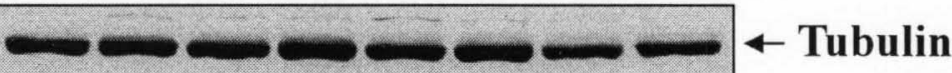




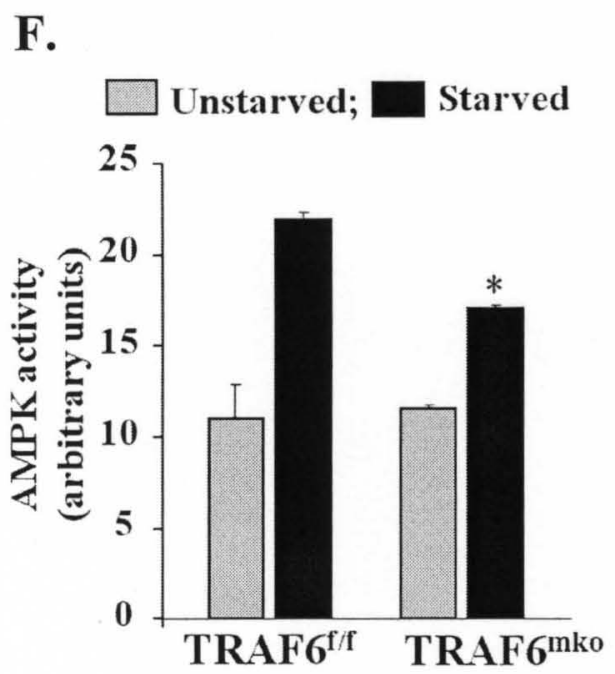

Figure 3.2: Role of TRAF6 in fasting-induced activation of ubiquitin-proteasome system and autophagy in skeletal muscle. $\mathrm{TRAF}^{\mathrm{f} / \mathrm{f}}$ and $\mathrm{TRAF} 6^{\mathrm{mko}}$ mice were starved for $24 \mathrm{~h}$ and GA or TA muscle isolated was analyzed. (A) Representative immunoblots showing levels of ubiquitinated proteins (top panel), MyHCf (middle panel), and unrelated protein tubulin (bottom panel) TA muscle. (B) Transcript levels of $M A F B x$ and MuRF1 measured by QRT-PCR assay were significantly lower in starved GA muscle of TRAF $6^{\mathrm{mko}}$ mice compared with TRAF6 ${ }^{\mathrm{f} / \mathrm{f}}$ mice. (C) Fold change in transcript levels of autophagy-related genes LC3B, Beclin1, and Atg12 in starved GA muscle of TRAF6 ${ }^{\text {mko }}$ mice compared with $\mathrm{TRAF}^{\mathrm{f} / \mathrm{f}}$ mice $(\mathrm{N}=4)$. (D) Immunoblots presented here demonstrate reduced protein levels of $\mathrm{p} 62$ and inhibition of conversion of LC3BI protein into LC3BII. (E). Levels of phosphorylation of FOXO3a and Akt in unstarved and 24h starved TA muscle of TRAF $6^{\mathrm{f} / \mathrm{f}}$ and TRAF $6^{\mathrm{mko}}$ mice. (F) Enzymatic activity of AMPK in TA muscle extracts of $\mathrm{TRAF}^{\mathrm{f} / \mathrm{f}}$ and $\mathrm{TRAF}{ }^{\mathrm{mko}}{ }$ mice. Error bars represent $\mathrm{SD}$. ${ }^{*} \mathrm{p}<0.05$, values significantly different from starved TA muscle of $\mathrm{TRAF}^{\mathrm{f} / \mathrm{f}}$ mice. 
A.

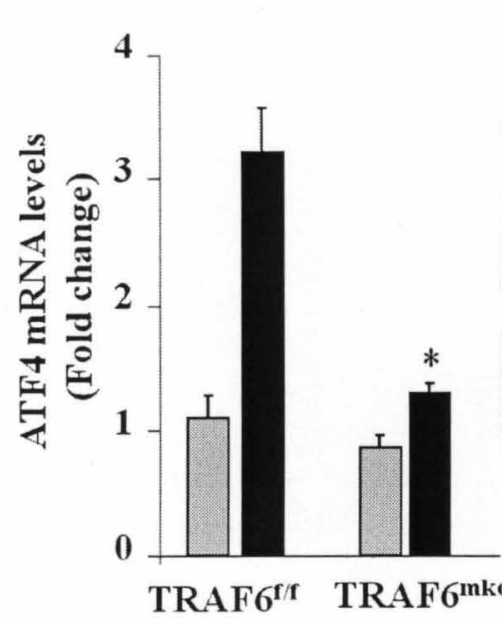

$\square$ Unstarved; $\square$ Starved
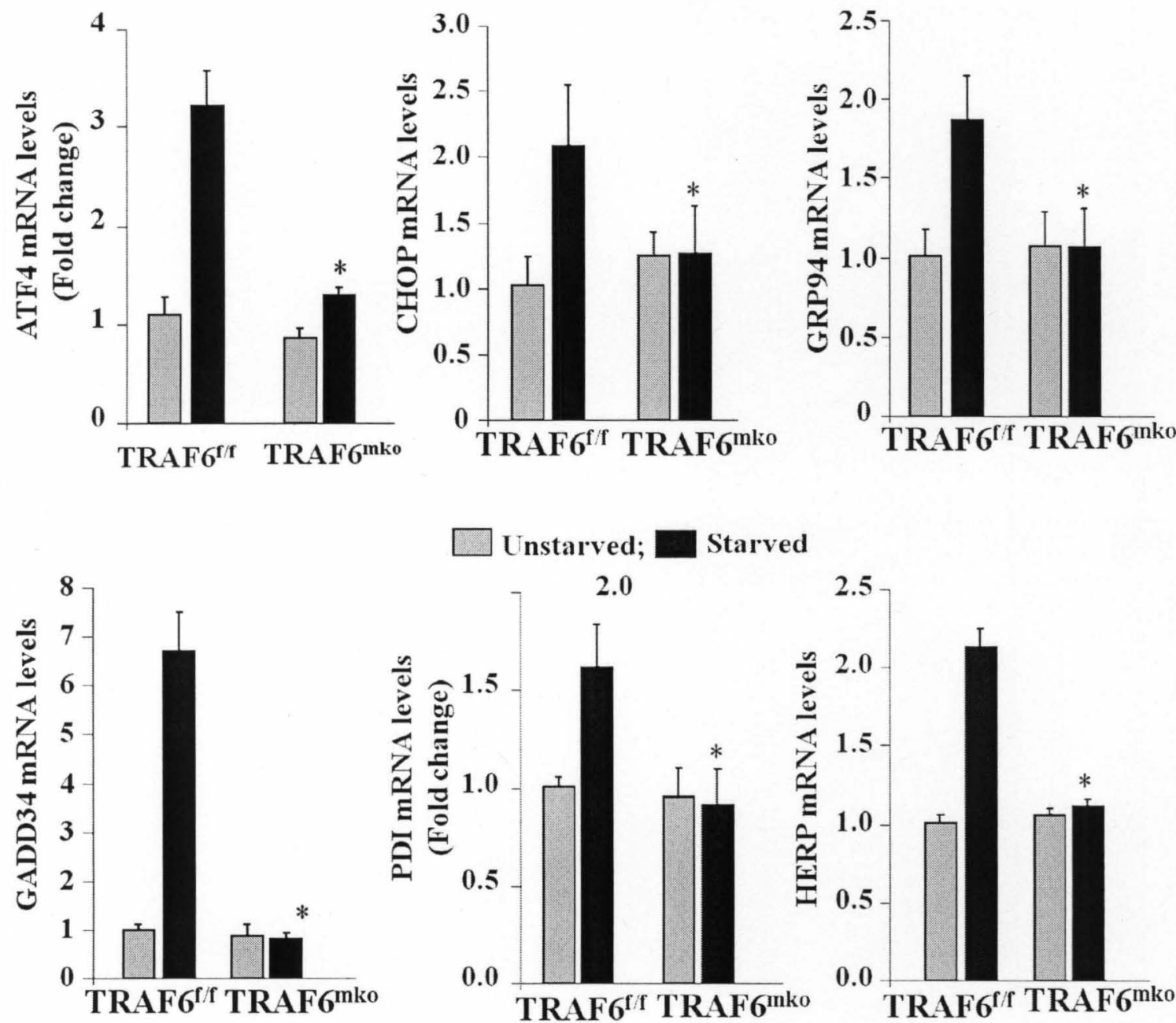

B.

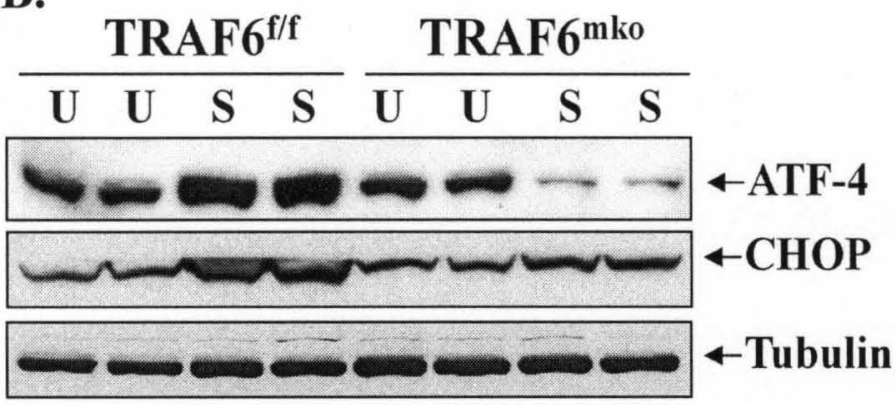



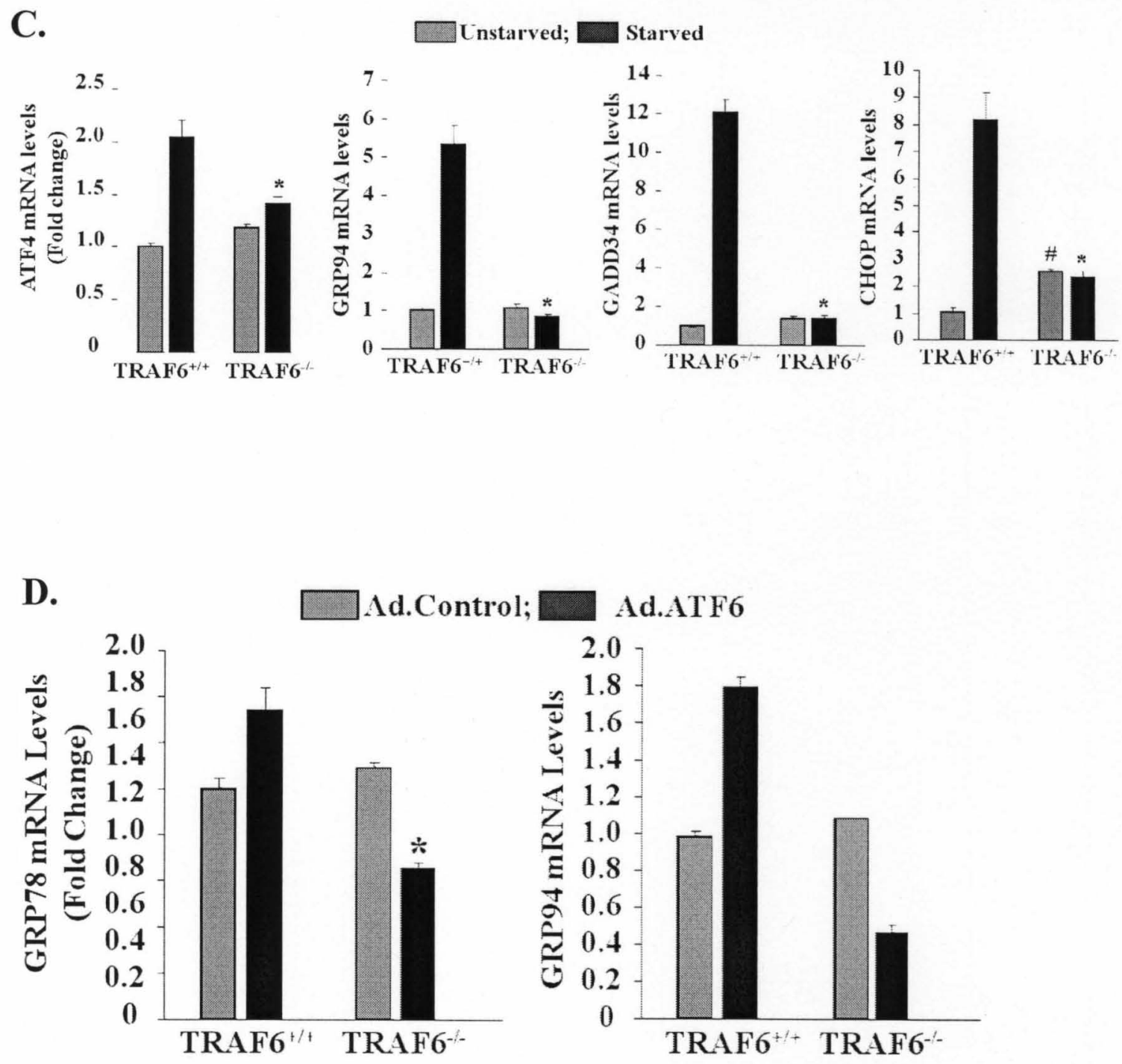
E.

Time of Starvation
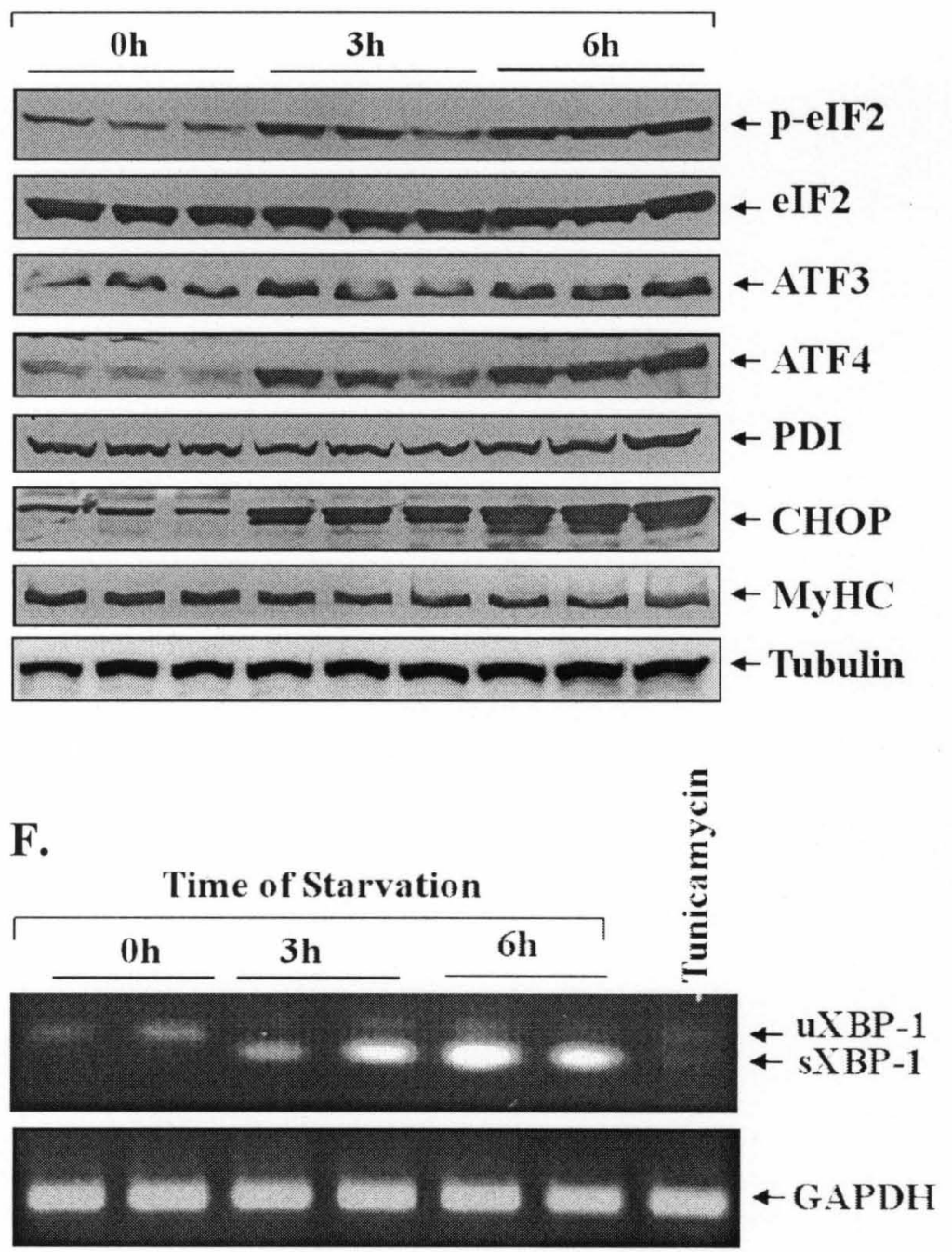

G.

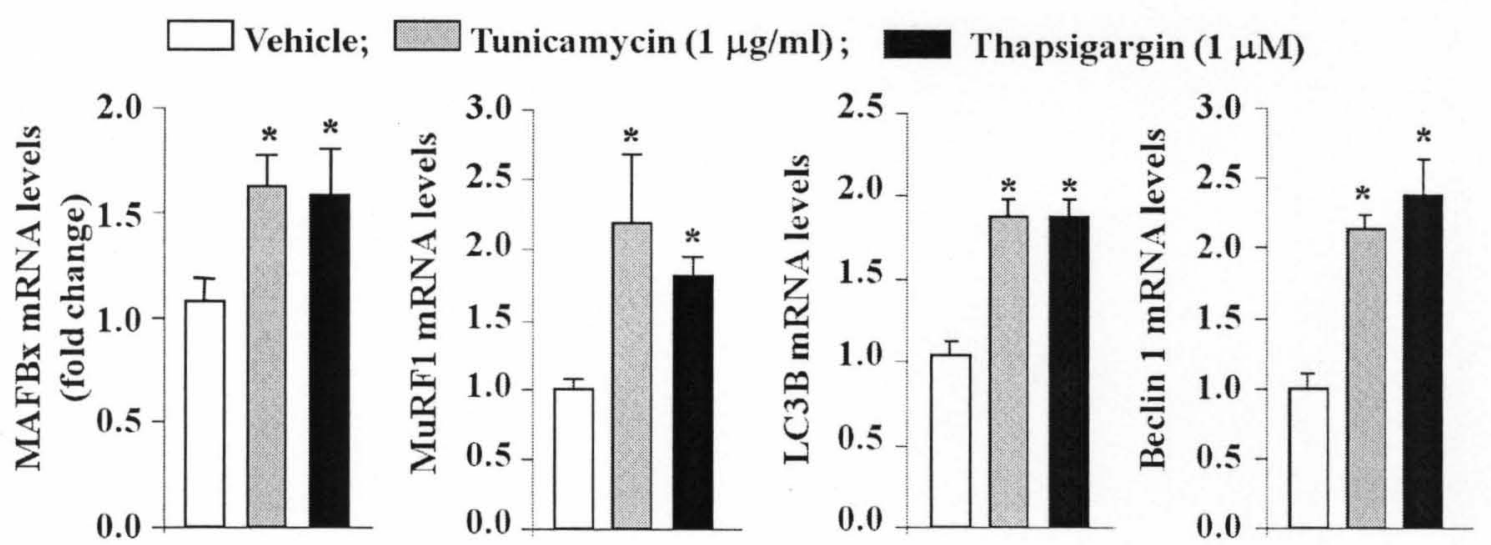


H.

I.
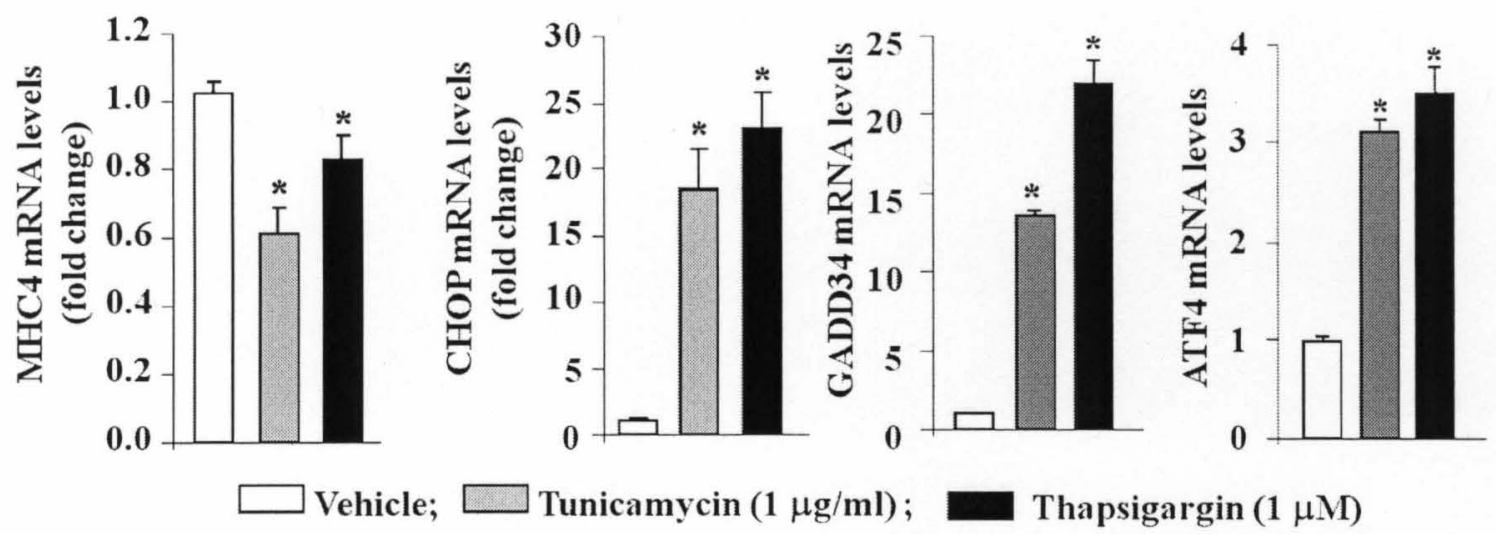

Tunicamycin $(1 \mu \mathrm{g} / \mathrm{ml})$;

Thapsigargin $(1 \mu \mathrm{M})$

Figure 3.3: Role of TRAF6 in the induction of ER stress responsive genes in skeletal muscle upon fasting. Three months old $\mathrm{TRAF} 6^{\mathrm{f} / \mathrm{f}}$ and $\mathrm{TRAF} 6^{\mathrm{mko}}$ mice were fasted for 24h and GA muscle isolated was used for biochemical analysis. (A) Transcript levels of ATF4, CHOP, GRP94, GADD34, and PDI were found to be significantly lower in fasted GA muscle of TRAF $6^{\text {mko }}$ mice compared with $\mathrm{TRAF}^{\mathrm{f} / \mathrm{f}}$ mice. $\mathrm{N}=4$ in each group. Error bars represent $\mathrm{SD}$. ${ }^{*} \mathrm{p}<0.05$, values significantly different from starved muscle of $\mathrm{TRAF}^{\mathrm{f} / \mathrm{f}}$ mice. (B) Representative immunoblots presented here demonstrate reduced protein levels of ATF4 and CHOP in TA muscle of starved TRAF $6^{\text {mko }}$ mice compared with $\mathrm{TRAF}^{\mathrm{f} / \mathrm{f}}$ mice. $\mathrm{C} 2 \mathrm{C} 12$ myoblasts were differentiated into myotubes and starved for 3 and 6 hrs in PBS. (C) Cultured TRAF6 ${ }^{+/+}$and TRAF6 ${ }^{-/-}$MEFs were incubated in serumfree medium for $24 \mathrm{~h}$ followed measurement of mRNA levels of ER stress responsive genes ATF4, GADD34, GRP94, and CHOP by QRT-PCR. (D) Cultured TRAF6 ${ }^{+/+}$and TRAF6 $^{-1-}$ MEF were transduced with ATF6 adenovirus (Ad.ATF6) or control (Ad.Control) for $24 \mathrm{~h}$ followed by measurement of mRNA levels of ER Chaperons GRP78 and GRP94. Error bars represent SD. ${ }^{*}$ p $<0.01$, values significantly different from serum-starved $\mathrm{TRAF}^{+/+} \mathrm{MEF}$. ${ }^{\#} \mathrm{p}<0.05$, values significantly different from unstarved TRAF6 $^{+/+}$MEF. ${ }^{*} \mathrm{p}<0.05$, values significantly different from Ad.ATF6-transduced TRAF6 $^{+/+}$MEF. (E) Representative immunoblots from two independent experiments performed in triplicates, presented here show increased phosphorylation of eIF2 $\alpha$ protein and elevated levels ER stress responsive proteins ATF3, ATF4, PDI and CHOP in $3 \mathrm{~h}$ or $6 \mathrm{~h}$ starved myotubes. Levels of MyHCf were reduced upon incubation of myotubes in PBS. (F) Splicing of XBP-1 increased upon fasting or ER stressor tunicamycin in $\mathrm{C} 2 \mathrm{C} 12$ myotubes measured by reverse transcription-PCR assays using primers that detected both unspliced and spliced form of XBP-1. $u X B P-1$, unspliced XBP-1; $s X B P-1$, spliced XBP1. Effect of ER stressor on expression of UPS and ALS genes in C2C12 myotubes. C2C12 myoblasts were differentiated into myotubes and ER stress was induced by 
treatment with tunicamycin or thapsigargin for $18 \mathrm{~h}$. (G) QRT-PCR analyses showed that treatment with tunicamycin or thapsigargin significantly increased transcript levels of MAFBx, MuRF1, LC3B, and Beclin1 in myotubes. (H). Treatment with tunicamycin or thapsigargin significantly reduced mRNA levels of MHC4 in $\mathrm{C} 2 \mathrm{C} 12$ myotubes. (I) Transcript levels of ER stress-related genes CHOP, GADD34 and ATF4 was increased upon treatment with tunicamycin or thapsigargin. Error bars represent SD. ${ }^{*} \mathrm{p}<0.05$, values significantly different from myotubes treated with vehicle alone. 

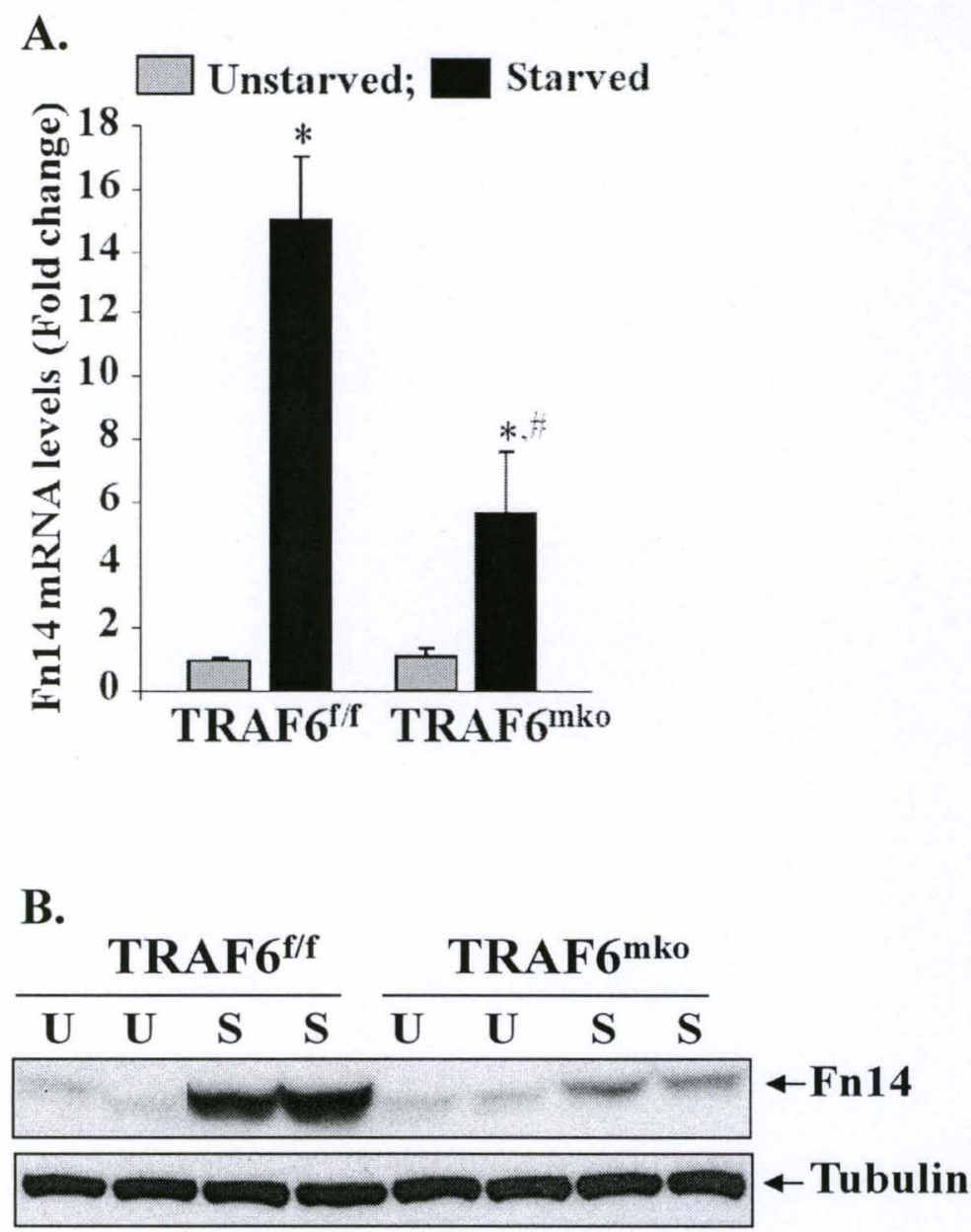

C.

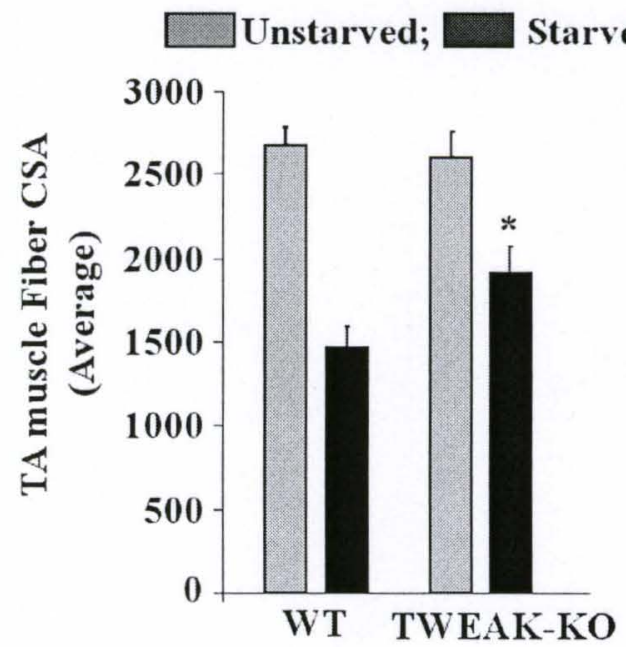

D.

$\square$ Unstarved; $\square$ Starved

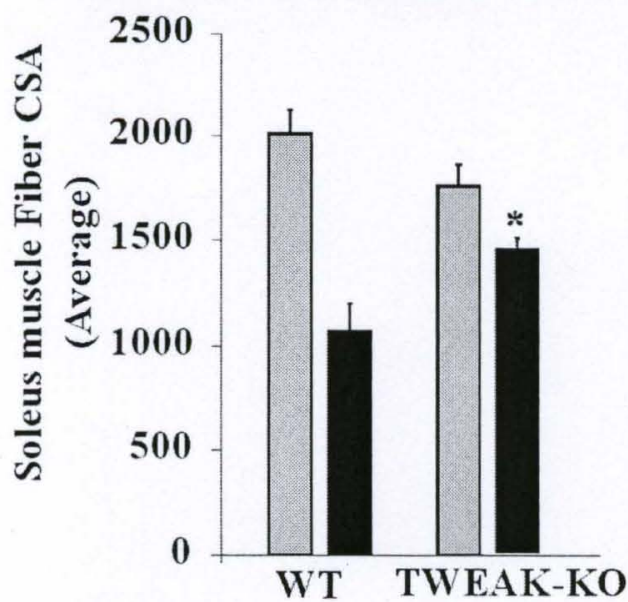



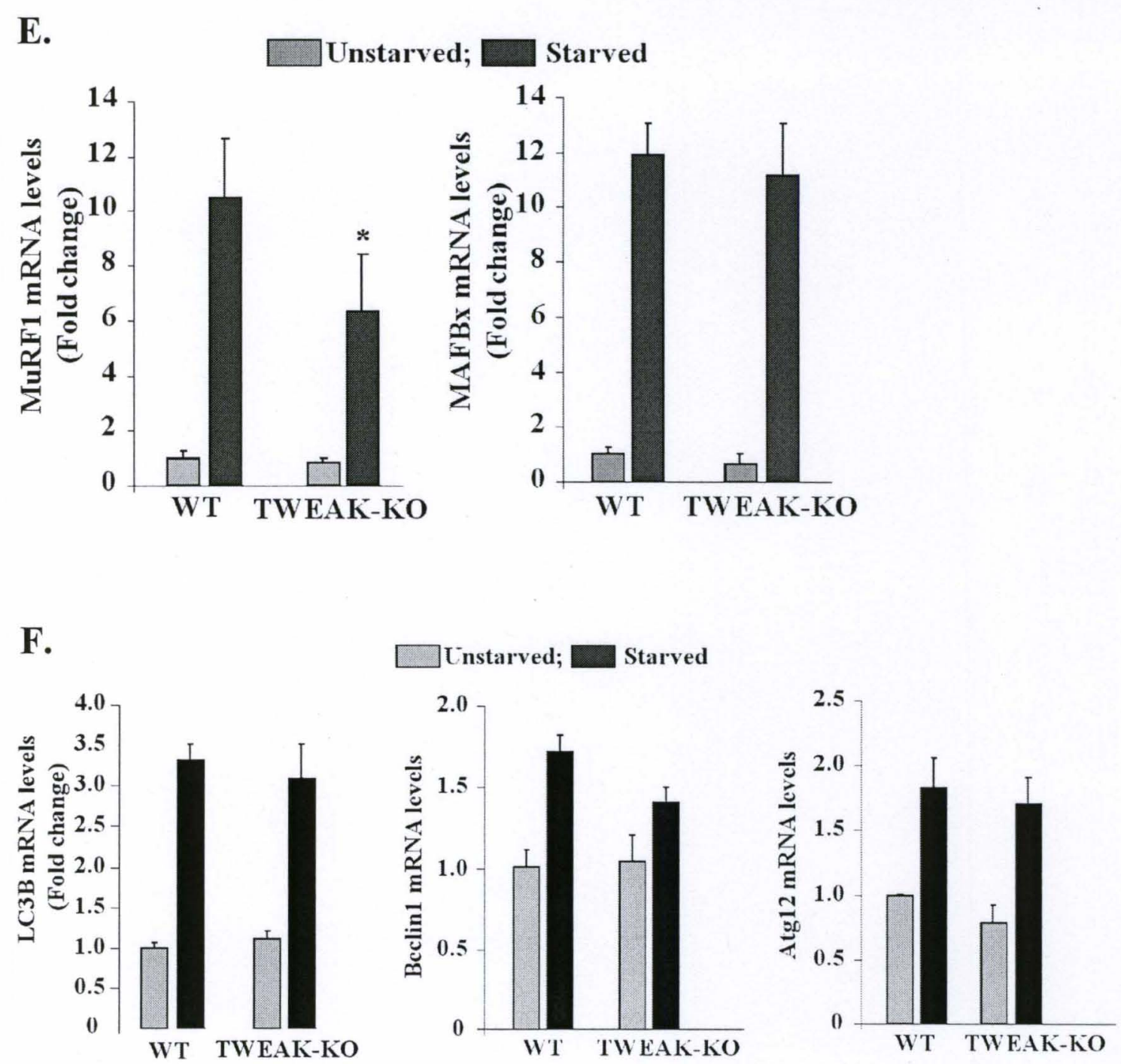

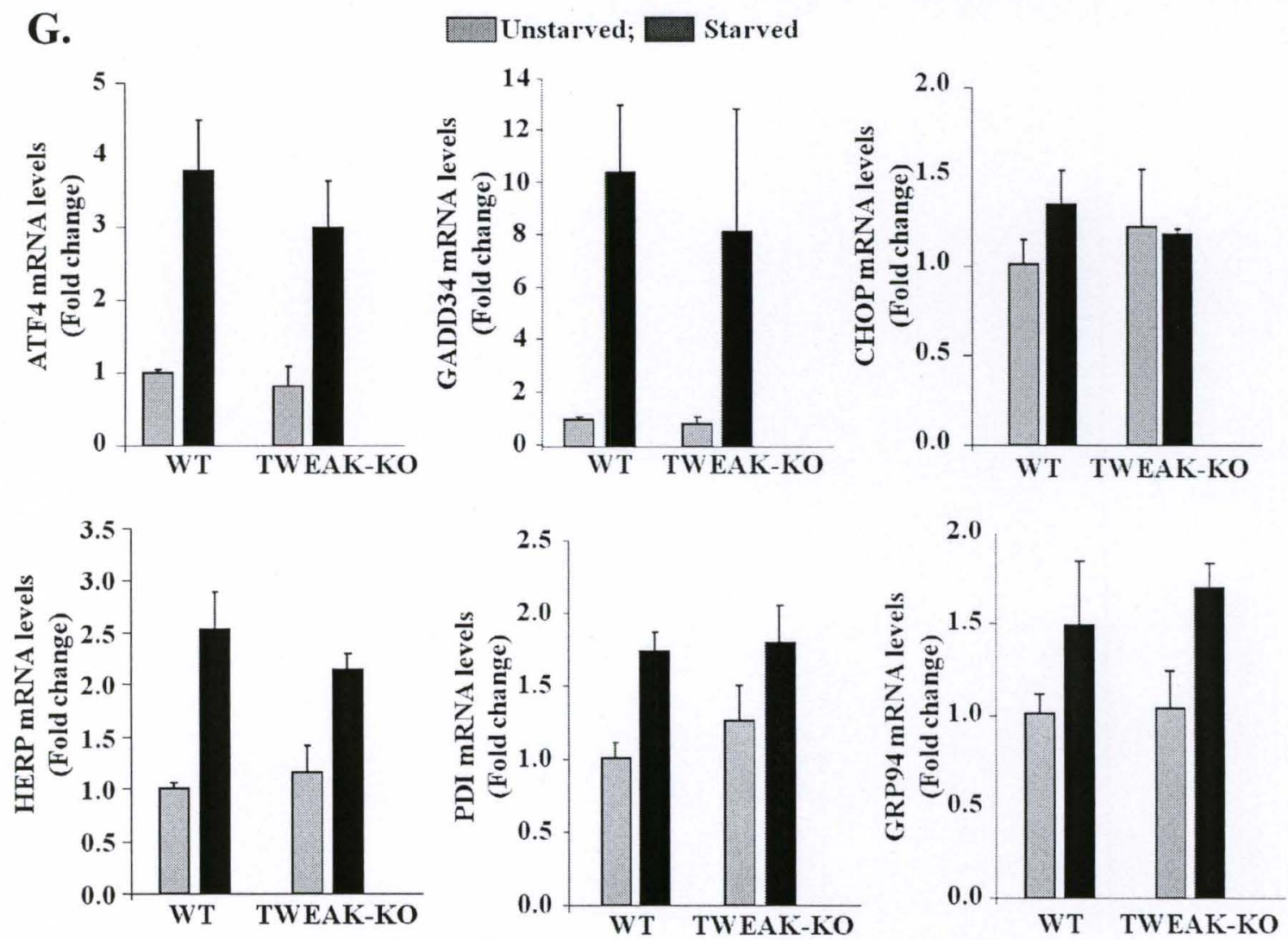
Figure 3.4: Activation of TWEAK-Fn14 system by TRAF6 in skeletal muscle of mice. TRAF6 $6^{\mathrm{t} / \mathrm{f}}$ and TRAF6 ${ }^{\mathrm{mko}}$ mice were fasted for $24 \mathrm{~h}$ and GA muscles isolated were analyzed for expression of Fn14. (A) Transcript levels of Fn14 were significantly reduced in starved GA muscle of TRAF6 ${ }^{\text {mko }}$ mice compared with TRAF6 ${ }^{\mathrm{f} / \mathrm{f}}$ mice. (B) Representative immunoblot presented here demonstrates reduced protein levels of Fn14 but not an unrelated protein tubulin in starved TA muscle of TRAF ${ }^{\text {mko }}$ mice

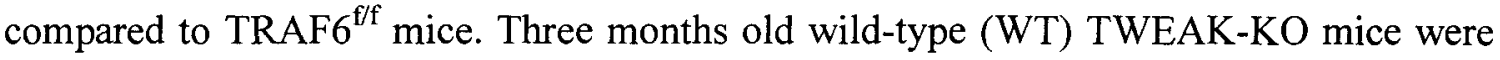
starved for $24 \mathrm{~h}$. Quantification of mean fiber CSA in H\&E-stained sections of (C) TA muscle, and (D) soleus muscle of WT and TWEAK-KO mice (N = 8 in each group). (E) Transcript levels of MuRF1 but not MAFBx was found to be significantly reduced fasted muscle of TWEAK-KO mice compared to WT mice. Error bars represent SD. ${ }^{\#}<0.01$, values significantly different from GA muscle of unstarved TRAF6 ${ }^{\mathrm{f} / \mathrm{f}}$ mice. ${ }^{*} \mathrm{p}<0.05$, values significantly different from GA muscle of starved TRAF $6^{\mathrm{f} / \mathrm{f}}$ mice. Expression of autophagy-related genes is starved muscle is independent of TWEAK-Fn14 system. (F) Three months old WT and TWEAK-KO mice were starved for 24 hrs and TA muscle was isolated for biochemical analyses. Starvation-induced increase in transcript levels of LC3B, Beclin1 and Atg12 did not show any significant difference between WT and TWEAK-KO mice. Ablation of TWEAK does not influence ER-stress genes in starved skeletal muscle of mice. (G) QRT-PCR analyses demonstrate that there was no significant difference in transcript levels of ATF4, GADD34, CHOP, HERPS, PDI and GRP94 in TA muscle of WT and TWEAK-KO mice in response to starvation. $\mathrm{N}=4$ in each group. Error bars represent SD. 
A.

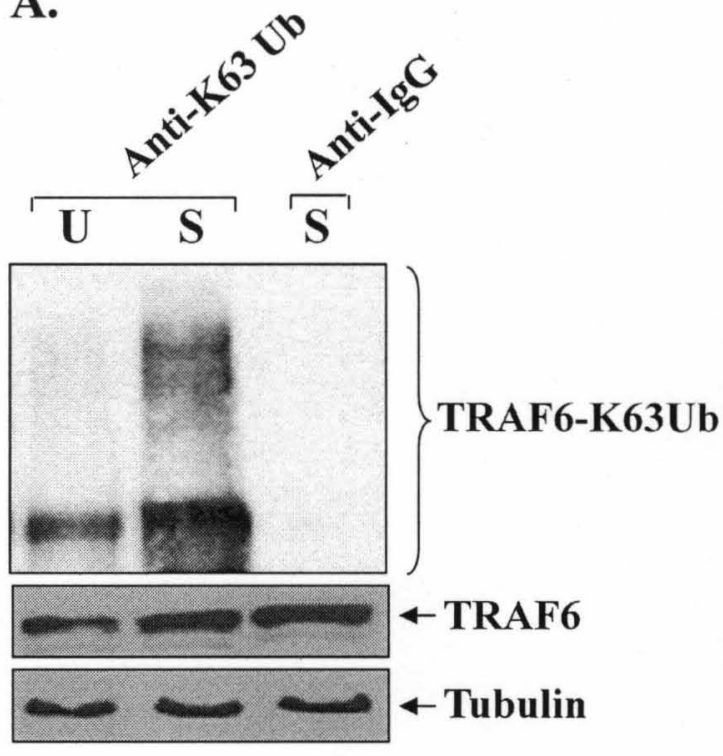

B.

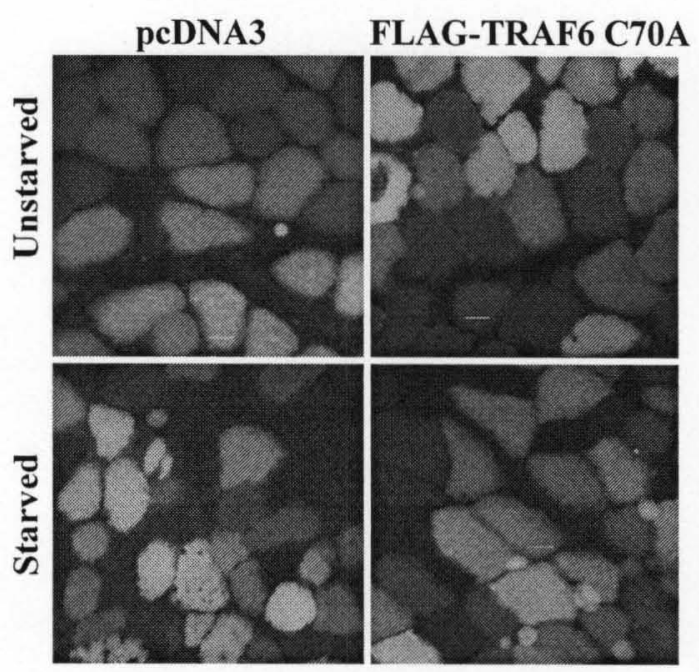

C.

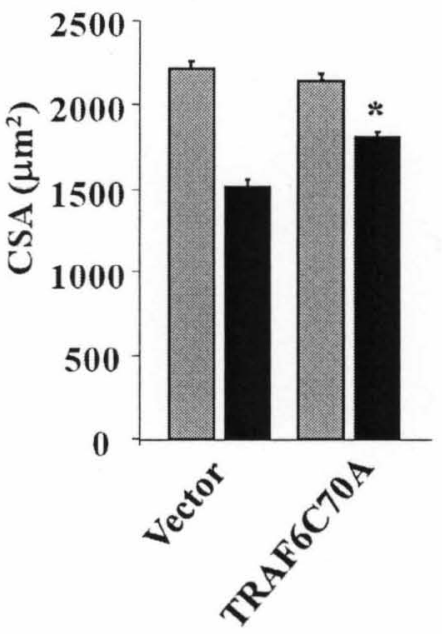




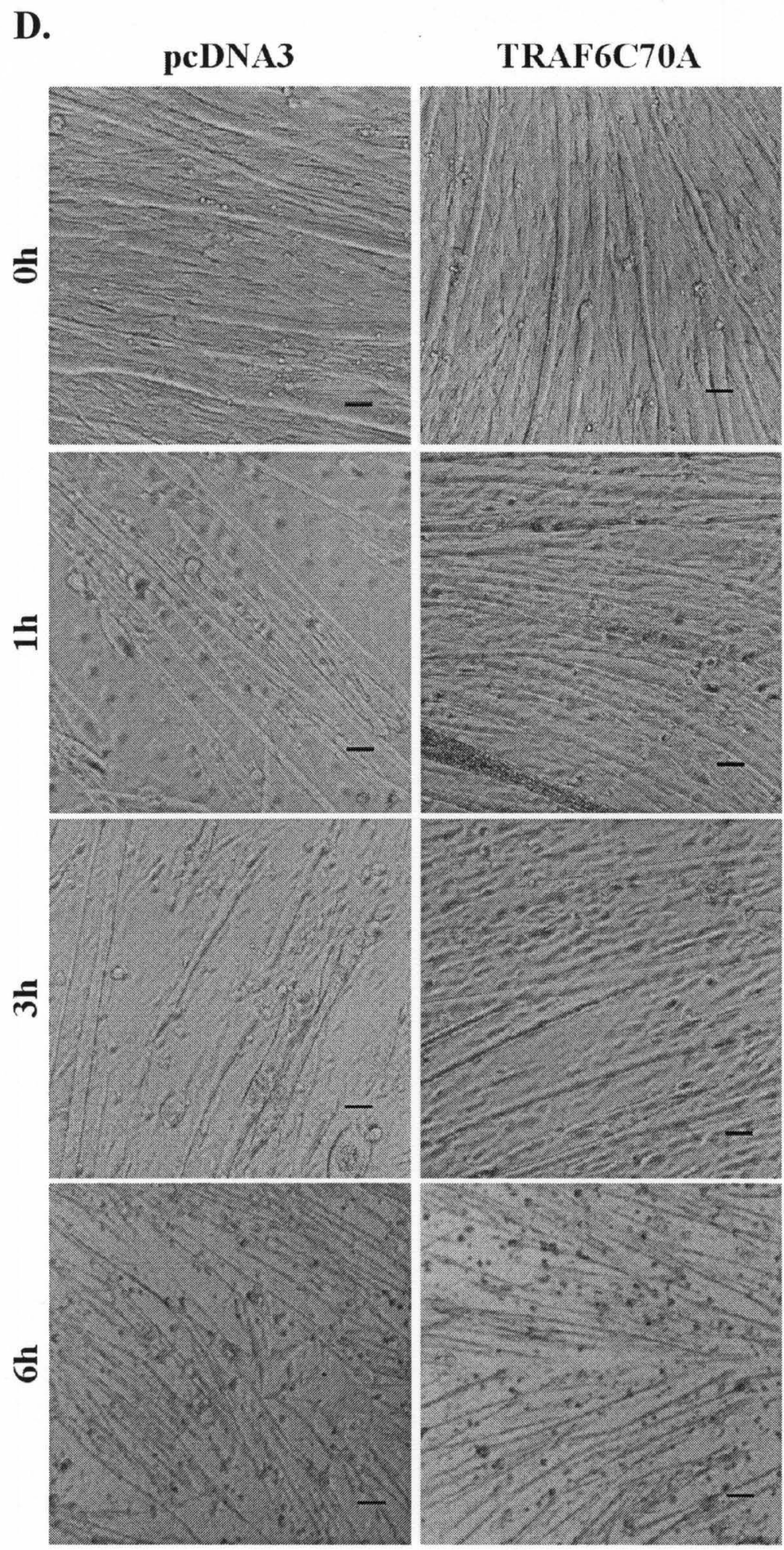



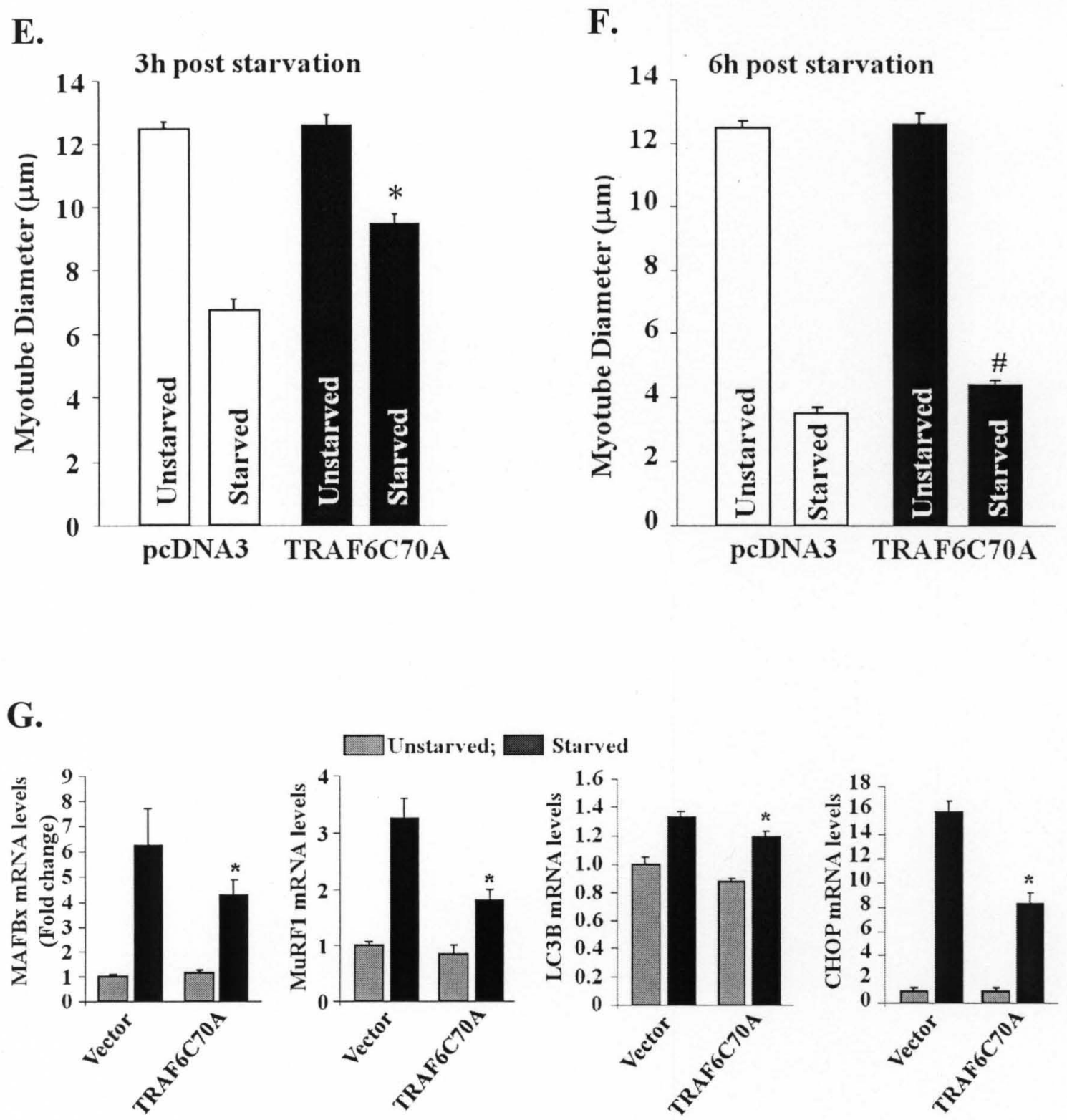

Figure 3.5: TRAF6 E3 ubiquitin ligase activity is required for starvation-induced muscle atrophy. Three months old mice were fasted for 24 hours. (A) TA muscle extracts prepared from unstarved and starved mice were immunoprecipitated with TRAF6 antibody followed by Western blotting using an antibody that recognizes only K63-linked ubiquitinated substrates (top panel). Western blotting using anti-TRAF6 in total muscle extracts (middle) and anti- $\alpha$-tubulin (bottom). U, unstarved; S, Starved. (B) TA muscles of C57BL6 mice were electroporated with plasmids expressing EGFP and FLAG-TRAF6C70A mutant. Ten days later, mice were fasted for $24 \mathrm{~h}$ and myofibers expressing EGFP in combination with pCDNA3 or FLAG-TRAF6C70A were analyzed by fluorescence microscopy. (C) Quantification of fiber cross-sectional area (CSA) of transfected TA muscle of TRAF $6^{\mathrm{f} / \mathrm{f}}$ and TRAF $6^{\mathrm{mko}}$ mice. (D) $\mathrm{C} 2 \mathrm{C} 12$ myotubes transfected with vector alone (i.e. pcDNA3) or TRAF6C70A plasmid were incubated in 
PBS. Representative phase contrasts pictures taken at different time point after addition of PBS are shown here. Myotube diameter in vector or TRAF6C70A-expressing myotubes was measured after (E) $3 \mathrm{~h}$ and (F) $6 \mathrm{~h}$ of addition of PBS. " $\mathrm{p}<0.01$, values significantly different from $3 \mathrm{~h}$ starved myotubes transfected with pcDNA3 alone. " $<<0.05$, values significantly different from $6 \mathrm{~h}$ starved myotubes transfected with pcDNA3 alone. (G) Cultured $\mathrm{C} 2 \mathrm{C} 12$ myoblasts were transfected with vector alone or TRAF6C70A mutant, differentiated into myotubes, and starved for $6 \mathrm{~h}$ in PBS. Starvation-induced increase in mRNA levels of MAFBx, MuRF1, LC3B and CHOP was significantly inhibited in TRAF6C70A-transfected cultures compared to those with vector alone. Error bars represent SD. ${ }^{*} p<0.05$, values significantly different from starved myotubes transfected with vector alone. Scale bar: $20 \mu \mathrm{m}$. 


\section{CHAPTER FOUR}

\section{SPECIFIC INHIBITION OF TRAF6 IMPROVES SKELETAL MUSCLE REGENERATION THROUGH PROMOTING M2c MACROPHAGE PHENOTYPE}

\subsection{INTRODUCTION}

Skeletal muscle in vertebrates is a terminally differentiated but highly regenerative tissue (146). Postnatal growth or regeneration of myofibers following injury is facilitated by a population of undifferentiated muscle precursor cells, commonly referred to as satellite cells. Satellite cells reside between the plasma membrane and basal lamina in a relatively quiescent, non-proliferative state (146-148). In response to injury, satellite cells are activated from quiescence, re-enter the cell cycle, and proliferate to generate a pool of myoblasts, which then fuse with each other or with damaged myofibers resulting in muscle repair and growth (146). Satellite cell activities are dynamically regulated by signals released from both the damaged muscle as well as other cell types either resident in the muscle or recruited to assist in clearing the damaged myofibers (149-151). However, the signaling mechanisms originating in damaged or regenerating myofibers and their role in regulation of specific regenerative responses is less explored.

Innate immune response which starts within hours of muscle injury is critical for efficient muscle regeneration (152). Among the immune cells infiltrated in injured 
myofibers, neutrophils are mostly phagocytic which assist in removal of cellular debris and matrix remodeling (152). In contrast, macrophages play more complex roles in muscle injury and regeneration. Although it remains unclear what confers the distinction between various effects of macrophages on injured muscles, diversity in macrophage phenotype provides an explanation for their differential roles. It has been suggested that initial inflammatory response in an injured muscle is driven by Th1 cytokines (153). Th1 cytokines such as tumor necrosis factor (TNF)- $\alpha$ and interferon- $\gamma$ (IFN- $\gamma$ ) stimulate the activation of phagocytic macrophage phenotype (M1) that invade the injured tissue first and stay at elevated concentration from $24 \mathrm{~h}$ to $48 \mathrm{~h}$ post-injury after which their level starts declining $(152,154)$. Although M1 macrophages contribute to muscle repair by removing cellular debris, they can also promote muscle injury by producing nitric oxide through induction of inducible nitric oxide synthase (155). In an injured muscle microenvironment, the shift from phagocytic to regenerative phase coincides with macrophage phenotype transition from M1 to M2. M2 macrophages have three functionally specialized subtypes (54); M2a macrophages, activated by Th2 cytokines IL4 and IL-13 can accelerate muscle repair. M2b macrophages which are activated by immune complexes and M2c macrophages, activated by IL-10 or transforming growth factor- $\beta$ (TGF- $\beta$ ), promote muscle regeneration by deactivating M1 phenotype $(54,152)$.

In the recent years, significant progress has been made to understanding the role of various signaling pathways such as nuclear factor-kappa B (NF-kB), p38 mitogenactivated protein kinase (MAPK), and phosphoinositide 3-kinase (PI3K)/Akt. While p38 MAPK and Akt are the positive regulators of myogenic differentiation $(49,156,157)$, the activation of classical NF- $\mathrm{KB}$ pathway inhibits the process of myogenesis $(64,158)$. 
However, except in the case of NF-kB where its targeted deletion in adult myofibers improved regeneration (86), majority of other studies were performed employing myoblast/satellite cell specific-knockout mice or whole-body knockout mouse models where no distinction was observed in the signaling originated in muscle progenitor cells or the injured myofibers $(157,159)$. Furthermore, little is known about the proximal and intermediate molecular events involved in the activation of various pathways in injured/regenerating myofibers.

Tumor necrosis factor receptor-associated factors (TRAFs) are a family of seven (i.e. TRAF1-7) conserved adaptor proteins which act as signaling intermediates for TNFR superfamily members and many other receptor-mediated events leading to downstream activation of NF- $\mathrm{kB}, \mathrm{PI} 3 \mathrm{~K} / \mathrm{Akt}$, and MAPK (160). Distinct from other TRAFs, TRAF6 is unique since it mediates toll-like receptor (TLR)/interleukin-1 receptor (IL-1R) superfamily signaling (6). TRAF6 was also observed to be highly regulated during myogenesis in a recent study in our lab. Proliferating myoblasts express high levels of TRAF6 which are dramatically reduced upon their differentiation into myotubes. On the other hand, the expression of TRAF6 is dramatically increased in specific catabolic conditions leading to loss of muscle mass. This paradox leads to an assumption that while TRAF6 may be required for myoblast proliferation and differentiation, its function is redundant in matured myotubes and could be catabolic in stress conditions. This assumption gains more validity especially due to involvement of TRAF6 in various cellular mechanisms in a regeneration program $(54,80,154,155)$. An injury-induced regeneration program involves innate immune response as well as interplay of several signaling cascades. However, the molecular mechanisms regulated by 
TRAF6 in phagocytic and regenerative phase of an injured muscle is completely unknown.

In this study, we have investigated the role and the mechanisms by which TRAF6 regulates regeneration of adult skeletal muscle. To distinguish the role of TRAF6mediated signaling in adult myofibers and satellite cells, we have used mice in which TRAF6 was depleted either in differentiated myofibers or in satellite cells early during embryonic development. Our results indicate that depletion of TRAF6 dramatically improves skeletal muscle regeneration in response to injury. Furthermore, our results also indicate that specific inhibition of TRAF6 leads to increased proportion of satellite cells and $\mathrm{M} 2 \mathrm{c}$ macrophages in regenerative phase. 


\subsection{MATERIALS AND METHODS}

\section{Mice}

Generation of transgenic Floxed TRAF6 $\left(\mathrm{TRAF}^{\mathrm{f} / \mathrm{f}}\right.$ ) and muscle specific knockout for TRAF6 (TRAF6 ${ }^{\text {mko }}$ ) mice have been described previously (117). Developmental muscle knock-out of TRAF6 (TRAF6 ${ }^{\mathrm{dmko}}$ ) mice were generated by breeding Myf5-Cre (strain B6.129S4-Myf $5^{t m 3(c r e) S o r} / \mathrm{J}$ ) with $\mathrm{TRAF} 6^{\mathrm{f} / \mathrm{f}}$. These mice were bred for several generations to obtain Myf5-Cre-TRAF6 ${ }^{\mathrm{f} / \mathrm{f}}$ mice which were designated as TRAF6 ${ }^{\mathrm{dmko}}$. All mice were in the C57BL/6 background and their genotype was determined by PCR from tail DNA. At the age of 8 weeks, $100 \mu \mathrm{l}$ of $10 \mu \mathrm{M}$ cardiotoxin (CalBiochem) dissolved in phosphate-buffered saline (PBS) was injected into the tibial anterior (TA) muscle to induce necrotic injury. At various time points, TA muscle was collected from euthanized mice for biochemical and histological studies. All experimental protocols with mice were approved in advance by the Institutional Animal Care and Use Committee at University of Louisville.

\section{Cell culture}

Primary myoblasts isolated from hind limb skeletal muscles of mice were grown in myoblast growth medium (Ham's F10 medium containing 10\% FBS supplemented with $5 \mathrm{ng} / \mathrm{ml}$ basic-FGF) for selection of pure population. Upon selection, these cells were cultured in 1:1 ratio in MGM + GM (DMEM+10\% FBS) till confluence. To induce differentiation, the cells were incubated in differentiation medium $(2 \%$ horse serum in DMEM) for $96 \mathrm{~h}$ as described (117). After $96 \mathrm{~h}$ of differentiation, myotubes were stained for myosin heavy chain (using MF20 antibody) and DAPI as described earlier (98, 161). 
Proliferating primary myoblasts were stained for MyoD and DAPI. Stained myoblasts and myotubes were examined by morphometric analyses.

\section{Cell Proliferation Assay}

Cell proliferation efficiency was determined using CyQUANT NF Cell Proliferation Assay Kit (Molecular Probes Inc., OR, USA) following the manufacturer's instructions. Briefly, primary myoblasts were seeded in a 96-well plate and grown for 96 hours. Proliferation of the myoblasts was determined by the CyQUANT NF cell proliferation assay by incubating adhered cells in dye binding solution (dye reagent in

HBSS buffer) at $37^{\circ} \mathrm{C}$ for $1 \mathrm{~h}$, then quantifying the fluorescence intensity (Ex $485 \mathrm{~nm} / \mathrm{Em}$ $530 \mathrm{~nm}$ ) on Spectramax M5 plate reader (Molecular Devices, CA, USA). The data were normalized relative to the proliferation of myoblasts from control animals.

\section{CK Assay}

The serum level of creatine kinase (CK) was determined using a commercially available kit (Stanbio Laboratory, TX, USA) as described (101). CK activity was expressed as $\mu-\mathrm{mol} / \mathrm{mg} / \mathrm{min}$.

\section{Electrophoretic Mobility Shift Assay (EMSA)}

NF- $\kappa \mathrm{B}$ activation was analyzed by EMSA. Detailed protocol for preparation of nuclear and cytoplasmic extracts from skeletal muscle tissues and cultured myotubes and performing EMSA has been described in detail in our previous publications (98, 100, 101). In brief, $20 \mu \mathrm{g}$ of nuclear extracts prepared from control or CTX-injected TA 
muscle were incubated with $16 \mathrm{fmol}$ of ${ }^{32} \mathrm{P}$ end-labeled NF- $\kappa \mathrm{B}$ consensus oligonucleotide (Promega) at $37^{\circ} \mathrm{C}$ for $30 \mathrm{~min}$, and the DNA-protein complex was resolved on a $7.5 \%$ native polyacrylamide gel. The radioactive bands from the dried gel were visualized and quantified by PhosphorImager (GE Health Care) using ImageQuant TL software.

\section{Fluorescence Activated Cell Sorting}

Activated satellite cells were analyzed by FACS as described (162). Satellite cells or macrophages were isolated from TA muscle of $\mathrm{TRAF}^{\mathrm{f} / \mathrm{f}}, \mathrm{TRAF}^{\mathrm{mko}}$ or TRAF $6^{\mathrm{dmko}}$ mice TA muscles following cell preparation protocol as previously described. Approximately $2 \times 10^{6}$ cells were incubated in DMEM (supplemented with $2 \%$ FBS and $25 \mathrm{mM}$ 4-(2-hydroxyethyl)-1-piperazineethanesulfonic acid)) and immunostained with antibodies against CD140a, CD45, CD31, Ter-119, CD11c and CD206 (FITC or PEconjugated, eBiosciences) and $\alpha 7 \beta 1$-integrin (MBL International) conjugated tandem-PE respectively. FACS analyses were performed on a C6 Accuri cytometer equipped with three lasers. Activated satellite cells were sorted (purity $>90 \%$ ) using antibody against $\alpha 7 \beta 1$-integrin for selection and cultured for further studies.

\section{Indirect Immunofluorescence and Histomorphometric Analyses}

All experiments involving the studies on $\mathrm{TRAF}^{\mathrm{f} / \mathrm{f}}, \mathrm{TRAF}^{\mathrm{mko}}$ and TRAF6 ${ }^{\text {dmko }}$ mice were conducted on at least six mice per group. Cardiotoxin (CTX) or PBS alone injected TA muscle were removed, frozen in isopentane cooled in liquid nitrogen and sectioned in a microtome cryostat. For the assessment of tissue morphology or visualization of fibrosis, $10-\mu \mathrm{m}$-thick transverse sections of muscles were stained 
respectively with the Hematoxylin and Eosin (H\&E) and examined under Nikon Eclipse TE 2000-U microscope (Nikon). Fiber cross-sectional area was measured in H\&E-stained TA muscle sections using Nikon NIS Elements BR 3.00 software (Nikon). For each muscle, mean fiber cross-sectional area (CSA) was calculated by analyzing 200 to 250 myofibers as described $(53,99)$. The extent of fibrosis in transverse cryosections of TA muscle determined using Mason's Trichrome staining kit following a protocol suggested by the manufacturer (Richard-Allan Scientific). For the detection of macrophages in TA cryosections, anti-F4/80 (dilution 1:100; clone CI:A3-1, AbD Serotec) was used in conjunction with the VECSTAIN ABC staining kit (Vector) with 3,3'-diaminobenzidine substrate according to the manufacturer's protocol. For immunohistochemical study, the sections were blocked in $1 \%$ bovine serum albumin in PBS for $1 \mathrm{~h}$, and incubated with anti-TRAF6 (1:200, Millipore), anti-CD68 (1:200, AbD Serotec), anti-E-MyHC (1:50 dilution, Developmental Studies Hybridoma Bank, University of Iowa, Iowa City, IA), anti-MF20 (1:250, DSHB, Iowa) and anti-MyoD (1:250, santa cruz) in blocking solution at $4^{\circ} \mathrm{C}$ overnight under humidified conditions. The sections were washed briefly with PBS before incubation with Alexa Fluor ${ }^{(} 488$ or 594-conjugated secondary antibody (1:3000, Invitrogen) for $1 \mathrm{~h}$ at room temperature and then washed 3 times for 30 minutes with PBS. The slides were mounted using fluorescence medium (Vector Laboratories) and visualized at room temperature on Nikon Eclipse TE 2000-U microscope (Nikon), images were captured on a digital camera (Nikon Digital Sight DS-Fil), and images were analyzed using Nikon NIS Elements BR 3.00 software (Nikon). Image levels were equally adjusted using Abode Photoshop CS2 software (Adobe). 


\section{Primary Myoblast Isolation}

Satellite cells were isolated from hind limb muscles of 8-week old mice using a method as described $(163,164)$. Briefly, mice were sacrificed and TA, gastrocnemius, and extensor digitorum longus muscles were isolated. Excess connective tissue and fat were cleaned in sterile PBS followed by mincing of skeletal muscle in Dulbecco's Modified Eagle's Medium (DMEM) and enzymatic dissociation with collagenase and $0.1 \%$ Pronase. The digested slurry was spun, pelleted and triturated several times and then passed through $70 \mu \mathrm{M}$ cell strainer (BD Falcon). The filtrate was spun at $1000 \times \mathrm{g}$ and resuspended in growth medium containing basic fibroblast growth factor $(5 \mathrm{ng} / \mathrm{ml})$. Cells were first re-fed after 3 days of initial plating. During first few passages cells were also enriched by pre-plating.

\section{RNA Isolation and Quantitative Real-time PCR (QRT-PCR)}

RNA isolation and QRT-PCR were performed using a method as previously described $(53,165)$.

\section{Western Blot}

Quantitative estimation of specific protein was done by Western blot using a method as previously described $(44,99,101)$. TA muscle were washed with PBS and homogenized in lysis buffer A [50 mM Tris- $\mathrm{Cl}(\mathrm{pH} 8.0), 200 \mathrm{mM} \mathrm{NaCl}, 50 \mathrm{mM} \mathrm{NaF}, 1$ $\mathrm{mM}$ dithiotheritol (DTT), $1 \mathrm{mM}$ sodium orthovanadate, $0.3 \%$ IGEPAL, and protease inhibitors]. Approximately, $100 \mu \mathrm{g}$ protein was resolved on each lane on 8-10\% SDSPAGE, electrotransferred onto nitrocellulose membrane and probed using anti-MF20 or 
anti-E-MyHC (1:100, Developmental Studies Hybridoma Bank), anti-TRAF6(1:1000, Millipore), anti-phospho-Akt (1:1000, Cell Signaling, Inc), anti-total Akt (1:1000, Cell Signaling, Inc), anti-phospho p38 (1:1000, Cell Signaling, Inc), anti-total p38 (1:1000, Cell Signaling, Inc.), anti-p65 (1:1000, Santa Cruz) and anti- $\alpha$-tubulin (1:2000, Cell Signaling, Inc.) and detected by chemiluminescence.

\section{Statistical Analyses}

Results are expressed as mean \pm standard deviation (SD). Statistical analyses used Student's $t$-test to compare quantitative data populations with normal distribution and equal variance. A value of $P<0.05$ was considered statistically significant unless otherwise specified. 


\subsection{RESULTS}

\subsubsection{Expression of TRAF6 is increased in regenerating skeletal muscle after injury}

An acute injury to skeletal muscle is followed by a well-orchestrated series of events which facilitates rapid repair and regeneration of injured muscle. We first investigated how the expression of TRAF6 is affected in skeletal muscle in response to injury. Six-week old wild type mice were given intramuscular injection of cardiotoxin (CTX) or saline alone in the tibial anterior (TA) muscle. After 5d of CTX injection, the TA muscle was isolated and used to study TRAF6 expression. QRT-PCR analysis using Myh4 gene as an internal control, we found that the mRNA levels of TRAF6 are significantly increased in CTX-injected myofibers compared to those injected with saline alone (Figure 4.1 A). Furthermore, Western blot analysis of muscle extracts showed that protein levels of TRAF6 were dramatically increased in CTX-injected TA muscle compared to contralateral TA muscle injected with saline alone (Figure 4.1 B). To confirm that the expression of TRAF6 was indeed induced in injured myofibers, we also studied TRAF6 expression by immunohistochemical analysis. A dramatic increase in TRAF6 expression was observed in injured muscle when compared to uninjured control (Figure $4.1 \mathrm{C}$ ). Through immunostaining for CD68, a cell surface marker for macrophages (49), we also sought to examine the presence of myeloid cells and whether they express TRAF6 in injured muscle. Appearance of $\mathrm{CD}^{+} 8^{+}$cells indicates augmented infiltration of macrophages in injured muscle environment (Figure 4.1 C). In CTXinjured muscle, we also observed co-localization of both TRAF6 and CD68 indicating that increased expression of TRAF6 accompanies macrophage infiltration. It also suggests a possible contribution of infiltrating macrophages in enhancing the levels of TRAF6. However, there is no evidence that activated macrophages express TRAF6 at 
higher levels. Therefore, it is more likely that increased TRAF6 expression is contributed mainly by cells of myogenic origin. Together, these data suggest that CTX-injury induces the expression of TRAF6 in skeletal muscles.

\subsubsection{Ablation of TRAF6 accelerates adult skeletal muscle regeneration in response to CTX-} mediated injury

CTX-mediated injury of muscle is followed by an immediate inflammatory response, satellite cell proliferation and myogenic differentiation within 3 days and new myotube formation within 5-7 days (166). A normal regeneration program is marked by newly formed myofibers with small diameter and central nuclei. To investigate the role of TRAF6-mediated signaling in injured muscle regeneration, we crossed floxed TRAF6 $\left(T_{R A F 6}{ }^{\mathrm{f} f \mathrm{f}}\right.$ ) with MCK-Cre mice to generate skeletal muscle specific TRAF6-knockout mice (henceforth TRAF $6^{\text {mko }}$ ) as described (117). The TA muscle of 8-week old $\mathrm{TRAF}^{\mathrm{mko}}$ and its littermate control mice $\left(\mathrm{TRAF}^{\mathrm{f} / \mathrm{f}}\right.$ ) were given intramuscular injection of saline alone or CTX in saline followed by isolation of muscle at different time points and processing them for Hematoxylin and Eosin (H\&E) staining. Intramuscular injection of CTX caused equal necrosis in TA muscle of both $\mathrm{TRAF}^{\mathrm{f} / \mathrm{f}}$ and $\mathrm{TRAF}^{\text {mko }}$ mice examined 2 days after CTX injection (data not shown). Interestingly, at 5 days post CTX injection, regeneration of TA muscle was found to be significantly improved in TRAF $^{\text {mko }}$ compared to $^{\text {TRAF }} 6^{\mathrm{f} / \mathrm{f}}$ (Figure $4.2 \mathrm{~A}$ ). In TRAF6 ${ }^{\mathrm{mko}}$, TA muscle regeneration was uniform with majority of newly formed myofibers having central nuclei while in $\mathrm{TRAF}^{\mathrm{f} / \mathrm{f}}$ regenerating central nucleated fibers were less in number with an increased density of interstitial nuclei which indicate a slower nuclear accretion of new myofibers 
and a higher concentration of infiltrated non-myogenic cells (Figure $4.2 \mathrm{~A}$ ). Improved regeneration in TA muscle of TRAF $6^{\mathrm{mko}}$ compared to $\mathrm{TRAF} 6^{\mathrm{f} / \mathrm{f}}$ mice was also evident at 10 and 21 days post CTX injection (Figure 4.2 A). Quantification of fiber cross-sectional area (CSA) of regenerated myofibers in H\&E-stained sections showed $\sim 36 \%$ improvement in TRAF6 ${ }^{\mathrm{mko}}$ mice compared to $\mathrm{TRAF}^{\mathrm{f} / \mathrm{f}}$ mice (Figure $4.2 \mathrm{~B}$ and $4.2 \mathrm{C}$ ). Moreover, the number of myofibers having more than one nucleus was significantly higher in regenerating TA muscle of $\mathrm{TRAF}^{\mathrm{mko}}$ mice compared with $\mathrm{TRAF}^{\mathrm{f} / \mathrm{f}}$ measured 5 days post CTX injection (Figure 4.2 D). Collectively, these results indicate that TRAF6 depletion in differentiated myofibers accelerates regeneration program in response to CTX injury.

\subsubsection{TRAF6 depletion leads to early restoration of muscle architecture}

An injured muscle goes through repair and regeneration after an acute injury. A shift from degenerative to regenerative stage is followed by transition of myogenic cells through expression of specific transcription factors and related genes. This pattern also mimics the embryonic development of muscles. It is shown that CTX-injury in mouse muscle stimulates expression of $\mathrm{MyoD}$ in satellite cells by $2^{\text {nd }}$ day of injury and afterwards a decline in MyoD expression and an increase in myogenin expression occurs by 3 days post-injury followed by a consequential and persistent elevation of embryonic form of myosin heavy chain (eMyHC) $(167,168)$. Furthermore, as a regenerating muscle progresses towards normal architecture, embryonic isoform of MyHC is replaced by adult isoform (14). To investigate whether depletion of TRAF6 causes any change in the temporal expression pattern of these markers, we examined TA muscle of TRAF $6^{\mathrm{f} / \mathrm{f}}$ and TRAF $^{\text {mko }}$ mice 5 days after CTX injection. Immunohistochemical staining revealed 
more uniform and abundant expression of eMyHC in regenerating muscle at 5 days after CTX injection in TA muscle of TRAF6 ${ }^{\text {mko }}$ compared to $\mathrm{TRAF}^{\mathrm{f} / \mathrm{f}}$ mice (Figure $4.3 \mathrm{~A}$ ). Western blot analyses showed that protein levels of eMyHC were considerably higher in TA muscle of $\mathrm{TRAF}^{\mathrm{mko}}$ compared to $\mathrm{TRAF}^{\mathrm{f} / \mathrm{f}}$ mice 5 days post CTX injection. Furthermore, we also determined the change in expression of adult isoform of $\mathrm{MyHC}$ and observed a noticeable improvement in its levels in TRAF6 ${ }^{\text {mko }}$ (Figure 4.3 B). QRT-PCR analyses also showed a significant increase in mRNA levels of eMyHC (i.e. Myh3) as well as myogenic regulatory factors $\mathrm{MyoD}$ and myogenin in CTX-injected TA muscle of $\mathrm{TRAF}^{\mathrm{mko}}$ mice compared to TRAF6 $6^{\mathrm{f} / \mathrm{f}}$ mice (Figure $4.3 \mathrm{C}$ ). Collectively, these results suggest that depletion of TRAF6 in adult myofibers accelerates the regenerative program in response to injury.

\subsubsection{Depletion of TRAF6 promotes satellite cell activation in adult skeletal muscle in response to injury}

Muscle injury is followed by the activation of satellite cells which is prerequisite for induction of efficient regeneration program in injured muscle (169). We next sought to determine whether signaling through TRAF6 plays a role in the activation of satellite cells in injured myofibers. A unique combination of cell surface markers (CD45, $\mathrm{CD} 31^{-}$, Ter1 $19^{-}, \alpha 7-\beta 1$ integrin $^{+}$) identify activated satellite cells in adult mouse skeletal muscle and allow their direct quantification by fluorescence-activated cell sorting (FACS) technique (170). We next quantified satellite cells in saline or CTX-injected TA muscle of $\mathrm{TRAF}^{\mathrm{f} / \mathrm{f}}$ and $\mathrm{TRAF}{ }^{\mathrm{mko}}$ mice. There was no significant difference in the number of satellite cells in uninjured TA muscle of $\mathrm{TRAF}^{\mathrm{f} / \mathrm{f}}$ and TRAF $6^{\mathrm{mko}}$ mice. Consistent with 
published reports, intramuscular injection of CTX significantly increased the number of satellite cells in TA muscle of both TRAF $6^{\mathrm{fff}}$ and TRAF $6^{\mathrm{mko}}$ mice. Interestingly, the number of $\alpha 7 \beta 1$ Integrin $^{+}$satellite cells was significantly higher in TRAF6 ${ }^{\text {mko }}$ mice compared to TRAF6 ${ }^{\mathrm{f} / \mathrm{f}}$ mice (Figure $4.4 \mathrm{~A}$ and $4.4 \mathrm{~B}$ ). Activated satellite cells increased from approximately $5 \%$ in uninjured muscles to roughly $8 \%$ in injured TA of TRAF $6^{\mathrm{t} / \mathrm{f}}$. This increase was further greater in TRAF6 ${ }^{\text {mko }}$, upto $13-14 \%$ of total cells (Figure $4.4 \mathrm{~B}$ ). The increased percentage of $\alpha 7 \beta 1$ Integrin $^{+}$cell population in TRAF $6^{\text {mko }}$ suggests an important role of TRAF6-mediated signaling in regulating satellite cell activation.

We next sought to determine whether depletion of TRAF6 exerts same effect on quiescent satellite cell population in injured muscle. Pax7 is a marker of quiescent satellite cells and depletes upon activation of these cells. By performing QRT-PCR, we measured transcript levels of Pax7 in TA muscle of TRAF6 $6^{\mathrm{fff}}$ and TRAF6 ${ }^{\mathrm{mko}}$ mice. There was no significant difference in the mRNA levels of Pax7 in uninjured muscle of $\mathrm{TRAF}^{\mathrm{fff}}$ and TRAF6 ${ }^{\mathrm{mko}}$ mice. However, the transcript levels of Pax7 were significantly increased in CTX-injected TA muscle of TRAF6 ${ }^{\mathrm{mko}}$ ( $\sim .5$ fold $)$ compared to TRAF6 $6^{\mathrm{flf}}$ ( 2 fold) (Figure $4.4 \mathrm{C}$ ). Comparable basal level of expression of Pax7 in uninjured TA muscle of mice suggests that TRAF6 depletion does not influence primary myogenesis (Figure $4.4 \mathrm{C}$ ). In contrast, the higher expression of Pax7 in injured TA muscle of TRAF $6^{\text {mko }}$ can be attributed to increased proliferation of activated satellite cells from which a subpopulation reenters into quiescence. Collectively, these results suggest that in injury-induced regeneration program where secondary myogenesis is critical for muscle repair and regeneration, TRAF6 negatively regulates satellite cell activation. 


\subsubsection{TRAF6-mediated signaling negatively regulates the activation of anti- inflammatory M2c macrophage in injured myofibers}

Infiltration of macrophages upon myofiber injury is required not only for the removal of damaged tissue but also for normal progression of regenerative phase. Macrophage phenotype transition also marks the shift from phagocytic to regenerative stage of muscle repair. While phagocytic M1 macrophages are involved in removal of cellular debris, they have no role after the onset of regenerative stage (49). In fact, persistent presence of M1 macrophages can aggravate muscle damage (171). M2 macrophages have functionally distinct subtypes. M2a macrophages are involved with tissue repair and fibrosis while anti-inflammatory M2c macrophages deactivate M1 population and promote myogenic proliferation (172).

To determine whether improved regeneration in TRAF6-depleted muscle is a consequence of a bias in macrophage activation, we quantified M1 and M2c macrophage populations in CTX-injured TA muscle. Three days post CTX-injection, a time point at which transition in macrophage phenotype occurs, we examined percentages of M1 and M2c macrophages by analyzing an $\mathrm{F} 4 / 80^{+}$population of macrophages for phenotype composition by FACS method. As shown in Figure 4.5 A and 4.5 B, M1 macrophages $\left(\mathrm{CD} 11 \mathrm{c}^{+}\right)$were significantly higher in CTX-injected TA muscle of TRAF6 $6^{\mathrm{t} / \mathrm{f}}(\sim 10 \%)$ compared to $\mathrm{TRAF}^{\mathrm{mko}}(\sim 7.7 \%)$. In contrast, concentration of $\mathrm{CD}^{\mathrm{m}} 26^{+} \mathrm{M} 2 \mathrm{c}$ macrophages was significantly elevated in TA muscle of TRAF6 ${ }^{\text {mko }}(\sim 4.6 \%)$ compared to $\mathrm{TRAF}^{\mathrm{f} / \mathrm{f}}(\sim 2 \%)$ mice measured 3 days after CTX injection. There was no significant difference in the composition of total activated macrophages in peritoneal cavity or uninjured TA muscle of $\mathrm{TRAF} 6^{\mathrm{f} / \mathrm{f}}$ and $\mathrm{TRAF}^{\mathrm{mko}}$ mice (data not shown). Since M2 
macrophages are known to have anti-inflammatory and pro-myogenic effects on skeletal muscle, their increased activation could be a potential reason for enhanced muscle regeneration in $\mathrm{TRAF}^{\mathrm{mko}}$ mice upon injury.

It has been consistently observed that inflammatory cytokines such as TNF- $\alpha$ and IL-1 $\beta$ promote M1 phenotype whereas IL-4 and IL-10 are known to induce M2c phenotype of macrophages. We investigated whether signaling through TRAF6 affects the expression of these cytokines in regenerating myofibers. Interestingly, we found that the expression levels of TNF- $\alpha$ and IL-1 $\beta$ and chemokines CCL2 and CCL5 which recruit M1 macrophages were significantly reduced in CTX-injected TA muscle of TRAF6 $^{\text {mko }}$ mice compared to TRAF6 ${ }^{\mathrm{f} / \mathrm{f}}$ mice (Figure $4.5 \mathrm{C}$ ). In contrast, there was no significant difference in the mRNA levels of IL-4 and IL-10 in TA muscle of TRAF6 ${ }^{\text {mko }}$ and TRAF $6{ }^{\mathrm{f} / \mathrm{f}}$ mice (Figure $4.5 \mathrm{C}$ ). These data suggest that ablation of TRAF6 inhibits the expression of cytokines (i.e. TNF- $\alpha$ and IL-1 $\beta$ ) involved in manifestation of M1 phenotype. Their reduced levels may favor transition of M1 to M2c phenotype leading to increased regeneration of skeletal muscle in $\mathrm{TRAF}^{\mathrm{mko}}$ mice.

\subsubsection{TRAF6 is required for the activation of NF- $\mathrm{KB}$ in injured skeletal muscle}

$\mathrm{NF}-\mathrm{kB}$ is a nuclear transcription factor involved in inducible expression of a plethora of genes including TNF- $\alpha$ and IL-1 $\beta$. Although the exact mechanisms remain unknown, the activation of NF-kB has been found to inhibit skeletal muscle regeneration in response to CTX-mediated injury. Since TRAF6 is a central regulator in the activation of a number of cell signaling pathways including NF- $\mathrm{kB}$, we next studied that how ablation of TRAF6 affects the activation of NF-kB. In addition, we also evaluated the 
activation of Akt and p38MAPK, which are the positive regulators of skeletal muscle regeneration. The DNA binding activity of NF- $\mathrm{KB}$ was studied by performing electrophoretic mobility shift assay (EMSA) whereas Akt and p38 MAPK activity was studied by performing Western blots using antibodies that recognize phosphorylated forms of p38MAPK or Akt kinase. Consistent with published reports, DNA-binding activity of NF- $\mathrm{KB}$ was significantly increased in CTX-injected TA muscle compared to those injected with saline alone. Interestingly, the activation of NF-kB was significantly reduced in TA muscle of TRAF $6^{\mathrm{mko}}$ mice compared to TRAF $6^{\mathrm{f} / \mathrm{f}}$ mice injected with $\mathrm{CTX}$ (Figure $4.6 \mathrm{~A}$ ). While the phosphorylation of both p38MAPK and Akt kinase was increased in CTX-injected myofibers, we found no significant difference in the level of phosphorylation of Akt or p38 MAPK in regenerating TA muscle of $\mathrm{TRAF}^{\mathrm{f} / \mathrm{f}}$ and $\mathrm{TRAF}^{\mathrm{mko}}$ mice (Figure $4.6 \mathrm{~B}$ ). Phosphorylation of $\mathrm{p} 65$ subunit of NF- $\mathrm{KB}$ was also increased in response to CTX-injury in TA muscle, but this increase was more pronounced in $\mathrm{TRAF}^{\mathrm{f} / \mathrm{f}}$ mice when compared to $\mathrm{TRAF} 6^{\mathrm{mko}}$ mice (Figure 4.6 B). Taken together, these results suggest that TRAF6 specifically affects the activation of NF- $\mathrm{kB}$ in injured/regenerating skeletal muscle of mice.

\subsubsection{Depletion of TRAF6 early during embryonic development delays muscle growth.}

TRAF6 is an important signaling molecule involved in the proliferation and differentiation of various cell types. A recent report has suggested that siRNA-mediated knockdown of TRAF6 reduces the proliferation and differentiation of $\mathrm{C} 2 \mathrm{C} 12$ myoblasts (173). To further evaluate the role of TRAF6 in skeletal muscle formation, we next studied the consequence of deletion of TRAF6 in muscle progenitor cells. Published 
reports suggest that Myf5-Cre allele faithfully recapitulates the expression pattern of endogenous Myf5 and is uniformly expressed in all proliferating myoblasts (162, 174, 175). We crossed TRAF6 ${ }^{\mathrm{f} / \mathrm{f}}$ mice with Myf5-Cre mice to generate TRAF6 developmental muscle knockout (henceforth TRAF6 ${ }^{\mathrm{dmko}}$ ) and littermate control (i.e. TRAF6 ${ }^{\mathrm{f} / \mathrm{f}}$ ) mice. We first investigated whether there is any developmental phenotype in skeletal muscle of $\mathrm{TRAF}{ }^{\mathrm{dmko}}$ mice. While no gross abnormality was observed, average fiber CSA in soleus and TA muscle was found to be significantly reduced in TRAF6 ${ }^{\text {dmko }}$ mice compared to littermate $\mathrm{TRAF}^{\mathrm{f} / \mathrm{f}}$ mice (Figure 4.7 A, 4.7 B). Surprisingly, there was no significant difference in average CSA of soleus and TA muscles of 10-week old TRAF6 ${ }^{\mathrm{f} / \mathrm{f}}$ and TRAF $^{\mathrm{dko}}$ mice (Figure $4.7 \mathrm{~A}, 4.7 \mathrm{C}$ ). These data suggest that deletion of TRAF6 in myoblasts may transiently attenuate skeletal muscle development potentially by affecting the proliferation and/or differentiation of myoblasts.

To address the role of TRAF6 in myogenesis, we prepared primary myoblasts from TRAF $6^{\mathrm{fff}}$ and TRAF6 ${ }^{\mathrm{dmko}}$ mice and studied their proliferation and differentiation. Primary myoblasts from $\mathrm{TRAF}^{\text {dmko }}$ mice showed significantly reduced proliferation compared to $\mathrm{TRAF}^{\mathrm{f} / \mathrm{f}}$ mice. Immunohistochemical staining revealed smaller number of proliferating myoblasts (identified as cells coimmunostained with antibodies for MyoD and DAPI) in primary myoblast culture from TRAF6 ${ }^{\text {dmko }}$ when compared to TRAF6 $6^{\text {t/f }}$ mice (Figure 4.7 D, E). We also analyzed proliferation efficiency of primary myoblasts and observed a significant reduction in myoblast proliferation efficiency of TRAF6 $6^{\text {dmko }}$ mice compared to $\mathrm{TRAF}^{\mathrm{t} / \mathrm{f}}$ mice (Figure $4.7 \mathrm{~F}$ ). Primary myoblasts were cultured at equal seeding density and were induced to differentiate into myotubes by incubation in differentiation medium (DM) for $96 \mathrm{~h}$. Myotube formation was significantly reduced in 
primary cultures from TRAF $6^{\mathrm{dmko}}$ mice compared to $\mathrm{TRAF} 6^{\mathrm{fff}}$ assayed by both by morphometric (i.e. myotube diameter) and biochemical (creatine kinase activity) methods (Figure 4.7 G-I). Collectively, these data indicate that TRAF6 is required for proliferation and differentiation of cultured myotubes.

4.3.8 TRAF6 signaling in differentiated myofibers plays a dominant role over myoblasts during adult skeletal muscle regeneration

We next investigated whether muscle regeneration in response to CTX-mediated injury was affected in TRAF6 ${ }^{\text {dmko }}$ mice. Adult (8-week old) TRAF6 ${ }^{\mathrm{f} / \mathrm{f}}$ and TRAF6 ${ }^{\mathrm{dmko}}$ mice were given intramuscular injection of CTX in TA muscle. After $5 \mathrm{~d}$ or $10 \mathrm{~d}$ of CTX injection, the TA muscle was isolated and subjected to morphometric analyses. Intriguingly, we found that skeletal muscle regeneration was dramatically improved in TA muscle of TRAF6 ${ }^{\mathrm{dmko}}$ mice compared to TRAF6 ${ }^{\mathrm{t} / \mathrm{f}}$ mice (Figure 4.8 A). Similar to $\mathrm{TRAF}^{\mathrm{mko}}$ mice, CTX-injected TA muscle of TRAF $6^{\text {dmko }}$ showed a significant increase in fiber CSA and number of regenerating myofibers containing more than one nuclei (Figure 4.8 B-D). Percentage of satellite cells was significantly increased in CTXinjected TA muscle of TRAF6 ${ }^{\text {dmko }}(\sim 14)$ compared to TRAF $6^{\text {f/f }}(\sim 8)$ mice (Figure $4.8 \mathrm{E}$ and $4.8 \mathrm{~F}$ ). Furthermore, quantification of M1 and M2c macrophages revealed reduction in M1 macrophages with simultaneous increase in proportion of M2c macrophages in CTX-injected TA muscle of TRAF $6^{\mathrm{dmko}}$ mice compared to TRAF $6^{\mathrm{f} / \mathrm{f}}$ mice (Figure $4.8 \mathrm{G}$ and $4.8 \mathrm{H})$. 


\subsection{CONCLUSION AND DISCUSSION}

This study describes a novel role for TRAF6 in orchestrating a new balance between inflammatory and myogenic responses in toxin-induced injury of skeletal muscle. Using conditional knock-out mouse model for TRAF6, we have provided first evidence that in injured skeletal muscles, TRAF6 modulates inflammatory response and negatively regulates secondary myogenesis. TRAF6 is a common signaling adaptor molecule in IL-1R/TLR and TNFR superfamily receptors (39, 80, 103). Signaling cascades mediated by these pathways and their role in innate immune response are well documented and are also implicated in skeletal muscle development and metabolism $(176,177)$. An acute injury to muscle develops a microenvironment which is regulated by a highly complex interplay between immune and myogenic responses. In an injured muscle microenvironment, there is a significant interaction between myeloid and myogenic cells and these two populations communicate using common signaling cues. Although the common extracellular denominators and downstream effectors in skeletal muscle injury are well explored, the proximal intracellular regulators of these signaling events in muscles are unknown and their effect on skeletal muscle regeneration is yet to be identified.

Our data reveal an important role of TRAF6 in myeloid-myogenic interaction and in secondary myogenesis. In injured muscle, an initial invasion of myeloid cells leads to removal of necrotic debris by neutrophils and M1 macrophages. Initial activation of proinflammatory macrophages is by Th1 cytokine and is uninfluenced by muscle cells, but as the regeneration progresses, cytokines produced by muscle cells may also contribute in prolonged activation and presence of M1 macrophages. TNF $\alpha$ is a major activator of M1 macrophages. In our TRAF $6^{\text {mko }}$ mouse model, a repressed expression of TNF $\alpha$ at day 5 
after injury (when regeneration peaks) indicates a reduction in its release from muscle cells. This will eventually lead to reduced activation of M1 macrophages and also implicate that TRAF6 may be involved in production of inflammatory cytokines in muscles and therefore can also determine the duration and extent of inflammatory phase in injured muscles.

Quiescent satellite cells are known to be activated after injury by HGF (hepatocyte growth factor), IGF-1 (insulin-like growth factor-1) and FGF (fibroblast growth factor) $(155,157,178)$. Post-activation, their proliferation and maturation is regulated by several positive and negative mediators. Activated by pro-inflammatory cytokines, NF-кB is a known inhibitor of myogenesis and myogenic differentiation (179, 180). Induction of NF- $\mathrm{KB}$ is regulated by different signaling contexts and TRAF6 is reported to mediate this activation through different downstream complexes $(10,18)$. In response to injury, pro-inflammatory cytokines released by macrophages or muscle cells induce NF- $\mathrm{KB}$ expression. Consistent with previously published reports, our data indicate an increase in NF- $\mathrm{KB}$ levels and its DNA binding activity; interestingly, its DNA binding activity was blunted significantly in absence of TRAF6 in regenerating skeletal muscle. It is not completely understood whether TNF $\alpha$-mediated activation of NF- $\mathrm{B}$ is independent of TRAF6. Indeed, we observed a repressed activation but not a complete inhibition of NF-kB in CTX-injected TA muscle of TRAF $6^{\text {mko }}$ mice. TRAF6 ablation; on the other hand, causes a significant induction in expression of p38MAPK which is a positive regulator of myogenesis. While this data is in contrast to the previous reports of TRAF6 dependent p38MAPK activation (181), it highlights TRAF6-mediated differential activation of downstream pathways (48). This context-dependent role of TRAF6 supports 
our observation that in injury-induced secondary regeneration of skeletal muscle, TRAF6 acts as a negative regulator.

Role of TRAF6 in innate immune response is extensively studied, but there is a dearth of information on its mediation in activation of different phenotypes of macrophages. In skeletal muscle regeneration, the macrophage phenotype shift plays a pivotal role since pro-inflammatory (M1) and anti-inflammatory (M2c) macrophages exert antagonistic effects on secondary myogenesis (155). Deactivation of M1 phenotype and activation of M2c phenotype creates an anti-inflammatory and pro-myogenic environment for rapid regeneration. Consistently, our data show that TRAF6 depletion repressed activator cytokine ( $\mathrm{TNF} \alpha$ ) of $\mathrm{M} 1$ macrophages and reduced their population in regenerating muscles. For the first time in this study, we also report an increase in M2c population in TRAF6-ablated injured/regenerating muscle, but in absence of a published evidence, it remains a subject for further exploration that how TRAF6 is involved in regulatory mechanisms that activate M2c phenotype of macrophages.

A cumulative effect of changes in molecular and cellular events imparted by TRAF6 deletion are observed in terms of significantly improved regeneration in TRAF6 conditional knock-out mice. TRAF6 depletion induced repression of pro-inflammatory factors, increased up-regulation of markers of myogenic differentiation and rapid regeneration marked by expedited restoration of muscle architecture. Ablation of TRAF6 also led to reduced deposition of transient ECM and thus rescued injury-induced transient fibrosis. Moreover, our results led to a very interesting observation that in developmental muscle knock-out for TRAF6 (TRAF $6^{\text {dmko }}$ ) mice, while absence of TRAF6 attenuates the proliferation and muscle formation efficiency, it also improves regeneration and repair by 
a significant margin. This indicates that TRAF6 may be involved in indirect orchestration of microenvironment in regenerating muscles. TRAF6 possibly regulates some muscleintrinsic factors which in turn influence inflammatory response in injured and regenerating muscles. Further studies will indeed be able to unearth the more subtle regulatory interactions of TRAF6 with components of myogenic and myeloid pools in regenerating skeletal muscle. Collectively, these indicate TRAF6 deletion induced a more efficient regeneration program.

In summary, TRAF6 mediated regulation of secondary myogenesis and regeneration unveiled by this study provides an unanticipated link between inflammatory and myogenic responses in an injured tissue microenvironment. Considering the importance and limited availability of therapeutic interventions that can influence the balance between inflammation and myogenesis in pathological conditions such as DMD (Duechene Muscular Dystrophy) (155), we believe that interventions targeting TRAF6mediated signaling will enhance the ability to improve pathological conditions in inflammatory muscle disorders. 
A.

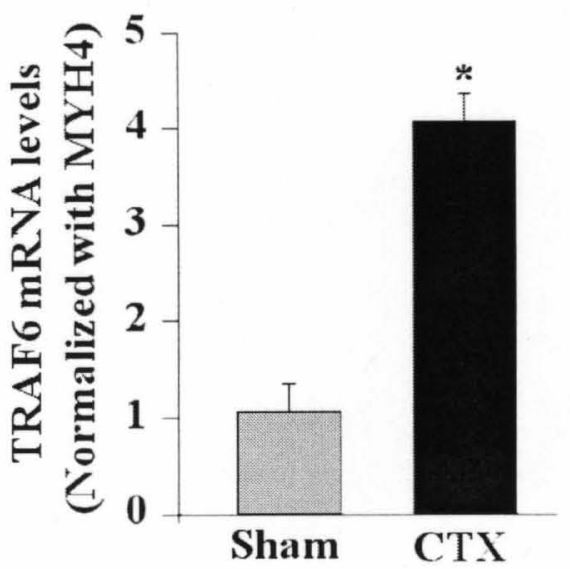

B.

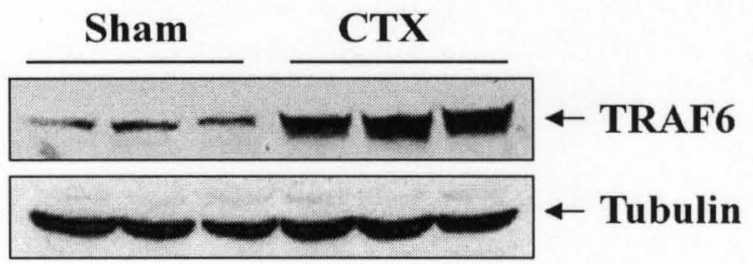

C.

Saline

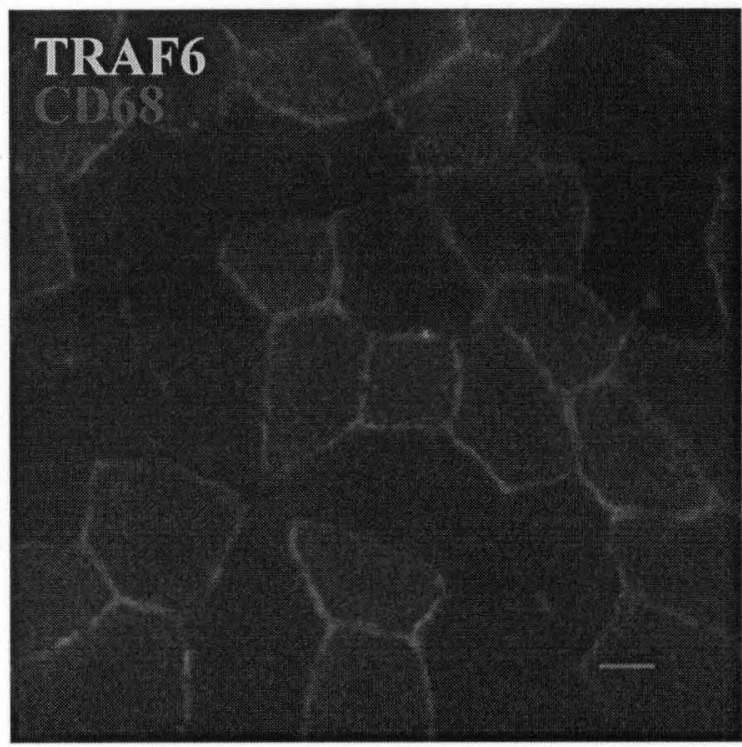

\section{CTX}

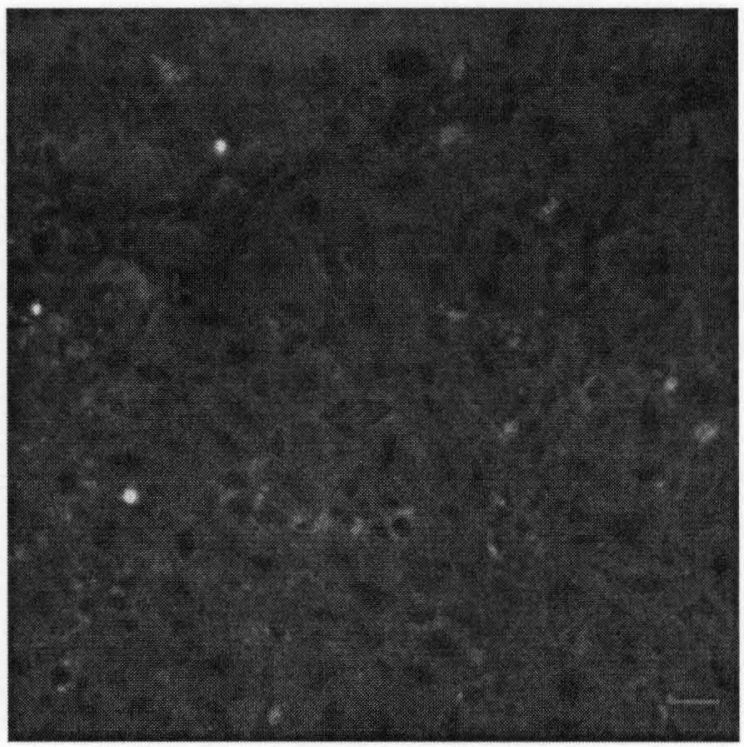

Figure 4.1: Necrotic muscle injury induces TRAF6 expression. (A) Transcript levels of TRAF6 in Tibialis anterior (TA) muscle of control and Ctx injected wild type mice. Error bars represent SD. $\mathrm{N}=3$ in each group. ${ }^{*} \mathrm{p}<0.01$, values significantly different from sham-injured muscle. (B) Western blot analyses of TA muscle extracts for TRAF6 protein at day 5 after Ctx injury. (C) Representative photomicrographs of immunostained sections of control and $\mathrm{Ctx}$-injured TA muscles at day 5 after injury $(\mathrm{N}=8)$. Sections immunostained with anti-TRAF6 and anti-CD68. 
A.

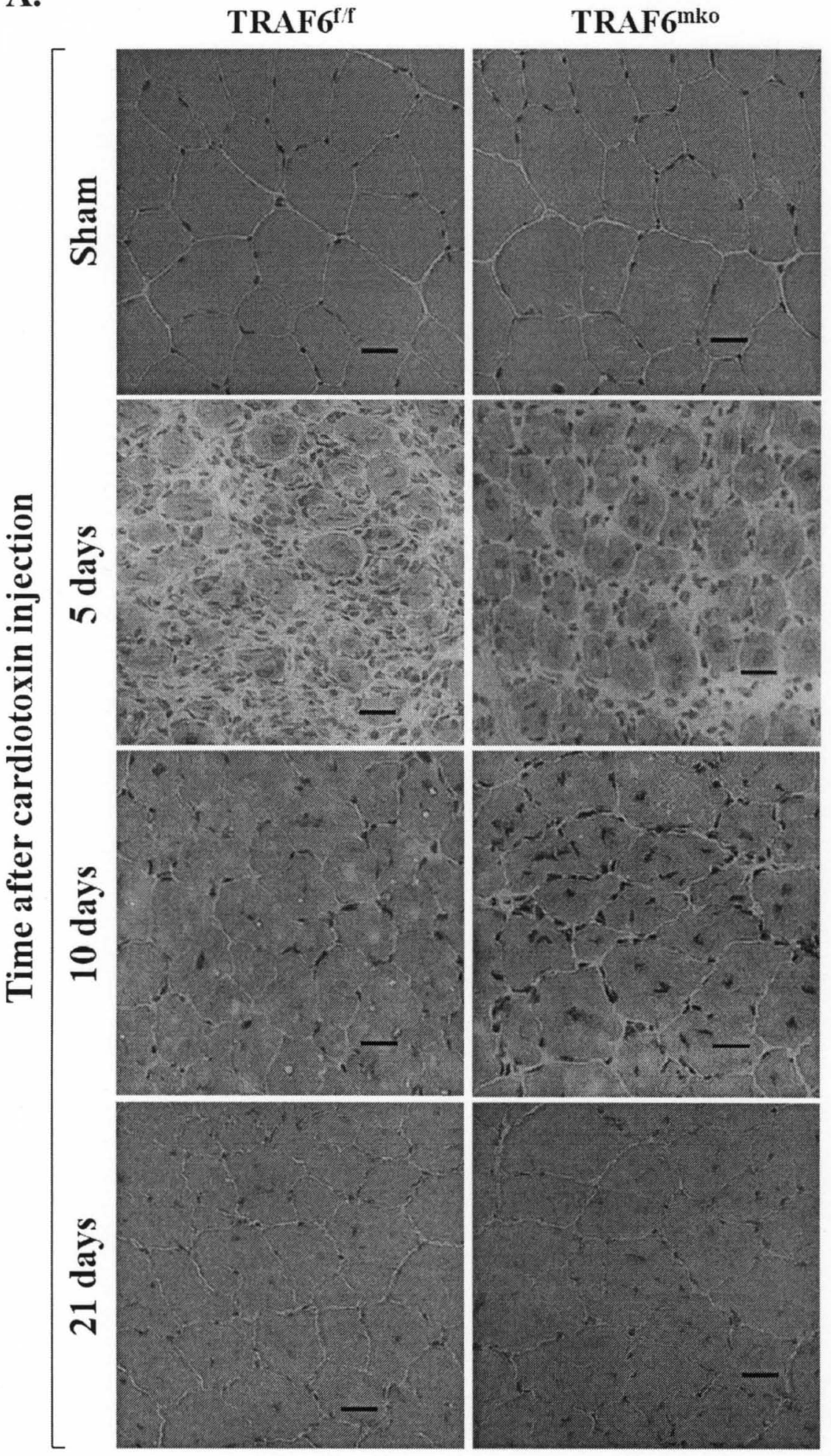




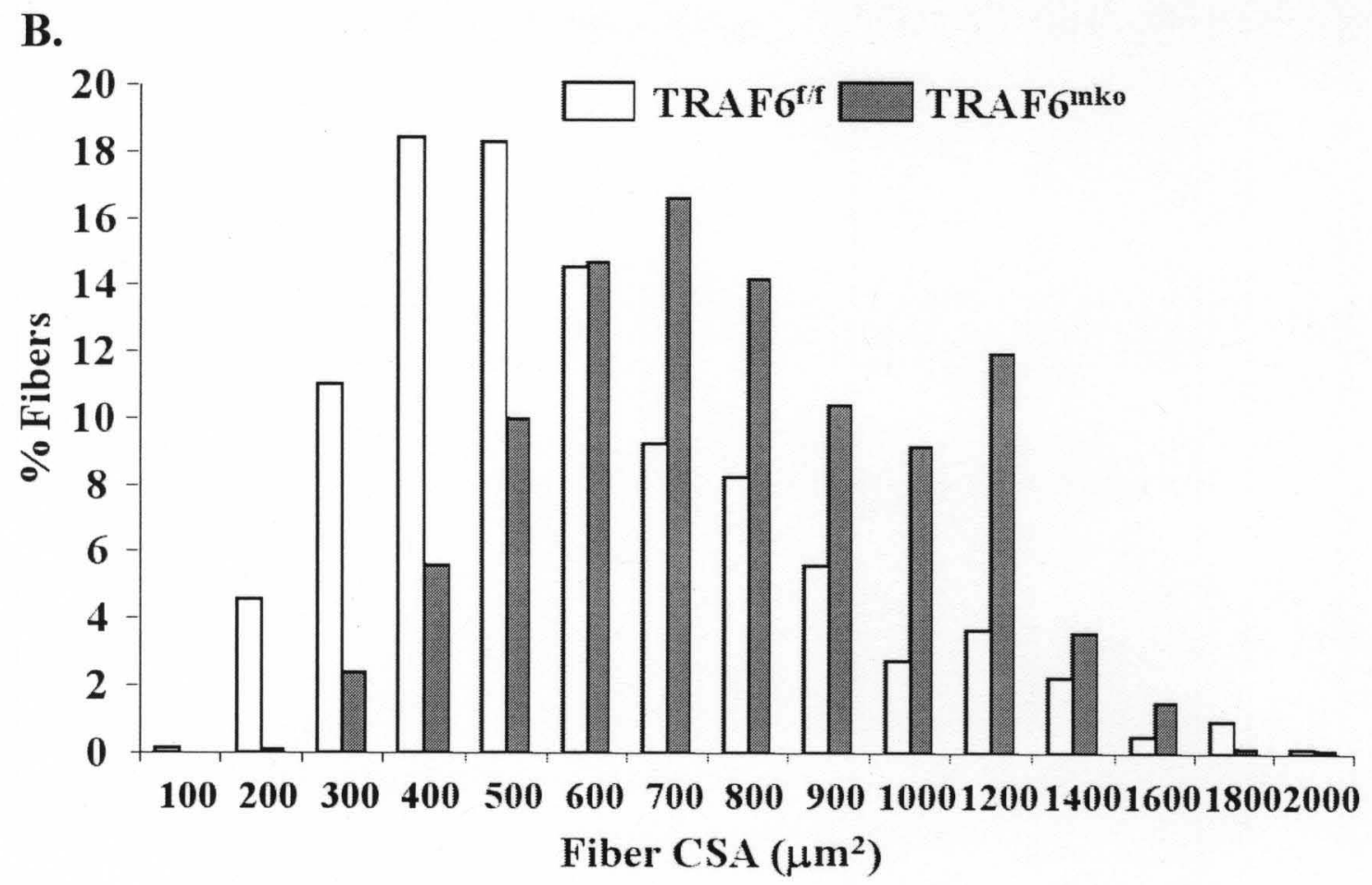

C.

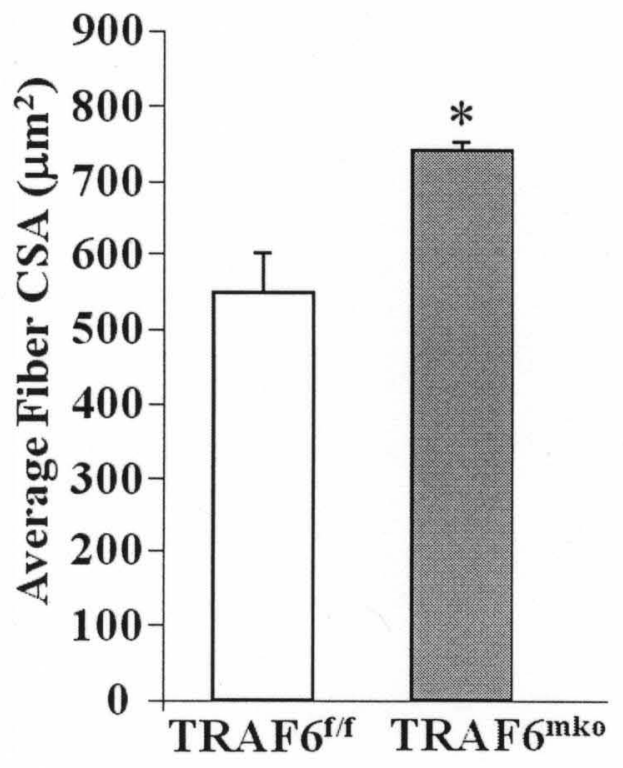

D.

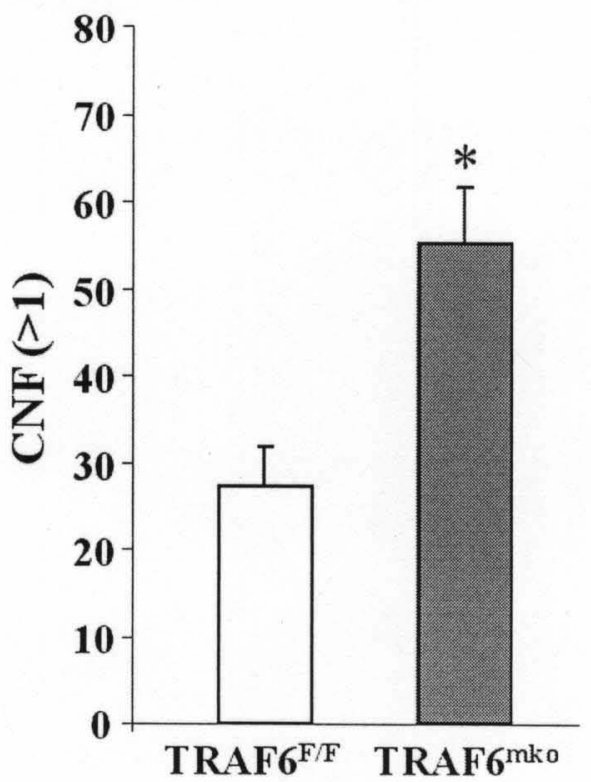


Figure 4.2: TRAF6 depletion accelerates post-injury regeneration in muscle. TRAF $^{\mathrm{f} / \mathrm{f}}$ and $\mathrm{TRAF}^{\mathrm{mko}}$ mice were injected either with saline or cardiotoxin in TA muscle and regenerating muscle were isolated and analyzed at different time points. (A) Representative micrographs of H\&E stained transverse sections of sham or Ctx-injured Tibialis anterior muscle at different time points. Scale bar: $20 \mu \mathrm{m}$ (B) Frequency distribution histograms representing cross-sectional area (CSA) of fibers in TA muscle from ctx-injured $\mathrm{TRAF}^{\mathrm{f} / \mathrm{f}}$ (open bars) and TRAF6 ${ }^{\mathrm{mko}}$ (shaded bars) at day 5 after injury (C) Mean CSA of myofibers in TA muscle from ctx-injured TRAF6 ${ }^{\mathrm{f} / \mathrm{f}}$ (open bar) and TRAF $^{\text {mko }}$ (shaded bar) at day 5 after injury. (D) Quantification of centrally nucleated fibers (CNF) at day 5 after ctx-injury in TA muscle from TRAF6 ${ }^{\mathrm{f} / \mathrm{f}}$ (open bar) and TRAF $6{ }^{\text {mko }}$ (shaded bar). Error bars represent SD. $N=4$ in each group. ${ }^{*} p<0.01$, values significantly different from Ctx-injured TA muscle of TRAF6 ${ }^{\mathrm{f} / \mathrm{f}}$. 
A.

TRAF6 $^{\mathrm{f} / \mathrm{f}}$

TRAF6 $^{\text {mko }}$
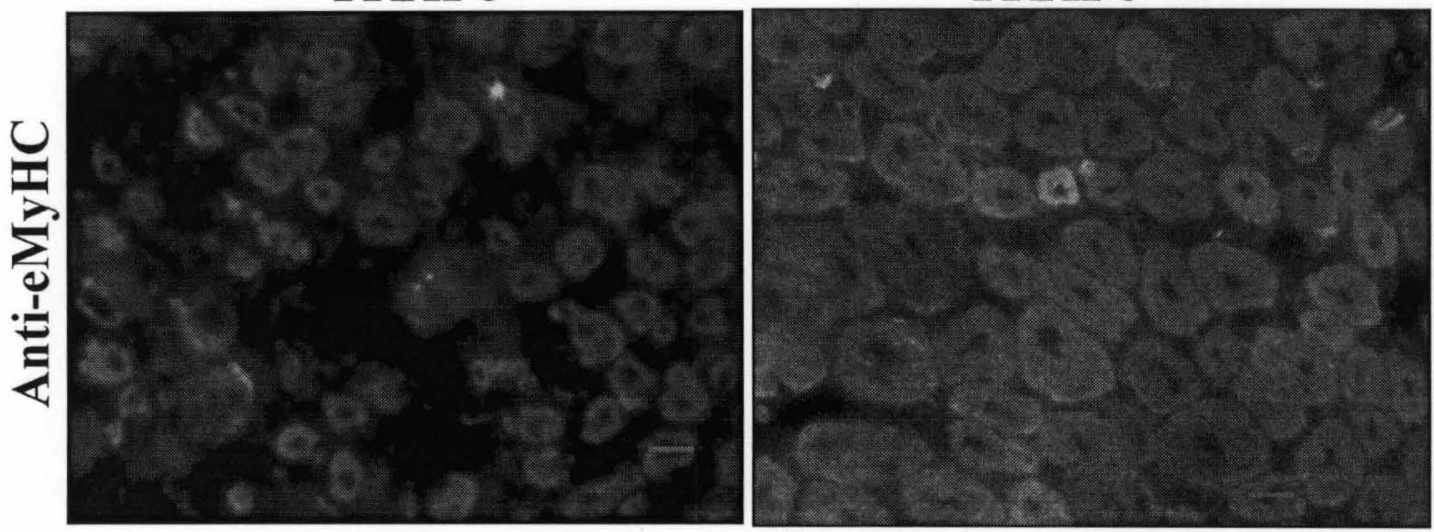

B.

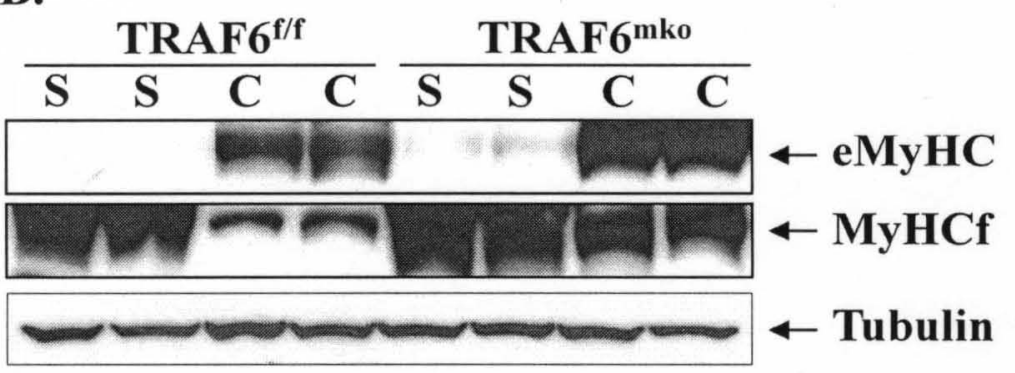

C.

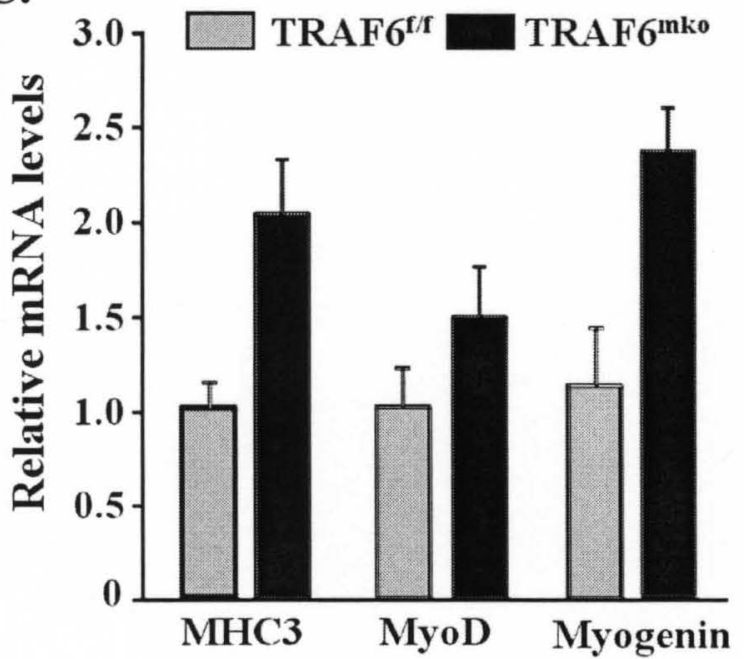


Figure 4.3: Early restoration of myoarchitecture in TRAF6-depleted skeletal muscle. Three month old TRAF $6^{\mathrm{f} / \mathrm{f}}$ and $\mathrm{TRAF} 6^{\mathrm{mko}}$ mice were injected with saline or Ctx in TA muscle and analyzed at day 5 after injury. (A) Transverse sections of Ctx-injured TA muscle from $\mathrm{TRAF}^{\mathrm{f} / \mathrm{f}}$ and $\mathrm{TRAF} 6^{\text {mko }}$ mice immunostained against embryonic isoform of myosin heavy chain (eMyHC). Scale bar: $20 \mu \mathrm{m}$. (B) Western blot analysis of expression levels of eMyHC or MyHc protein in sham or Ctx-injured TA muscle from TRAF $^{\mathrm{f} / \mathrm{f}}$ and TRAF6 ${ }^{\mathrm{mko}}$ mice. (C) Transcript levels of MHC3, MyoD and myogenin in sham or Ctx-injured TA muscle from TRAF $6^{\mathrm{f} / \mathrm{f}}$ and TRAF6 ${ }^{\mathrm{mko}}$ mice Error bars represent SD. $\mathrm{N}=4$ in each group. ${ }^{*} \mathrm{p}<0.01$, values significantly different from Ctx-injured TA muscle of TRAF $6^{\mathrm{f} / \mathrm{f}}$. 
A.
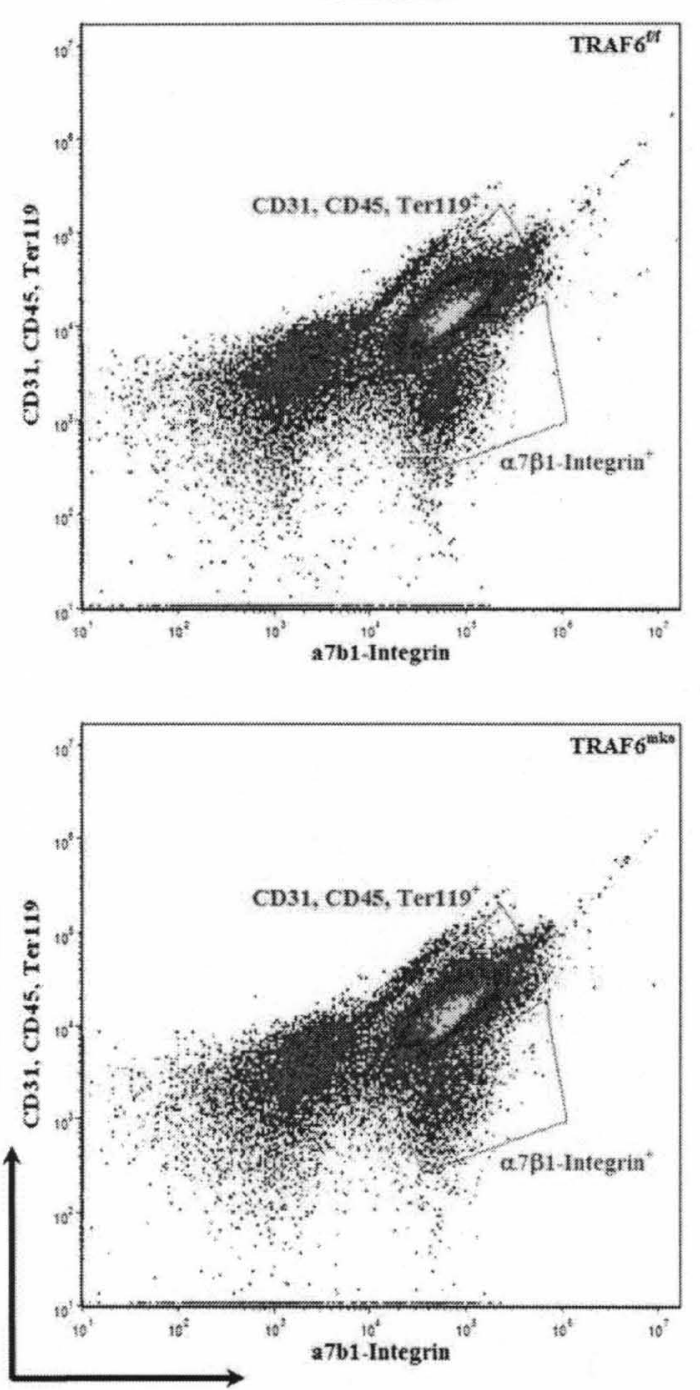

CTX-Injected
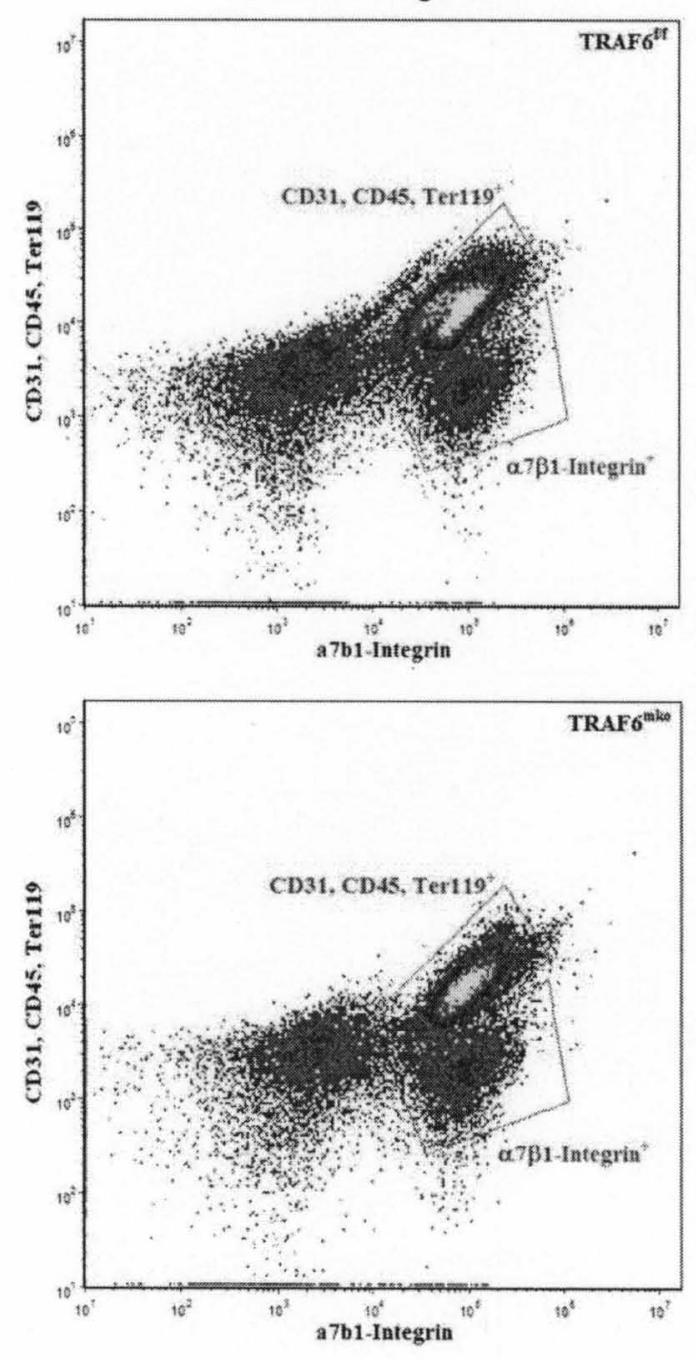
B.

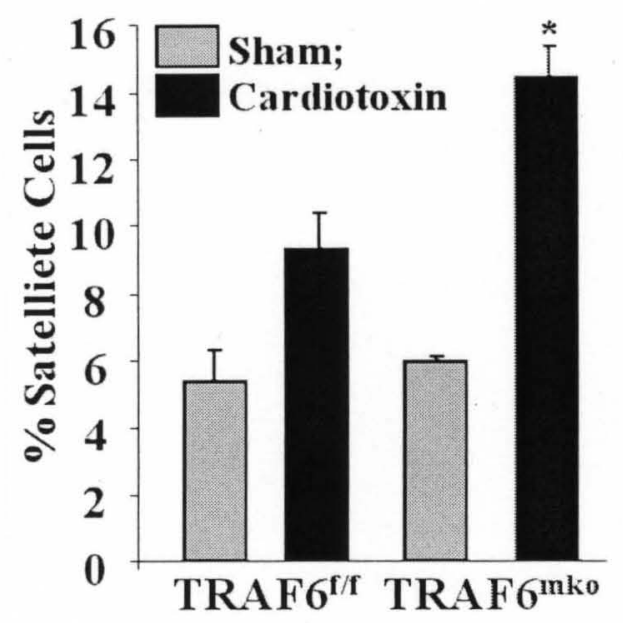

C.

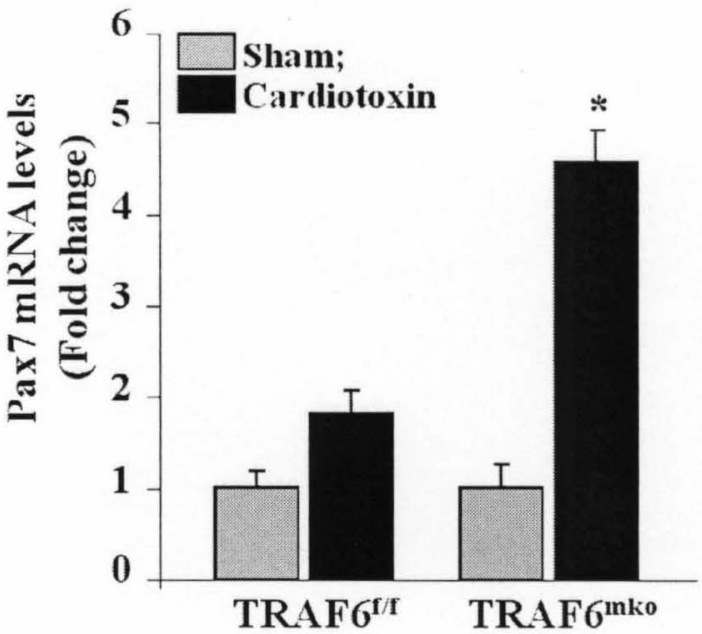

Figure 4.4: TRAF6 ablation promotes satellite cell activation during myogenesis in regenerating muscle. Three month old $\mathrm{TRAF}^{\mathrm{f} / \mathrm{f}}, \mathrm{TRAF}^{\mathrm{mko}}$ and $\mathrm{TRAF} 6^{\mathrm{dmko}}$ mice were injected with saline or Ctx in TA muscle and analyzed at day 5 after injury. (A) FACS analysis of saline or Ctx-injured TA muscle for $\alpha 7 \beta 1$-Integrin-positive activated satellite cells in TRAF6 ${ }^{\mathrm{f} / \mathrm{f}}$ and TRAF6 ${ }^{\mathrm{mko}}$ mice. (B) Quantification of activated satellite cells in sham or Ctx-injured TA muscles from TRAF6 ${ }^{\mathrm{f} / \mathrm{f}}$ and TRAF6 ${ }^{\mathrm{mko}}$ mice. (C) Transcript levels of Pax7 in sham or Ctx-injured TA muscle from TRAF6 ${ }^{\mathrm{f} / \mathrm{f}}$ and TRAF6 ${ }^{\mathrm{mko}}$ mice. Negative selection antibodies (CD45, CD31 and Ter119) gated in red, positive selection antibody ( $\alpha 7 \beta 1$-Integrin) gated in green. Error bars represent SD. $\mathrm{N}=4$ in each group. ${ }^{*} \mathrm{p}<$ 0.01 , values significantly different from Ctx-injured TA muscle of TRAF $6^{\mathrm{f} / \mathrm{f}}$. 
A.
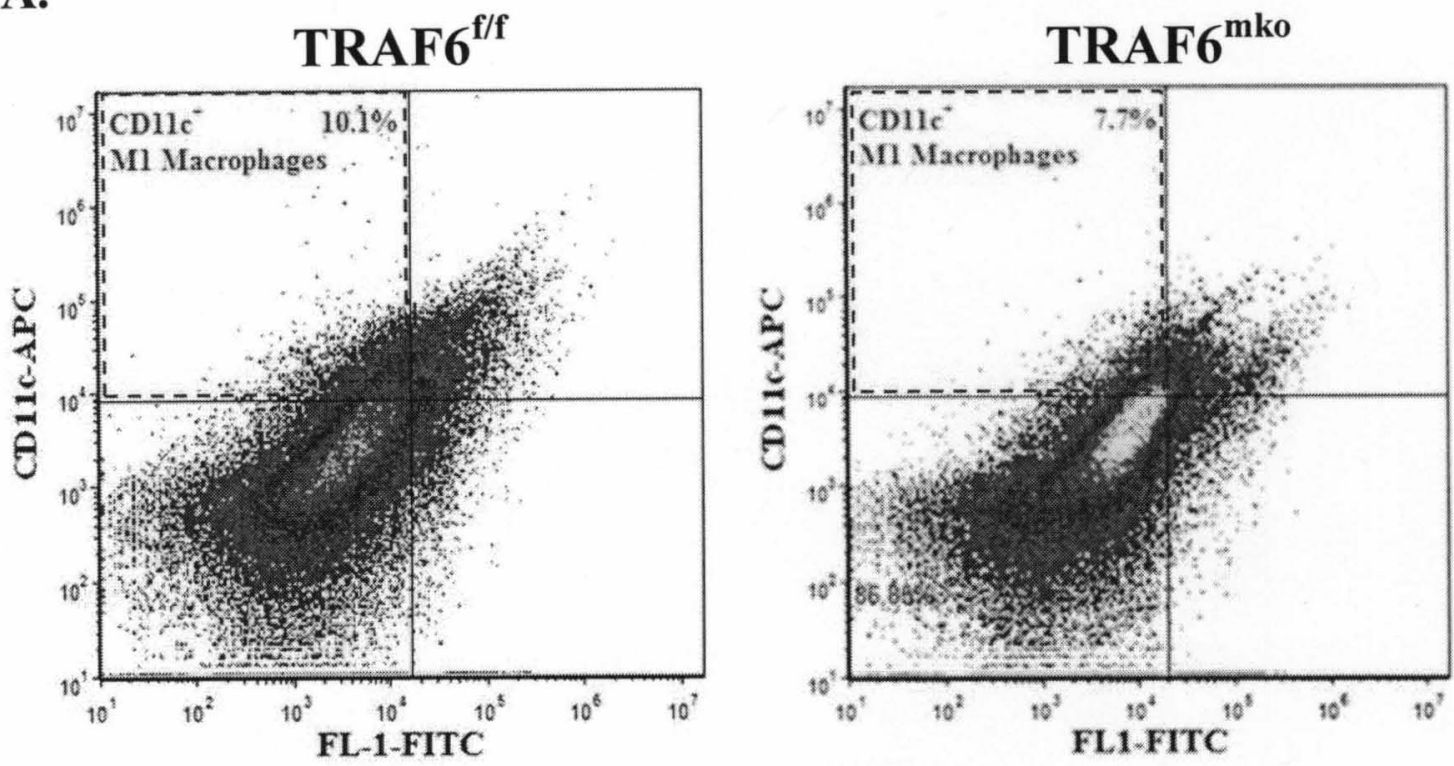

\section{M1 macrophages}
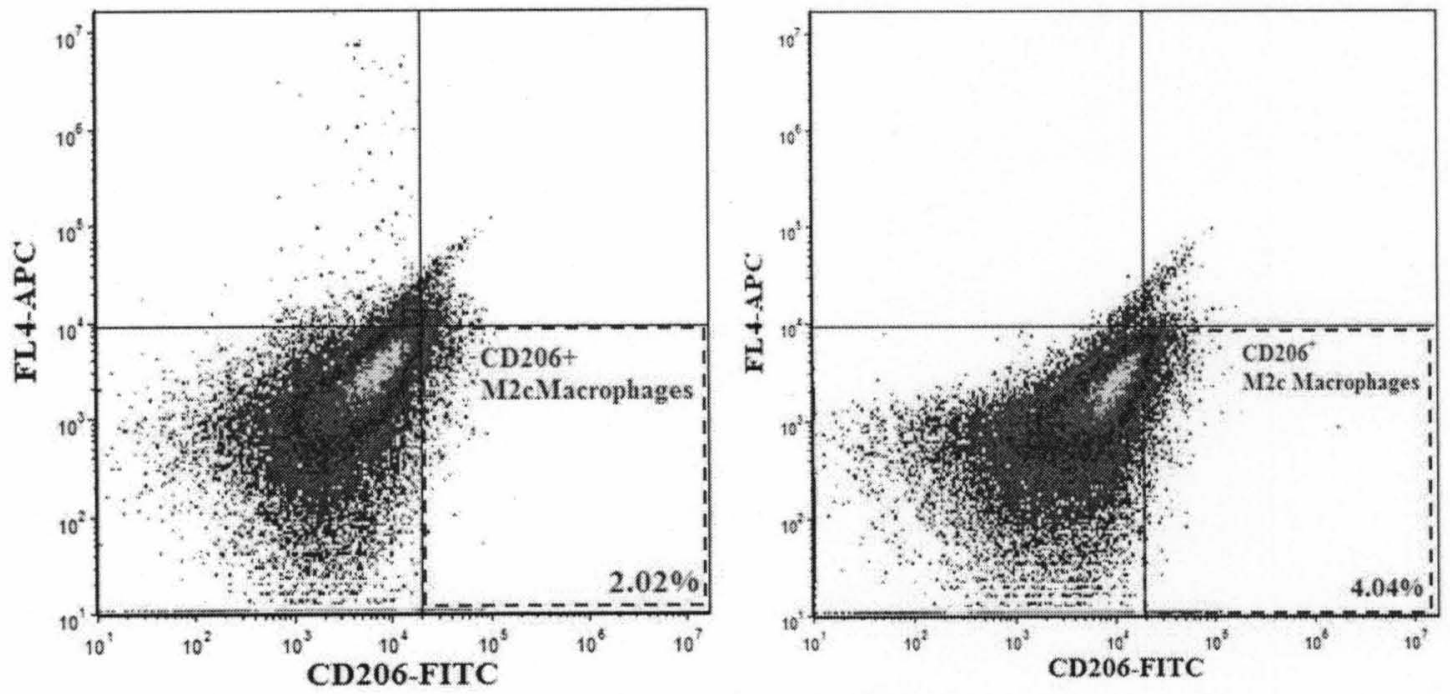

M2c macrophages 
B.

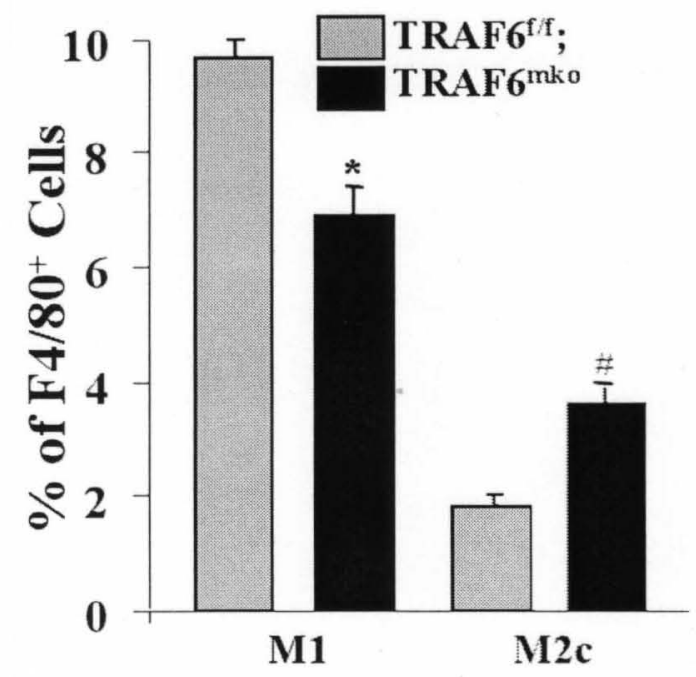

C.

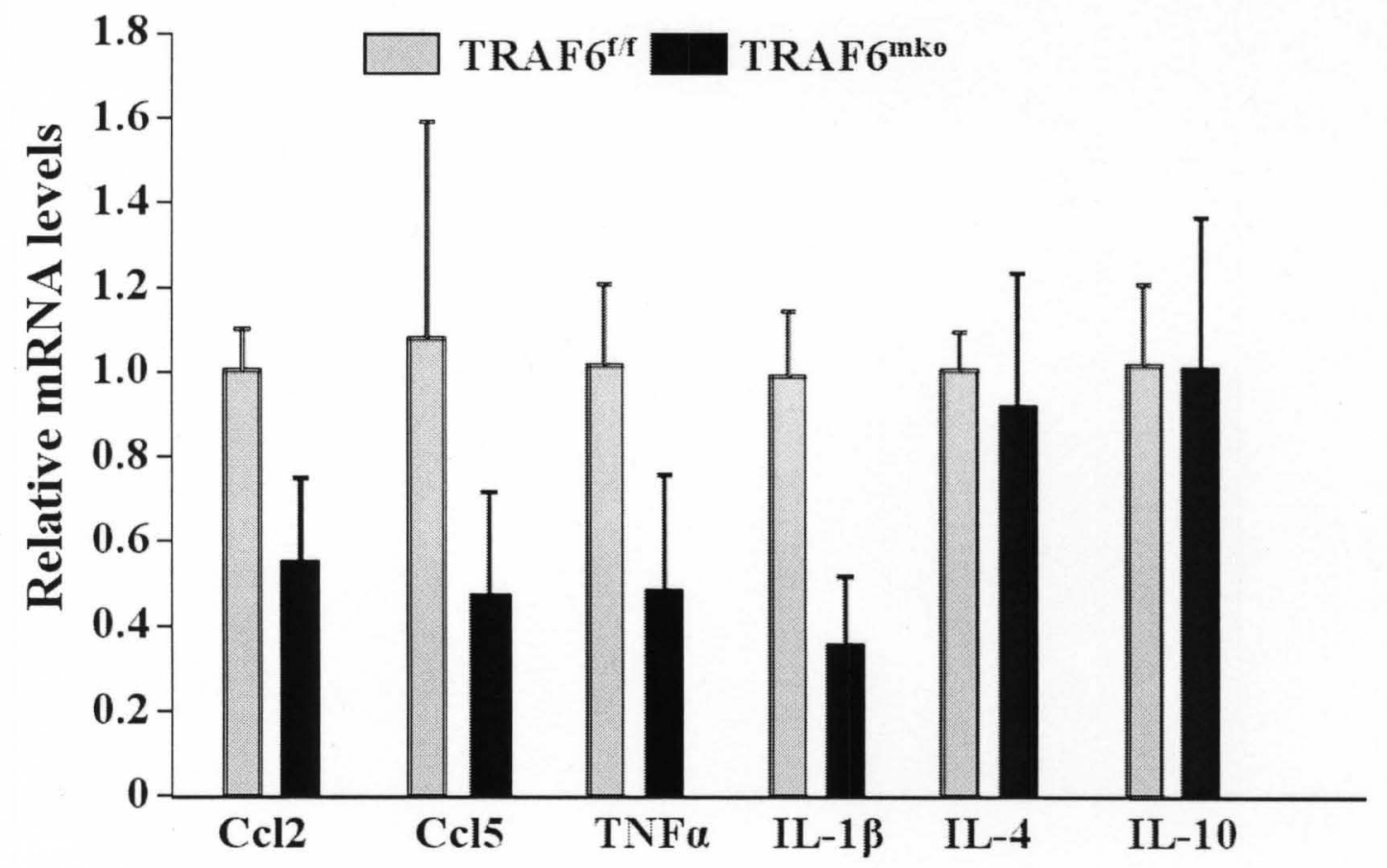


Figure 4.5: TRAF6 ablation promotes satellite cell activation during secondary myogenesis. Three month old TRAF $6^{\mathrm{f} / \mathrm{f}}, \mathrm{TRAF}^{\mathrm{mko}}$ and TRAF6 ${ }^{\mathrm{dmko}}$ mice were injected with saline or Ctx in TA muscle and analyzed at day 5 after injury. (A) FACS analysis for M1 (CD11 $\mathrm{c}^{+}$) and M2 (CD206 ${ }^{+}$) macrophage phenotypes in Ctx-injured muscles of TRAF6 ${ }^{\mathrm{f} / \mathrm{f}}$, TRAF6 ${ }^{\mathrm{mko}}$ and TRAF6 ${ }^{\mathrm{dmko}}$ mice. (B) Quantification of macrophage phenotype composition in regenerating TA muscle from TRAF $6^{\mathrm{f} / \mathrm{f}}, \mathrm{TRAF}^{\mathrm{mko}}$ and TRAF ${ }^{\text {dmko }}{ }^{\text {mice. }}$. (C) Transcript levels of proinflammatory TNF $\alpha$ and IL-1 $\beta$, IL4 and IL10 and CCL2 and $\mathrm{CCl} 5$ in regenerating from $\mathrm{TRAF}^{\mathrm{f} / \mathrm{f}}$ and TRAF ${ }^{\mathrm{dmko}}$ mice. M1 macrophages (CD11 ${ }^{+}$) are gated in red while M2 macrophages $\left(\mathrm{CD} 206^{+}\right)$are gated in green. Error bars represent SD. $N=6$ in each group. ${ }^{* / \#} \mathrm{p}<0.01$, values significantly different from Ctx-injured TA muscle of TRAF $6^{\mathrm{f} / \mathrm{f}}$. 

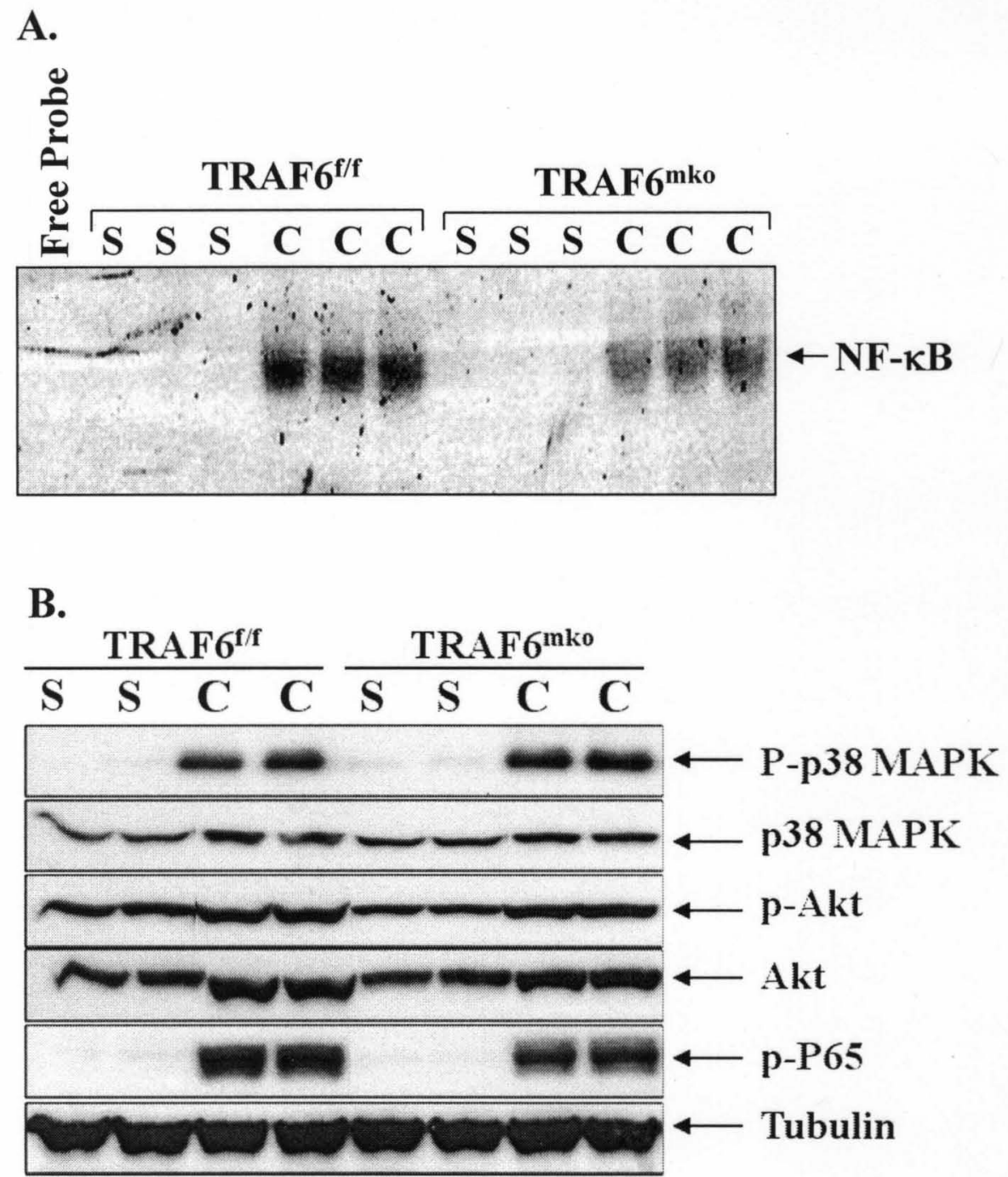

Figure 4.6: Effect of TRAF6 on signaling molecules in regenerating muscles. Three month old TRAF6 ${ }^{\mathrm{f} / \mathrm{f}}$, and TRAF6 ${ }^{\mathrm{mko}}$ mice were injected with saline or Ctx in TA muscle and analyzed at day 5 after injury. (A) Activation of NF- $\mathrm{kB}$ was analyzed in response to Ctx-injury in $\mathrm{TRAF}^{\mathrm{mko}}{ }^{\mathrm{m}}$ mice compared with $\mathrm{TRAF} 6^{\mathrm{f} / \mathrm{f}}$ mice. (B) Representative western blots of phospho-p38, total p38, phospho-Akt, total Akt, phospho-p65 (relA) and $\alpha$ tubulin in sham or Ctx-injured TA muscles of TRAF6 ${ }^{\text {mko }}$ mice compared with that of TRAF6 ${ }^{\mathrm{f} / \mathrm{f}}$ mice. 
A.

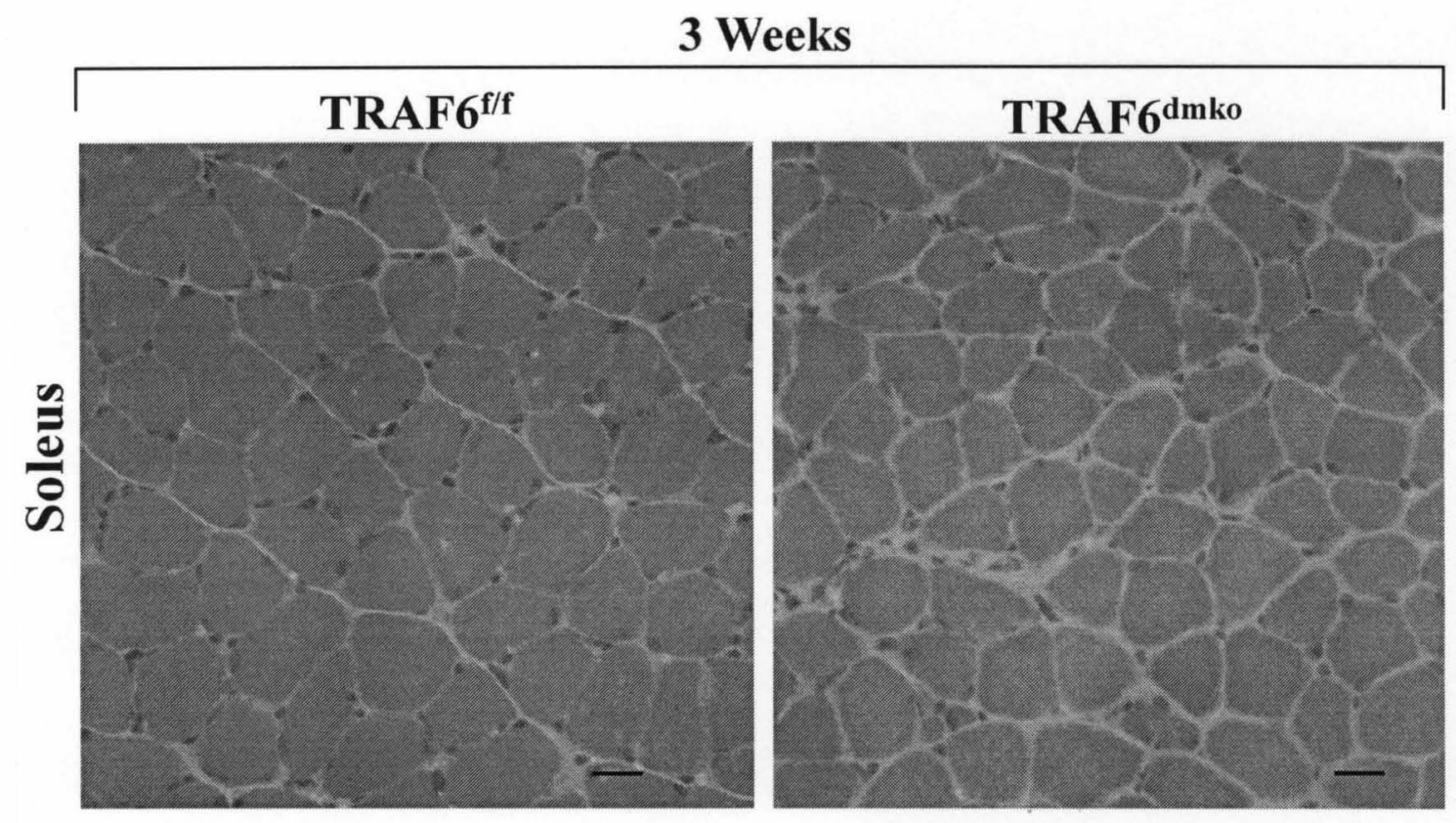

10 Weeks

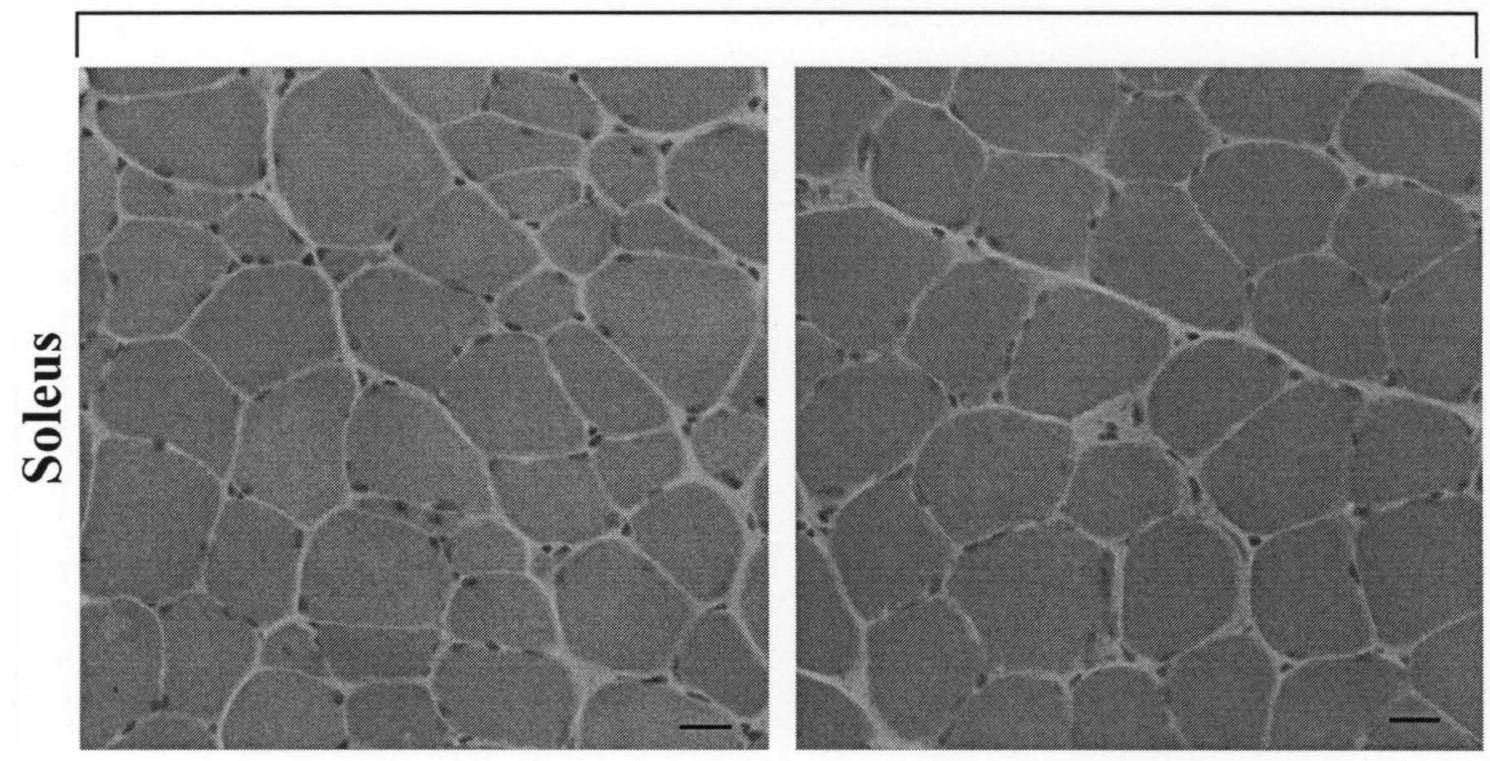



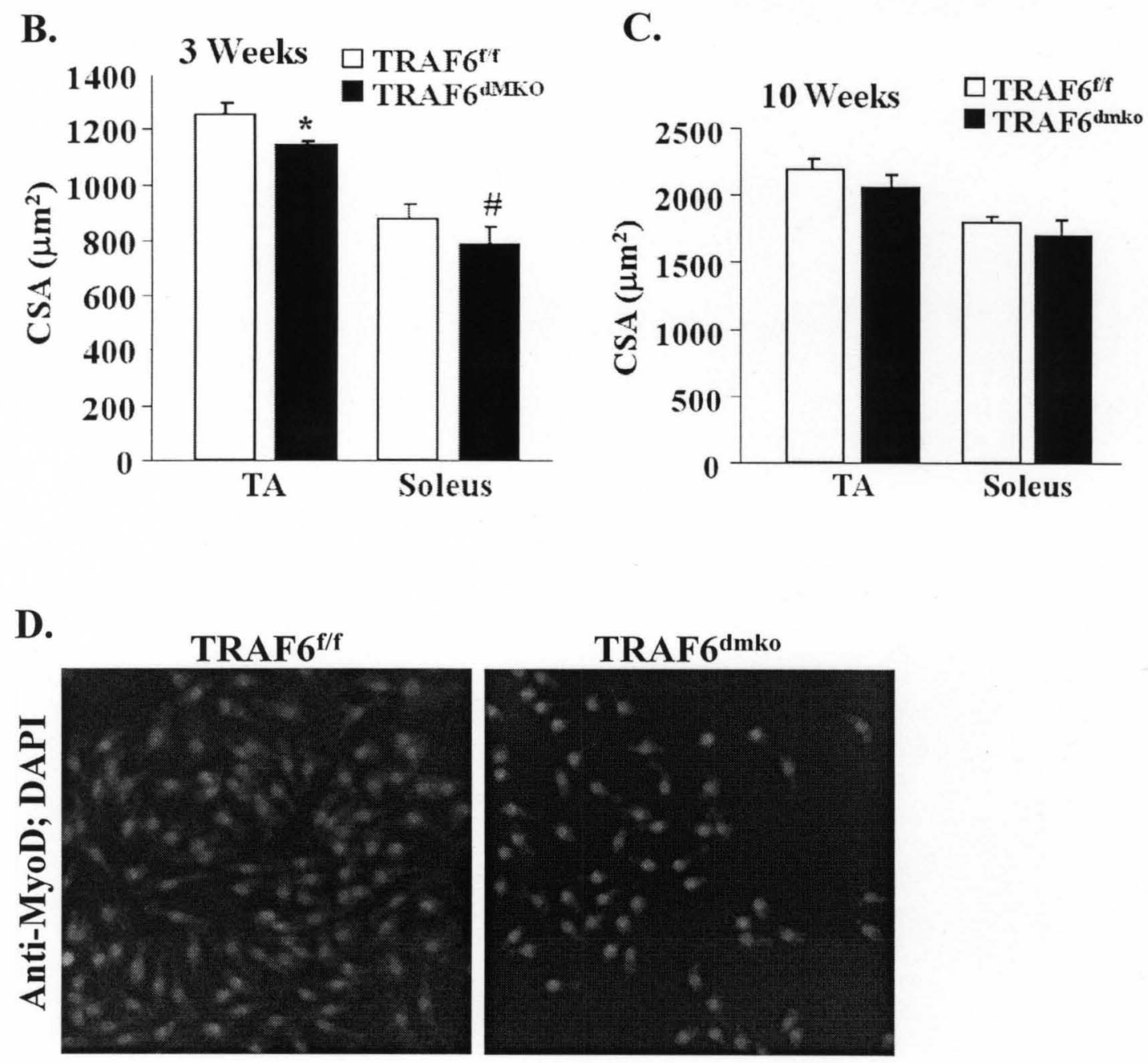

E.

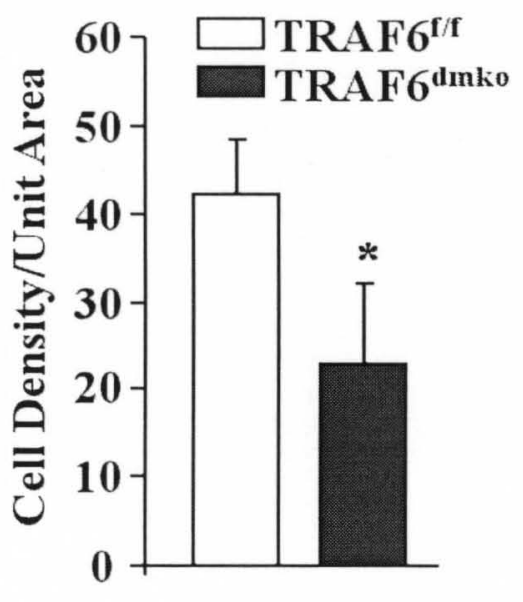

F.

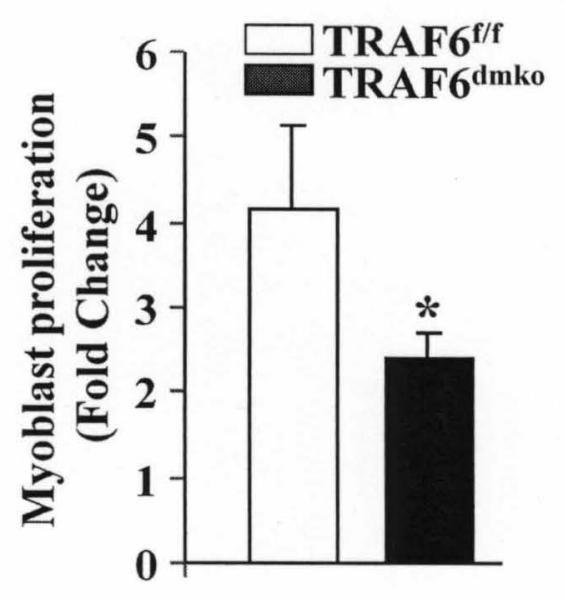


G.
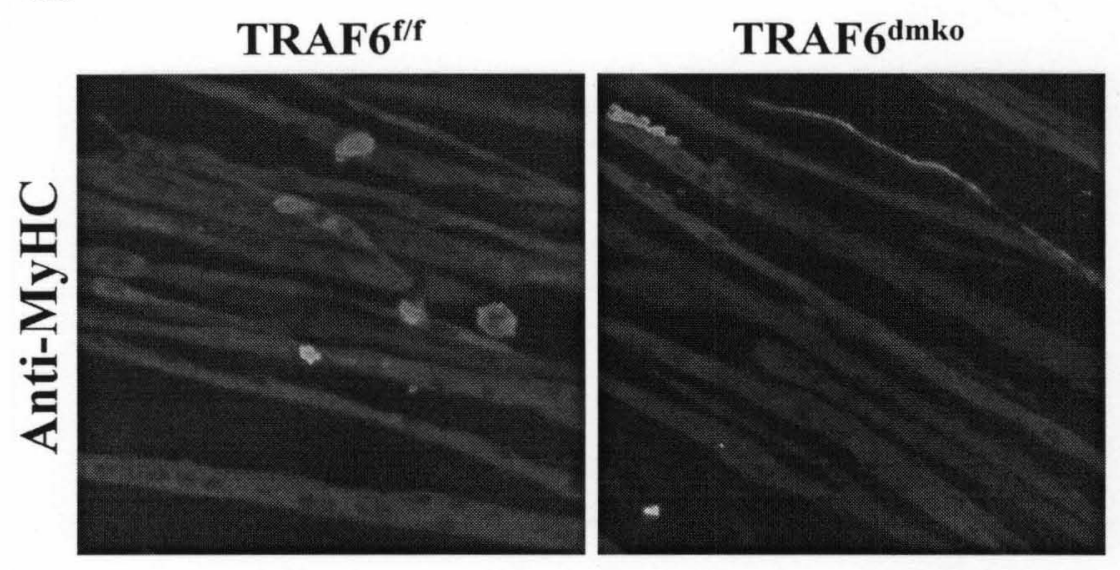

H.

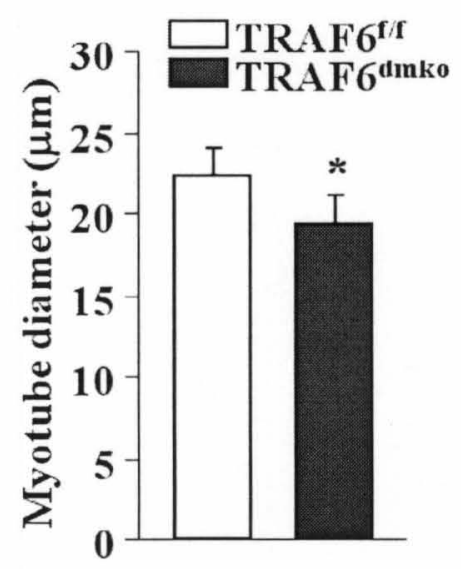

I.

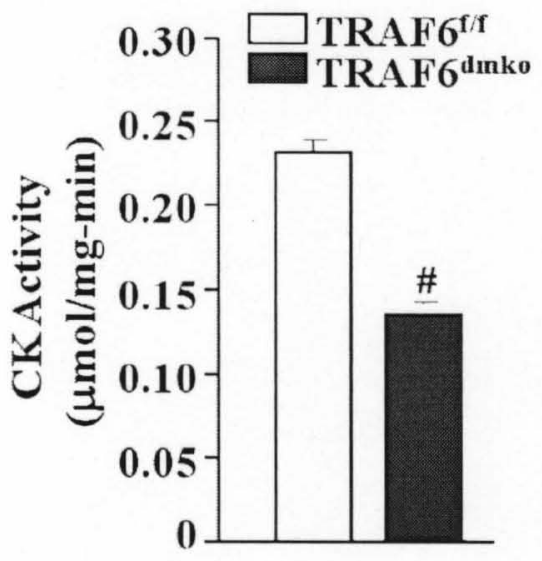

Figure 4.7: Depletion of TRAF6 early during embryonic development delays muscle growth. Three weeks or 10 weeks old TRAF $6^{\text {t/f }}$ and TRAF $6^{\text {dmko }}$ mice were analyzed by morphometric methods. (A) Representative photomicrographs of H\&E stained soleus muscle at $3 \mathrm{wk}$ and $10 \mathrm{wk}$ and (B) mean myofiber CSA demonstrate a delayed muscle formation in 3 week old TRAF6 ${ }^{\mathrm{dmko}}$ mice compared to TRAF6 ${ }^{\mathrm{fff}}$ mice. (C) Average myofiber CSA from TA and soleus muscle from 10-week old TRAF6 $6^{\mathrm{f} / \mathrm{f}}$ and TRAF $6^{\text {dmko }}$ mice exhibit no significant difference. . (D) Cell proliferation efficiency was analyzed in myoblasts. TRAF6 ${ }^{\text {dmko }}$ mice demonstrated significantly attenuated cell proliferation efficiency. (E) and (F) Reduced cell proliferation in primary myoblasts from TRAF $6^{\text {dmko }}$ compared to $\mathrm{TRAF}^{\mathrm{f} / \mathrm{f}}$ as demonstrated by cell density/unit area and cell proliferation efficiency. (G) Reduced myotube formation in TRAF6 ${ }^{\text {dmko }}$ mice $(\mathbf{H})$ and (I) Attenuated muscle formation in TRAF $6^{\mathrm{dmko}}$ mice as compared to $\mathrm{TRAF}^{\mathrm{f} / \mathrm{f}}$ mice demonstrated by smaller myofiber diameter and reduced creatine kinase activity. Scale bars: $20 \mu \mathrm{m}$. Error bars represent SD. $\mathrm{N}=8$ in each group. ${ }^{* / \#} \mathrm{p}<0.01$, values significantly different from TA or soleus muscle of TRAF $6^{\mathrm{f} / \mathrm{f}}$. 
A.

Time after cardiotoxin injection
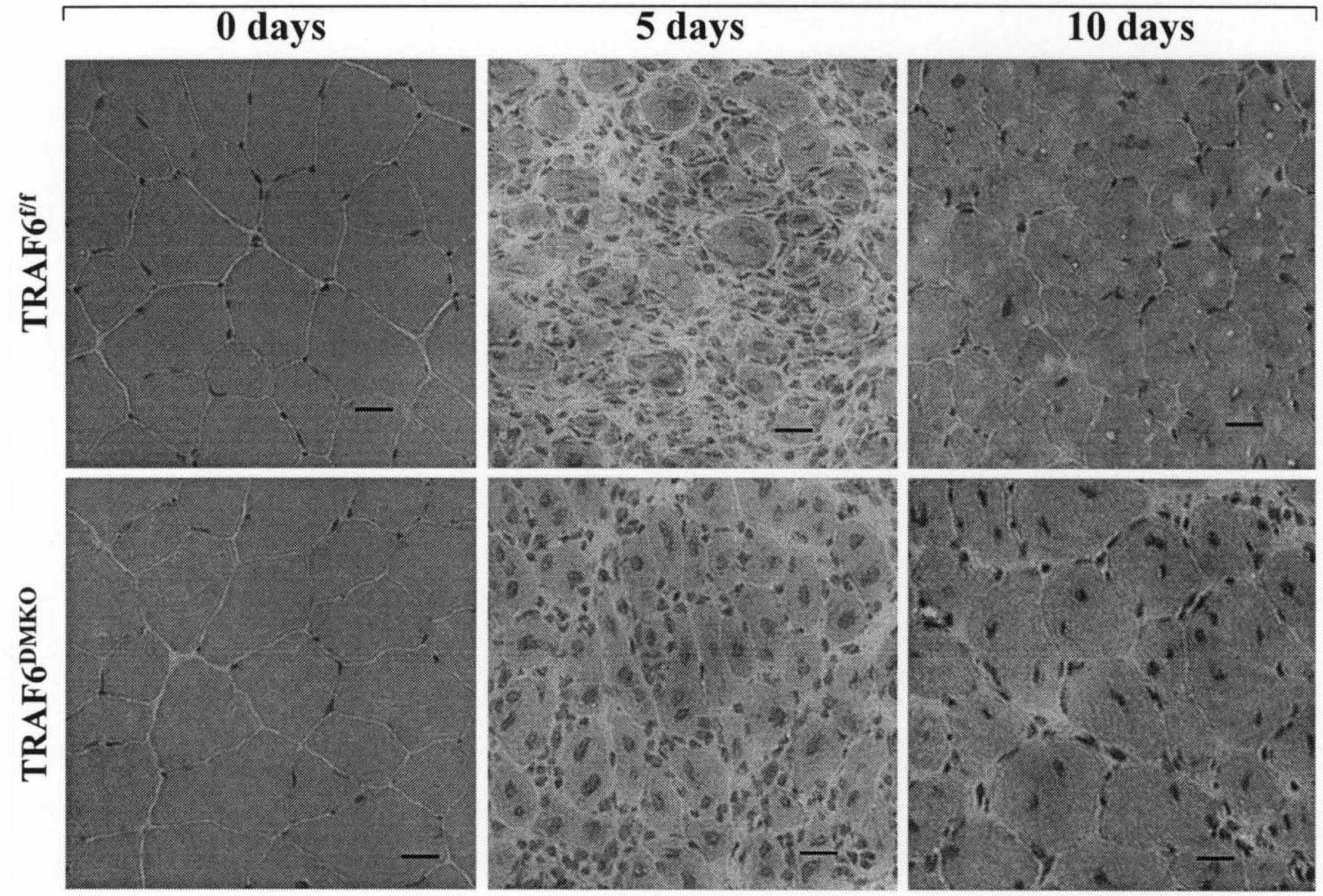

B.

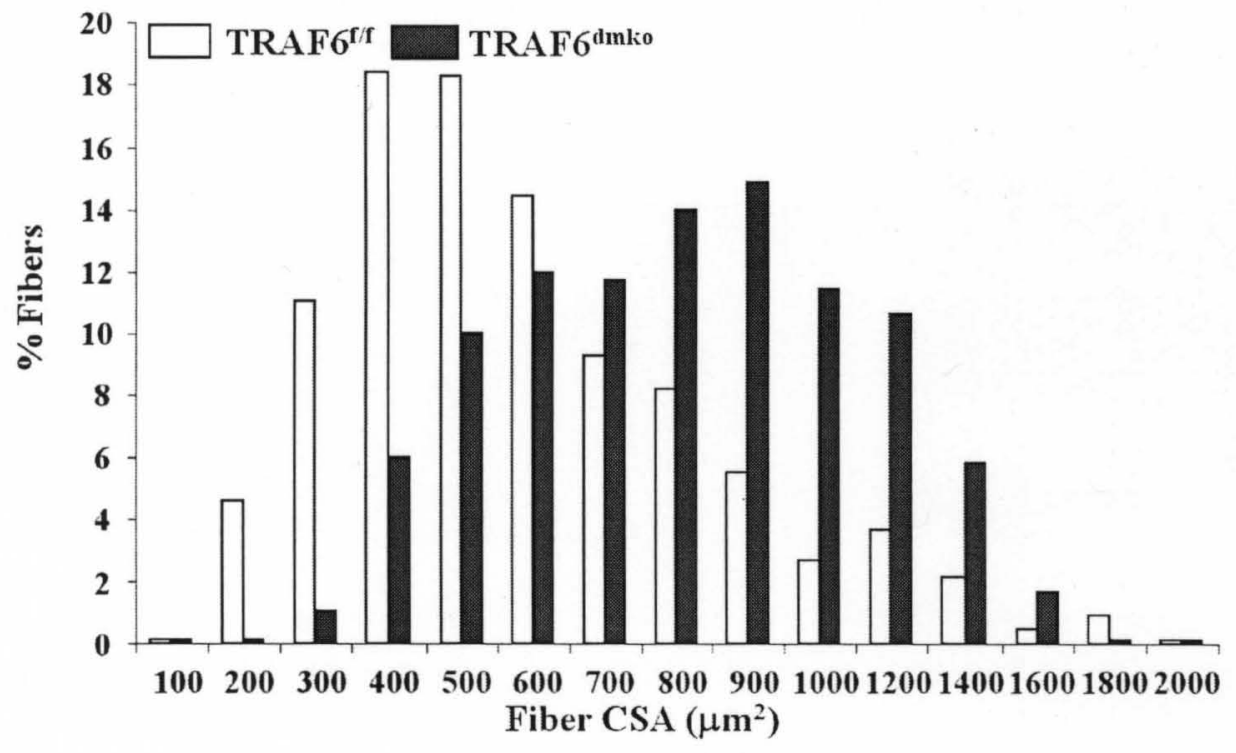


C.

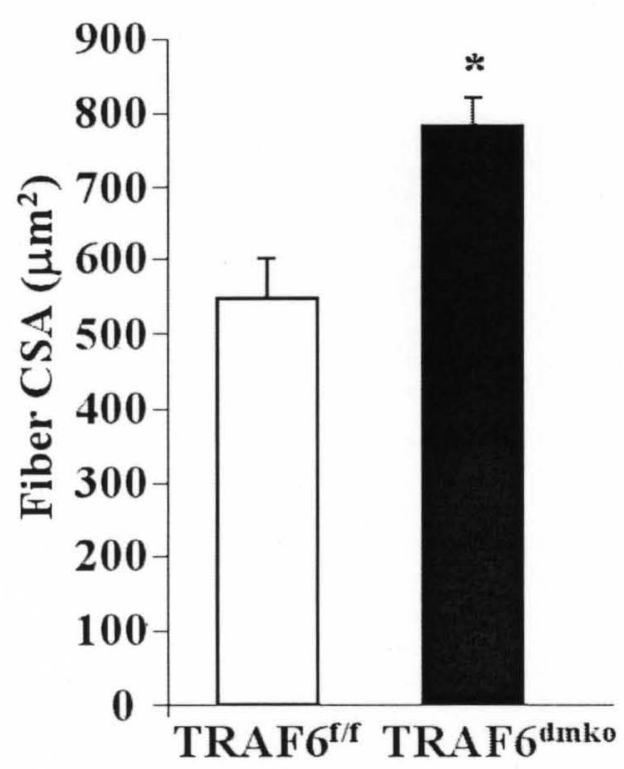

E.

TRAF6 $^{\text {fif }}$

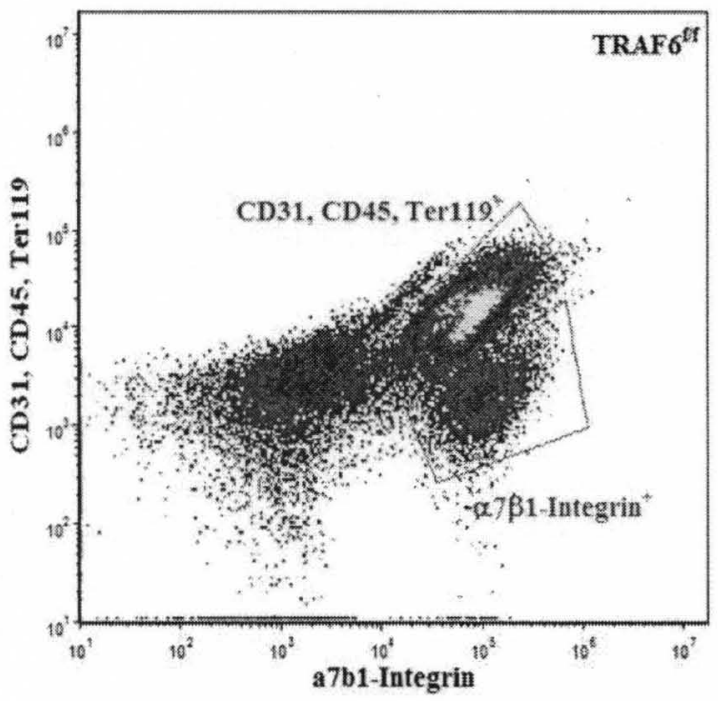

D.

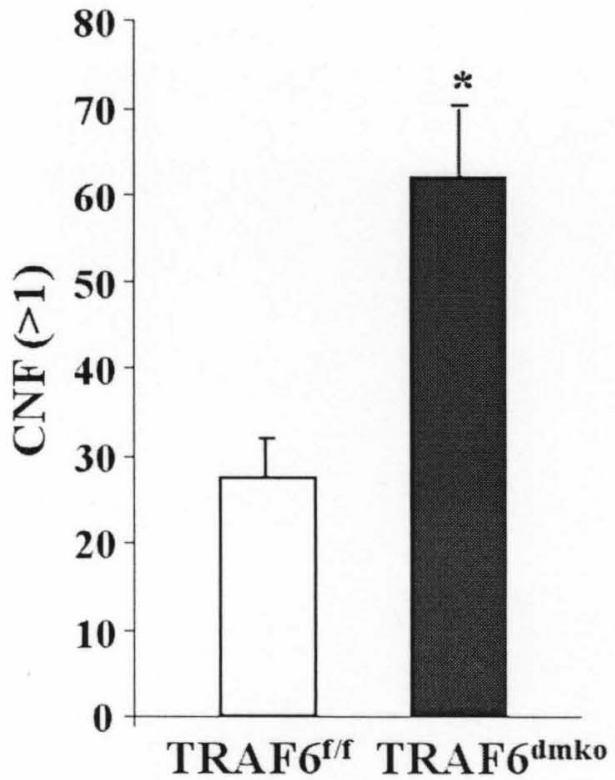

TRAF6 ${ }^{\text {dmko }}$

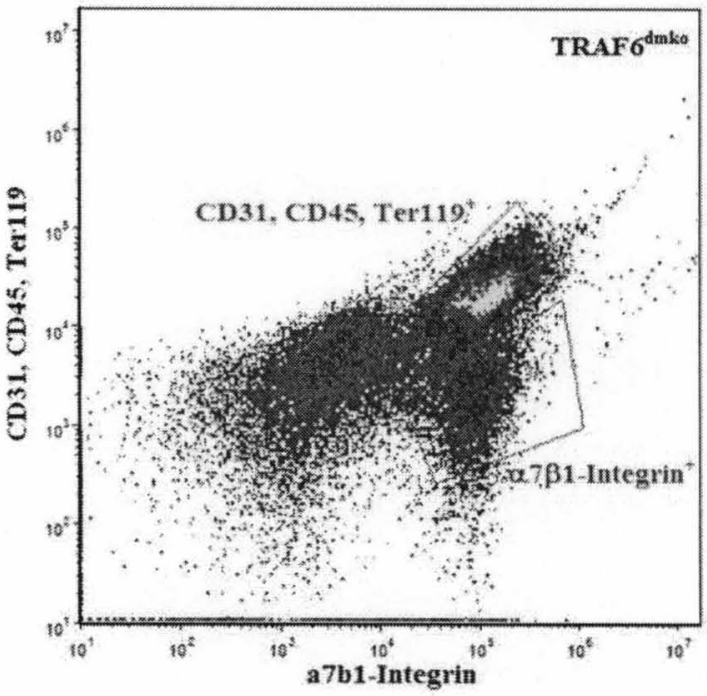


F.

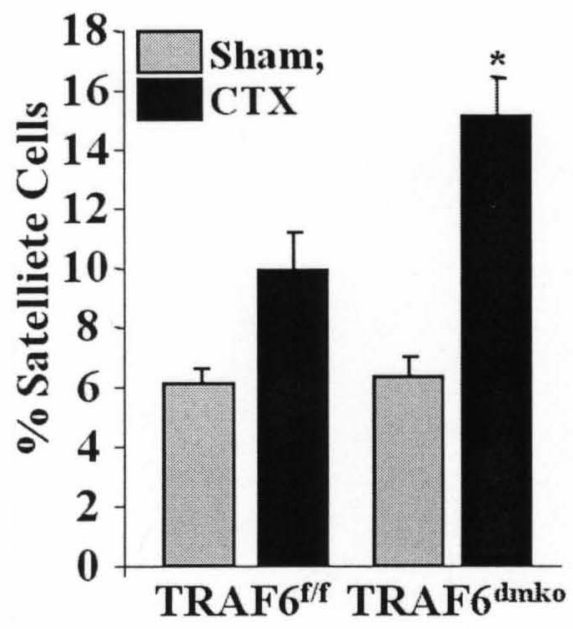

G.

M1 Macrophages
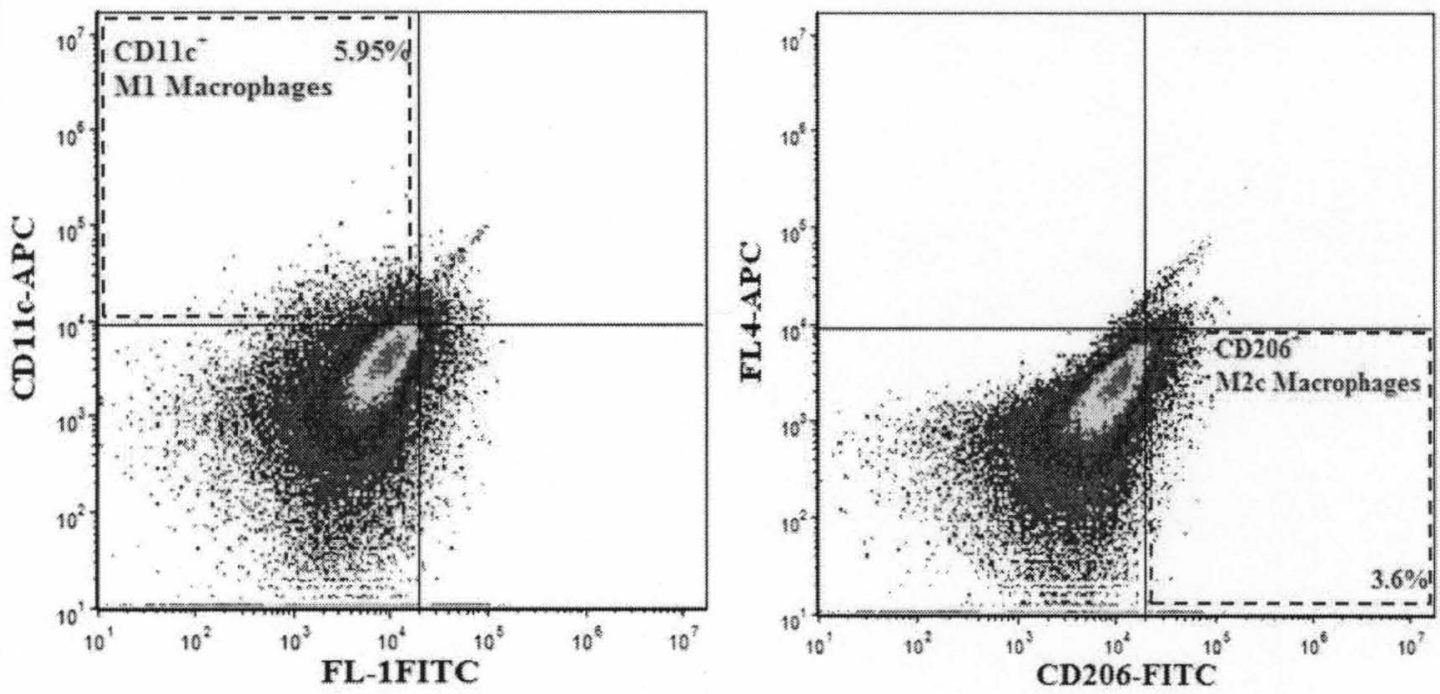


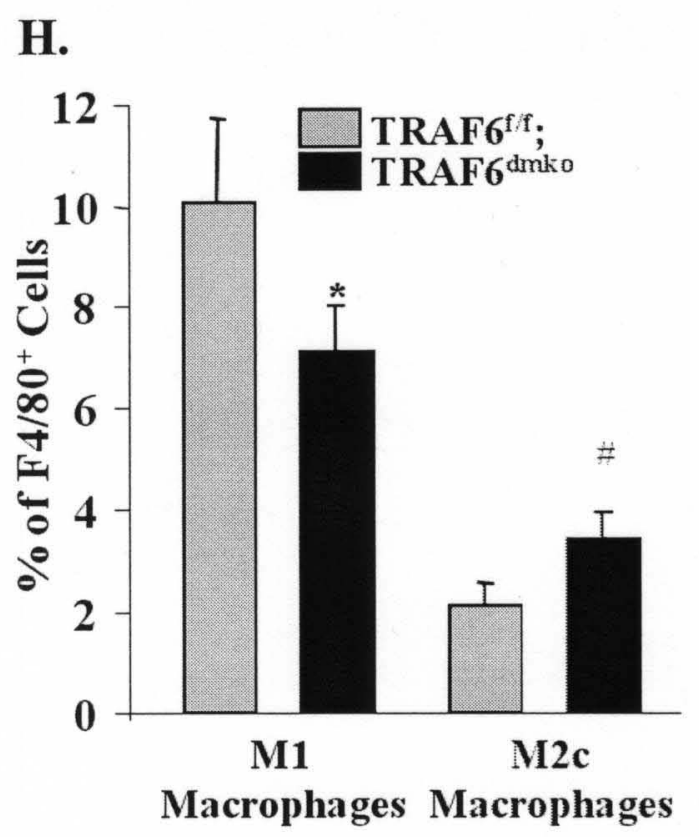

Figure 4.8: Depletion of TRAF6 early during embryonic development improves regeneration. Three month old TRAF $6^{\mathrm{f} / \mathrm{f}}$ and TRAF $6^{\text {dmko }}$ mice were injected either with saline or cardiotoxin in TA muscle and regenerating muscle were isolated and analyzed at different time points. (A) Representative micrographs of H\&E stained transverse sections of sham or Ctx-injured Tibialis anterior muscle at different time points demonstrate a significantly improved regeneration in TRAF $6^{\text {dmko }}$ mice compared to TRAF $6^{\mathrm{f} / \mathrm{f}}$ control. Scale bar: $20 \mu \mathrm{m}$ (B) and (C) Quantification of distribution of fibers by CSA and mean CSA of Ctx-injured TA muscle from TRAF6 $6^{\mathrm{f} / \mathrm{f}}$ and TRAF6 ${ }^{\mathrm{dmko}}$ mice. (D) Number of centrally nucleated fibers were significantly higher in TRAF $6^{\text {dmko }}$ showing an accelerated regeneration. Developmental knock-out of TRAF6 improves regeneration. TRAF6 ${ }^{\text {f/f }}$ and TRAF $6^{\text {dmko }}$ mice were injected either with saline or cardiotoxin in TA muscle and regenerating muscle were isolated and analyzed at different time points. (E) FACS analysis of saline or Ctx-injured TA muscle for $\alpha 7 \beta 1$-Integrin-positive activated satellite cells in TRAF6 ${ }^{\mathrm{f} / \mathrm{f}}$ and TRAF6 ${ }^{\text {dmko }}$ mice. (F) Quantification of activated satellite cells in sham or Ctx-injured TA muscles from $\mathrm{TRAF}^{\mathrm{f} / \mathrm{f}}$ and $\mathrm{TRAF} 6^{\mathrm{dmko}}$ mice. (G) FACS analysis for M1 $\left(\mathrm{CD} 11 \mathrm{c}^{+}\right)$and $\mathrm{M} 2\left(\mathrm{CD} 206^{+}\right)$macrophage phenotypes in Ctx-injured muscles of TRAF6 $6^{\mathrm{f} / \mathrm{f}}$ and TRAF6 ${ }^{\mathrm{dmko}}$ mice. (D) Quantification of macrophage phenotype composition in regenerating TA muscle from TRAF $6^{\mathrm{f} / \mathrm{f}}$ and TRAF6 ${ }^{\mathrm{dmko}}$ mice. Scale bars: $20 \mu \mathrm{m}$. Error bars represent SD. N=8 in each group. ${ }^{* / \#} \mathrm{p}<0.01$, values significantly different from Ctx-injured TA muscle of TRAF $6^{\text {fif }}$. 


\section{CHAPTER FIVE}

\section{CONCLUSION AND FUTURE WORK}

This chapter summarizes the work presented in this dissertation and discusses its implications in understanding skeletal muscle remodeling. It also highlights the contribution of this research for scholars in the field of skeletal muscle biology. The discussion concludes by acknowledging some of the research limitations and suggesting areas for future work.

\subsection{Review of Dissertation and Skeletal Muscle Remodeling}

Skeletal muscle remodeling is an extremely complex response to a number of catabolic cues $(3,117,147,150,182-184)$. These signaling cues stimulate activation of several intracellular pathways which eventually lead to transient or persistent remodeling of skeletal muscle. Although our knowledge about intracellular mechanisms in skeletal muscle atrophy or regeneration has increased multifold in recent years, it largely remains

unknown that how proximal signaling events stimulate the activation of crucial downstream cascades. This study has explored the role of an important upstream signaling molecule TRAF6 and has identified it as a novel regulator of skeletal muscle remodeling especially in cases of atrophy and injury-induced regeneration in skeletal muscle.

As I showed in Chapter 2, receptor-mediated activation of TRAF6 increases in response to catabolic signals. In turn, activated TRAF6 regulates skeletal muscle mass by 
activation and recruitment of proteasomal and lysosomal degradation pathways in response to denervation (loss of trophic support to muscle). TRAF6-mediated activation of UPS, ALS, NF- $\mathrm{BB}$, catabolic kinases and muscle specific E3 ubiquitin-ligases cumulatively lead to degradation of muscle proteins and result in altered muscle architecture and reduced contractile ability in skeletal muscle. In a murine model of muscle specific TRAF6 knock-out (TRAF6 ${ }^{\text {mko }}$ ) used in this study, it has been shown that depletion of TRAF6 repressed the activation of several of the major catabolic signaling mechanisms and their components in denervation-induced atrophy. This study also shows that ablation of TRAF6 significantly rescues the normal mitochondrial organization and distribution and prevents the formation of autophagosome in denervated skeletal muscle of mice. In addition to disuse (denervation-induced) atrophy, this study also investigated the role of TRAF6 in cancer cachexia-induced muscle loss. Similar to its effects in response to denervation, loss of TRAF6 significantly ameliorated muscle atrophy in cachectic conditions. Through inhibition of NF- $\mathrm{kB}$, muscle specific E3 ligases and autophagy-related genes, TRAF6 ablation significantly rescued loss of muscle mass in response to cancer cachexia.

The chapter 3 of this study discusses the role of TRAF6 in starvation-induced atrophy and identifies TRAF6 as a crucial regulator of atrophic response. In this study, I showed that TRAF6 is not only involved in canonical catabolic pathways common to all atrophies, but also exhibits distinct regulatory interventions in different models of atrophy. In starvation-induced atrophy, TRAF6 activates muscle specific E3 ligases, UPS and ALS, all of which are significantly inhibited in TRAF6 ablated skeletal muscle. The most salient finding of this study is identification of regulatory role of TRAF6 in 
activation of unfolded protein response (UPR) and endoplasmic reticulum (ER) stress mechanisms and the role of later two in skeletal muscle atrophy. All the three arms of UPR (GADD34, XBP-1 and ATF6) are implicated in glucose and energy metabolism $(135,142-144)$. Therefore, it is highly likely that UPR might play an important role in starvation induced adaptations in homeostasis including those in skeletal muscle. This study provides the first evidence that UPR and ER-stress are activated in skeletal muscle in response to food deprivation. Further, data from this study show that depletion of TRAF6 significantly inhibits upregulation of all markers of UPR in response to starvation in skeletal muscle. Together, this leads to a conclusion that UPR and ER-stress may play an important role in starvation-induced muscle atrophy and further that TRAF6 is of critical importance in activation of UPR in the conditions of food-deprivation.

In the same study, I showed that Fn14 (a TWEAK receptor) is highly induced in its expression in response to starvation, which is inhibited by TRAF6 depletion. However, our data from this study also show that inhibition of TWEAK-Fn14 system did not affect any of the major proteolytic systems and therefore, did not ameliorate starvation-induced atrophy.

In Chapter four, I have investigated the role of TRAF6 in skeletal muscle remodeling. TRAF6 is extensively studied in context of immune system. In an injuredmuscle microenvironment, cells of immune system have a significant intervention in processes of necrotic tissue removal and restoration of muscle architecture. Our previous data has already established a major regulatory role of TRAF6 in intracellular catabolic mechanisms in skeletal muscle. Therefore, TRAF6 may also influence skeletal muscle injury and consequential regeneration. The data from this study show that TRAF6 
expression is induced in response to injury. Data also show that TRAF6 regulates expression of several pro- and anti-inflammatory cytokines and some important chemokines. Similarly, TRAF6 induces expression of NF-אB in injured muscle. Through augmentation of inflammatory cytokines, chemokines and NF-kB, TRAF6 prolongs the inflammatory phase in a regenerating muscle. Our data reveal that TRAF6 ablation significantly inhibits the expression of pro-inflammatory cytokines, chemokines and transcription factors in regenerating muscle and thus promotes regeneration. Depletion of TRAF6 also reduces fibrosis in skeletal muscle. But the most important findings of this study are that TRAF6 mediates activation and proliferation of satellite cells and a shift in macrophage phenotype from M1 (proinflammatory) to M2c (pro-myogenic). Using two genetic mouse models of TRAF6 knock-out, one in developing myoblasts and the other in differentiated myotube, I showed that depletion of TRAF6 promotes activation and proliferation of muscle precursor cells (MPCs) which assists in accelerated regeneration. Further, this study also reveals that ablation of TRAF6 causes a shift in macrophage phenotype activation and thus contributing to an improved regeneration of skeletal muscle.

\subsection{Contributions of this Dissertation}

This dissertation has made significant contributions to scholarship in skeletal muscle metabolism. It has systematically identified, dissected and characterized the role of TRAF6 which is an important upstream signaling molecule in receptor-mediated activation of intracellular molecular mechanisms. 
Through a methodological and thorough review of literature on skeletal muscle catabolic conditions and response mechanism induced thereby, this dissertation has provided new insights into convergent role of upstream signaling molecules in skeletal muscle remodeling.

In addition, through a comprehensive investigation of interactions and regulatory roles of TRAF6 in intracellular mechanisms in skeletal muscle, this research work has revealed a novel signaling axis which modulates skeletal muscle homeostasis in stress conditions.

I anticipate this research will contribute to further research by assisting in development of new therapeutic strategies and interventions for the treatment of various catabolic conditions and disease states of skeletal muscle. Findings of this work may be of particular importance in elucidating the fine balance and interplay of factors critical for skeletal muscle remodeling.

Finally, this dissertation has provided useful insights and built a foundation for future research in regulation of proximal signaling events in skeletal muscle atrophy and regeneration.

\subsection{Limitations and Future Work}

As mentioned in sections $2.4,3.4$ and 4.4 , further research is needed to identify exactly how expression of TRAF6 is regulated in response to different catabolic signals. It also remains to be explored whether there are other mediators involved in execution of downstream effects of TRAF6. Also, more precise mechanism is yet to be known through which TRAF6 augments lysosomal degradation of muscle proteins and mitochondria. 
This research provides preliminary evidence that UPR and ER stress might be involved in starvation-induced skeletal muscle atrophy and that TRAF6 mediates activation of UPR/ER stress. This needs to be explored further how TRAF6 interacts with components of ER stress and/or UPR and what are the mechanisms through which this regulation occurs.

Further research is also needed to delineate the mechanisms which TRAF6 employs to influence the myeloid-myogenic interaction in injured or regenerating muscle. Although this work shows that TRAF6 may cause a bias in selection between macrophage phenotypes, it is yet to be understood whether this effect is exclusively through muscle intrinsic factors or yet to be characterized mediators are involved.

Finally, the rescue effects shown by TRAF6 deletion are not complete which leads to an assumption that there may be some overlap between functions of TRAF6 and other signaling molecules. Also, there is a possibility that there are other factors which exert similar effects as of TRAF6 but are controlled independently.

These are fascinating questions of great importance and answers to these will bring significant advancement in understanding of finely tuned functions of TRAF6 and will also improve the utility of the information provided by this dissertation in use of TRAF6 as a therapeutic target in muscle anomalies. 


\section{REFERENCES}

1. Jackman, R.W., and Kandarian, S.C. 2004. The molecular basis of skeletal muscle atrophy. Am J Physiol Cell Physiol 287:C834-843.

2. Kandarian, S.C., and Stevenson, E.J. 2002. Molecular events in skeletal muscle during disuse atrophy. Exerc Sport Sci Rev 30:111-116.

3. Lecker, S.H., Jagoe, R.T., Gilbert, A., Gomes, M., Baracos, V., Bailey, J., Price, S.R., Mitch, W.E., and Goldberg, A.L. 2004. Multiple types of skeletal muscle atrophy involve a common program of changes in gene expression. FASEB $J$ 18:39-51.

4. Porter, J.D., Khanna, S., Kaminski, H.J., Rao, J.S., Merriam, A.P., Richmonds, C.R., Leahy, P., Li, J., Guo, W., and Andrade, F.H. 2002. A chronic inflammatory response dominates the skeletal muscle molecular signature in dystrophindeficient mdx mice. Hum Mol Genet 11:263-272.

5. Zammit, P.S., Heslop, L., Hudon, V., Rosenblatt, J.D., Tajbakhsh, S., Buckingham, M.E., Beauchamp, J.R., and Partridge, T.A. 2002. Kinetics of myoblast proliferation show that resident satellite cells are competent to fully regenerate skeletal muscle fibers. Exp Cell Res 281:39-49.

6. Bartoli, M., and Richard, I. 2005. Calpains in muscle wasting. Int J Biochem Cell Biol 37:2115-2133.

7. Acharyya, S., Ladner, K.J., Nelsen, L.L., Damrauer, J., Reiser, P.J., Swoap, S., and Guttridge, D.C. 2004. Cancer cachexia is regulated by selective targeting of skeletal muscle gene products. JClin Invest 114:370-378.

8. Bhatnagar, S., Mittal, A., Gupta, S.K., and Kumar, A. 2011. TWEAK causes myotube atrophy through coordinated activation of ubiquitin-proteasome system, autophagy, and caspases. $J$ Cell Physiol:(in press). 
9. Bodine, S.C., Latres, E., Baumhueter, S., Lai, V.K., Nunez, L., Clarke, B.A., Poueymirou, W.T., Panaro, F.J., Na, E., Dharmarajan, K., et al. 2001. Identification of ubiquitin ligases required for skeletal muscle atrophy. Science 294:1704-1708.

10. Chen, Z.J. 2005. Ubiquitin signalling in the NF-kappaB pathway. Nat Cell Biol 7:758-765.

11. Lecker, S.H., Goldberg, A.L., and Mitch, W.E. 2006. Protein degradation by the ubiquitin-proteasome pathway in normal and disease states. $J$ Am Soc Nephrol 17:1807-1819.

12. Mittal, A., Bhatnagar, S., Kumar, A., Lach-Trifilieff, E., Wauters, S., Li, H., Makonchuk, D.Y., and Glass, D.J. 2010. The TWEAK-Fn14 system is a critical regulator of denervation-induced skeletal muscle atrophy in mice. $J$ Cell Biol 188:833-849.

13. Zhao, J., Brault, J.J., Schild, A., Cao, P., Sandri, M., Schiaffino, S., Lecker, S.H., and Goldberg, A.L. 2007. FoxO3 coordinately activates protein degradation by the autophagic/lysosomal and proteasomal pathways in atrophying muscle cells. Cell Metab 6:472-483.

14. Mammucari, C., Milan, G., Romanello, V., Masiero, E., Rudolf, R., Del Piccolo, P., Burden, S.J., Di Lisi, R., Sandri, C., Zhao, J., et al. 2007. FoxO3 controls autophagy in skeletal muscle in vivo. Cell Metab 6:458-471.

15. Masiero, E., Agatea, L., Mammucari, C., Blaauw, B., Loro, E., Komatsu, M., Metzger, D., Reggiani, C., Schiaffino, S., and Sandri, M. 2009. Autophagy is required to maintain muscle mass. Cell Metab 10:507-515.

16. Ogata, T., Oishi, Y., Higuchi, M., and Muraoka, I. 2010. Fasting-related autophagic response in slow- and fast-twitch skeletal muscle. Biochem Biophys Res Commun 394:136-140.

17. Romanello, V., Guadagnin, E., Gomes, L., Roder, I., Sandri, C., Petersen, Y., Milan, G., Masiero, E., Del Piccolo, P., Foretz, M., et al. 2010. Mitochondrial fission and remodelling contributes to muscle atrophy. EMBOJ 29:1774-1785.

18. Sandri, M. 2010. Autophagy in skeletal muscle. FEBS Lett 584:1411-1416. 
19. Jackson, P.K., Eldridge, A.G., Freed, E., Furstenthal, L., Hsu, J.Y., Kaiser, B.K., and Reimann, J.D. 2000. The lore of the RINGs: substrate recognition and catalysis by ubiquitin ligases. Trends Cell Biol 10:429-439.

20. Cox, J.S., Shamu, C.E., and Walter, P. 1993. Transcriptional induction of genes encoding endoplasmic reticulum resident proteins requires a transmembrane protein kinase. Cell 73:1197-1206.

21. Cong, H., Sun, L., Liu, C., and Tien, P. 2011. Inhibition of atrogin-1/MAFbx expression by adenovirus-delivered small hairpin RNAs attenuates muscle atrophy in fasting mice. Hum Gene Ther 22:313-324.

22. Dehoux, M., Van Beneden, R., Pasko, N., Lause, P., Verniers, J., Underwood, L., Ketelslegers, J.M., and Thissen, J.P. 2004. Role of the insulin-like growth factor I decline in the induction of atrogin-1/MAFbx during fasting and diabetes. Endocrinology 145:4806-4812.

23. Ebert, S.M., Monteys, A.M., Fox, D.K., Bongers, K.S., Shields, B.E., Malmberg, S.E., Davidson, B.L., Suneja, M., and Adams, C.M. 2010. The transcription factor ATF4 promotes skeletal myofiber atrophy during fasting. Mol Endocrinol 24:790799.

24. Doyle, A., Zhang, G., Abdel Fattah, E.A., Eissa, N.T., and Li, Y.P. 2011. Tolllike receptor 4 mediates lipopolysaccharide-induced muscle catabolism via coordinate activation of ubiquitin-proteasome and autophagy-lysosome pathways. FASEB J 25:99-110.

25. Foletta, V.C., White, L.J., Larsen, A.E., Leger, B., and Russell, A.P. 2011. The role and regulation of MAFbx/atrogin-1 and MuRF1 in skeletal muscle atrophy. Pflugers Arch 461:325-335.

26. Glass, D.J. 2005. Skeletal muscle hypertrophy and atrophy signaling pathways. Int J Biochem Cell Biol 37:1974-1984.

27. Gomes, M.D., Lecker, S.H., Jagoe, R.T., Navon, A., and Goldberg, A.L. 2001. Atrogin-1, a muscle-specific F-box protein highly expressed during muscle atrophy. Proc Natl Acad Sci US A 98:14440-14445.

28. Gonzalez-Cadavid, N.F., Taylor, W.E., Yarasheski, K., Sinha-Hikim, I., Ma, K., Ezzat, S., Shen, R., Lalani, R., Asa, S., Mamita, M., et al. 1998. Organization of 
the human myostatin gene and expression in healthy men and HIV-infected men with muscle wasting. Proc Natl Acad Sci US A 95:14938-14943.

29. Harding, H.P., Zhang, Y., and Ron, D. 1999. Protein translation and folding are coupled by an endoplasmic-reticulum-resident kinase. Nature 397:271-274.

30. Lamothe, B., Webster, W.K., Gopinathan, A., Besse, A., Campos, A.D., and Darnay, B.G. 2007. TRAF6 ubiquitin ligase is essential for RANKL signaling and osteoclast differentiation. Biochem Biophys Res Commun 359:1044-1049.

31. Hishiya, A., Iemura, S., Natsume, T., Takayama, S., Ikeda, K., and Watanabe, K. 2006. A novel ubiquitin-binding protein ZNF216 functioning in muscle atrophy. EMBO J 25:554-564.

32. Haze, K., Yoshida, H., Yanagi, H., Yura, T., and Mori, K. 1999. Mammalian transcription factor ATF6 is synthesized as a transmembrane protein and activated by proteolysis in response to endoplasmic reticulum stress. $\mathrm{Mol}$ Biol Cell 10:3787-3799.

33. Aguirre, V., Uchida, T., Yenush, L., Davis, R., and White, M.F. 2000. The c-Jun $\mathrm{NH}(2)$-terminal kinase promotes insulin resistance during association with insulin receptor substrate-1 and phosphorylation of Ser(307). J Biol Chem 275:90479054.

34. Kolodziejczyk, S.M., Walsh, G.S., Balazsi, K., Seale, P., Sandoz, J., Hierlihy, A.M., Rudnicki, M.A., Chamberlain, J.S., Miller, F.D., and Megeney, L.A. 2001. Activation of JNK1 contributes to dystrophic muscle pathogenesis. Curr Biol $11: 1278-1282$.

35. Liu, G., and Rondinone, C.M. 2005. JNK: bridging the insulin signaling and inflammatory pathway. Curr Opin Investig Drugs 6:979-987.

36. Supinski, G.S., Ji, X., and Callahan, L.A. 2009. The JNK MAP kinase pathway contributes to the development of endotoxin-induced diaphragm caspase activation. Am J Physiol Regul Integr Comp Physiol 297:R825-834.

37. Krawiec, B.J., Nystrom, G.J., Frost, R.A., Jefferson, L.S., and Lang, C.H. 2007. AMP-activated protein kinase agonists increase mRNA content of the musclespecific ubiquitin ligases MAFbx and MuRF1 in C2C12 cells. Am J Physiol Endocrinol Metab 292:E1555-1567. 
38. Meley, D., Bauvy, C., Houben-Weerts, J.H., Dubbelhuis, P.F., Helmond, M.T., Codogno, P., and Meijer, A.J. 2006. AMP-activated protein kinase and the regulation of autophagic proteolysis. $J$ Biol Chem 281:34870-34879.

39. Lamothe, B., Besse, A., Campos, A.D., Webster, W.K., Wu, H., and Darnay, B.G. 2007. Site-specific Lys-63-linked tumor necrosis factor receptor-associated factor 6 auto-ubiquitination is a critical determinant of I kappa B kinase activation. $J$ Biol Chem 282:4102-4112.

40. Lamothe, B., Campos, A.D., Webster, W.K., Gopinathan, A., Hur, L., and Darnay, B.G. 2008. The RING domain and first zinc finger of TRAF6 coordinate signaling by interleukin-1, lipopolysaccharide, and RANKL. J Biol Chem 283:24871-24880.

41. Sandri, M., Sandri, C., Gilbert, A., Skurk, C., Calabria, E., Picard, A., Walsh, K., Schiaffino, S., Lecker, S.H., and Goldberg, A.L. 2004. Foxo transcription factors induce the atrophy-related ubiquitin ligase atrogin-1 and cause skeletal muscle atrophy. Cell 117:399-412.

42. Stitt, T.N., Drujan, D., Clarke, B.A., Panaro, F., Timofeyva, Y., Kline, W.O., Gonzalez, M., Yancopoulos, G.D., and Glass, D.J. 2004. The IGF-1/PI3K/Akt pathway prevents expression of muscle atrophy-induced ubiquitin ligases by inhibiting FOXO transcription factors. Mol Cell 14:395-403.

43. Adhikari, A., Xu, M., and Chen, Z.J. 2007. Ubiquitin-mediated activation of TAK1 and IKK. Oncogene 26:3214-3226.

44. Dogra, C., Changotra, H., Wergedal, J.E., and Kumar, A. 2006. Regulation of phosphatidylinositol 3-kinase (PI3K)/Akt and nuclear factor-kappa B signaling pathways in dystrophin-deficient skeletal muscle in response to mechanical stretch. J Cell Physiol 208:575-585.

45. Gao, Z., Hwang, D., Bataille, F., Lefevre, M., York, D., Quon, M.J., and Ye, J. 2002. Serine phosphorylation of insulin receptor substrate 1 by inhibitor kappa $B$ kinase complex. J Biol Chem 277:48115-48121.

46. Cai, D., Frantz, J.D., Tawa, N.E., Jr., Melendez, P.A., Oh, B.C., Lidov, H.G., Hasselgren, P.O., Frontera, W.R., Lee, J., Glass, D.J., et al. 2004. IKKbeta/NFkappaB activation causes severe muscle wasting in mice. Cell 119:285-298. 
47. Li, H., Malhotra, S., and Kumar, A. 2008. Nuclear factor-kappa B signaling in skeletal muscle atrophy. $J$ Mol Med 86:1113-1126.

48. Nakamura, K., Kimple, A.J., Siderovski, D.P., and Johnson, G.L. 2010. PB1 domain interaction of p62/sequestosome 1 and MEKK3 regulates NF-kappaB activation. $J$ Biol Chem 285:2077-2089.

49. Ottnad, E., Parthasarathy, S., Sambrano, G.R., Ramprasad, M.P., Quehenberger, O., Kondratenko, N., Green, S., and Steinberg, D. 1995. A macrophage receptor for oxidized low density lipoprotein distinct from the receptor for acetyl low density lipoprotein: partial purification and role in recognition of oxidatively damaged cells. Proc Natl Acad Sci U S A 92:1391-1395.

50. Yahiaoui, L., Gvozdic, D., Danialou, G., Mack, M., and Petrof, B.J. 2008. CC family chemokines directly regulate myoblast responses to skeletal muscle injury. J Physiol 586:3991-4004.

51. Zador, E., Mendler, L., Takacs, V., de Bleecker, J., and Wuytack, F. 2001. Regenerating soleus and extensor digitorum longus muscles of the rat show elevated levels of TNF-alpha and its receptors, TNFR-60 and TNFR-80. Muscle Nerve 24:1058-1067.

52. Aktas, O., Prozorovski, T., and Zipp, F. 2006. Death ligands and autoimmune demyelination. Neuroscientist 12:305-316.

53. Mittal, A., Bhatnagar, S., Kumar, A., Lach-Trifilieff, E., Wauters, S., Li, H., Makonchuk, D.Y., Glass, D.J., and Kumar, A. 2010. The TWEAK-Fn14 system is a critical regulator of denervation-induced skeletal muscle atrophy in mice. $J$ Cell Biol 188:833-849.

54. Eash, J., Olsen, A., Breur, G., Gerrard, D., and Hannon, K. 2007. FGFR1 inhibits skeletal muscle atrophy associated with hindlimb suspension. BMC Musculoskelet Disord 8:32.

55. Novak, M.L., Billich, W., Smith, S.M., Sukhija, K.B., McLoughlin, T.J., Hornberger, T.A., and Koh, T.J. 2009. COX-2 inhibitor reduces skeletal muscle hypertrophy in mice. Am J Physiol Regul Integr Comp Physiol 296:R1132-1139.

56. Lee, N.K., and Lee, S.Y. 2002. Modulation of life and death by the tumor necrosis factor receptor-associated factors (TRAFs). J Biochem Mol Biol 35:61-66. 
57. Thakur, N., Sorrentino, A., Heldin, C.H., and Landstrom, M. 2009. TGF-beta uses the E3-ligase TRAF6 to turn on the kinase TAK1 to kill prostate cancer cells. Future Oncol 5:1-3.

58. Arch, R.H., Gedrich, R.W., and Thompson, C.B. 1998. Tumor necrosis factor receptor-associated factors (TRAFs)--a family of adapter proteins that regulates life and death. Genes Dev 12:2821-2830.

59. Chung, J.Y., Park, Y.C., Ye, H., and Wu, H. 2002. All TRAFs are not created equal: common and distinct molecular mechanisms of TRAF-mediated signal transduction. J Cell Sci 115:679-688.

60. Xu, L.G., Li, L.Y., and Shu, H.B. 2004. TRAF7 potentiates MEKK3-induced AP1 and CHOP activation and induces apoptosis. J Biol Chem 279:17278-17282.

61. Kaufman, D.R., and Choi, Y. 1999. Signaling by tumor necrosis factor receptors: pathways, paradigms and targets for therapeutic modulation. Int Rev Immunol $18: 405-427$.

62. Hsu, H., Shu, H.B., Pan, M.G., and Goeddel, D.V. 1996. TRADD-TRAF2 and TRADD-FADD interactions define two distinct TNF receptor 1 signal transduction pathways. Cell 84:299-308.

63. Sun, L., and Chen, Z.J. 2004. The novel functions of ubiquitination in signaling. Curr Opin Cell Biol 16:119-126.

64. Pickart, C.M. 2001. Mechanisms underlying ubiquitination. Annu Rev Biochem 70:503-533.

65. Mukhopadhyay, D., and Riezman, H. 2007. Proteasome-independent functions of ubiquitin in endocytosis and signaling. Science 315:201-205.

66. Wang, C., Deng, L., Hong, M., Akkaraju, G.R., Inoue, J., and Chen, Z.J. 2001. TAK1 is a ubiquitin-dependent kinase of MKK and IKK. Nature 412:346-351.

67. Ye, H., Arron, J.R., Lamothe, B., Cirilli, M., Kobayashi, T., Shevde, N.K., Segal, D., Dzivenu, O.K., Vologodskaia, M., Yim, M., et al. 2002. Distinct molecular mechanism for initiating TRAF6 signalling. Nature 418:443-447. 
68. Lomaga, M.A., Yeh, W.C., Sarosi, I., Duncan, G.S., Furlonger, C., Ho, A., Morony, S., Capparelli, C., Van, G., Kaufman, S., et al. 1999. TRAF6 deficiency results in osteopetrosis and defective interleukin-1, CD40, and LPS signaling. Genes Dev 13:1015-1024.

69. Kim, N., Kadono, Y., Takami, M., Lee, J., Lee, S.H., Okada, F., Kim, J.H., Kobayashi, T., Odgren, P.R., Nakano, H., et al. 2005. Osteoclast differentiation independent of the TRANCE-RANK-TRAF6 axis. J Exp Med 202:589-595.

70. Jundi, M., Nadiri, A., Al-Zoobi, L., Hassan, G.S., and Mourad, W. 2011. CD40mediated cell death requires TRAF6 recruitment. Immunobiology.

71. Lutgens, E., Lievens, D., Beckers, L., Wijnands, E., Soehnlein, O., Zernecke, A., Seijkens, T., Engel, D., Cleutjens, J., Keller, A.M., et al. 2010. Deficient CD40TRAF6 signaling in leukocytes prevents atherosclerosis by skewing the immune response toward an antiinflammatory profile. $J$ Exp Med 207:391-404.

72. Pollet, I., Opina, C.J., Zimmerman, C., Leong, K.G., Wong, F., and Karsan, A. 2003. Bacterial lipopolysaccharide directly induces angiogenesis through TRAF6mediated activation of NF-kappaB and c-Jun N-terminal kinase. Blood 102:17401742.

73. Zhong, J., and Kyriakis, J.M. 2004. Germinal center kinase is required for optimal Jun N-terminal kinase activation by Toll-like receptor agonists and is regulated by the ubiquitin proteasome system and agonist-induced, TRAF6-dependent stabilization. Mol Cell Biol 24:9165-9175.

74. Yamaguchi, N., Kukita, T., Li, Y.J., Kamio, N., Fukumoto, S., Nonaka, K., Ninomiya, Y., Hanazawa, S., and Yamashita, Y. 2008. Adiponectin inhibits induction of TNF-alpha/RANKL-stimulated NFATc1 via the AMPK signaling. FEBS Lett 582:451-456.

75. Yamashita, M., Fatyol, K., Jin, C., Wang, X., Liu, Z., and Zhang, Y.E. 2008. TRAF6 mediates Smad-independent activation of JNK and p38 by TGF-beta. $\mathrm{Mol}$ Cell 31:918-924.

76. Yang, W.L., Wang, J., Chan, C.H., Lee, S.W., Campos, A.D., Lamothe, B., Hur, L., Grabiner, B.C., Lin, X., Darnay, B.G., et al. 2009. The E3 ligase TRAF6 regulates Akt ubiquitination and activation. Science 325:1134-1138. 
77. Moscat, J., Diaz-Meco, M.T., and Wooten, M.W. 2007. Signal integration and diversification through the p62 scaffold protein. Trends Biochem Sci 32:95-100.

78. Winkles, J.A. 2008. The TWEAK-Fn14 cytokine-receptor axis: discovery, biology and therapeutic targeting. Nat Rev Drug Discov 7:411-425.

79. Martinon, F., Chen, X., Lee, A.H., and Glimcher, L.H. 2010. TLR activation of the transcription factor XBP1 regulates innate immune responses in macrophages. Nat Immunol 11:411-418.

80. Mourkioti, F., and Rosenthal, N. 2008. NF-kappaB signaling in skeletal muscle: prospects for intervention in muscle diseases. J Mol Med (Berl) 86:747-759.

81. Glass, D.J. 2003. Molecular mechanisms modulating muscle mass. Trends Mol Med 9:344-350.

82. Cao, P.R., Kim, H.J., and Lecker, S.H. 2005. Ubiquitin-protein ligases in muscle wasting. Int J Biochem Cell Biol 37:2088-2097.

83. Solomon, V., and Goldberg, A.L. 1996. Importance of the ATP-ubiquitinproteasome pathway in the degradation of soluble and myofibrillar proteins in rabbit muscle extracts. $J$ Biol Chem 271:26690-26697.

84. Glass, D.J. 2010. Signaling pathways perturbing muscle mass. Curr Opin Clin Nutr Metab Care 13:225-229.

85. Kumar, A., Murphy, R., Robinson, P., Wei, L., and Boriek, A.M. 2004. Cyclic mechanical strain inhibits skeletal myogenesis through activation of focal adhesion kinase, Rac-1 GTPase, and NF-kappaB transcription factor. FASEB J 18:1524-1535.

86. Mourkioti, F., Kratsios, P., Luedde, T., Song, Y.H., Delafontaine, P., Adami, R., Parente, V., Bottinelli, R., Pasparakis, M., and Rosenthal, N. 2006. Targeted ablation of IKK2 improves skeletal muscle strength, maintains mass, and promotes regeneration. $J$ Clin Invest 116:2945-2954.

87. Li, Y.P., Chen, Y., John, J., Moylan, J., Jin, B., Mann, D.L., and Reid, M.B. 2005. TNF-alpha acts via p38 MAPK to stimulate expression of the ubiquitin ligase atrogin1/MAFbx in skeletal muscle. FASEB J 19:362-370. 
88. Criollo, A., Senovilla, L., Authier, H., Maiuri, M.C., Morselli, E., Vitale, I., Kepp, O., Tasdemir, E., Galluzzi, L., Shen, S., et al. 2010. The IKK complex contributes to the induction of autophagy. EMBOJ 29:619-631.

89. Criollo, A., Senovilla, L., Authier, H., Maiuri, M.C., Morselli, E., Vitale, I., Kepp, O., Tasdemir, E., Galluzzi, L., Shen, S., et al. 2010. IKK connects autophagy to major stress pathways. Autophagy 6:189-191.

90. Aggarwal, B.B. 2003. Signalling pathways of the TNF superfamily: a doubleedged sword. Nat Rev Immunol 3:745-756.

91. Seibenhener, M.L., Babu, J.R., Geetha, T., Wong, H.C., Krishna, N.R., and Wooten, M.W. 2004. Sequestosome $1 / \mathrm{p} 62$ is a polyubiquitin chain binding protein involved in ubiquitin proteasome degradation. Mol Cell Biol 24:80558068.

92. Wooten, M.W., Geetha, T., Seibenhener, M.L., Babu, J.R., Diaz-Meco, M.T., and Moscat, J. 2005. The p62 scaffold regulates nerve growth factor-induced NFkappaB activation by influencing TRAF6 polyubiquitination. $J$ Biol Chem $280: 35625-35629$.

93. Shi, C.S., and Kehrl, J.H. 2010. TRAF6 and A20 regulate lysine 63-linked ubiquitination of Beclin-1 to control TLR4-induced autophagy. Sci Signal 3:ra42.

94. Kobayashi, T., Walsh, P.T., Walsh, M.C., Speirs, K.M., Chiffoleau, E., King, C.G., Hancock, W.W., Caamano, J.H., Hunter, C.A., Scott, P., et al. 2003. TRAF6 is a critical factor for dendritic cell maturation and development. Immunity 19:353-363.

95. Naito, A., Azuma, S., Tanaka, S., Miyazaki, T., Takaki, S., Takatsu, K., Nakao, K., Nakamura, K., Katsuki, M., Yamamoto, T., et al. 1999. Severe osteopetrosis, defective interleukin-1 signalling and lymph node organogenesis in TRAF6deficient mice. Genes Cells 4:353-362.

96. Bruning, J.C., Michael, M.D., Winnay, J.N., Hayashi, T., Horsch, D., Accili, D., Goodyear, L.J., and Kahn, C.R. 1998. A muscle-specific insulin receptor knockout exhibits features of the metabolic syndrome of NIDDM without altering glucose tolerance. Mol Cell 2:559-569. 
97. Baba, S.P., Barski, O.A., Ahmed, Y., O'Toole, T.E., Conklin, D.J., Bhatnagar, A., and Srivastava, S. 2009. Reductive metabolism of AGE precursors: a metabolic route for preventing $\mathrm{AGE}$ accumulation in cardiovascular tissue. Diabetes $58: 2486-2497$.

98. Dogra, C., Changotra, H., Mohan, S., and Kumar, A. 2006. Tumor necrosis factor-like weak inducer of apoptosis inhibits skeletal myogenesis through sustained activation of nuclear factor-kappaB and degradation of MyoD protein. $J$ Biol Chem 281:10327-10336.

99. Dogra, C., Changotra, H., Wedhas, N., Qin, X., Wergedal, J.E., and Kumar, A. 2007. TNF-related weak inducer of apoptosis (TWEAK) is a potent skeletal muscle-wasting cytokine. FASEB J 21:1857-1869.

100. Kumar, A., and Boriek, A.M. 2003. Mechanical stress activates the nuclear factorkappaB pathway in skeletal muscle fibers: a possible role in Duchenne muscular dystrophy. FASEB J 17:386-396.

101. Li, H., Mittal, A., Makonchuk, D.Y., Bhatnagar, S., and Kumar, A. 2009. Matrix Metalloproteinase-9 Inhibition Ameliorates Pathogenesis and Improves Skeletal Muscle Regeneration in Muscular Dystrophy. Hum Mol Genet 18:2584-2598.

102. Acharyya, S., and Guttridge, D.C. 2007. Cancer cachexia signaling pathways continue to emerge yet much still points to the proteasome. Clin Cancer Res 13:1356-1361.

103. Chung, J.Y., Lu, M., Yin, Q., Lin, S.C., and Wu, H. 2007. Molecular basis for the unique specificity of TRAF6. Adv Exp Med Biol 597:122-130.

104. Zapata, J.M., Lefebvre, S., and Reed, J.C. 2007. Targeting TRAfs for therapeutic intervention. Adv Exp Med Biol 597:188-201.

105. Clarke, B.A., Drujan, D., Willis, M.S., Murphy, L.O., Corpina, R.A., Burova, E., Rakhilin, S.V., Stitt, T.N., Patterson, C., Latres, E., et al. 2007. The E3 Ligase MuRF1 degrades myosin heavy chain protein in dexamethasone-treated skeletal muscle. Cell Metab 6:376-385.

106. Kedar, V., McDonough, H., Arya, R., Li, H.H., Rockman, H.A., and Patterson, C. 2004. Muscle-specific RING finger 1 is a bona fide ubiquitin ligase that degrades cardiac troponin I. Proc Natl Acad Sci US A 101:18135-18140. 
107. Tintignac, L.A., Lagirand, J., Batonnet, S., Sirri, V., Leibovitch, M.P., and Leibovitch, S.A. 2005. Degradation of MyoD mediated by the SCF (MAFbx) ubiquitin ligase. $J$ Biol Chem 280:2847-2856.

108. Cohen, S., Brault, J.J., Gygi, S.P., Glass, D.J., Valenzuela, D.M., Gartner, C., Latres, E., and Goldberg, A.L. 2009. During muscle atrophy, thick, but not thin, filament components are degraded by MuRF1-dependent ubiquitylation. $J$ Cell Biol 185:1083-1095.

109. Chen, Y.W., Nagaraju, K., Bakay, M., McIntyre, O., Rawat, R., Shi, R., and Hoffman, E.P. 2005. Early onset of inflammation and later involvement of TGFbeta in Duchenne muscular dystrophy. Neurology 65:826-834.

110. Shim, J.H., Xiao, C., Paschal, A.E., Bailey, S.T., Rao, P., Hayden, M.S., Lee, K.Y., Bussey, C., Steckel, M., Tanaka, N., et al. 2005. TAK1, but not TAB1 or $\mathrm{TAB} 2$, plays an essential role in multiple signaling pathways in vivo. Genes Dev 19:2668-2681.

111. Gamboa, J.L., Garcia-Cazarin, M.L., and Andrade, F.H. 2011. Chronic hypoxia increases insulin-stimulated glucose uptake in mouse soleus muscle. Am J Physiol Regul Integr Comp Physiol 300:R85-91.

112. Argiles, J.M., Busquets, S., and Lopez-Soriano, F.J. 2005. The pivotal role of cytokines in muscle wasting during cancer. Int J Biochem Cell Biol 37:20362046.

113. Spate, U., and Schulze, P.C. 2004. Proinflammatory cytokines and skeletal muscle. Curr Opin Clin Nutr Metab Care 7:265-269.

114. Wing, S.S., and Goldberg, A.L. 1993. Glucocorticoids activate the ATPubiquitin-dependent proteolytic system in skeletal muscle during fasting. $\mathrm{Am} \mathrm{J}$ Physiol 264:E668-676.

115. Sacheck, J.M., Ohtsuka, A., McLary, S.C., and Goldberg, A.L. 2004. IGF-I stimulates muscle growth by suppressing protein breakdown and expression of atrophy-related ubiquitin ligases, atrogin-1 and MuRF1. Am J Physiol Endocrinol Metab 287:E591-601.

116. Silke, J., and Brink, R. 2010. Regulation of TNFRSF and innate immune signalling complexes by TRAFs and cIAPs. Cell Death Differ 17:35-45. 
117. Paul, P.K., Gupta, S.K., Bhatnagar, S., Panguluri, S.K., Darnay, B.G., Choi, Y., and Kumar, A. 2010. Targeted ablation of TRAF6 inhibits skeletal muscle wasting in mice. J Cell Biol 191:1395-1411.

118. Kozutsumi, Y., Segal, M., Normington, K., Gething, M.J., and Sambrook, J. 1988. The presence of malfolded proteins in the endoplasmic reticulum signals the induction of glucose-regulated proteins. Nature 332:462-464.

119. Kilberg, M.S., Shan, J., and Su, N. 2009. ATF4-dependent transcription mediates signaling of amino acid limitation. Trends Endocrinol Metab 20:436-443.

120. Wu, J., Ruas, J.L., Estall, J.L., Rasbach, K.A., Choi, J.H., Ye, L., Bostrom, P., Tyra, H.M., Crawford, R.W., Campbell, K.P., et al. 2011. The unfolded protein response mediates adaptation to exercise in skeletal muscle through a PGC1alpha/ATF6alpha complex. Cell Metab 13:160-169.

121. Schertzer, J.D., Plant, D.R., and Lynch, G.S. 2006. Optimizing plasmid-based gene transfer for investigating skeletal muscle structure and function. Mol Ther 13:795-803.

122. Rock, K.L., Gramm, C., Rothstein, L., Clark, K., Stein, R., Dick, L., Hwang, D., and Goldberg, A.L. 1994. Inhibitors of the proteasome block the degradation of most cell proteins and the generation of peptides presented on MHC class I molecules. Cell 78:761-771.

123. Schwartz, A.L., and Ciechanover, A. 1999. The ubiquitin-proteasome pathway and pathogenesis of human diseases. Annu Rev Med 50:57-74.

124. Ciechanover, A. 1998. The ubiquitin-proteasome pathway: on protein death and cell life. EMBO J 17:7151-7160.

125. Mizushima, N., Yamamoto, A., Matsui, M., Yoshimori, T., and Ohsumi, Y. 2004. In vivo analysis of autophagy in response to nutrient starvation using transgenic mice expressing a fluorescent autophagosome marker. Mol Biol Cell 15:11011111.

126. Levine, B., and Kroemer, G. 2008. Autophagy in the pathogenesis of disease. Cell 132:27-42. 
127. Bjorkoy, G., Lamark, T., Brech, A., Outzen, H., Perander, M., Overvatn, A., Stenmark, H., and Johansen, T. 2005. p62/SQSTM1 forms protein aggregates degraded by autophagy and has a protective effect on huntingtin-induced cell death. J Cell Biol 171:603-614.

128. Tran, H., Brunet, A., Griffith, E.C., and Greenberg, M.E. 2003. The many forks in FOXO's road. Sci STKE 2003:RE5.

129. Yoshida, H., Matsui, T., Yamamoto, A., Okada, T., and Mori, K. 2001. XBP1 mRNA is induced by ATF6 and spliced by IRE1 in response to ER stress to produce a highly active transcription factor. Cell 107:881-891.

130. Walsh, M.C., Kim, G.K., Maurizio, P.L., Molnar, E.E., and Choi, Y. 2008. TRAF6 autoubiquitination-independent activation of the NFkappaB and MAPK pathways in response to IL-1 and RANKL. PLoS One 3:e4064.

131. Rana, Z.A., Ekmark, M., and Gundersen, K. 2004. Coexpression after electroporation of plasmid mixtures into muscle in vivo. Acta Physiologica Scandinavica 181:233-238.

132. Bechet, D., Tassa, A., Taillandier, D., Combaret, L., and Attaix, D. 2005. Lysosomal proteolysis in skeletal muscle. Int J Biochem Cell Biol 37:2098-2114.

133. Ron, D., and Walter, P. 2007. Signal integration in the endoplasmic reticulum unfolded protein response. Nat Rev Mol Cell Biol 8:519-529.

134. Back, S.H., Scheuner, D., Han, J., Song, B., Ribick, M., Wang, J., Gildersleeve, R.D., Pennathur, S., and Kaufman, R.J. 2009. Translation attenuation through eIF2alpha phosphorylation prevents oxidative stress and maintains the differentiated state in beta cells. Cell Metab 10:13-26.

135. Oyadomari, S., Harding, H.P., Zhang, Y., Oyadomari, M., and Ron, D. 2008. Dephosphorylation of translation initiation factor 2alpha enhances glucose tolerance and attenuates hepatosteatosis in mice. Cell Metab 7:520-532.

136. Gregor, M.G., and Hotamisligil, G.S. 2007. Adipocyte stress: The endoplasmic reticulum and metabolic disease. $J$ Lipid Res. 
137. Rutkowski, D.T., Wu, J., Back, S.H., Callaghan, M.U., Ferris, S.P., Iqbal, J., Clark, R., Miao, H., Hassler, J.R., Fornek, J., et al. 2008. UPR pathways combine to prevent hepatic steatosis caused by ER stress-mediated suppression of transcriptional master regulators. Dev Cell 15:829-840.

138. Hu, P., Han, Z., Couvillon, A.D., Kaufman, R.J., and Exton, J.H. 2006. Autocrine tumor necrosis factor alpha links endoplasmic reticulum stress to the membrane death receptor pathway through IRE1alpha-mediated NF-kappaB activation and down-regulation of TRAF2 expression. Mol Cell Biol 26:3071-3084.

139. Yamazaki, H., Hiramatsu, N., Hayakawa, K., Tagawa, Y., Okamura, M., Ogata, R., Huang, T., Nakajima, S., Yao, J., Paton, A.W., et al. 2009. Activation of the Akt-NF-kappaB pathway by subtilase cytotoxin through the ATF6 branch of the unfolded protein response. $J$ Immunol 183:1480-1487.

140. Zhang, K., Shen, X., Wu, J., Sakaki, K., Saunders, T., Rutkowski, D.T., Back, S.H., and Kaufman, R.J. 2006. Endoplasmic reticulum stress activates cleavage of CREBH to induce a systemic inflammatory response. Cell 124:587-599.

141. Hunter, R.B., Mitchell-Felton, H., Essig, D.A., and Kandarian, S.C. 2001. Expression of endoplasmic reticulum stress proteins during skeletal muscle disuse atrophy. Am J Physiol Cell Physiol 281:C1285-1290.

142. Lee, A.H., Scapa, E.F., Cohen, D.E., and Glimcher, L.H. 2008. Regulation of hepatic lipogenesis by the transcription factor XBP1. Science 320:1492-1496.

143. Wang, Y., Vera, L., Fischer, W.H., and Montminy, M. 2009. The CREB coactivator CRTC2 links hepatic ER stress and fasting gluconeogenesis. Nature 460:534-537.

144. Acosta-Alvear, D., Zhou, Y., Blais, A., Tsikitis, M., Lents, N.H., Arias, C., Lennon, C.J., Kluger, Y., and Dynlacht, B.D. 2007. XBP1 controls diverse cell type- and condition-specific transcriptional regulatory networks. Mol Cell 27:5366.

145. Kim, I., Xu, W., and Reed, J.C. 2008. Cell death and endoplasmic reticulum stress: disease relevance and therapeutic opportunities. Nat Rev Drug Discov 7:1013-1030. 
146. Charge, S.B., and Rudnicki, M.A. 2004. Cellular and molecular regulation of muscle regeneration. Physiol Rev 84:209-238.

147. Dhawan, J., and Rando, T.A. 2005. Stem cells in postnatal myogenesis: molecular mechanisms of satellite cell quiescence, activation and replenishment. Trends Cell Biol 15:666-673.

148. Mauro, A. 1961. Satellite cell of skeletal muscle fibers. J Biophys Biochem Cytol 9:493-495.

149. Kuang, S., and Rudnicki, M.A. 2008. The emerging biology of satellite cells and their therapeutic potential. Trends Mol Med 14:82-91.

150. Tedesco, F.S., Dellavalle, A., Diaz-Manera, J., Messina, G., and Cossu, G. 2010. Repairing skeletal muscle: regenerative potential of skeletal muscle stem cells. $J$ Clin Invest 120:1 1-19.

151. Ten Broek, R.W., Grefte, S., and Von den Hoff, J.W. 2010. Regulatory factors and cell populations involved in skeletal muscle regeneration. $J$ Cell Physiol 224:7-16.

152. Tidball, J.G., and Villalta, S.A. 2010. Regulatory interactions between muscle and the immune system during muscle regeneration. Am J Physiol Regul Integr Comp Physiol 298:R1173-1187.

153. Bradley, J.R., and Pober, J.S. 2001. Tumor necrosis factor receptor-associated factors (TRAFs). Oncogene 20:6482-6491.

154. Bartoli, C., Civatte, M., Pellissier, J.F., and Figarella-Branger, D. 2001. CCR2A and CCR2B, the two isoforms of the monocyte chemoattractant protein-1 receptor are up-regulated and expressed by different cell subsets in idiopathic inflammatory myopathies. Acta Neuropathol 102:385-392.

155. Villalta, S.A., Nguyen, H.X., Deng, B., Gotoh, T., and Tidball, J.G. 2009. Shifts in macrophage phenotypes and macrophage competition for arginine metabolism affect the severity of muscle pathology in muscular dystrophy. Hum Mol Genet $18: 482-496$. 
156. Baeza-Raja, B., and Munoz-Canoves, P. 2004. p38 MAPK-induced nuclear factor-kappaB activity is required for skeletal muscle differentiation: role of interleukin-6. Mol Biol Cell 15:2013-2026.

157. Perdiguero, E., Ruiz-Bonilla, V., Gresh, L., Hui, L., Ballestar, E., Sousa-Victor, P., Baeza-Raja, B., Jardi, M., Bosch-Comas, A., Esteller, M., et al. 2007. Genetic analysis of p38 MAP kinases in myogenesis: fundamental role of p38alpha in abrogating myoblast proliferation. EMBO J 26:1245-1256.

158. Warren, G.L., O'Farrell, L., Summan, M., Hulderman, T., Mishra, D., Luster, M.I., Kuziel, W.A., and Simeonova, P.P. 2004. Role of CC chemokines in skeletal muscle functional restoration after injury. Am J Physiol Cell Physiol 286:C1031-1036.

159. Gillespie, M.A., Le Grand, F., Scime, A., Kuang, S., von Maltzahn, J., Seale, V., Cuenda, A., Ranish, J.A., and Rudnicki, M.A. 2009. p38-\{gamma\}-dependent gene silencing restricts entry into the myogenic differentiation program. $J$ Cell Biol 187:991-1005.

160. Reaich, D., Channon, S.M., Scrimgeour, C.M., Daley, S.E., Wilkinson, R., and Goodship, T.H. 1993. Correction of acidosis in humans with CRF decreases protein degradation and amino acid oxidation. Am J Physiol 265:E230-235.

161. Dogra, C., Hall, S.L., Wedhas, N., Linkhart, T.A., and Kumar, A. 2007. Fibroblast growth factor inducible 14 (Fn14) is required for the expression of myogenic regulatory factors and differentiation of myoblasts into myotubes. Evidence for TWEAK-independent functions of Fn14 during myogenesis. $J$ Biol Chem 282:15000-15010.

162. Kuang, S., Kuroda, K., Le Grand, F., and Rudnicki, M.A. 2007. Asymmetric selfrenewal and commitment of satellite stem cells in muscle. Cell 129:999-1010.

163. Apponi, L.H., Leung, S.W., Williams, K.R., Valentini, S.R., Corbett, A.H., and Pavlath, G.K. 2010. Loss of nuclear poly(A)-binding protein 1 causes defects in myogenesis and mRNA biogenesis. Hum Mol Genet 19:1058-1065.

164. Rando, T.A., and Blau, H.M. 1994. Primary mouse myoblast purification, characterization, and transplantation for cell-mediated gene therapy. J Cell Biol 125:1275-1287. 
165. Dogra, C., Changotra, H., Mohan, S., and Kumar, A. 2006. Tumor necrosis factor-like weak inducer of apoptosis inhibits skeletal myogenesis through sustained activation of nuclear factor-kappaB and degradation of MyoD protein. $J$ Biol Chem. 281:10327-10336.

166. Hawke, T.J., and Garry, D.J. 2001. Myogenic satellite cells: physiology to molecular biology. J Appl Physiol 91:534-551.

167. Yablonka-Reuveni, Z., and Rivera, A.J. 1994. Temporal expression of regulatory and structural muscle proteins during myogenesis of satellite cells on isolated adult rat fibers. Dev Biol 164:588-603.

168. Yan, Z., Choi, S., Liu, X., Zhang, M., Schageman, J.J., Lee, S.Y., Hart, R., Lin, L., Thurmond, F.A., and Williams, R.S. 2003. Highly coordinated gene regulation in mouse skeletal muscle regeneration. $J$ Biol Chem 278:8826-8836.

169. Gordon, S. 2003. Alternative activation of macrophages. Nat Rev Immunol 3:2335.

170. Sacheck, J.M., Hyatt, J.P., Raffaello, A., Jagoe, R.T., Roy, R.R., Edgerton, V.R., Lecker, S.H., and Goldberg, A.L. 2007. Rapid disuse and denervation atrophy involve transcriptional changes similar to those of muscle wasting during systemic diseases. FASEB J 21:140-155.

171. Wu, H., and Arron, J.R. 2003. TRAF6, a molecular bridge spanning adaptive immunity, innate immunity and osteoimmunology. Bioessays 25:1096-1105.

172. Matsuba, Y., Goto, K., Morioka, S., Naito, T., Akema, T., Hashimoto, N., Sugiura, T., Ohira, Y., Beppu, M., and Yoshioka, T. 2009. Gravitational unloading inhibits the regenerative potential of atrophied soleus muscle in mice. Acta Physiol (Oxf) 196:329-339.

173. Williams, J.L., Cartland, D., Hussain, A., and Egginton, S. 2006. A differential role for nitric oxide in two forms of physiological angiogenesis in mouse. $J$ Physiol 570:445-454.

174. Huh, M.S., Parker, M.H., Scime, A., Parks, R., and Rudnicki, M.A. 2004. Rb is required for progression through myogenic differentiation but not maintenance of terminal differentiation. J Cell Biol 166:865-876. 
175. Tallquist, M.D., Weismann, K.E., Hellstrom, M., and Soriano, P. 2000. Early myotome specification regulates PDGFA expression and axial skeleton development. Development 127:5059-5070.

176. Chen, S.E., Jin, B., and Li, Y.P. 2007. TNF-alpha regulates myogenesis and muscle regeneration by activating p38 MAPK. Am J Physiol Cell Physiol 292:C1660-1671.

177. Zoico, E., and Roubenoff, R. 2002. The role of cytokines in regulating protein metabolism and muscle function. Nutr Rev 60:39-51.

178. Allen, R.E., and Boxhorn, L.K. 1989. Regulation of skeletal muscle satellite cell proliferation and differentiation by transforming growth factor-beta, insulin-like growth factor I, and fibroblast growth factor. J Cell Physiol 138:311-315.

179. Guttridge, D.C., Albanese, C., Reuther, J.Y., Pestell, R.G., and Baldwin, A.S., Jr. 1999. NF-kappaB controls cell growth and differentiation through transcriptional regulation of cyclin D1. Mol Cell Biol 19:5785-5799.

180. Grounds, M.D. 1987. Phagocytosis of necrotic muscle in muscle isografts is influenced by the strain, age, and sex of host mice. $J$ Pathol 153:71-82.

181. Marino, J.S., Tausch, B.J., Dearth, C.L., Manacci, M.V., McLoughlin, T.J., Rakyta, S.J., Linsenmayer, M.P., and Pizza, F.X. 2008. Beta2-integrins contribute to skeletal muscle hypertrophy in mice. Am J Physiol Cell Physiol 295:C10261036.

182. Schwartz, L.M. 2008. Atrophy and programmed cell death of skeletal muscle. Cell Death Differ 15:1163-1169.

183. Irintchev, A., Zweyer, M., and Wernig, A. 1997. Impaired functional and structural recovery after muscle injury in dystrophic mdx mice. Neuromuscul Disord 7:117-125.

184. Smith, C.K., 2nd, Janney, M.J., and Allen, R.E. 1994. Temporal expression of myogenic regulatory genes during activation, proliferation, and differentiation of rat skeletal muscle satellite cells. J Cell Physiol 159:379-385. 


\section{APPENDICES}

\section{APPENDIX-1}

\begin{tabular}{|l|l|l|}
\hline Gene name & Forward primer (5'-3') & Reverse primer (5'-3') \\
\hline TRAF6 exon 7 & GGGAGCTGACTGCCAAAATG & GCGCTGAGCTGTCGGTAACT \\
\hline TRAF6 & GCAGTGAAAGATGACAGCGTGA & TCCCGTAAAGCCATCAAGCA \\
\hline IRES & CTAACGTTACTGGCCGAAGC & AGGAACTGCTTCCTTCACGA \\
\hline MHC4 & CGGCAATGAGTACGTCACCAAA & TCAAAGCCAGCGATGTCCAA \\
\hline Atrogin- 1 & GTCGCAGCCAAGAAGAGAAAGA & TGCTATCAGCTCCAACAGCCTT \\
\hline MuRF 1 & TAACTGCATCTCCATGCTGGTG & TGGCGTAGAGGGTGTCAAACTT \\
\hline$T N F-\alpha$ & GCATGATCCGCGACGTGGAA & AGATCCATGCCGTTGGCCAG \\
\hline Mac-1 1 & AGGGTTGTCCAGCCGATGATAT & CCCAGCTTCTTGACGTTGTTGA \\
\hline CD 8 & TTACTCTCCTGCCATCCTTCACGA & CCATTTGTGGTGGGAGAAACTGTG \\
\hline IL-1 $\beta$ & CTCCATGAGCTTTGTACAAGG & TGCTGATGTACCAGTTGGGG \\
\hline Tropomyosin A & ACATTGCTGAAGATGCTGACCG & TTCAAGCTCGGCACATTTGC \\
\hline Pax-7 & CAGTGTGCCATCTACCCATGCTTA & GGTGCTTGGTTCAAATTGAGCC \\
\hline LC3B & CTGGTGAATGGGCACAGCATG & CGTCCGCTGGTAACATCCCTT \\
\hline Beclin 1 & TGAAATCAATGCTGCCTGGG & CCAGAACAGTATAACGGCAACTCC \\
\hline Gabarapl1 & CGGTCATCGTGGAGAAGGCT & CCAGAACAGTATAACGGCAACTCC \\
\hline$\beta$-actin & CAGGCATTGCTGACAGGATG & TGCTGATCCACATCTGCTGG \\
\hline GAPDH & ATGACAATGAATACGGCTACAGCAA & GCAGCGAACTTTATTGATGGTATT \\
\hline
\end{tabular}




\section{APPENDIX-2}

\section{LIST OF ABBREVIATIONS}
ALS. autophagy-lysosome system AMPK AMP activated protein kinase ATF...........................activating transcription factor CCL...........................chemokine ligand cDNA......................... complimentary deoxyribonucleic acid CMA............................chaperon-mediated autophagy Cre...........................cyclase recombinase CSA..........................cross sectional area CTX..........................cardiotoxin DMEM..........................Dulbecco's modified eagle medium dmko...........................developmental muscle specific knock-out DNA..........................deoxyribonucleic acid EMSA........................ electrophoretic mobility shift assay eMyHC.......................embryonic isoform of myosin heavy chain ER............................ endoplasmic stress ERK.......................... extracellular signal-regulated kinase FoxO......................... forkhead box transcription factor 


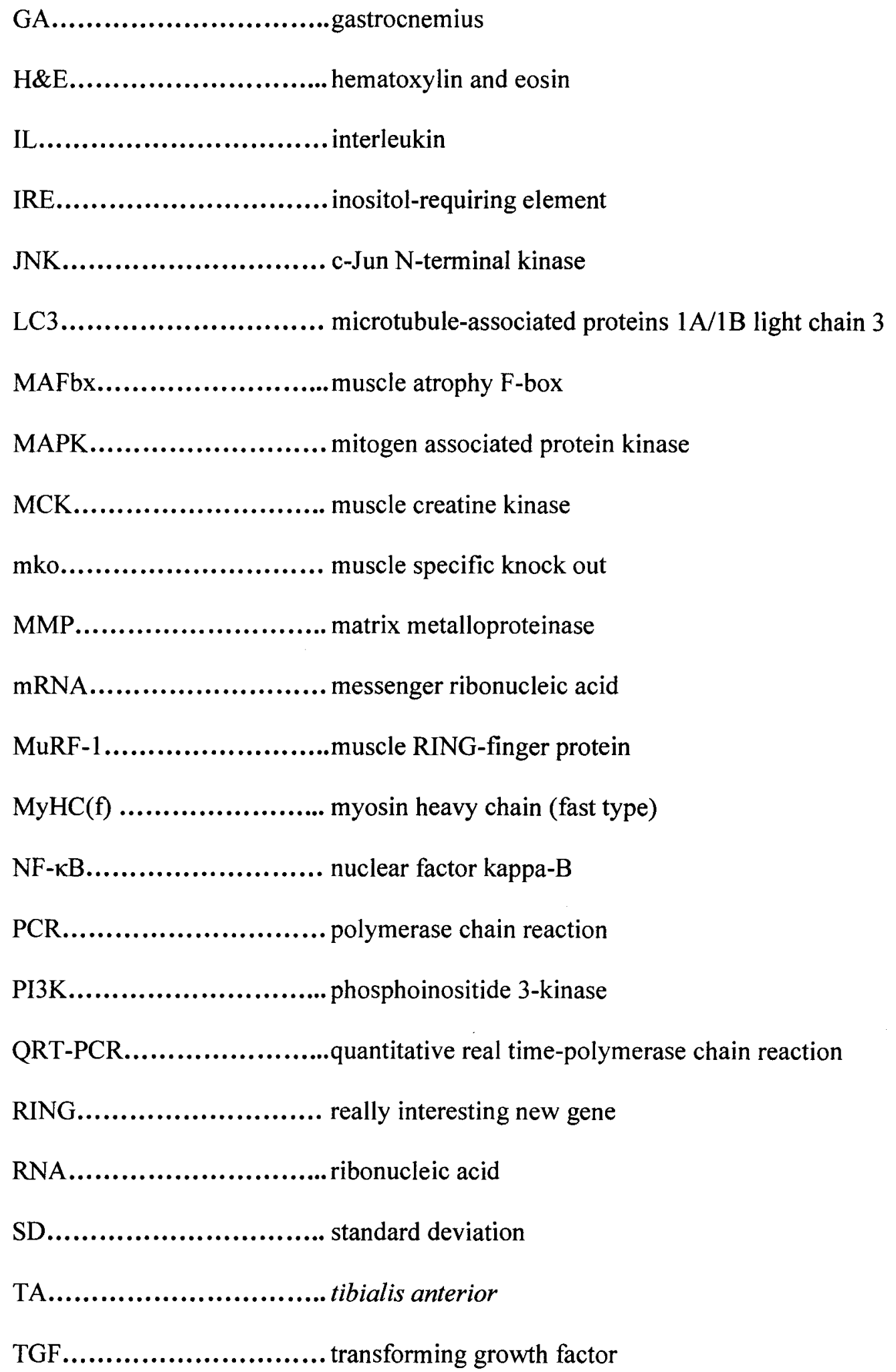


TLR........................... toll-like receptors

TNF........................... tumor necrosis factor

TNFR........................ tumor necrosis factor -receptor

TRAF......................... tumor necrosis factor -receptor associated factor 6

TWEAK........................ tumor necrosis factor-related weak inducer of apoptosis

Ub............................ ubiquitin

UPR............................unfolded protein response

UPS........................... ubiquitin-proteasome system

XBP1 ......................... X-box binding protein-1 


\section{CURRICULUM VITAE}

NAME:

ADRESS:
Pradyut K Paul

Department of Anatomical Sciences and Neurobiology School of Medicine, University of Louisville Louisville, KY 40202

EDUCATION \& TRAINING: Ph.D., Anatomical Sciences and Neurobiology School of Medicine, University of Louisville Louisville, KY

2007-2011

M.S., Biology

Western Kentucky University

Bowling Green, KY 42101

2006-2007

M.Sc., Biotechnology

AAI-DU, Allahabad, India 2001-2003

B.Sc., Biochemistry (Hons)

Gorakhpur University, India 1997-2000

PROFESSIONAL EXPERIENCE:

Graduate Research Assistant

Department of Anatomical Sciences and Neurobiology University of Louisville, Louisville, KY 40202 2007-2011

Graduate Research Assistant Department of Biology, Western Kentucky University Bowling Green, KY 42101 2006-2007 
Technical Coordinator

(Eppendorf Division)

Hysel India Limited

2005-2006

AWARDS \& HONORS:

Citation for excellence in research at Research

Louisville (annual research symposium and showcase in

Louisville) - 2010

Third prize in scientific poster session at Neuroscience Day 2010 (Society for Neuroscience, Louisville chapter)

Council for Scientific and Industrial Research scholarship for conducting research in any research Institution of India.

PROFESSIONAL SOCIETIES:

Member, American Association for the Advancement of Science $(2006$ - 2008, 2011 - 2013)

Member, American Society of Plant Biologists

(2006-2007)

Member, Society for Neuroscience $(2007-2011)$

PUBLICATIONS:

Paul PK, Kumar A (2012) TWEAK and TRAF6 mediate skeletal muscle atrophy. Current Opinion in Clinical Nutrition and Metabolic Care (review). To be published in May 2012 issue.

Paul PK, Dahiya S, Bhatnagar S, Choi Y and Kumar A (2011). Muscle specific deletion of TRAF6 improves repair and regeneration in skeletal muscles of mice. (manuscript under preparation).

Paul PK, Bhatnagar S, Dahiya S, Choi Y and Kumar A (2011). Toll-like receptor-TRAF6 signaling mediates starvation-induced skeletal muscle atrophy in mice (manuscript under editorial consideration in Molecular and Cellular Biology). 
Dahiya S, Bhatnagar S, Hindi S, Jiang J, Paul PK, Kuang $S$ and Kumar A (2011). Elevated levels of active matrix metalloproteinase-9 cause hypertrophy in skeletal muscle of normal and dystrophin-deficient mdx mice. Human Molecular Genetics (August 16, 2011)

Paul PK, Kumar A (2011). TRAF6 coordinates the activation of autophagy and ubiquitin-proteasome systems in atrophying skeletal muscle. Autophagy 1:7(5).

Paul PK, Gupta SK, Bhatnagar S, Panguluri SK, Darnay BG, Choi Y, Kumar A (2010). Targeted ablation of TRAF6 inhibits skeletal muscle wasting in mice. Journal of Cell Biology, 191: 1395-1411. (This article was also previewed as Editors' Choice in "Science Signaling, Vol. 4, Issue 154, p. ec3; 2011 " journal with heading "Understanding Atrophy")

Mittal A, Bhatnagar S, Kumar A, Paul PK, Kuang S, and Kumar A (2010). Genetic ablation of TWEAK augments regeneration and post-injury growth of skeletal muscle in mice. American Journal of Pathology, 177: 1732-1742. (An editorial was written for this article in the same issue of American Journal of Pathology)

Li H, Mittal A, Paul PK, Kumar M, Srivastava DS, Tyagi SC, Kumar A (2009). Tumor necrosis factor-related weak inducer of apoptosis augments matrix metalloproteinase 9 (MMP-9) production in skeletal muscle through the activation of nuclear factor-kappa B-inducing kinase and p38 mitogen-activated protein kinase: a potential role of MMP-9 in myopathy. J Biological Chemistry

284: 4439-50.

MEETING PRESENTATIONS:

Pradyut Paul, Sanjay Gupta, Shephali Bhatnagar, Siva Panguluri, Bryant Darnay, Yongwon Choi, Ashok Kumar (2010). Targeted Ablation of TRAF6 Prevents Atrophy and Promotes Skeletal Muscle Regeneration in Mice. Research Louisville, October 11 - 15, 2010. Louisville.

Pradyut Paul, Shephali Bhatnagar, Akhilesh Kumar, Sanjay Gupta, Ashok Kumar (2010). Specific Deletion of TNF-receptor associated factor 6 (TRAF6) Prevents 
Atrophy and Improves Skeletal Muscle Regeneration in Mice. 20th Annual Neuroscience Day. May 6, 2010. Louisville.

Pradyut Paul, Shephali Bhatnagar, Ashok Kumar. Skeletal muscle atrophy rescue in chronic diseases and disuse conditions by muscle-specific deletion of TRAF6. The Ottawa Conference on New Directions in Biology \& Disease of Skeletal Muscle May 5-8, 2010. Ottawa, Canada.

Pradyut K Paul, Nilesh C. Sharma, Shivendra V Sahi, Joseph Albano. Phosphatase activities and assimilation of organic phosphorus in L. multiflorum grown in- vitro. Plant Biology 2006 (ASPB): August 2006. Boston, Massachusetts.

Pradyut K Paul. Protoplast Culture Studies of Rosa spp. and Molecular Characterization of Panax quinquefolium L. Department of Biotechnology, Allahabad University, Allahabad, India. August 2003.

Pradyut K Paul. Molecular Authentication of Transgenic Plants by Southern Hybridization. Central Institute of Medicinal and Aromatic Plants (CSIR), Lucknow, India; June 2003.

Pradyut K Paul. Transformation Techniques and Recombinant DNA Technology. Department of Biochemistry, Allahabad Agricultural Institute, Allahabad; August 2002.

Pradyut K Paul. Protein Estimation and Enzyme Activity of Hydrolases and Reductases. Indian Institute of Sugarcane Research, Lucknow, India; July 2002. 